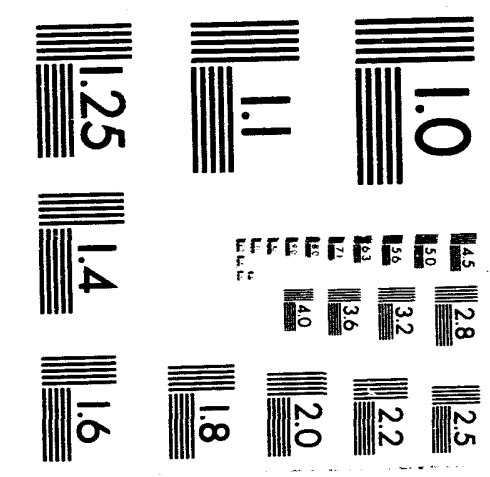



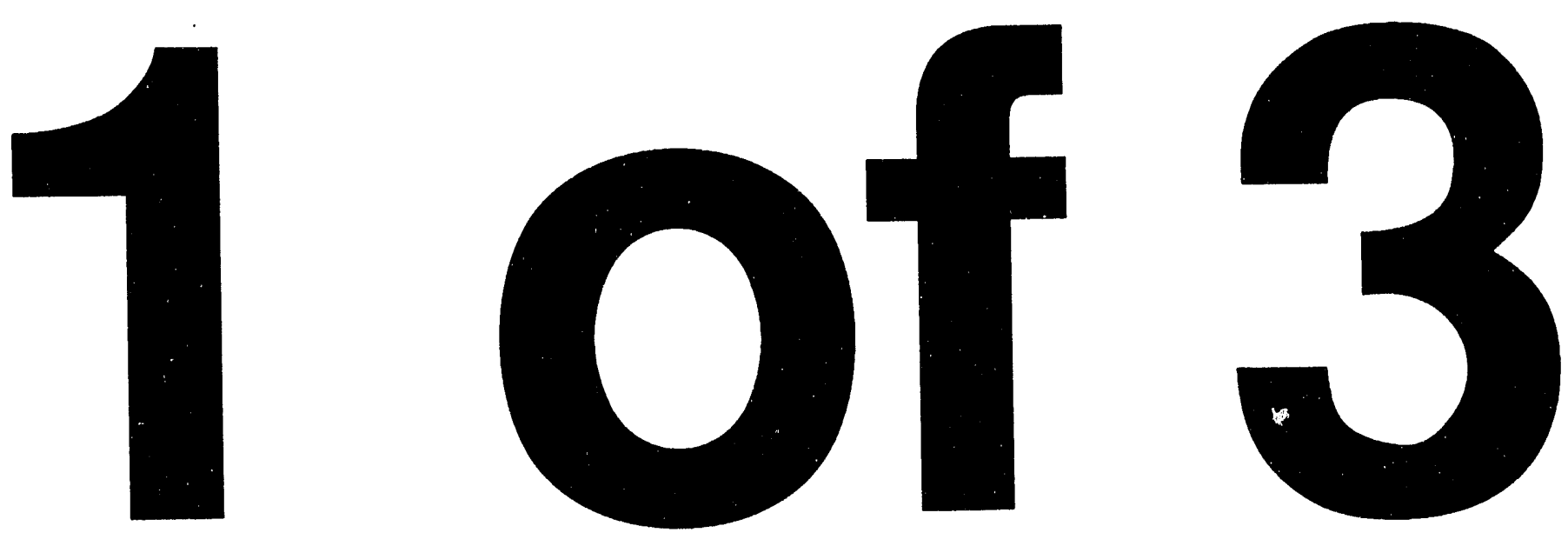


\title{
High-Temperature Superconducting Transformer Performance, Cost, and Market Evaluation
}

\author{
J. A. Dirks, Principal Investigator \\ J. E. Dagle \\ J. G. DeSteese \\ H. D. Huber \\ S. B. Merrick ${ }^{(a)}$ \\ S. A. Smith \\ T. A. Williams ${ }^{(\text {) }}$ \\ J. W. Currie, Project Manager
}

September 1993

Prepared for the U.S. Department of Energy under Contract DE-AC06-76RLO 1830

Pacific Northwest Laboratory

Richland, Washington 99352

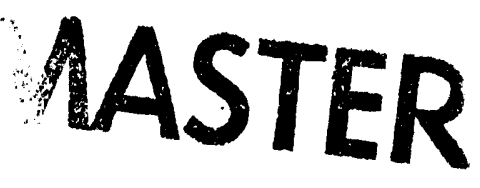

(a) Now with Westinghouse Hanford Company, Richland, Washington

(b) Now with the National Renewable Energy Laboratory, Golden, Colorado 


\section{Summary}

This report documents work performed by the Pacific Northwest Laboratory (PNL) for the U.S. Department of Energy (DOE) Office of Energy Storage and Distribution (OESD) to characterize the future performance, costs, market penetration, and national benefits of high-temperature superconducting (HTS) transformers. This : ffort was part of the OESD Superconducting Technology for Electric Power Systems (STEPS) Program. The overall objective of this PNL study was to evaluate the design feasibility and potential benefits of superconducting transformers built using advanced HTS materials.

A system of linked computer programs with teedbacks was developed for this purpose. This system performs a series of HTS transformer design and evaluation computations based on assumed future capabilities of HTS materials that may be achieved with further development. This approach also permits the conduct of sensitivity analysis to bound the impacts of important variables that are intrinsically uncertain, at present. For example, we were able to estimate the effect of filament diameter on HTS transformer performance and costs. These, in turn, affect sales potential and application benefits. Information of this type provides guidance on beneficial goals for future HTS materials development.

The system was designed with generic elements that are easily modified to analyze other HTS applications. It also provided the capability to investigate hypotheses that initially appear defensible, but when evaluated within a fully interdependent system, are shown to be less viable. For example, some experts believe there are overall system advantages from incorporating HTS materials with the highest possible critical current density $\left(\mathrm{J}_{\mathrm{o}}\right)$. Our results indicate that when optimizing on life-cycle cost, this is not necessarily true. We show that there is likely to be a $J_{0}$ "knee" at which the most optimal transformer design is found. Furthermore, our results indicate that for many designs, the optimal $\mathrm{J}_{\mathrm{c}}$ lies between $10^{4}$ and $10^{5} \mathrm{~A} / \mathrm{cm}^{2}$. This suggests that reaching $\mathrm{J}_{\mathrm{c}}$ of $10^{6} \mathrm{~A} / \mathrm{cm}^{2}$ and greater may not be necessary to realize significant benefits from the development and use of HTS transformers. We conclude that HTS research efforts should first target the development of materials with $\mathrm{J}_{\mathrm{c}}$ less than $10^{6} \mathrm{~A} / \mathrm{cm}^{2}$ for power transformer applications. Without the advantage of an interdependent modeling system, it would have been impossible to test the optimal $\mathrm{J}_{\mathrm{c}}$ hypothesis.

We also investigated the primary variables that determine the optimal $J_{c}$. We found the primary variable was the fields to which the conductors were exposed. This is a generic issue that impacts the competitiveness of all HTS electric power equipment, requires continued investigation, and will likely not be fully resolyed until the demonstration stage of this technology.

In this study, we evaluate two different types of HTS transformers and compare them to conventional and amorphous core transformers. The first HTS transformer type, a cryostable design, is the traditionai design that maintains operating stability through superconducting filaments embedded in a metal matrix. The second type, which we term "ultrastable," maintains operating stability through reduced operating current density and protection equipment. These two design approaches illustrate a range of performance; actual transformer designs are likely to be a combination of these two extremes. 
We found that HTS transformer designs, optimized on life-cycle cost, have higher efficiencies than their conventional counterparts at the same megavolt-ampere ratings. While ultrastable designs have the highest efficiency, cryostable designs are slightly more cost-effective primarily because the overall material requirements for cryostable designs are less than those for the ultrastable designs. On average, the life-cycle costs of optimized HTS transformer designs are approximately half the lifecycle costs of optimized conventional transformers.

We also investigated the potential for HTS transformers to be installed on electric utility systems as direct substitutions for conventional equipment. We concluded that the HTS transformer does not appear to present any major installation or operational problems. However, the need to operate and maintain refrigerant subsystems could present a major obstacle to utility acceptance of HTS transformers, even with the use of relatively inexpensive and widely accepted liquid nitrogen refrigeration schemes. We suggest that this potential problem can be overcome if utilities initiate a near-term program to gain operational experience with refrigerant subsystems.

The results of our study show that the nation will realize significant benefits from developing and using HTS transformers. These benefits will be realized through reductions in transformer lifecycle costs. Assuming that HTS sales begin in the year 2000 , we estimate benefits to be $\$ 15$ to $\$ 18$ billion through 2020 and $\$ 25$ to $\$ 28$ billion through 2030 . 


\section{Acknowledgments}

The authors acknowledge and thank Daryl Brown, Fred Johnson, and Bill Doggett for their many technical contributions to this project. Dr. Amit DasGupta, Project Manager for DOE's Office of Energy Storage and Distribution, Superconducting Technology for Electric Power Systems (OESDSTEPS) Research Program provided invaluable information, insight, and technical review that guided the direction and emphasis of the study.

Appreciation is also due to the members of the Study Advisory Group who contributed many hours during the course of the study in providing data, insights, and advice. The written comments of all reviewers are appreciated and reflected in this document. The in-depth review of the draft report provided by Dr. Susan Schoenung of W. J. Schafer Associates, Inc., is especially valued and noteworthy because it provided a comprehensive basis for improving the presentation and discussion of technical issues, thereby enhancing the report's overall technical quality and organization.

Finally, the authors acknowledge the technical communications expertise provided by editors/ writers Andrea J. Currie and Cathy A. Anderson in editing and assembling the final manuscript. 


\section{Contents}

Summary $\quad \ldots \ldots \ldots \ldots \ldots \ldots \ldots \ldots \ldots \ldots \ldots \ldots \ldots \ldots \ldots \ldots \ldots$ iii

Acknowledgments $\ldots \ldots \ldots \ldots \ldots \ldots \ldots \ldots \ldots \ldots \ldots \ldots \ldots \ldots$

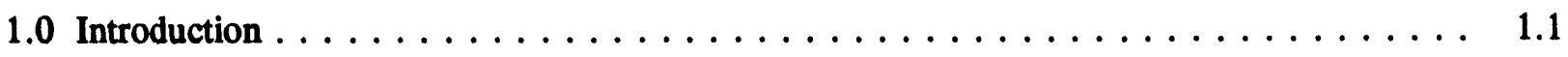

1.1 Approach $\ldots \ldots \ldots \ldots \ldots \ldots \ldots \ldots \ldots \ldots \ldots \ldots \ldots \ldots \ldots \ldots \ldots \ldots$

1.2 Report Organization and Scope $\ldots \ldots \ldots \ldots \ldots \ldots \ldots \ldots \ldots \ldots$

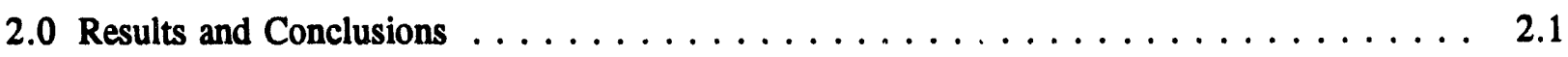

2.1 Transformer Design/Performance $\ldots \ldots \ldots \ldots \ldots \ldots \ldots \ldots \ldots \ldots \ldots \ldots$

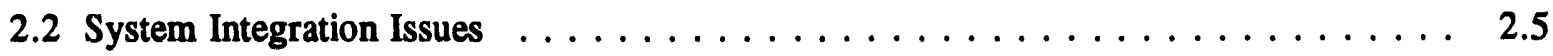

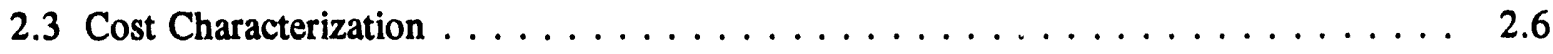

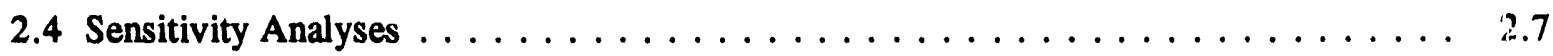

2.5 Market Penetration/Benefits to the Nation $\ldots \ldots \ldots \ldots \ldots \ldots \ldots$

3.0 Design and Performance Characterization $\ldots \ldots \ldots \ldots \ldots \ldots \ldots \ldots \ldots$

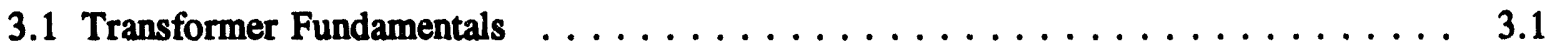

3.1.1 Ideal Transformer Theory $\ldots \ldots \ldots \ldots \ldots \ldots \ldots \ldots \ldots \ldots \ldots \ldots \ldots$

3.1.2 Issues Related to Superconductive Transformer Design $\ldots \ldots \ldots \ldots \ldots \ldots$. . . 3.3

3.2 Major Transformer Loss Mechanisms - Load Losses and No-Load Losses . . . . . . 3.4

3.2.1 Transformer Load Losses $\ldots \ldots \ldots \ldots \ldots \ldots \ldots \ldots \ldots \ldots$

3.2.2 Transformer No-Load Losses $\ldots \ldots \ldots \ldots \ldots \ldots \ldots \ldots \ldots \ldots$

3.2.3 Superconductive Transformer Cooling . . . . . . . . . . . . 3.12

3.2.4 Major Transformer Loss Mechanisms . . . . . . . . . . . . . . . . . . 3.12

3.3 Transformer Design Principles and Practices $\ldots \ldots \ldots \ldots \ldots \ldots \ldots$

3.3.1 Superconductive Transformer Design $\ldots \ldots \ldots \ldots \ldots \ldots \ldots . \ldots \ldots$

3.4 Key Equations and Relationships $\ldots \ldots \ldots \ldots \ldots \ldots \ldots \ldots \ldots \ldots$ 
3.4.1 Core Expressions - Core Loss, Leakage Field, and Stray Loss Factor . . . . 3:16

3.4 .2 Self-Field $\ldots \ldots \ldots \ldots \ldots \ldots \ldots \ldots \ldots \ldots \ldots \ldots \ldots \ldots \ldots$

3.4.3 Superconductor Cryostability Design Methodology $\ldots \ldots \ldots \ldots \ldots \ldots \ldots$

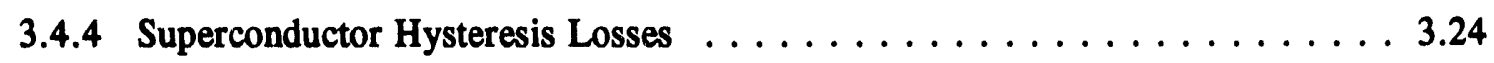

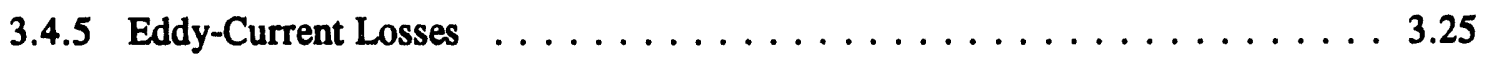

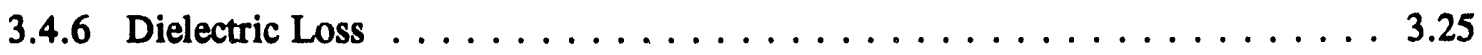

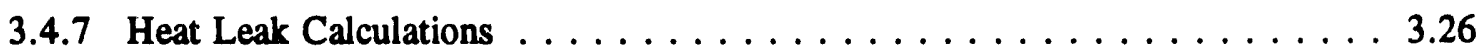

3.4.8 Refrigeration System Coefficient of Performance $\ldots \ldots \ldots \ldots \ldots \ldots$

3.5 Base Case Design Parameters $\ldots \ldots \ldots \ldots \ldots \ldots \ldots \ldots \ldots \ldots \ldots$

3.5.1 Base Case Design Selections . . . . . . . . . . . . . 3.28

3.5.2 Base Case Superconductive Material Specifications . . . . . . . . . 3.30

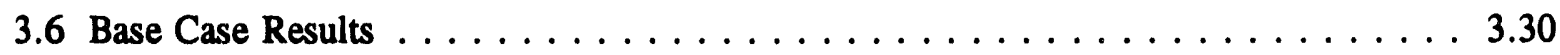

3.6.1 Base Case Conventional Transformer Design and Performance Results . . . 3.32

3.6.2 Base Case Cryostable Transformer Design and Performance Results . . . . . 3.35

3.6.3 Base Case Ultrastable Transformer Design and Performance Results . . . . . 3.44

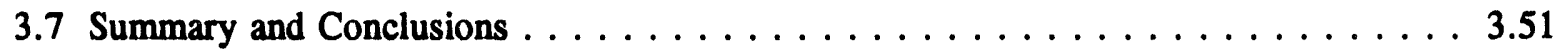

4.0 System Integration Considerations $\ldots \ldots \ldots \ldots \ldots \ldots \ldots \ldots \ldots \ldots \ldots$

4.1 Basic Assumptions $\ldots \ldots \ldots \ldots \ldots \ldots \ldots \ldots \ldots \ldots \ldots \ldots \ldots \ldots$

4.2 Advantages and Benefits of HTS Transformers . . . . . . . . . . . . 4.1

4.3 System Integration Issues Considered $\ldots \ldots \ldots \ldots \ldots \ldots \ldots \ldots \ldots \ldots$

4.3.1 Utility Acceptance of the Refrigeration Subsystem . . . . . . . . . . . . 4.3

4.3.2 Protection of the Cryogenic Subsystem . . . . . . . . . . . . . . 4.4

4.3.3 Protection Against System Faults and Overloads . . . . . . . . . . . . . 4.5

4.3.4 Operation and Maintenance $\ldots \ldots \ldots \ldots \ldots \ldots \ldots \ldots \ldots$ 
4.3.5 Environmental Issues $\ldots \ldots \ldots \ldots \ldots \ldots \ldots \ldots \ldots \ldots \ldots \ldots .4 .7$

4.4 Summary Comments $\ldots \ldots \ldots \ldots \ldots \ldots \ldots \ldots \ldots \ldots \ldots .8$

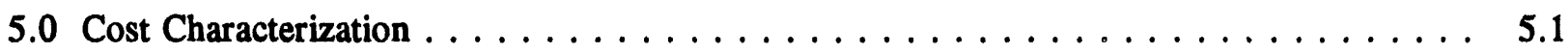

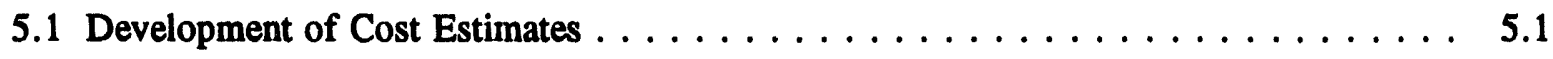

5.2 Approach Used $\ldots \ldots \ldots \ldots \ldots \ldots \ldots \ldots \ldots \ldots \ldots \ldots \ldots .2$

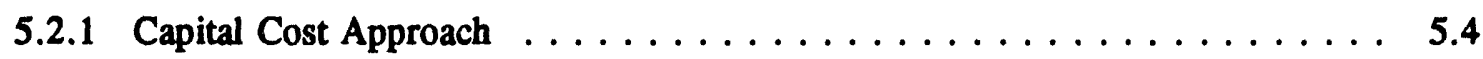

5.2 .2 Costs and Price Changes $\ldots \ldots \ldots \ldots \ldots \ldots \ldots \ldots \ldots \ldots .8 \ldots \ldots \ldots \ldots$

5.2.3 Cost Estimates for Operation and Maintenance $\ldots \ldots \ldots \ldots \ldots \ldots .13$

5.2 .4 Value of Energy Losses $\ldots \ldots \ldots \ldots \ldots \ldots \ldots \ldots \ldots \ldots .13$

5.2.5 Calculations of Life-Cycle Costs $\ldots \ldots \ldots \ldots \ldots \ldots \ldots \ldots \ldots .15$

5.3 Results $\ldots \ldots \ldots \ldots \ldots \ldots \ldots \ldots \ldots \ldots \ldots \ldots \ldots \ldots \ldots .17$

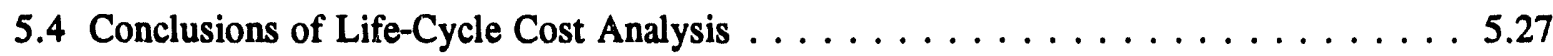

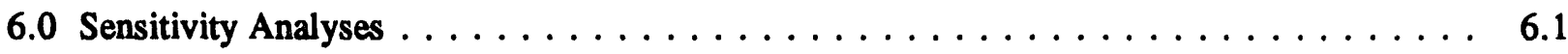

6.1 Influence of Critical Current Density on Transformer Performance, Design,

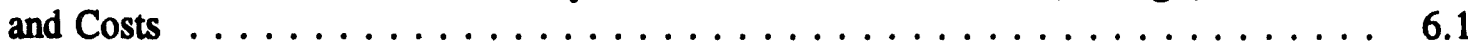

6.1.1 Sensitivity Analysis with $\mathrm{J}_{\mathrm{c}}$ for Cryostable Transformer Performance

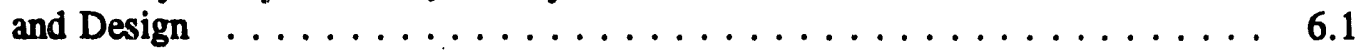

6.1.2 Life-Cycle Cost Impacts for the Cryostable Transformer Designs . . . . . . 6.5

6.1.3 Summary of $J_{\mathrm{c}}$ Sensitivity Analysis for Cryostable Designs $\ldots \ldots \ldots \ldots .6$

6.1.4 Sensitivity Analysis with $\mathrm{J}_{\mathrm{c}}$ for Ultrastable Transformer Designs $\ldots \ldots \ldots 6.8$

6.1.5 Life-Cycle Cost Impacts for the Ultrastable Transformer Designs . . . . . . . 6.11

6.1.6 Summary of $\mathrm{J}_{\mathrm{c}}$ Sensitivity Analysis for Ultrastable Designs . . . . . . . 6.11

6.1.7 Critical Current Density Sensitivity Analysis Conclusions $\ldots \ldots \ldots \ldots \ldots .12$

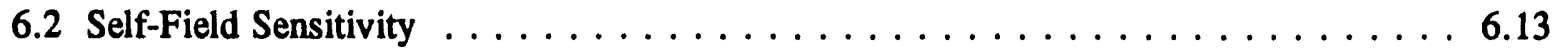

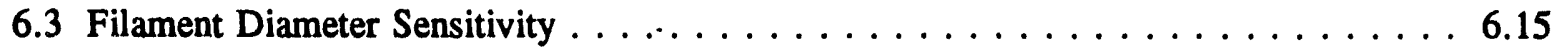




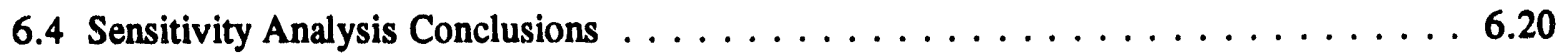

7.0 Market Penetration and Benefits to the Nation $\ldots \ldots \ldots \ldots \ldots \ldots \ldots$

7.1 Development of Initial Transformer Inventory $\ldots \ldots \ldots \ldots \ldots \ldots$

7.1.1 Annual Generating Capacity Changes $\ldots \ldots \ldots \ldots \ldots \ldots \ldots$

7.1.2 Annual Generator Installations $\ldots \ldots \ldots \ldots \ldots \ldots \ldots \ldots \ldots$

7.1.3 Total Transformer Installations Each Year $\ldots \ldots \ldots \ldots \ldots \ldots$

7.1.4 Number of Transformers Installod Each Year by Type $\ldots \ldots \ldots \ldots \ldots$

7.1.5 Calculation of Surviving Transformers by Year $\ldots \ldots \ldots \ldots \ldots$

7.2 Utility Market Penetration of HTS $\ldots \ldots \ldots \ldots \ldots \ldots \ldots$

7.2.1 Overview of the Transformer Selection Process $\ldots \ldots \ldots \ldots \ldots \ldots$

7.2.2 Transformer Selection Process Details . . . . . . . . . . . . . 7.10

7.2.3 Valuing Energy Losses $\ldots \ldots \ldots \ldots \ldots \ldots \ldots \ldots \ldots \ldots$

7.2.4 Selecting HTS Versus Conventional Transformers $\ldots \ldots \ldots \ldots \ldots \ldots . . \ldots 73$

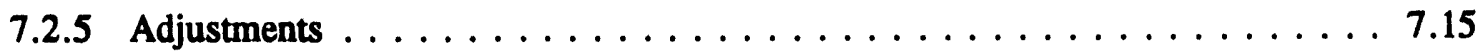

7.3 Benefits to the Nation $\ldots \ldots \ldots \ldots \ldots \ldots \ldots \ldots \ldots \ldots \ldots \ldots$

7.4 Market Penetration Evaluation of HTS Transformer Designs $\ldots \ldots \ldots \ldots \ldots \ldots$

7.4.1 Dollar Savings from Adopting HTS Transformers $\ldots \ldots \ldots \ldots \ldots . \ldots 78$

7.4.2 Energy Savings from Adopting HTS Transformers . . . . . . . . . . . . 7.19

7.4.3 Market Penetration Rate of HTS Transformers $\ldots \ldots \ldots \ldots . . \ldots \ldots . . \ldots 722$

8.0 References $\ldots \ldots \ldots \ldots \ldots \ldots \ldots \ldots \ldots \ldots \ldots \ldots \ldots \ldots . \ldots \ldots . . \ldots \ldots$

Appendix A - Study Advisory Group $\ldots \ldots \ldots \ldots \ldots \ldots \ldots \ldots \ldots \ldots \ldots \ldots$

Appendix B - Transformer Design Model Formulation $\ldots \ldots \ldots \ldots \ldots \ldots$

Appendix C - EAST Code Description $\ldots \ldots \ldots \ldots \ldots \ldots \ldots \ldots \ldots \ldots \ldots \ldots \ldots \ldots$

Appendix D - HTS Market Assessment Program $\ldots \ldots \ldots \ldots \ldots \ldots \ldots \ldots$ 


\section{Figures}

2.1 Efficiency of Grid Transformers Designed for Lowest Life-Cycle Cost $\ldots \ldots \ldots$. . . . . 2.3

2.2 Life-Cycle Cost Comparison, 1000-MVA Grid, Si-Steel Core $\ldots \ldots \ldots \ldots$

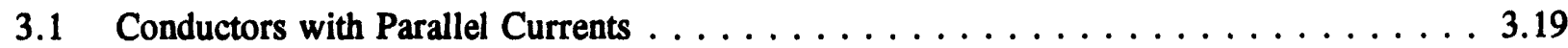

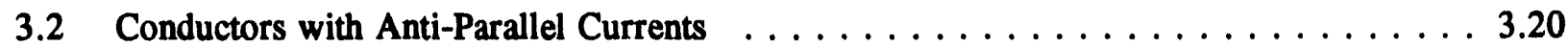

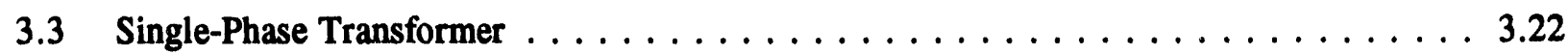

5.1 Capital Cost Adjustment Factors for HTS Transformers in Early Production Years . . . 5.12

5.2 Example of Transformer Design Evaluation $\ldots \ldots \ldots \ldots \ldots \ldots \ldots$

5.3 Comparison of EAST Model Test Results to Purchase Cost Data $\ldots \ldots \ldots \ldots . \ldots$

5.4 Life-Cycle Cost Comparison for Grid Transformers with Si-Steel Core . . . . . . . . . 5.19

5.5 Life-Cycle Cost Breakdown for 30-MVA Grid Transformers with Si-Steel Core . . . . 5.19

5.6 Life-Cycle Cost Breakdown for 200-MVA Grid Transformers with Si-Steel Core . . . 5.20

5.7 Life-Cycle Cost Breakdown for 1000-MVA Grid Transformers with Si-Steel Core . . . . 5.20

5.8 Life-Cycle Cost Comparison for Step-Up Transformers with Si-Steel Core $\ldots \ldots$. . . 5.21

5.9 Life-Cycle Cost Breakdown for 60-MVA Step-Up Transformers with Si-Steel Core . . . 5.22

5.10 Life-Cycle Cost Breakdown for 310-MVA Step-Up Transformers with Si-Steel Core . . 5.22

5.11 Life-Cycle Cost Breakdown for 630-MVA Step-Up Transformers with Si-Steel Core . . 5.23

5.12 Comparison of Amorphous and Si-Steel Core Life-Cycle Costs for HTS-CS and

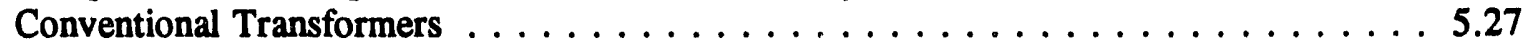

6.1 Cryostable Transformer Individual Power Losses Versus Critical Current

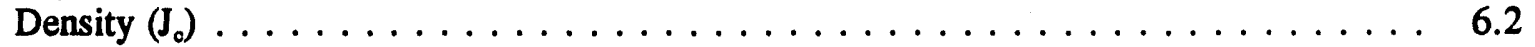

6.2 Transformer Efficiency as a Function of $\mathrm{J}_{\mathrm{c}} \ldots \ldots \ldots \ldots \ldots$

6.3 Transformer Life-Cycle Cost as a Function of $J_{c} \ldots \ldots \ldots \ldots$

6.4 Impact of Various $\mathrm{J}_{\mathrm{c}}$ Assumptions on Transformer Life-Cycle Costs $\ldots \ldots \ldots .7$

6.5 Impact of $\mathrm{J}_{\mathrm{o}}$ on HTS-Cryostable Life-Cycle Capital and Energy Cost $\ldots \ldots \ldots \ldots$ 
6.6 Ultrastable Transformer Individual Power Losses Versus Critical Current

Density $\left(J_{c}\right) \ldots \ldots \ldots \ldots \ldots \ldots \ldots \ldots \ldots \ldots \ldots \ldots \ldots$

6.7 Impact of $J_{c}$ on HTS-Ultrastable Life-Cycle Capital and Energy Cost $\ldots \ldots \ldots . \ldots$

5.8 Effect of Superconductive Filament Diameter on Life-Cycle Costs . . . . . . . . . . 6.19

7.1 Methodology for Estimating Market Penetration and Benefits to the Nation $\ldots \ldots \ldots .2$

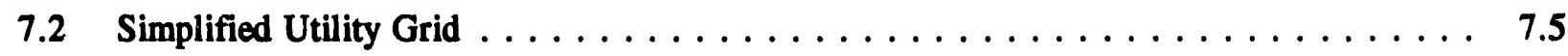

7.3 Transformer Annual Replacement Probability $\ldots \ldots \ldots \ldots \ldots \ldots \ldots$

7.4 Transformer Selection Methodology $\ldots \ldots \ldots \ldots \ldots \ldots \ldots$

7.5 The Averages of Energy Values Greater than $\mathrm{P}^{*}, \ldots \ldots \ldots \ldots \ldots \ldots$

7.6 Dollar Benefits from Adopting HTS $\ldots \ldots \ldots \ldots \ldots \ldots \ldots \ldots \ldots \ldots \ldots$

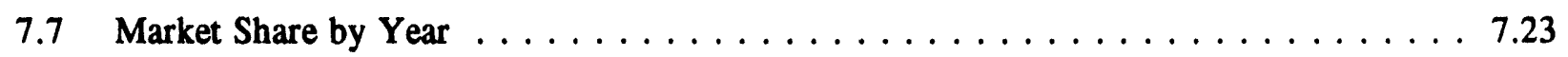




\section{Tables}

2.1 Base Case Superconductor Material Parameters $\ldots \ldots \ldots \ldots \ldots \ldots \ldots$

2.2 Conventional and Superconducting Transformer Losses $\ldots \ldots \ldots \ldots \ldots$

3.1 Transformer Base Case Design Selections . . . . . . . . . . . . . . . . 3.29

3.2 Base Case Material Specifications $\ldots \ldots \ldots \ldots \ldots \ldots \ldots \ldots \ldots \ldots \ldots \ldots$

3.3 Conventional and Superconducting Transformer Losses $\ldots \ldots \ldots \ldots \ldots \ldots$

3.4 Conventional Grid Transformer Performance; Conventional Core . . . . . . . . . 3.33

3.5 Conventional Grid Transformer Performance; Amorphous Core . . . . . . . . . . 3.34

3.6 Conventional Step-Up Transformer Performance; Conventional Core $\ldots \ldots \ldots \ldots$

3.7 Conventional Step-Up Transformer Performance; Amorphous Core . . . . . . . . . . 3.37

3.8 Conventional Transformer Design Results $\ldots \ldots \ldots \ldots \ldots \ldots \ldots \ldots$

3.9 Cryostable Grid Transformer Performance; Conventional Core . . . . . . . . . . . . 3.39

3.10 Cryostable Step-Up Transformer Performance; Conventional Core . . . . . . . . . . . 3.40

3.11 Cryostable Grid Transformer Performance; Amorphous Core . . . . . . . . . . 3.41

3.12 Cryostable Step-Up Transformer Performance; Amorphous Core . . . . . . . . 3.42

3.13 Cryostable Transformer Design Results $\ldots \ldots \ldots \ldots \ldots \ldots \ldots \ldots \ldots$

3.14 Ultrastable Grid Transformer Performance; Conventional Core . . . . . . . . . . . . . 3.45

3.15 Ultrastable Step-Up Transformer Performance; Conventional Core . . . . . . . . . 3.46

3.16 Ultrastable Grid Transformer Performance; Amorphous Core . . . . . . . . . . . . 3.48

3.17 Ultrastable Step-Up Transformer Performance; Amorphous Core . . . . . . . . . . . 3.49

3.18 Ultrastable Transformer Design Results $\ldots \ldots \ldots \ldots \ldots \ldots$

5.1 Transfer Cost Elements $\ldots \ldots \ldots \ldots \ldots \ldots \ldots \ldots \ldots \ldots \ldots \ldots$

5.2 Adjustment Factors for HTS Materials $\ldots \ldots \ldots \ldots \ldots \ldots \ldots \ldots \ldots \ldots \ldots$

5.3 Adjustment Factors for Transformer Shell, Cryocooler, and Risk Premium . . . . . . 5.12 


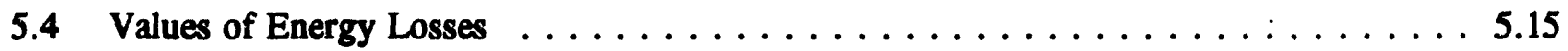

5.5 General Economic Assumptions $\ldots \ldots \ldots \ldots \ldots \ldots \ldots \ldots \ldots$

5.6 Capital Cost Breakdown for Grid Transformers with Si-Steel Core . . . . . . . . . . 5.25

5.7 Capital Cost Breakdown for Step-Up Transformers with Si-Steel Core $\ldots \ldots$. . . . . . 5.25

5.8 Annual O\&M Cost Results for Grid Transformers $\ldots \ldots \ldots \ldots \ldots \ldots$

5.9 Annual O\&M Cost Results for Step-Up Transformers $\ldots \ldots \ldots \ldots \ldots \ldots$

6.1 Key Desigii Resilts for 1000-MVA Cryostable Autotransformers as a Function of $J_{c} \quad \ldots \quad 6.3$

6.2 Key Design Results for 1000-MVA Ultrastable Autotransformers as a Function of $J_{c} \quad \ldots \quad 6.9$

6.3 Key Design Results for Self-Field Sensitivity $\ldots \ldots \ldots \ldots \ldots \ldots \ldots$

6.4 Performance Results for Self-Field Sensitivity-Cryostable--1000-MVA

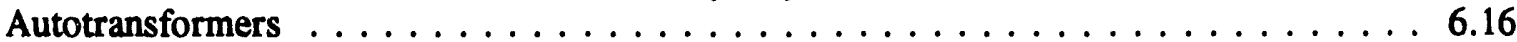

6.5 Performance Results for Self-Field Sensitivity-Ultrastable--1000-MVA

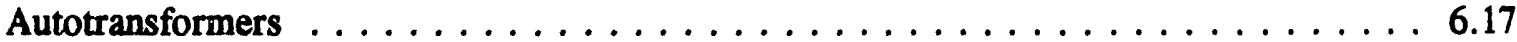

6.6 Life-Cycle Cost Results for Self-Field Sensitivity Analysis . . . . . . . . . . . . . . 6.18

6.7 Full-Load Loss Results for Filament Diameter Sensitivity Analysis . . . . . . . . . . . 6.19

7.1 Net Summer Generation Capability for Electric Utilities $\ldots \ldots \ldots \ldots \ldots \ldots$

7.2 Transformer Capacity Installed (MVA) per MW of Generator Installation $\ldots \ldots \ldots$

7.3 Dollar Benefits from Adopting HTS Transformers, Base Case $\ldots \ldots \ldots \ldots$. . . . . 7.20

7.4 Dollar Benefits from Adopting HTS Transformers, Sensitivity Case $\ldots \ldots \ldots$. . . . . 7.20

7.5 Energy Savings from HTS Transformers $\ldots \ldots \ldots \ldots \ldots \ldots \ldots \ldots \ldots \ldots \ldots$

7.6 Value of Market Penetration Adjustment Factors for 1000-MVA Conventional Core Cryostable Transformers $\ldots \ldots \ldots \ldots \ldots \ldots \ldots \ldots \ldots \ldots \ldots \ldots . \ldots \ldots \ldots$ 


\subsection{Introduction}

Recent laboratory breakthroughs in high-temperature superconducting (HTS) materials have stimulated both the scientific community and general public with questions regarding how these materials can be used in practical applications. While there are obvious benefits from using HTS materials (most notably the potential for reduced energy losses in the conductors), a number of issues (such as overall system energy losses, cost, and reliability) may limit applications of HTS equipment, even if the well known materials problems are solved. This study examined the future application potential of HTS materials to power transformers.

This study effort was part of a U.S. Department of Energy (DOE) Office of Energy Storage and Distribution (OESD) research program, Superconductivity Technology for Electric Power Systems (STEPS). The study took a systems perspective to gain insights to help guide DOE in managing research designed to realize the vision of HTS applications. Specific objectives of the study were as follows:

- to develop an understanding of the fundamental HTS transformer design issues that can provide guidance for developing practical devices of interest to the electric utility industry

- to identify electric utility requirements for HTS transformers and to evaluate the potential for developing a commercial market

- to evaluate the market potential and national benefits for HTS transformers that could be achieved by a successful HTS development program

- to develop an integrated systems analysis framework, which can be used to support R\&D planning by DOE, by identifying how various HTS materials characteristics impact the performance, cost, and national benefits of the HTS application.

\subsection{Approach}

Understanding and describing the market potential for electrical equipment that has not yet been developed are formidable tasks. A defensible analysis requires a methodology that appropriately models the performance, cost, and market factors. The methodology must also allow for sensitivity analyses to bound the impact of important variables that are intrinsically uncertain. Our approach accomplishes this. Furthermore, the methods and tools we developed not only accomplish these objectives for HTS transformers, but they are also generic enough to be easily modified for analysis of other HTS applications.

Our approach to the evaluation of HTS equipment considers four interrelated activities: systems integration, system performance, system cost, and market penetration. Systems integration, performance, and cost all are criteria that influence the design of the HTS application. Systems integration issues include how the HTS equipment will interact with other equipment in the utility grid, as well as design features that must be buiit into the HTS application to obtain utility 
acceptance. System performance deals with the energy consumption (efficiency) of the HTS application and how this is affected by alternative design approaches and materials characteristics. System cost deals with the initial and operating costs of the application and evaluates how life-cycle costs are affected by alternative designs; minimizing the life-cycle cost provides the basis for selecting an optimal design. Finally, the market penetration task addresses the extent to which the utility industry is likely to adopt the HTS equipment based on its lite-cycle cost, capital cost, performance, and perceived risk.

Evaluating the potential of HTS transformers requires comparing an HTS design to an equivalent service conventional transformer design. To ensure that our results reflected an equitable comparison, we applied the same basic design, cost, and market penetration approach to the conventional transformers as was used for HTS transformers, rather than simply gather data on existing conventional transformers. Such methodological consistency in the evaluation is imperative because different application assumptions (such as the value of energy losses) can significantly impact the final transformer design. Our study investigated two common transformer types (grid transformers and step-up transformers) at a wide range of sizes to identify any particular niche markets that HTS transformers might occupy. We also investigated a relatively new transformer design trend, using amorphous core materials, to determine whether further advances in this area would tend to impact the commercial viability of HTS transformers.

The need for evaluating the impact of a large number of designs, applications, and material parameters led us to developing a family of computer codes that can design an HTS (or conventional) transformer, estimate its performance, optimize the design on the basis of life-cycle cost, and then estimate the annual sales of the transformer. As indicated earlier, the codes have been designed to be as generic as possible to allow the analysis of other HTS applications with minimal code revisions. The performance, economics, and market codes can be operated independently if desired; operated sequentially, these codes provide an integrated picture of how the many variables come together to affect the ultimate value of an HTS transformer. The impact of the HTS filament diameter on the design of an optimized transformer is an example of the type of investigation possible with the models. A change in filament diameter alters the transformer energy losses, which results in a new optimal transformer design with a different life-cycle cost and a new estimate of yearly transformer sales. A wide range of investigations of this type is possible with the models, allowing insights into the interaction among materials characteristics, design parameters, cost, and market penetration.

Because the focus of this effort was to identify and evaluate technology that will be accepted by the utility industry, it was important for us to have access to information from the industry that would ultimately supply the HTS equipment and the electric utility industry. To accomplish this, we formed a Study Advisory Group (SAG) comprising experts from various industrial companies and utilities. Members of the SAG made invaluable contributions at various stages of the research, including reviewing interim results, suggesting analytical approaches, and providing key data. A listing of the SAG is provided in Appendix A. 


\subsection{Report Organization and Scope}

This report provides a detailed description of the study approach and results. It is organized into seven chapters.

Chapter 2 summarizes and explains our conclusions and recommendations. This is a "stand-alone" chapter with a description of underlying assumptions and base case parameters.

Transformer design and performance are covered in Chapter 3. The $r$-eatest share of project resources was devoted to research in support of this chapter, and its lengtl. and comprehensiveness reflect this. We begin with the basics of the "ideal" transformer and work through the development of a model capable of designing conventional and HTS transformers. We then use the model to design, evaluate, and compare different conventional and HTS transformer types.

Systems integration issues are discussed in Chapter 4 . We identify potential barriers to the use of HTS transformers within the nation's existing and future electric power system. Where appropriate, we either resolve issues or identify the steps necessary for resolution.

Cost characterization is the subject of Chapter 5. We combine several cost models into a general methodology for estimating the life-cycle costs of HTS equipment. The methodology is then linked with the transformer performance and design model to produce optimal designs and costs for different conventional and HTS transformer types.

Sensitivity analyses are presented in Chapter 6 . The sensitivity cases that we evaluate are of three different types: the transformer performance and design model, the cost model, and the linked transformer model and cost model system that produces information on optimal transformer designs.

The market and benefits analyses are reported in Chapter 7. We develop and present market and benefit models that are generic to electric power system equipment. Using optimal HTS transformer designs, we then project the market for the equipment and estimate the cost and energy savings to the nation from the availability of HTS transformers. 


\subsection{Results and Conclusions}

In this chapter, we present a brief overview of the major results and conclusions from our study. These conclusions are valid for the transformers identified as optimal designs from a life-cycle cost standpoint in our study, and, as such, do not pertain to questions regarding maximum theoretical efficiency. In addition, these conclusions are valid only for the base case material parameters.

More detailed discussions of the results and conclusions are found in subsequent chapters. Changes in the materials parameters are considered in Chapter 6, Sensitivity Analyses.

\subsection{Transformer Design/Performance}

In our study, two types of HTS transformers are compared with conventional transformer technology. The first type, a cryostable design, is the traditional design approach for superconducting equipment; it achieves operating stability through superconducting filaments embedded in a metal matrix called the stabilizer. These form strands bundled into conductors. The second type represents a nontraditional design approach, where superconductor operating stability is achieved by reducing the operating current density (and by using additional protection equipment), rather than by using stability metal. We call this type of transformer an "ultrastable" design. In our analysis, we have investigated an ultrastable design criterion where the maximum normal operating current density $(\mathrm{J})$ was fixed at between $5 \%$ and $7 \%$ of critical current density $\left(\mathrm{J}_{c}\right)$. Thus, the ultrastable designs are capable of operating with transients of 14 to 20 times the rated operating current. In actual practice, a combination of these design approaches would be used. However, analyzing these two design approach extremes brackets the range of performance results.

The major drivers of the design and performance results are the superconductor material parameters. The base case values for the most important parameters are presented in Table 2.1. The stated $J_{c}$ value assumes that the conductor will remain superconductive at or near the temperature of liquid nitrogen in the presence of the magnetic fields normally encountered in a transformer.

Table 2.1. Base Case Superconductor Material Parameters

\begin{tabular}{lc}
\multicolumn{1}{c}{ HTS Material Parameter } & Base Case Value \\
\hline Critical current density $\left(J_{c}\right)$ & $10^{5} \mathrm{~A} / \mathrm{cm}^{2(a)}$ \\
Strand radius ( $\left.r_{a}\right)$ & $500 \mu \mathrm{m}$ \\
Filament diameter (D) & $50 \mu \mathrm{m}$ \\
Twist pitch $\left(L_{p}\right)$ & $2 \mathrm{~cm}$
\end{tabular}

(a) At $77 \mathrm{~K}$ and maximum magnetic field intensity in transformer windings. 
Table 2.2 shows the types of losses that we modeled 1 the design and performance analysis. (Refer to Sections 3.2 and 3.4 for discussions of the losses and equations.) The cooling system of the superconducting transformer requires input of electrical energy to remove heat generated by losses within the cryostat and heat entering the cryostat from the environment. Heat that leaks into the cryostat through the vacuum vessel walls and through the conductor terminal bushings is not an electrical loss but does represent an additional load on the cooling system. This effectively becomes an additional loss term for the superconducting transformer.

Of the losses listed in Table 2.2, those that must be removed by the cryocooling system have a disproportionate effect on the transformer efficiency. These losses are hysteresis losses in the superconductor, eddy-current losses in the stabilizer, dielectric losses in the insulation material, and heat leak into the cryostat; all are scaled by the coefficient of performance (COP) of the cooling system. At liquid nitrogen temperature, the losses are 10 to 20 times more costly than they would be at ambient temperature.

One of our first investigations was the criterion that should be used to guide the transformer design. One approach was to design the HTS transformer to achieve a maximum reasonable efficiency. The alternative considered was to have feedback from the life-cycle cost and to optimize (within the constraints of the design assumptions) the transformer design to achieve the lowest possible life-cycle cost. In comparing the approaches, we found that the transformers designed for the best efficiency were always much less cost-effective than transformers designed based on the

Table 2.2. Conventional and Superconducting Transformer Losses

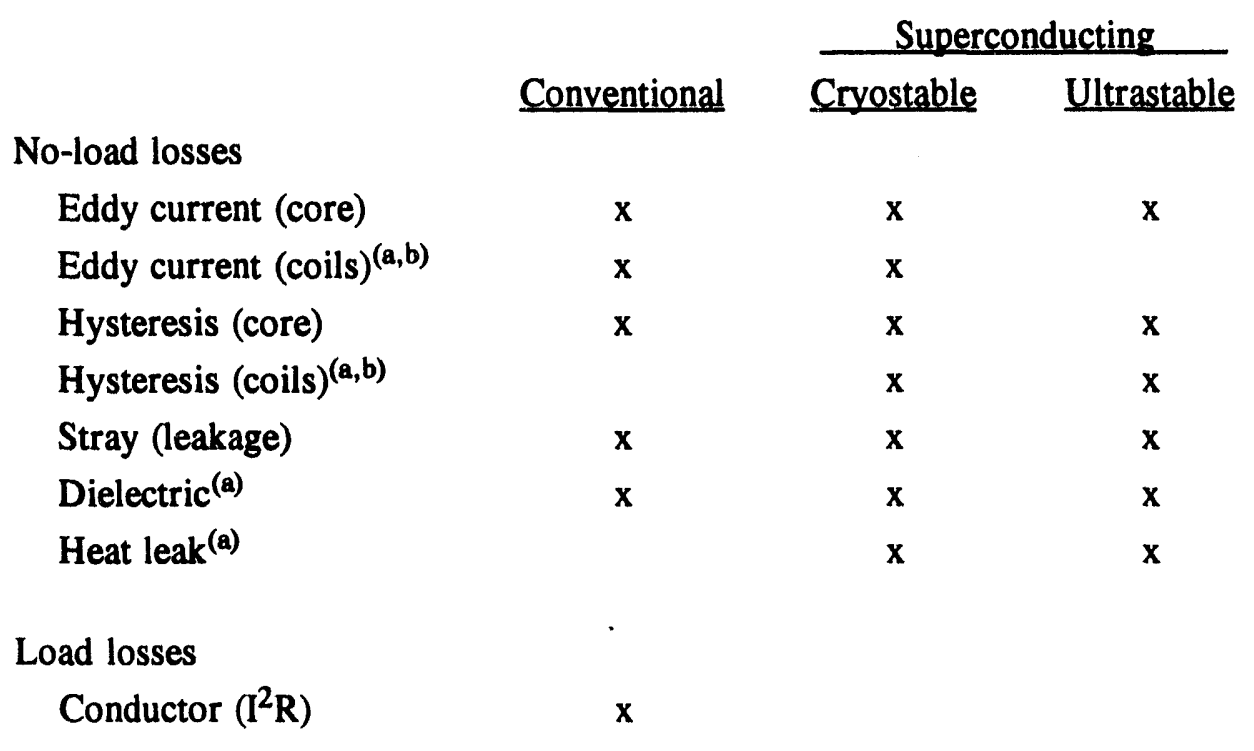

(a) Removed by cryocooler in superconducting designs.

(b) Self-field influence calculated at design current. 
life-cycle cost. The minimum life-cycle cost philosophy was adopted as the overall transformer design criterion. As such, the performance results reported in the study do not represent maximum attainable efficiency values but are the values obtained for designs optimized on a life-cycle cost basi.

We found that optimized superconducting transformer designs have higher efficiencies than their conventional counterparts at the same megavolt-ampere (MVA) ratings. This is primarily because load losses in the windings are eliminated. Changing from a conventional design to a superconducting design involves much more than simply replacing the copper windings with superconducting windings. Numerous design tradeoffs come into play. The most important of these are the peak core flux density and the core dimensions (and leakage losses), which together affect the required number of winding turns, the core radius, the leakage flux, core losses, core cost, and windings costs.

Figure 2.1 shows the efficiency of designs with minimum life-cycle cost for the base case grid applications (autotransformers and step-down transformers). For all transformer types (cryostable, ultrastable, and conventional) and sizes, the optimal amorphous core designs have higher efficiencies than the conventional laminated core designs. Regardless of core technology, the efficiencies of the ultrastable designs are the highest for any MVA rating, followed by the cryostable and conventional designs (for the base case material parameters). Furthermore, as expected, the efficiencies of all types of transformers are high ar with increasing rating. The 30-MVA step-down transformer is considerably less efficient than the autotransformers. This lower efficiency occurs not only because of its lower MVA rating but also because, in the step-down transformers, all the energy must be

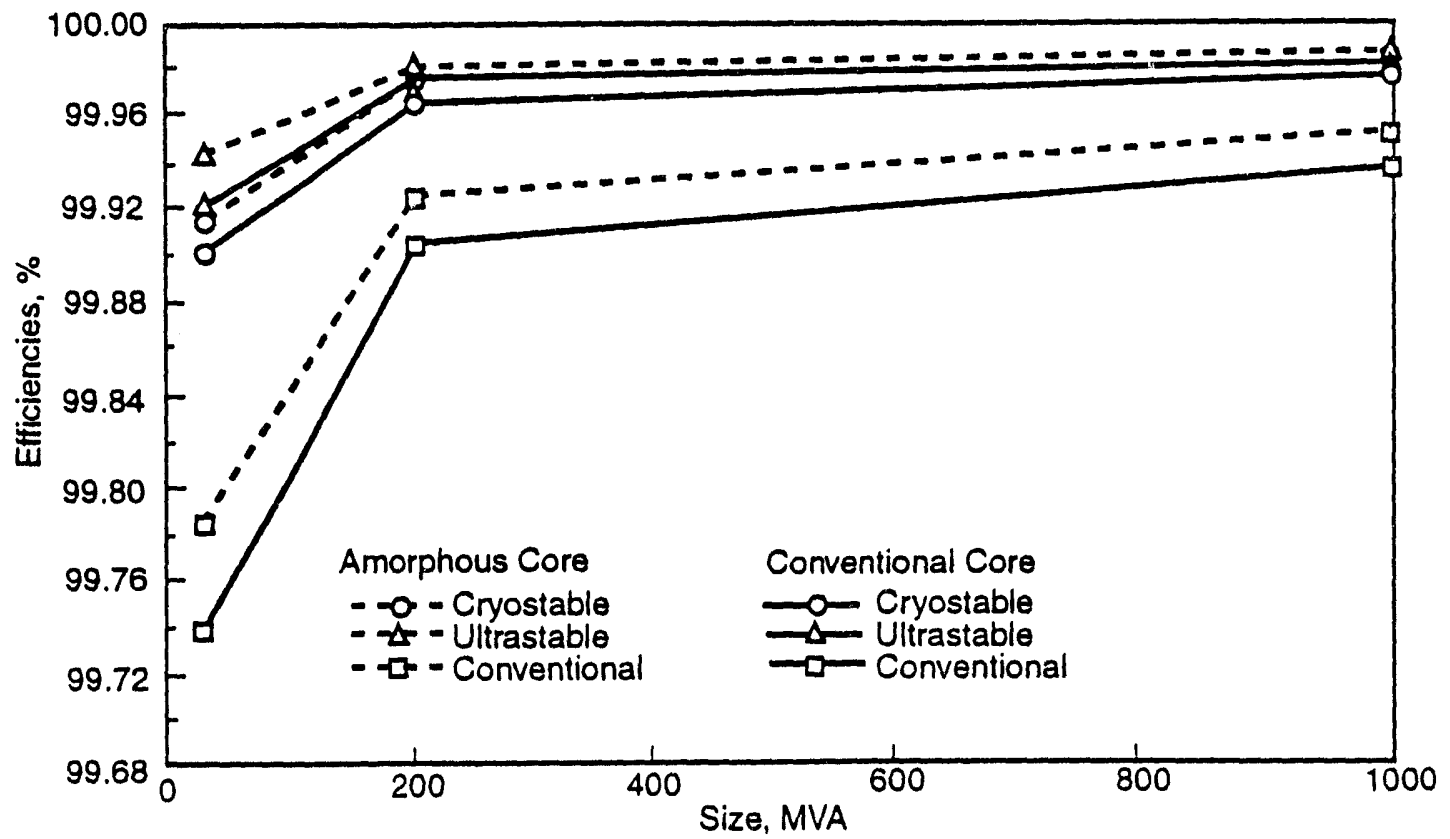

Figure 2.1. Efficiency of Grid Transformers Designed for Lowest Life-Cycle Cost 
transferred through the core and windings of the transformer. By contrast, in an autotransformer, some of the energy is passed through the interconnected primary and secondary windings.

The key conclusions from the transformer design/performance analysis are summarized below.

- Amorphous cores dramatically decrease the core losses in conventional iransformers. This occurs because the increased resistivity of the amorphous core material reduces the eddy currents in the core and the amorphous structure greatly reduces the hysteresis losses.

- For the same MVA rating in a conventional transformer, the optimal amorphous core design will have a larger core than the comparable conventional core design. Amorphous cores have lower possible flux densities and a lower stacking factor than laminated silicon-steel cores. Thus, larger cores are to be expected.

- In superconducting designs with conventional cores, the peak core flux density will typically be greater than in the comparable conventional designs with conventional cores. A higher peak core flux density, combined with higher possible operating current densities in superconducting winding, results in smaller core radii and much reduced winding volumes. Some of the losses inside the cryostat (eddy currents in the stability material and hysteresis losses in the superconducting material), are induced, in part, by the leakage flux. The leakage flux then increases with core flux density. However, the decreased volume more than compensates for the increase in the per unit of mass losses.

- For the base case assumptions, cryostable designs have much lower material requirements than comparable conventional designs and slightly lower material requirements than ultrastable designs. The operating current density of the ultrastable and cryostable (including superconducting and stability material) designs is much higher than the conventional designs. Because the operating current density of the superconductor is much higher than that of the copper in conventional designs, the core window areas and winding volumes for the superconducting designs are much smaller. This higher current density results in designs that typically have more than twice the number of turns of the comparable conventional design. The higher number of turns allows the core radii to be reduced relative to the conventional designs with only a slight increase in core flux density. Thus, regardless of core type, the superconducting designs will have much smaller cores and associated core losses than conventional transformer designs.

- The base case ultrastable designs have much higher HTS material requirements than the cryostable designs because of the large operating safety factor used in base case ultrastable designs. Decreasing the required operating safety factor (possibly through the use of external current-limiting devices) would result in significantly smaller, more efficient ultrastable designs.

- The stabilizer requirements (64\% to $88 \%$ of the winding weights for the base case designs) increase roughly proportional to the $4 / 3$ power of the current. Thus, transformers with larger ratings (and currents) will require a higher percentage of stabilizer material and, therefore, eddy currents will typically represent a larger fraction of the total losses. 


\subsection{System Integration Issues}

A primary advantage of HTS over low-temperature superconducting (LTS) systems is the opportunity to use liquid nitrogen as the refrigerant. Cheaper, more efficient, and simpler than liquid helium systems needed for LTS, liquid nitrogen, with a 77-K boiler point at ambient pressure, offers many advantages from a user-acceptance standpoint. However, to operate reliably at this temperature, the critical temperature, Tc, of the superconductor must be higher than $77 \mathrm{~K}$ at the maximum design current density and magnetic field.

It appears that with proper design, future HTS transformers could be installed on electric utility systems as direct substitutions for conventional equipment. The principal issue in designing the HTS transformer as a "black box" equivalent to a conventional transformer appears to be the real-time management of conditions that maintain the superconductivity of the winding subsystem. Although the subsystems needed would be very similar to those used for conventional transformers, they would differ depending on the HTS transformer type. For example, with the HTS ultrastable transformer, additional current limiters and control coordination might be needed to manage startup inrush currents and high-magnitude, low-probability fault currents, as well as other transients that could quench the coils.

The HTS transformer does not appear to present unsurmountable problems in installation and operation, although some design and engineering effort will be required. However, the operation and maintenance of refrigerant subsystems could present a major obstacle to utility acceptance of HTS transformers. The utilities have no operating experience in this area and, historically, have not been quick to adopt new subsystems.

It is likely that the small sizes and low mass of HTS transformers will eventually have a profound impact on how a utility plans for spare transformers within its system. Where high-capacity conventional transformers require specialized vehicles for transportation and are extremely difficult to move, many comparable capacity HTS transformers could be expediently moved on flatbed trucks. Such capability could greatly reduce the number of spare transformers required by utilities because spares could be quickly moved over a larger geographic area.

Market acceptance of a new technology is not based solely on technical performance and economic issues. Utility acceptance of HTS transformers, even after they might be considered to be fully engineered, will probably be conditioned heavily by factors such as incompletely demonstrated reliability, lack of user experience, low confidence in a new technology and, in general, management conservatism. The utility industry has historically dealt with such issues with extreme caution and has required extensive field testing and evaluation of hardware to resolve these reservations.

Therefore, as a first step in demonstrating the potential practicality of HTS transformers, it is recommended that a liquid nitrogen-cooled cryo-resistive unit be built and operated for several years by a host utility under typical service conditions. Such a unit could be used to develop and demonstrate a viable cryogenic subsystem for a future HTS transformer. In this way, experience could be generated with the cryogenic components of the device to provide a basis of utility confidence while the conductor technology is still on its pathway to maturity. The independent and 
parallel development of both the cryosystem and conductor technology would reduce the technological risk of incorporating them in a prototype HTS transformer at some later date.

As a purely experimental unit, such a transformer would probably be more expensive to purchase and operate than a conventional transformer of the same capacity. However, the unit could be designed to embody some of the values anticipated in future HTS transformers such as reduced size and weight. With these features, the unit might be used on several utility systems to demonstrate the mobility advantage and to broaden the base of operating experience that may be critical to utility acceptance of cryogenic technology. The computer performance and cost-estimating codes developed in this study could be readily adapted to the task of optimizing cryo-resistive transformer designs.

\subsection{Cost Characterization}

High-temperature superconducting transformers have the potential for significant life-cycle cost savings relative to conventional transformers for all applications evaluated to date. The life-cycle costs of the conventional designs ranged from 1.6 to 2.3 times higher than the best comparable HTS designs. Unlike most energy-saving technologies, where initial costs are high but energy costs are low, the HTS transformers achieve their life-cycle cost advantages by both lower initial capital costs and lower energy costs. The lower capital costs of the HTS transformers occur primarily because the HTS transformers are significantly smaller than conventional transformers. A breakdown of the lifecycle costs is shown in Figure 2.2 for a 1000-MVA autotransformer case. This breakdown is fairly representative of the results for other applications.

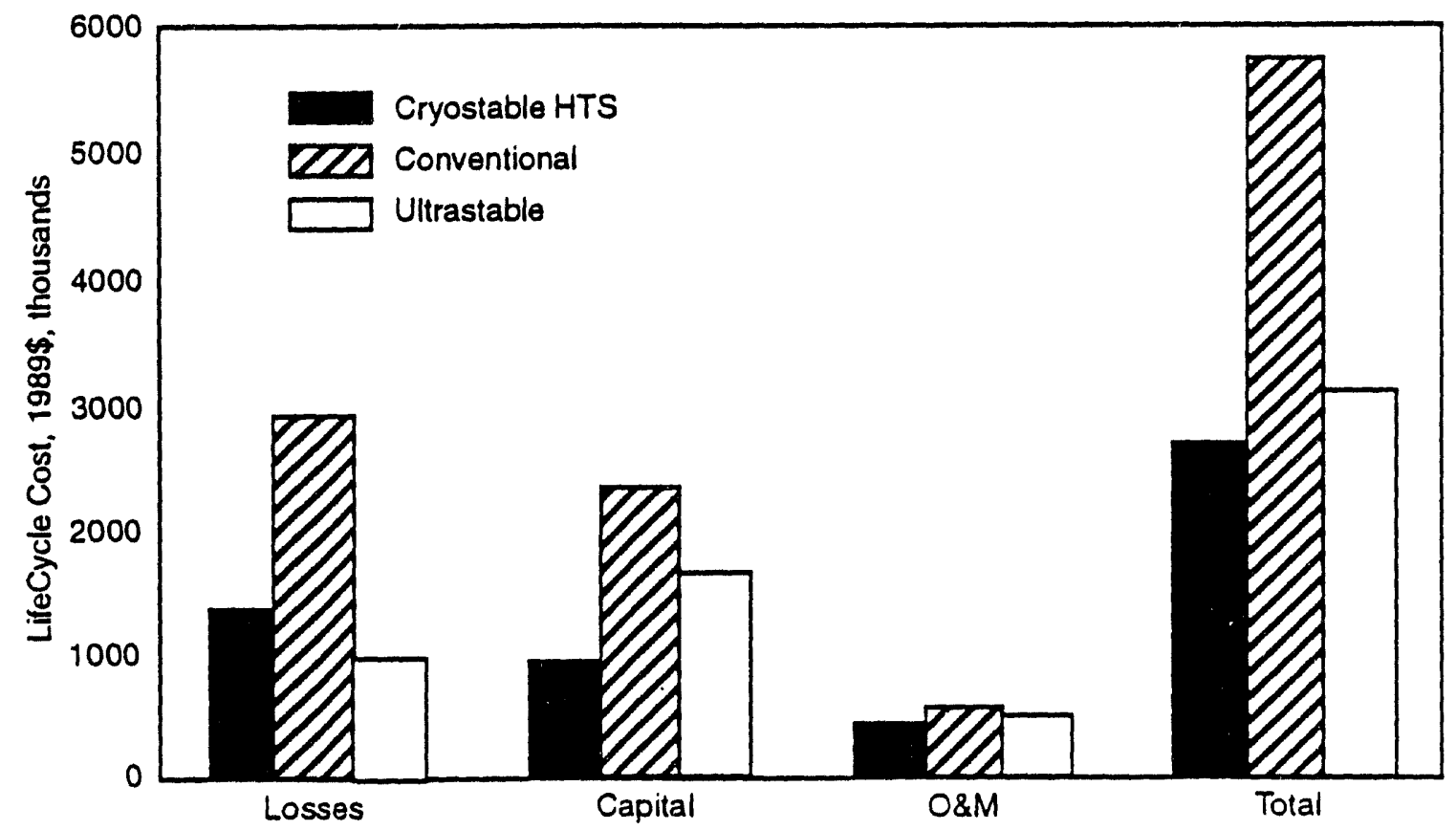

Figure 2.2. Life-Cycle Cost Comparison, 1000-MVA Grid, Silicon-Steel Core 
We investigated the use of amorphous cores in both conventional and HTS transformer designs. It appears that the cost impacts of amorphous cores are similar between the two types of transformers, and that future incorporation of amorphous cores in power transformers would not change the relative economic advantages that HTS transformers show compared with conventional transformers.

For the base case design assumptions, cryostable HTS (HTS-CS) transformers have a minimal (10\% to 20\%) life-cycle cost advantage over ultrastable HTS (HTS-US) transformers.

The key life-cycle cost conclusions are summarized below.

- Both the HTS-CS and the HTS-US technologies appear to show significant life-cycle cost advantages relative to conventional transformers. Compared with the conventional transformers, the HTS technologies appear to have the potential for both lower energy losses and lower capital costs.

- For the base case materials assumptions, the HTS-CS has a slight life-cycle cost advantage (4\% to $14 \%$ ) over the HTS-US technology. However, within the overall uncertainty of this study, neither approach to designing HTS transformers appears to have a clear economic superiority for the base case materials assumptions.

- The use of amorphous cores in transformer designs does not appear to impact the economic attractiveness of HTS transformers relative to conventional transformers.

\subsection{Sensitivity Analyses}

Sensitivity analyses are important because they provide insights into the interaction among materials characteristics, design parameters, cost, and market penetration. Sensitivity analyses were focused on two areas very important in the design of HTS transformers: the critical current density of the HTS materials and the effects of self-fields. Our results indicate that the effect of increasing the critical current from low values to higher values does not always represent an improvement in the transformer design. In actuality, an optimal critical current (from a life-cycle cost standpoint) will exist for transformers. The examination of the self-field impacts indicates that this is a critical performance parameter that can dramatically impact the losses, efficiency, and costs of HTS transformers.

The key conclusions are described below.

- For the base case materials parameters, the HTS-CS technology appears to be economically superior to the HTS-US technology at values of $\mathrm{J}_{\mathrm{c}}$ below about $10^{5} \mathrm{~A} / \mathrm{cm}^{2}$.

- The results of this study indicate that very high levels of $J_{c}$ should not be required for designing a cryostable transformer economically superior to a conventional transformer. For the economic conditions assumed in this study, it appears possible to design cost-effective cryostable transformers at values of $J_{c}$ less than $10^{4} \mathrm{~A} / \mathrm{cm}^{2}$. 
- For ultrastable transformers to compete with conventional transformers, the critical current density of the material should be at least $10^{4} \mathrm{~A} / \mathrm{cm}^{2}$. The viability appears to become better for slightly higher values of $\mathrm{J}_{\mathrm{c}}$, but beyond $\mathrm{J}_{\mathrm{c}}=10^{5} \mathrm{~A} / \mathrm{cm}^{2}$, we actually found decreasing benefits from using such material. This suggests that high-temperature superconductor research efforts should first target the development of materials with $J_{c}$ less than $10^{6} \mathrm{~A} / \mathrm{cm}^{2}$ for power transformer applications.

- The existence of a knee in the performance and life-cycle cost curves, as a function of critical current density, is dependent primarily on the fields to which the conductors are exposed. Thus, if the self-fields determined by the model are not representative of actual transformers that would be constructed in the future (which may, in fact, be designed with the intent of minimizing the negative effects of high self-fields), the location of the knee would be different. It is likely that a knee will always exist at some $J_{c}$, but good engineering design may be able to shift the knee to a higher $J_{c}$ than can possibly be attained with high-temperature superconductors. Therefore, by considering only attainable values of $J_{c}$, it would appear that further increases in $J_{c}$ will always result in further increases in efficiency and decreases in life-cycle cost.

- For superconducting transformers to be more efficient and cost-effective than conventional designs, great care must be exercised in designing transformers that will minimize the fields to which the conductors are exposed. Minimization of the field (leakage or self-field) is likely to be the single most important factor in the design and ultimate performance of superconducting transformers and other alternating current (ac) superconducting devices. Final resolution of this issue requires detailed design and analysis, prototype testing, or both.

- The superconducting material parameters tend to drive the design and performance of the HTS transformers. By changing the assumed filament diameter of the HTS material from $50 \mu \mathrm{m}$ to $0.5 \mu \mathrm{m}$ (still larger than what is possible with many ceramics), we showed that the ultrastable design losses decreased by up to $64 \%$ and the life-cycle cost decreased by $30 \%$. The effect on the cryostable design was much less significant; losses and life-cycle costs changed less than $15 \%$. Thus, it is impossible to say whether cryostable or ultrastable designs will have the ultimate cost and performance advantage until the material parameters are known. However, analyses such as these can point to areas where research will provide the most beneficial results.

\subsection{Market Penetration/Benefits to the Nation}

Two market penetration scenarios were used to evaluate the market potential of HTS transformer designs: a base case and a sensitivity case. For the base case, the initial year of market sales was assumed to be 2000 and the final year of market sales was assumed to be 2020 . Because the benefits of adopting HTS transformers extend beyond the final year of sales, we continued to measure benefits to the year 2060. For the sensitivity case, the initial year of market penetration remained 2000, but the final year of market penetration was extended to 2030 and the measurement of benefits continued until 2070.

The results of the market penetration analysis indicate that the potential economic savings to the nation from developing HTS transformers could be quite large. For the base case market penetration 
analysis, the savings estimates ranged from $\$ 15$ to $\$ 17$ billion (present value, 1989 dollars) depending on the type of HTS transformer evaluated. In the sensitivity analysis, which considered a longer time period, the savings ranged from $\$ 25$ to $\$ 28$ billion (present value, 1989 dollars).

The benefits of adopting HTS transformers include not only the dollar savings (in capital, operating and maintenance, and energy costs) bu: also the reduced amount of energy that must be generated in the future. Based on the market penetration analysis, we evaluated the amount of energy saved by adopting HTS transformer designs. The results indicated that developing HTS transformers could result in cumulative energy savings of between 90 and 300 billion $\mathrm{kWh}$ (approximately 1 to 3 quads of primary energy) over the analysis period. 


\subsection{Design and Performance Characterization}

The conversion of voltage and current levels in an electrical power system requires the use of transformers; these components constitute a significant portion of the total investment in the electrical power system. They also have an important effect on the overall performance and efficiency of the power system. Accordingly, transformer design influences the overall system layout, power conversion efficiency, and cost. Because of the interdependence and interaction of transformer design parameters, judicious tradeoffs are necessary to achieve optimum designs that minimize life-cycle . cost.

The performance of large power transformers can be enhanced through the use of superconductor technology. Several design studies based on low-temperature superconductors support this belief (McFee 1961; Wilkinson 1966; Lurch 1969; Westinghouse Electric Corporation 1982). The estimated benefits, however, were considered to be marginal; many designs were not cost-effective (Borchards 1966; Harrowell 1970). Technical problems from operating transformer components at temperatures near absolute zero were found to require complex and expensive systems. However, the application of high-temperature superconductors offers a means for avoiding these limitations and has significant potential for improving transformer design, performance, and cost.

This chapter provides an overview of transformer design issues, a discussion of how these issues apply to the design of HTS transformers, and a description of the approach used in the transformer design analysis. To provide general background for the basis of formulating the transformer design model, the basic principles involved in transformer design are discussed. Following is a section that discusses the various key equations used in the design algorithm (code structure and methodology are discussed in Appendix B). Base case results and conclusions appear in the final sections.

\subsection{Transformer Fundamentals}

In simple terms, the basic transformer takes electrical power from one voltage and current level at its input terminals and "transforms" it to a different level at its output terminals. This conversion is accomplished in such a manner that the power, which is the product of the voltage and current, is approximately unchanged between the input and output terminals. Modern transformers are highly effective and energy-efficient devices. Large power transformers may operate with efficiencies greater than $98 \%$.

Power transformers are used to convert the relatively low-voltage, high-current power from generators to high-voltage, low-current transmission power. This conversion helps to reduce the energy lost transmitting power over long distances to consumers. Other transformers then step down the voltage to lower levels for subtransmission and distribution, and then again to the voltage levels used by electrical appliances and equipment.

The following section provides a brief description of the way transformers operate, along with some relevant information useful in understanding some of the tradeoffs made in designing a transformer. 


\subsubsection{Ideal Transformer Theory}

The basic transformer consists of two conductor coils wound around a common core of high magnetic permeability material. Magnetic material such as silicon steel or nickel alloy is often used for this purpose. Magnetic flux is induced in the transformer core by current flowing in the primary windings. This magnetic flux induced in the transformer core, in turn induces current to flow in the secondary windings. The coils are wound and connected so that current flowing into the primary produces current flowing out of the secondary.

For transformer analysis, it is often convenient to begin with idealized relationships rather than a complete, but more complex, transformer model. These ideal transformer relationships closely model the operation of an actual transformer.

Because the coils of the transformer are wound around a steel core of very high magnetic permeability, we can assume that the magnetic field outside the core is negligible. If this is the case, then every turn of both transformer coils is linked by the same magnetic flux. This assumption (not entirely true in an actual transformer) ignores "leakage flux," i.e., flux that links only one winding or a portion of a winding and not the other.

In an ac circuit, the magnetic flux has sinusoidal variation with time. By Faraday's law, this alternating flux produces a sinusoidal voltage in each turn of the coils. The voltage induced in each turn of the winding is called "volts-per-turn" and is an important parameter in transformer design.

Both windings of the ideal transformer have the same volts-per-turn. The terminal voltage of each coil is simply the volts-per-turn multiplied by the total number of turns in the coil. It follows that the transformer voltage ratio is equal to the coil turns ratio. This may be expressed as

$$
\frac{V_{2}}{V_{1}}=\frac{N_{2}}{N_{1}}
$$

where $V_{1}$ and $V_{2}$ are the terminal voltage of windings 1 and 2 (the primary and secondary windings, respectively), and $N_{1}$ and $N_{2}$ are the number of turns for the two windings. The above expression is thc first of three "ideal transformer equations" that describe the operation of a hypothetical ideal transformer.

Another ided transformer relationship follows from the assumption that no energy is stored or lost in an ideal transformer. Thus, the power entering the primary terminals is exactly equal to the power leaving the secondary terminals. Expressed mathematically, this is

$$
P_{1}=I_{1} V_{1}=I_{2} V_{2}=P_{2}
$$

where $P_{1}$ and $P_{2}$ are the power into the primary and out of the secondary, respectively, and $V_{1}$ and $I_{1}$ and $V_{2}$ and $I_{2}$ are the terminal voltages and currents, respectively. As a result of these two "ideal" 
transformer relationships, the third ideal transformer relationship is one in which winding currents are inversely proportional to the turns ratio; that is,

$$
\frac{I_{1}}{I_{2}}=\frac{N_{2}}{N_{1}}
$$

In summary, the ideal transformer changes the voltage between the winding terminals by the ratio of the coil turns and changes the current by an inverse ratio. The ideal transformer is perfectly lossless, with the input power equal to the output power.

Of course, actual transformers are not ideal. Although the performance of modern large power transformers closely approximates the ideal relationships, they are not completely lossless. There is a voltage drop through the transformer coils under load, so the voltage ratio is not exactly equal to the turns ratio, and an excitation current proportional to the supply voltage flows into the transformer even when there is no load current. Thus, the current ratio does not quite match the ideal relationship.

The transformer design engineer must take these factors and other performance criteria into account. Acceptable voltage regulation characteristics, losses, and inupedance must be designed within acceptable limits while minimizing total life-cycle cost. The more important transformer loss mechanisms, basic design considerations and tradeoffs, and the application of superconductive technology to power transformers will be discussed later.

\subsubsection{Issues Related to Superconductive Transformer Design}

By eliminating the resistance losses in the windings, which is a major loss in the conventional transformer, superconductive transformer windings offer the potential for bringing the transformer performance closer to the ideal relationships.

Achieving superconductivity is constrained by three interrelated factors: the temperature of the superconductive material, the strength of the magnetic field in which the conductor is operating, and the current density. Exceeding a certain "critical" level in any one of these factors "quenches" the superconductor; that is, causes it to return to "normal" conductivity. Furthermore, as the critical value of one of these factors is approached, the critical values of the other two are reduced.

Before 1986, the highest critical temperature, $T_{c}$, for any known superconductor was about $23 \mathrm{~K}$ $\left(23^{\circ} \mathrm{C}\right.$ above absolute zero). These low- $\mathrm{T}_{\mathrm{o}}$ superconductors were cooled by liquid helium, which is expensive and difficult to handle. For example, helium boils at $4.2 \mathrm{~K}$ and requires a very complex and energy-intensive refrigeration system. In addition, the scarcity of helium increases acquisition cost. Recently, superconductors with $\mathrm{T}_{\mathrm{c}}$ exceeding $120 \mathrm{~K}$ have been discovered. These superconductors can be cooled with liquid nitrogen, which boils at $77 \mathrm{~K}$ and is relatively inexpensive.

The second critical factor affects the ability of the material to superconduct in a magnetic field. The stronger the magnetic fields at a given temperature, the fewer the available superconductive electrons. For every temperature, there is an upper critical field, where the material is in a 
nonsuperconductive state. For new generation high-temperature superconductors, this field has been extrapolated to be over 100 tesla at liquid nitrogen temperature (Malozemoff et al. 1987). This field intensity is well above any conceivable stray field in an iron core transformer.

The third critical factor influencing superconductivity is the material critical current density, which is the maximum current in a given cross-sectional area of material. The critical current density, $\mathrm{J}_{\mathrm{c}}$, is the point where excessive current prevents the material from entering a superconductive state. The $J_{c}$ will be reduced if either the temperature or the applied magnetic field increases. The critical current density is important because, as $J_{c}$ increases, less superconductive material is required to carry the necessary current.

A multistrand, bismuth-based wire has been produced with a critical current of $13,000 \mathrm{~A} / \mathrm{cm}^{2}$ at $77 \mathrm{~K}$ in zero field (Superconductor Industry 1991). With further development, critical current densities as high as $10^{7} \mathrm{~A} / \mathrm{cm}^{2}$ may be possible in high-temperature superconductors (Malozemoff et al. 1987).

Achieving the necessary critical factors for economic designs is but one problem facing the superconductor material scientist. High-temperature ceramic superconductors have proven difficult to fabricate into workable forms. They are brittle, which is expected in ceramic material, and they exhibit an anisotropic critical current density; that is, the ceramic crystal is able to carry many times the current in one direction than it will in another. Bulk superconductors made from sintered powders are composed of numerous small crystals oriented at random, interspersed with normally conductive regions. This results in relatively low critical current densities. Advances in material fabrication techniques should yield higher critical current densities in usable forms.

Materials scientists are working to develop flexible conductors that are monocrystalline and oriented to carry current in the preferred direction. In this study, we are assuming that problems associated with materials development will eventually be solved, yielding viable conductor designs. To cover the complete range of potentially available critical current density materials, we examine optimum transformer designs with $\mathrm{J}_{\mathrm{c}}$ from $10^{3}$ to $10^{7} \mathrm{~A} / \mathrm{cm}^{2}$.

High-temperature ceramic superconductors may be configured differently from low-T superconductor wires (filaments bundled in strands of metal matrix). These new material configurations, such as thin ribbons, could use the material's ceramic properties advantageously and improve the operating characteristics of the wire (such as minimizing the self-induced magnetic field with a more optimum configuration). Because there is no practical experience with such material configurations, the low- $T_{c}$ superconductor wire configuration is assumed in this study.

\subsection{Major Transformer Loss Mechanisms - Load Losses and No-Load Losses}

Although the ideal transformer relationships provide a close approximation of transformer behavior, a study of transformer performance must take into account the departures from perfection. The power loss in a transformer, which results in generated heat, comprises no-load losses and load losses, as described below. 
The no-load loss is a constant, present whenever the transformer is energized. This loss is a function of the voltage applied to the transformer terminals, which is relatively unchanged.

The load loss, on the other hand, is a variable loss that is a function of the load current. This loss is negligible when the transformer is lightly loaded, but is substantial when the transformer is supplying power at its full capacity. For some transformer applications, the load current can change substantially throughout the day, which results in a wide variation of these load losses.

In a conventional transformer, maximum power efficiency occurs when the no-load losses equal the load losses. However, maximum efficiency designs may not necessarily yield the most economic designs. Increasing the efficiency usually results in an increase in the capital cost of the transformer. At some point, the incremental increase in capital cost exceeds the value of the loss reduction that can be obtained. Therefore, with large power transformers, the standard practice is to design the unit to operate most economically rather than most efficiently. The exact design operating point is determined by the value the utility places on power and energy losses. This determination includes factors related to the resources available to the utility, the cost of purchasing or generating power during different situations, and the transformer loading duty.

The total transformer losses may be expressed as

$$
P_{\text {Loes }}=P_{L}+P_{N L}
$$

$P_{\text {Lom }}$ is the total transformer loss, $P_{\mathrm{L}}$ is the load loss, and $P_{\mathrm{NL}}$ is the no-load loss term. The load loss term may be expressed for the conventional transformer as

$$
P_{L}=P_{C u}=\frac{\sum}{\text { all } i} I_{i}^{2} R_{i}
$$

where $\quad I_{i}=$ current in winding $i$

$R_{i}=$ resistance of winding $i$.

The load loss term, also called the copper loss, is proportional to the square of the load current. This loss term is relevant only for conventional transformers.

With superconductive transformers, there is a small dependence of the losses on the load current, caused by the self magnetic field induced by the load current. This self-field combines with the leakage field (which is not a function of the load current) to drive losses in the superconductive windings. Our model combines this loss term with the no-load losses, thereby treating the superconductive transformer as if there were no dependence of losses on the load current.

The self-field dependent losses of the superconductive transformer vary much less as a function of load current than the copper losses in conventional transformers. Modeling superconductor losses as load loss terms would necessitate new economic algorithms to assess the impacts of these losses on the transformer design goals, specifications, and requirements. Additionally, for most of the transformer applications considered, the load current seldom drops below a significant portion of the 
full capacity current. Therefore, we have included the self-field dependent losses as no-load loss terms. This assumption tends to provide more conservative results for the superconductive transformer designs, especially for applications in which large load variations are encountered.

The no-load loss term incorporates a number of components. This term may be expressed for the conventional transformer as

$$
P_{\text {NT }}=P_{P_{e}}+P_{\text {Leat }}+P_{d}+P_{\text {Eddy }}
$$

For the superconductive transformer, it may be expressed as

$$
P_{N L}=P_{F e}+P_{\text {Leat }}+\left(P_{d}+P_{E d d y}+P_{\text {tyw }}\right)\left(1+1 / h_{t h}\right)+\left(P_{\text {Heat Leak }}\right) / h_{t h}
$$

where

$$
\begin{aligned}
\mathbf{P}_{\mathrm{F}_{0}} & =\text { iron loss due to hysteresis and eddy currents in the core } \\
\mathbf{P}_{\mathrm{L}_{\text {Lak }}}= & \text { stray loss due to leakage flux outside the cryostat } \\
\mathbf{P}_{\mathrm{d}} & =\text { dielectric loss in the transformer insulation } \\
\mathbf{P}_{\mathrm{Eddy}} & =\text { eddy-current loss induced in the winding stability material } \\
\mathbf{P}_{\mathrm{Hyt}} & =\text { hysteresis loss in the superconductive windings } \\
\mathbf{P}_{\text {Heat Lak }}= & \text { external heat that leaks into the cryostat and becomes a load on the } \\
& \text { refrigeration system } \\
\mathrm{h}_{\mathrm{th}}= & \text { thermal efficiency of the refrigeration system, commonly called the } \\
& \text { coefficient of performance (COP). }
\end{aligned}
$$

For both the conventional and superconductive transformer designs, the dominating term in the no-load loss expression is normally $\mathbf{P}_{\mathrm{Fo}}$. However, the other loss mechanisms listed may contribute significantly (depending on the type of design considered) and are addressed in detail in the following subsections.

\subsubsection{Transformer Load Losses}

The actual conventional transformer departs from the ideal in that there is a voltage (IR) drop due to the winding resistance. The amount of current that flows through the windings of the transformer is directly proportional to the transformer power demand. Power is dissipated in the conventional transformer because of resistance to the flow of current in the copper windings. These copper losses are the load losses previously mentioned and have an $I^{2} R$ relationship.

Because the current through the transformer is determined by the load, the $I^{2} R$ relationship specifies that for a given transformer configuration, the load losses can be reduced only by reducing the resistance of the winding conductors. Resistance can be lowered either by using larger wire (increasing the amount of materials required and hence capital cost) or by using conductor material with a lower resistivity.

The latter method of reducing transformer losses is the primary motivation for pursuing the development of a superconductive power transformer. In a conventional transformer, the load loss 
component typically accounts for more than $50 \%$ of the total transformer losses. This loss term can be greatly reduced through the use of superconductive transformer windings.

Although there is no resistance to current flow in a superconductive transformer winding, there are energy losses. The magnetic field present causes hysteresis and eddy-current losses in the superconductive material and stabilizer, respectively. These losses are somewhat dependent on the transformer load current; however, the self-induced fields in adjacent windings produce oppositely oriented fields, which tend to cancel. In addition to the self-field, the leakage field (which is not a function of the load current) contributes to the loss-causing fields seen by the conductor. The combined effect is hysteresis and eddy-current losses that are negligible when compared to the load losses in a conventional transformer. Thus, the overall transformer losses are significantly reduced. However, because the power dissipated in a superconductive transformer must be removed through a cryogenic refrigeration system, an additional energy penalty is added to these losses. Thus, all superconducting winding losses must be minimized to reduce the overall power consumed while the unit is operating.

\subsubsection{Transformer No-Load Losses}

Transformer no-load losses are explained in the following subsections.

\subsubsection{Core Loss Component}

The core in an actual transformer is not infinitely permeable, as was assumed in the ideal transformer relationships. It requires magnetizing current to establish the magnetic flux. In addition, there are internal losses in the core as the flux varies with time. These losses in the transformer core constitute the dominant no-load loss term in the conventional transformer. The two main core loss components are called hysteresis and eddy-current losses.

\subsubsection{Hysteresis Loss in the Transformer Core}

When the magnetic material in the transformer core alternates through a complete cycle, magnetic domains within the material change their orientations to remain aligned with the magnetic field induced by the transformer windings. As the magnetic core material tends to resist this realignment, a magnetizing force is continually exerted on the magnetic domains within the core. The power required to overcome domain inertia and change magnetic alignment is termed the hysteresis loss within the transformer core.

In ac applications, this process is repeated continuously. The total hysteresis loss is proportional to the hysteretic loss per cycle, the operating frequency, and the amount of core material.

The magnitude of the hysteresis loss is dependent on the flux density within the transformer core, which, in turn, depends on the terminal voltage of the transformer, according to Faraday's law, $E=N(d \Phi / d t)$, where $N$ represents the number of turns, $\Phi$ is the flux, and $E$ is the voltage induced by the changing flux. For a transformer, this relation may be expressed as 


$$
B=\frac{E}{\sqrt{2} \pi f N A}
$$

where $\quad B=$ the peak core magnetic flux density $(T)$

$E=$ the terminal voltage of the coil $(\mathrm{V}, \mathrm{rms})$

$\mathrm{f}=$ the transformer operating frequency $(\mathrm{Hz})$

$\mathrm{N}=$ the number of windings on the coil

$A=$ the cross-sectional area of the core $\left(\mathrm{m}^{2}\right)$.

Slight increases in the terminal voltage of the transformer can push the core iron into saturation and greatly increase the core losses. Hysteresis losses can be reduced by operating the transformer near the saturation flux density. However, for a given flux, reducing the flux density increases the transformer core volume, potentially yielding increased transformer capital cost, weight, and size.

Hysteretic losses may be reduced also by selecting core material with a low coercive force. These low-loss core materials, however, typically saturate at a relatively low-flux density, again necessitating an increase in the transformer size and capital cost.

\subsubsection{Eddy-Current Loss in the Transformer Core}

The hysteresis loss is only part of the energy loss encountered when the core is subjected to an alternating field. The changing flux induces small circulating currents, known as eddy currents, to flow within the core material. As they circulate through resistive core material, these currents cause $I^{2} R$ losses.

As with the hysteresis core loss component, the magnitude of the eddy currents depends upon the flux density within the core. Operation at a reduced flux density reduces the eddy currents, again at the expense of increased transformer dimensions.

Other factors that influence the eddy-current losses are the operating frequency, the thickness of the lamination within the core, and the resistivity of the core material. Currents circulating in the core can be reduced by reducing the thickness of the core lamination and by increasing the resistivity of the core material. The disadvantages of these measures, however, are that the transformer dimensions, capital cost, and fabrication difficulties increase.

The resistivity of the core can be increased by alloying, typically with silicon. An important fabrication technique for increasing core resistivity that is beginning to be applied to power transformers is the METGLAS (for "metallic glass") process. This core material is manufactured by rapidly solidifying a spray of molten steel on a cooled rotating drum. This fabrication process prevents organization of the metal into a crystallized structure and yields a metallic glass, or amorphous atomic structure steel, which significantly increases the electrical resistivity of the material and dramatically reduces the eddy-current and hysteresis losses. The drawbacks of the amorphous steel core include increased core cost; difficulty in core fabrication; and a reduction in the saturation flux density. These drawbacks lead to an increase in the overall transformer size and capital cost. 


\subsubsection{Stray Loss Component}

Because the permeability of the core material in the actual physical transformer is not infinite, some of the flux will not be contained within the core. This flux, therefore, will not couple both windings, and the energy associated with this flux is lost. To minimize capital costs, transformers are often designed to operate at flux densities very close to the iron saturation level. This reduces the amount of magnetic material required to achieve the same flux. However, operating the core flux density closer to saturation reduces the permeability of the core, thereby increasing the magnetic flux leakage.

In the conventional transformer, no-load losses external to the transformer core itself result primarily from this magnetic flux, which is called the leakage flux. Inductive coupling with structural steel components (which are magnetic materials) can also cause hysteresis and eddy-current losses similar in nature to the core losses. In heavily saturated transformer cores, the magnitude of the stray losses can nearly equal the magnitude of the core losses.

Superconductive transformers also encounter stray losses. However, there are added phenomena of leakage and self-induced magnetic flux causing hysteresis losses in the superconductor.

The losses caused by leakage flux may be decreased by operating at a lower core flux density, or by changing the configuration of the transformer. Interleaving the high- and low-voltage windings is an effective means to minimize leakage flux and is common practice in large power transformers. However, both methods to mitigate excessive leakage flux result in capital cost penalties.

\subsubsection{Eddy-Current Losses in the Transformer Windings}

The windings of the transformer experience eddy-current losses similar in nature to eddy-current losses within the transformer core. Eddy currents in the transformer windings typically result in relatively minor losses for conventional transformers but may be the dominant loss term for cryostable superconductive transformers. The eddy-current losses can be reduced by increasing the resistivity of the winding metal, reducing the winding strand diameter, or reducing the magnetic field the conductor encounters.

Increasing the resistivity of the winding material is not desirable for a conventional transformer, because the windings will experience higher $I^{2} R$ losses as a result. Higher $I^{2} R$ losses tend to greatly increase the overall losses of the transformer, offsetting any reduction in eddy-current losses.

A reduction in the winding strand diameter can significantly reduce the eddy currents in the windings. A type of multibraid conductor known as Litz wire, used to minimize magnetoresistive effects in high magnetic field circuits, is sometimes also employed to minimize eddy-current losses in transformer windings. However, Litz wire tends to be rather expensive relative to conductors typically used, and it occupies more volume than conventional transformer wire. Subdividing the winding conductors into several insulated strands accomplishes the same purpose without most of the additional expense. This has other benefits, including increased conductor flexibility. 
Reducing the transformer leakage flux is another means to reduce eddy-current losses in the windings. However, in a conventional transformer this loss-reduction technique typically increases capital cost in excess of the marginal eddy-current loss reduction benefits obtained.

For a superconductive transformer, more attention must be focused on eddy-current loss within the windings. This loss may dominate all other transformer losses. The large volume of cryostabilizing metal required to maintain conductor temperatures below $T_{0}$ in the event that superconductivity is lost (if the conductor experiences a "quench"), in conjunction with the penalty imposed by the refrigeration system as it removes these eddy-current losses, can require that eddy-current losses be minimized in a viable cryostable superconductive transformer design.

The magnitude of the eddy-current losses in the cryostabilized superconductive windings can be lessened somewhat by fabricating the conductor with an anisotropic resistivity. In low-temperature superconductors, resistive copper-nickel alloy often surrounds the region containing each superconductive filament so that it imparts a higher resistivity in the transverse direction. This has the effect of attenuating the eddy currents, while still allowing free current flow in the copper stabilizer in the event of a quench.

The eddy-current losses in the metal stabilizer of the superconductive transformer windings are amplified by the coefficient of performance of the cryogenic refrigeration system. At liquid nitrogen temperatures, the electrical energy required to remove these thermal loads may be 10 to 20 times the original loss. Therefore, care must be taken to minimize losses that occur within the cryogenic environment of the superconductive transformer.

\subsubsection{Hysteresis Losses in the Superconductive Transformer Windings}

As previously mentioned, superconductors are not completely lossless in ac applications. Shielding currents induced in superconductors produce a magnetic flux equal and opposite to the applied external magnetic field. The direction and magnitudes of these "fluxoids" oscillate synchronously with the time-varying magnetic field. This field is produced from the current in the superconductor and, in the case of a transformer, from an external magnetic field (the leakage field). Energy is used to reorient these superconductive fluxoids in a manner similar to the hysteresis loss in the iron core of the transformer.

The hysteresis loss in the superconductive windings per unit volume is related primarily to the intensity of the magnetic leakage field, the self field, and the diameter of the superconductive filaments. With today's low-temperature superconductor technology, these superconductive filaments may be made small enough to limit the hysteresis to a relatively small percentage of the total losses within the transformer, even when including the power requirements of the refrigeration system. Material technology must be developed to similarly minimize hysteresis losses in high-temperature ceramic superconductors.

\subsubsection{Dielectric Losses in the Transformer Insulation}

Dielectric losses are induced by voltage stresses on the high-voltage transformer insulation. These losses are proportional to the permittivity and loss tangent characteristics of the dielectric material as well as the insulation mean voltage stress. 
The dielectric losses are not usually a significant conventional transformer loss component. However, in a superconductive transformer, they are an additional refrigeration load, which is scaled by the cryogenic refrigeration efficiency.

\subsubsection{Thermal Leakage Losses of the Superconductive Transformer Cryostat}

The remaining component of no-load losses considered involves heat leaking into the cryostat from the ambient environment through a variety of mechanisms. This thermal leakage is an additional load which must be managed by the refrigeration system. The primary mechanisms of thermal penetration are radiant heat in the vacuum insulation vessel and heat conduction through the bushings.

Because a vacuum vessel is used to isolate the cryostat from ambient temperatures, the primary heat transfer, excluding conduction in the bushings, is radiant heat passing through the vacuum space. The amount of heat transferred is relatively small, and could be further reduced by using reflective coatings, such as aluminized Mylar", on the cryostat. However, preliminary analysis revealed that eddy-current losses induced in the reflective coatings would offset the potential reduction in the heat load, so it is not included in the analysis.

The major component of the heat influx is conduction through the transformer bushings. No satisfactory means of reducing this loss mechanism was found.

\subsubsection{Cooling System and Associated Power Consumption for Conventional Transformers}

Active cooling systems are required for large conventional power transformers when the generated losses exceed the natural rejection of heat into the environment, causing the temperature to exceed acceptable operating limits. Various cooling systems are commonly used, depending on the type of installation and the size of the unit. The main distinction between the types of cooling is whether it is progressive cooling (to enable increased power ratings) or constant cooling.

Most substation transformers fit into the first category, where the cooling oil circulates by convection, and various stages of heat removal are activated based on the temperature of the oil . Therefore, a progressive rating scale is used, with the power rating of the transformer increasing with each level of cooling.

Generation station transformers are usually designed with pumped cooling passages and will not operate without the cooling system in operation. There still may be temperature-activated progressive stages of cooling, but there is usually not a self-cooled rating at which the unit can operate in the absence of cooling system power consumption.

Because there are many different types of cooling systems, it is difficult to present a general case. However, the cooling system energy requirement is typically such a small percentage of the

\footnotetext{
- Mylar is a registered trademark of E.I. du Pont de Nemours and Company, Wilmington, Delaware.
} 
total losses that it is not included in the efficiency calculations for the transformer. The power to operate the cooling systems is usually provided by low-voltage station power, available for other operational requirements of the station.

\subsubsection{Superconductive Transformer Cooling}

The superconductive transformer requires the windings to be maintained at cryogenic temperatures. This requirement dictates that the windings be immersed in a cryostat containing suitable cryogenic fluid, maintained at the proper temperature by a cryogenic refrigeration system. The refrigeration system must remove all losses generated within the cryostat, including thermal heat leakage. The refrigerator must also operate essentially independently of the load current flowing through the transformer, because the losses within the cryostat have been modeled as no-load losses.

For the superconductive transformer designs studied for this report, the core is external to the cryostat, with the heat removed by conventional cooling means. For simplicity and accurate comparisons, it is assumed that the cooling system used to remove the heat from core losses is similar to the conventional transformer cooling system. Therefore, as with the complete cooling system for the conventional transformer, the core cooling system is not included in the efficiency calculations. The refrigeration system, on the other hand, is a very significant load and must be included in the analysis. The refrigeration load is assumed to be a constant load and is included as a part of the no-load losses.

The power consumed by the refrigerator is not a separate part of the no-load losses. Each individual loss in the cryostat is penalized by the refrigerator COP, as seen in Equation (3.7). Although the refrigeration system power may not be directly provided from the transformer (using a station power supply instead), it is included as a no-load loss for simplicity and gives an accurate accounting for the cost associated with operating the transformer.

An alternative means of cooling, which may prove economical, is to replace the cryogenic refrigeration system with a reservoir of cryogenic fluid. The cryogen is allowed to boil off through the transformer, providing the necessary cooling. This reservoir could presumably be replenished periodically by an independent supplier. This method might provide advantages of increased reliability (no moving parts) and increased economies of scale.

\subsubsection{Major Transformer Loss Mechanisms}

The load losses are applicable only to the conventional transformers and typically constitute greater than $50 \%$ of the total losses. The conventional transformer no-load losses result primarily from hysteresis and eddy-current losses in the transformer core and are typically the second greatest loss term in conventional transformers. This is not necessarily the case for superconductive designs, where other no-load loss terms may dominate.

Each of the losses identified above has a major influence on transformer design. Efforts to improve transformer efficiency usual", result in increased transformer size, weight, and/or capital cost. Furthermore, efforts to reduce a specific loss component may be counterproductive if other losses increase. 


\subsection{Transformer Design Principles :nd Practices}

The transformer engineer is faced with a set of constraints that must be applied in the design of any transformer. Understanding and accommodating these constraints is crucial in specifying a framework for modeling transformer performance. An important consideration is the output power (the product of the operating voltage and the maximum demand current) that the transformer must be capable of delivering to the load within specified voltage regulation limits. Other factors relate to the minimum efficiency desired, which is associated with the maximum power loss that may be allowed in the transformer, and the maximum temperature rise tolerated in the transformer when it is used in a specific environment.

Important specifications to be considered in designing the transformer are its power-handling capacity, primary and secondary terminal voltage ratings, impedance, efficiency, basic impulse level (BIL), load regulation, and phase arrangement of the windings (e.g., delta, wye). Other important considerations are the transformer size and weight, the value of load and no-load losses in a specific application (which relate to transformer efficiency at a given load), type of cooling to be used, and transformer capital and life-cycle cost.

Engineering large power transformers is an iterative process, in which the values of various design parameters are continually adjusted until an optimized solution is found that meets the design specifications with minimum cost. This is usually accomplished using computer-aided analysis and design techniques. Some of the issues having an important effect on the design will be briefly addressed in this section.

A basic step in designing transformers is selecting the proper core material from the many materials available suitable for transformer cores. Each of these materials has unique core loss, saturation flux density levels, and cost characteristics. A specific core material may exhibit very low iron losses but may saturate at a relatively low-flux density, requiring more core material and increasing the transformer volume. Still another material may demonstrate relatively high iron losses at a given flux density, yet saturate at much higher levels than other materials, yielding smaller and possibly less expensive designs.

The voltage level, power capacity, and number of phases of the transformer are driven by the particular application. Together, the voltage and capacity ratings determine the line currents into the transformer. The current density in the conductor affects the load losses, heat rise, efficiency, size, and cost of the transformer.

The choice of the flux density in the core is very important. It influences the cross-sectional area of the core; the number of windings in the coils; and the size, weight, and cost of the transformer. The choice of the flux density also has a major effect on the no-load losses of the transformer. To minimize size and cost, conventional transformers are typically designed to operate very close to saturation at the expense of an increase in the no-load losses.

For a given flux density, the flux and, thus, the volts-per-turn, are directly proportional to the cross-sectional area of the core. Hence, a large core area allows the use of fewer turns on the windings to support the terminal voltages. Therefore, in searching for the optimal design, the 
transformer designer must trade off conductor volume and associated load losses against iron volume and no-load losses.

The opening in the core through which the windings pass, called the core window area, must be large encugh to accommodate the required number of turns on each of the windings at a given current density, as well as the necessary insulation and cooling space. The transformer performance can typically be improved somewhat by reducing the width and increasing the height of the window. For large power transformers, designers are limited by the maximum dimensions allowable for transporting the transformer to the point of application. Of particular concern is the maximum height allowed, usually limited by railway clearances.

The transformer voltage rating and BIL specification influence the amount and type of dielectric material and its distribution in a transformer design. A complex voltage stress and impulse analysis is required for each transformer design to determine the exact voltage insulation requirements. Highvoltage transformers require a significant portion of their volume as insulation space, leading to increased size and capital cost.

The aforementioned design considerations and several other factors are of great importance in designing large high-performance power transformers. Many of these same design considerations also apply to superconductive transformers; however, not all of these considerations will have the same importance as they do to conventional transformers. Superconductive transformers will also have an additional set of constraints unique to their design.

\subsubsection{Superconductive Transformer Design}

Cryostable and ultrastable transformer designs are described in the following subsections.

\subsubsection{Cryostable Transformer Design}

Any time the current density in the superconductor, $\mathrm{J}$, exceeds the critical current density, $\mathrm{J}_{c}$, the superconductor will quench, losing its superconductivity. These excessive currents may be caused by faults, lightning, switching surges, inrush currents (when a major load or the transformer itself is first energized), or excessive load current. The high resistance of the normally conductive material would cause rapid heating and possibly catastrophic failure of the transformer unless a shunt path were provided to protect the windings until superconductivity could be restored. Such is the function of the stabilizer (Collings 1986).

Sufficient stabilizer is included in the cryostable conductor design so that resistive heat generated by the full-load current in the stabilizer during a global quench will be less than, or equal to, the conductor's ability to transfer heat to the refrigeration system. This is called "global" cryostability. This criterion guarantees that the conductor will not rise above $T_{c}$, the critical temperature of the superconductor. Thus, upon removal of the overload condition, the conductor should return to the superconductive state.

It would be prudent to include a safety margin in the conductor design so that superconductivity is maintained during moderate overcurrent condition. This practice should increase the reliability and 
usefulness of the transformer. A safety margin also will allow some load growth in excess of the transformer rating or simply reduce the number of global quenches that the windings would experience during routine operation.

If reduced amounts of stabilizer are used, the heat transfer capacity of the conductor may be sufficient to stabilize brief local disturbances but would not be sufficient to recover from a full global quench. Reduced amounts of stabilizer will reduce the conductor cost and eddy-current losses during operation, but they will also reduce the device reliability.

\subsubsection{Ultrastable Transformer Design}

As an alternative to a fully cryostable design, we also evaluated a stability philosophy we call an ultrastable design. The ultrastable design has a highly redundant superconductive material that eliminates the need for some or all of the stabilizer. This design is an approach to reducing high eddy-current losses in the stabilizer of cryostable designs. The ultrastable design philosophy represents a compromise of adding superconductive material and operating at a small percentage of $J_{c}$. This permits the ultrastable superconductive winding to remain superconductive while carrying any conceivable sustained or transient current overload condition.

Under the ultrastable design philosophy, either very little or no metal is in the conductor. As a result, a major power loss (due to eddy currents in the stabilizer) is avoided, and the size and cost of the transformer are potentially reduced. In place of stabilizer metal, a rather large safety margin (e.g., 20 times the rated current, depending on the application), is designed into the transformer conductors. The safety factor is chosen so that, under most conceivable circumstances, the winding current will not exceed the critical current and is a function of the power system characteristics where the transformer is installed. A typical value of 20 was chosen, which represents an approximate current at which instantaneous protective relays would be set. Thus, the probability of a global quench is not significantly different from zero. The assurance that the superconductor will rarely, if ever, quench, eliminates the requirement for stabilizer metal without sacrificing reliability. A passive current limiter could be built into the ultrastable transformer to protect against any extreme conditions that might exceed the design safety factors. If these protective measures prove effective, the safety factors could be reduced even further.

The issue of cryostable versus ultrastable superconductor is a consideration addressed when designing an optimal superconductive transformer. This and other issues discussed above were considered in developing an appropriate design methodology for comparing optimized conventional and superconductive transformer designs. To accommodate all these considerations and make appropriate comparisons, it was necessary to develop an appropriate computational methodology that incorporates these and any other pertinent considerations.

\subsection{Key Equations and Relationships}

A number of equations that compute various relationships and values are used in the transformer design model. Some of the more critical equations and specific methodologies used are discussed in this section. A more complete discussion of the approach is provided in Appendix B. 


\subsubsection{Core Expressions - Core Loss, Leakage Field, and Stray Loss Factor}

The core loss is measured as the watts dissipated per kilogram of magnetic material. For a given material, this loss at $60 \mathrm{~Hz}$ varies with the flux density. In our analysis, we considered two types of core material. Manufacturers' data for both 12-mil M-5 grain-oriented silicon steel and 2605S-2 alloy METGLAS steel were used for the conventional and amorphous cores, respectively.

The leakage field, along with the self-field, is part of the magnetic field to which the conductors are exposed, and will influence their losses. The leakage field is dependent primarily on the core material, the flux density at which the core is operating, and the transformer geometry. Equations describing core loss and field intensity were obtained from curve fits of manufacturers' data (Magnetics 1987, 1989).

As mentioned earlier, the stray loss is due primarily to inductive coupling of magnetic fields outside the transformer core with various magnetic materials such as the transformer support structure. Thus, the stray loss mechanism is similar to the core losses and is related to the transformer leakage field intensity. As the core flux density is increased, there is a nonlinear increase in the intensity of the field throughout the transformer. The increased magnetic field produces a corresponding rise in the stray losses within the transformer.

Stray losses may be estimated from the core flux density and core volume for a specific core material and transformer geometry. The quantity of noncore magnetic material is related to the core volume; e.g., larger cores will require more magnetic material for structural support and a larger transformer shell. Stray losses in transformer designs similar to those produced by our model are related to core flux density and core losses to obtain a stray loss factor.

The stray loss factor is an estimate of the transformer stray losses as a fraction of core losses at varying core flux densities. The iron loss equations used in our model for the two types of core material are

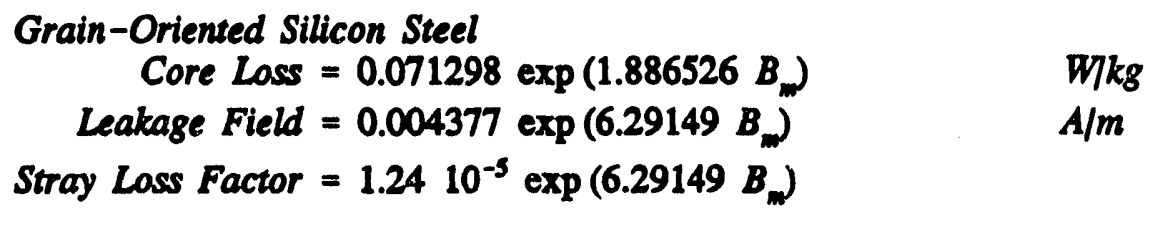

Amorphous Steel Alloy

$$
\text { Core Loss }=0.013818 \exp \left(1.997862 B_{m}\right) \quad W / k_{g}
$$$$
\text { Leakage Field }=0.2380 \exp \left(2.1563 B_{m}\right) \quad A / m
$$$$
\text { Stray Loss Factor }=\frac{F_{\text {Leaklam }}}{0.2955 \exp \left(-0.3965 B_{m}\right)}
$$ 
where

$$
\begin{aligned}
F_{\text {Landem }} & =1.2410^{-5} \exp \left(6.2915 \mathrm{~B}_{\mathrm{mlam}}\right) \\
\mathrm{B}_{\mathrm{mLam}} & =1.02105 \mathrm{H}_{\mathrm{o}}(0.095259) \\
\mathrm{B}_{\mathrm{m}} & =\text { the core flux density }(\mathrm{T}) \\
\mathrm{H}_{0} & =\text { the leakage field intensity (ampere-turns/meter). }
\end{aligned}
$$

Note: the degree of precision of the above equations does not reflect the quality of the data but rather the resolution of the curve fits to the data.

\subsubsection{Self-Field}

The magnetic field to which the conductor in the transformer winding is exposed consists of two components: leakage field, described above, and self-induced field, which is a function of the current in the conductor. The average field to which the conductors are exposed depends heavily on the transformer configuration, the winding geometry, the amount and placement of insulation, the arrangement of the conductors within the windings, the degree of stranding in the conductors, and other factors. Thus, field exposure of the windings on a microscopic level cannot be precisely calculated without a complex finite element analysis involving all of the above design factors for the myriad of potential transformer designs that must be evaluated.

We have not been able to locate a reasonably simple model for the distribution of self-field within the transformer. Hence, we theoretically bounded the problem. The actual field exposure of the windings is a design problem and cannot be precisely known until a transformer is built and tested. Thus, common transformer design practice generally involves providing a rough estimate of the average magnetic field within the windings, based upon rules-of-thumb and previous design experience. More detailed analysis is conducted, if necessary, only on the final optimized transformer design. If the effects of the self-field are found to be detrimental, it is possible to design transformers with interleaved windings or utilization of special conductors (such as Litz wire) to limit the magnitude of the self-field.

For our analysis, it was necessary to develop a simple model that reasonably approximates the average self-field to which the conductors in the transformer windings are exposed and that accounts for both macroscopic and microscopic field effects. The expression for the self-field used in both our conventional and superconductive transformer design models is given in Equation (3.11).

$$
H_{s}=\frac{0.7 I}{2 \sqrt{2} \pi R}\left(\frac{r_{s}}{R}\right)
$$

$$
\text { where } \begin{aligned}
\mathrm{H}_{1} & =\text { the self-induced magnetic field intensity } \\
\mathrm{I} & =\text { the conductor current } \\
\mathbf{r}_{1} & =\text { the strand radius } \\
\mathrm{R} & =\text { the conductor radius. }
\end{aligned}
$$

The above expression for the self-field was derived from Ampere's law for current flowing through a conductor, $\oint \mathrm{H} \cdot \mathrm{d} \ell=\mathrm{I}_{\text {encl }}$, and then modified to account for effects of field interaction between the strands that make up the conductors, among the conductors within the windings, and 
between the transformer windings themselves. Equation (3.11) provides an estimate of the average magnetic self-field induced by the current flowing in the transformer windings. The assumptions used in developing the above model are detailed below.

For a single cylindrical conductor in free space, the self-induced magnetic field is given by the following equation:

$$
H_{s}=\frac{I_{\text {ancl }}}{2 \pi r^{\prime}}(A / m)
$$

where $\mathbf{r}^{\prime}=$ radius from center of conductor

Thus, the average self-field within the body of a circular conductor is given by

$$
H_{s}=\frac{I}{2 \sqrt{2} \pi R}(A / m)
$$

Equations (3.12) and (3.13) describe the magnetic field intensity in and around a single wire, assuming uniform current density across the conductor cross section and assuming that there is no interaction with other sources of magnetic flux. Such a model might be used to determine the magnetic field intensity for an electric power transmission line or a similar application where the field-inducing conductor is relatively remote from other sources of magnetic field. These models are not accurate, however, in a transformer or other electrical device with wound conductors lying in proximity to one another, and which can have strong field interaction.

The self-field vectors in the area between nearby conductors with parallel current flow, such as within one winding of a transformer, lie in opposing directions and tend to cancel out one another. Figure 3.1a shows the conductors. Figure 3.1b shows a profile of the magnetic field intensity associated with the conductors. At some point directly between the centers of the wires, the field induced by each conductor is equal in magnitude and opposite in direction to precisely cancel with one another, as shown in the plot (assuming comparable conductor radii and current intensities).

As also shown in Figure 3.1b, the field magnitude within the conductors themselves rises monotonically from the center of each wire until reaching a maximum value at the conductor edges, and then rapidly decreases with distance from the wire. Outside these two conductors, the magnetic fields enhance one another, resembling at a distance a single wire with a higher current flow than that of either of the two single wires alone.

Conversely, two adjacent parallel conductors with equal current magnitudes, this time in opposite directions, enhance one another's self-induced magnetic fields between the centers of the wires, but cancel with one another elsewhere. Wires with opposing (antiparallel) current flow are sketched in Figure 3.2a. The induced magnetic field vectors between the centers of these two wires lie in the same direction and tend to support one another. As shown in Figure 3.2b, the self-field intensity in this area is thus increased beyond what it would have been with either conductor alone. 

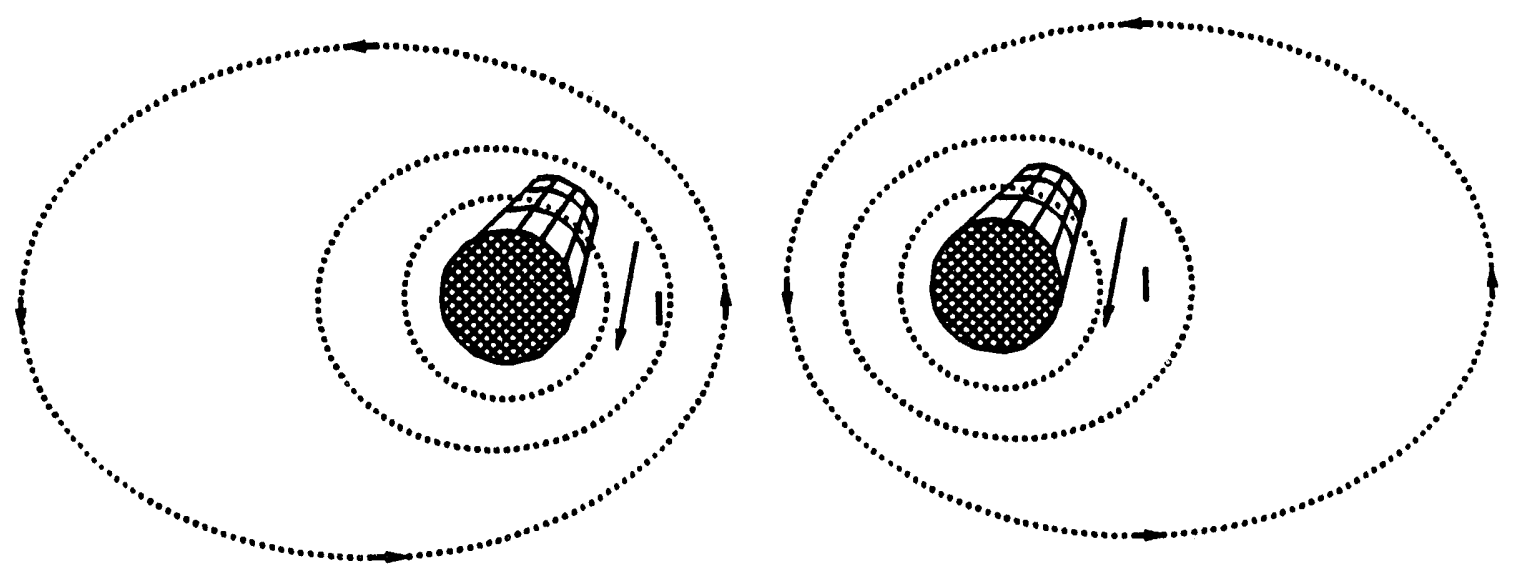

a) Magnetic Fields

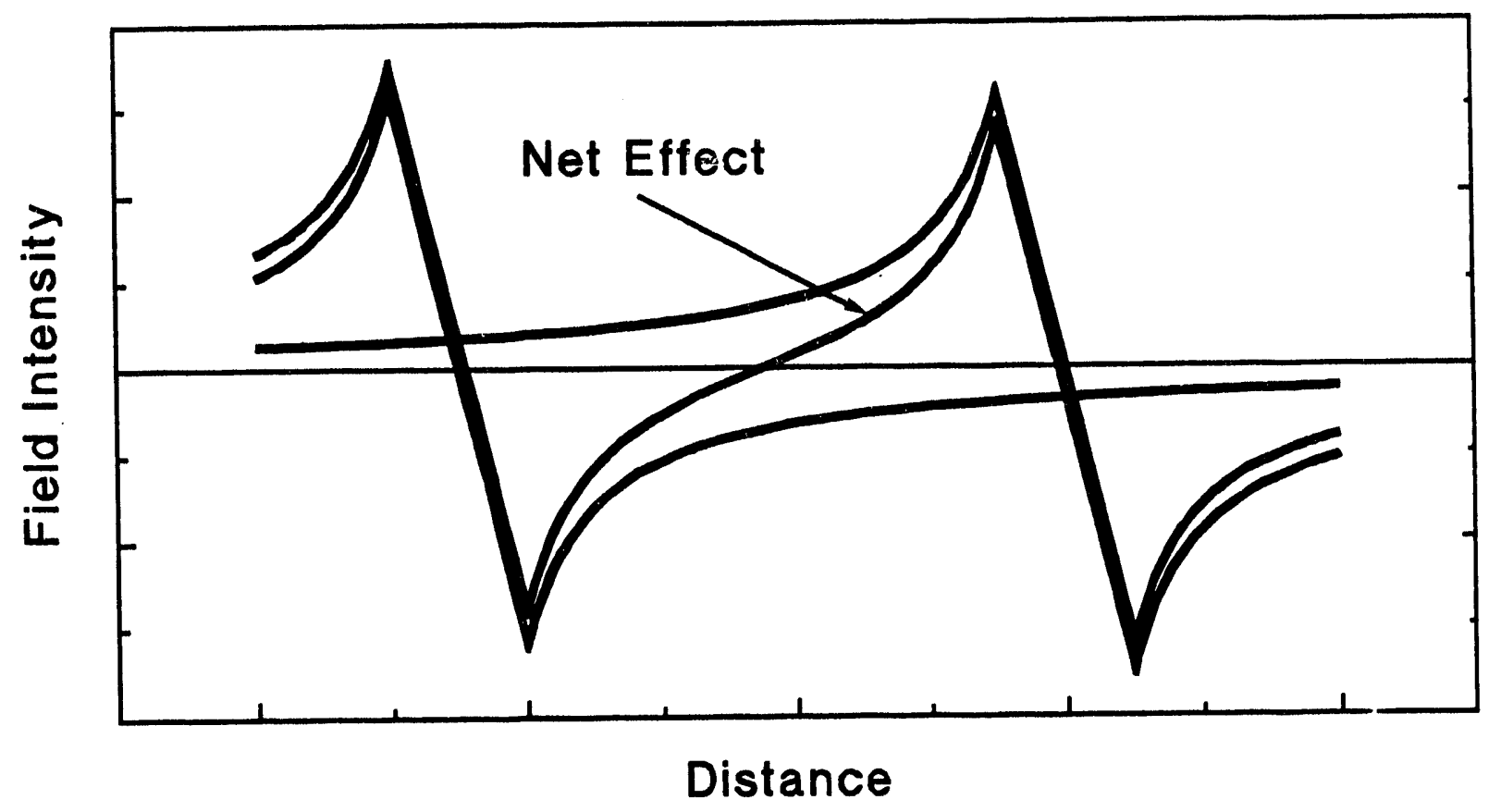

b) Magnetic Field Intensity Profile

Figure 3.1. Conductors with Parallel Currents 

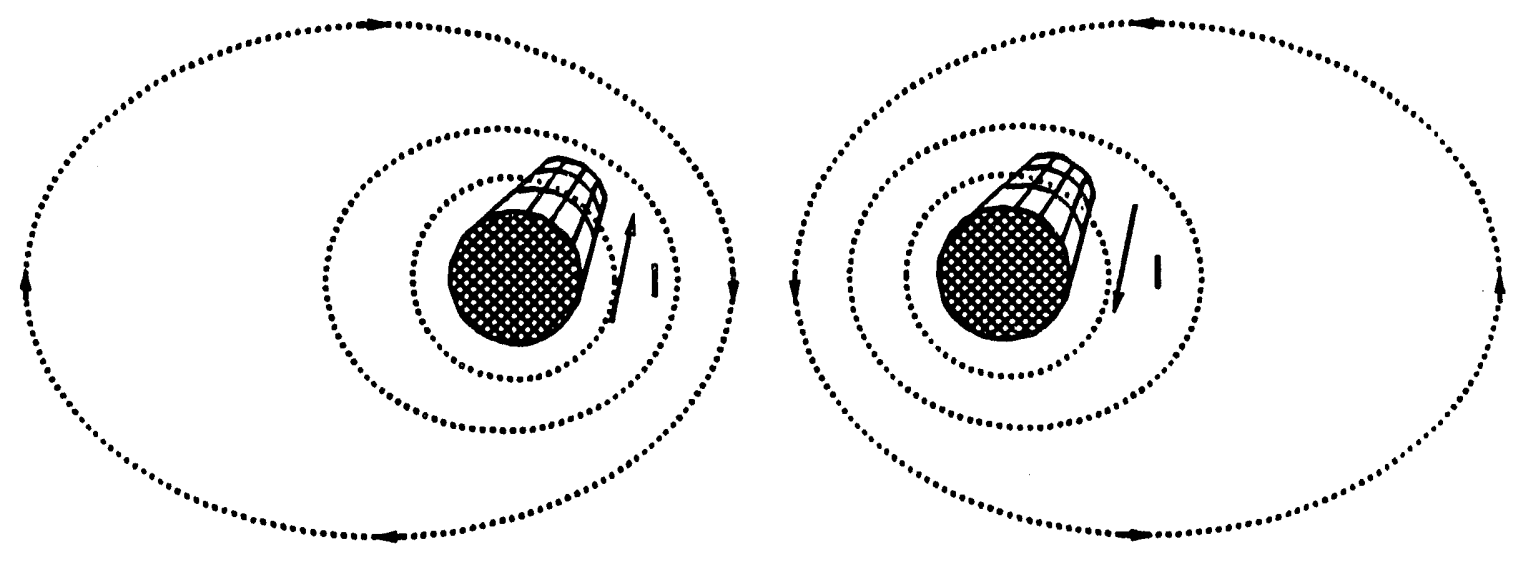

a) Magnetic Fields

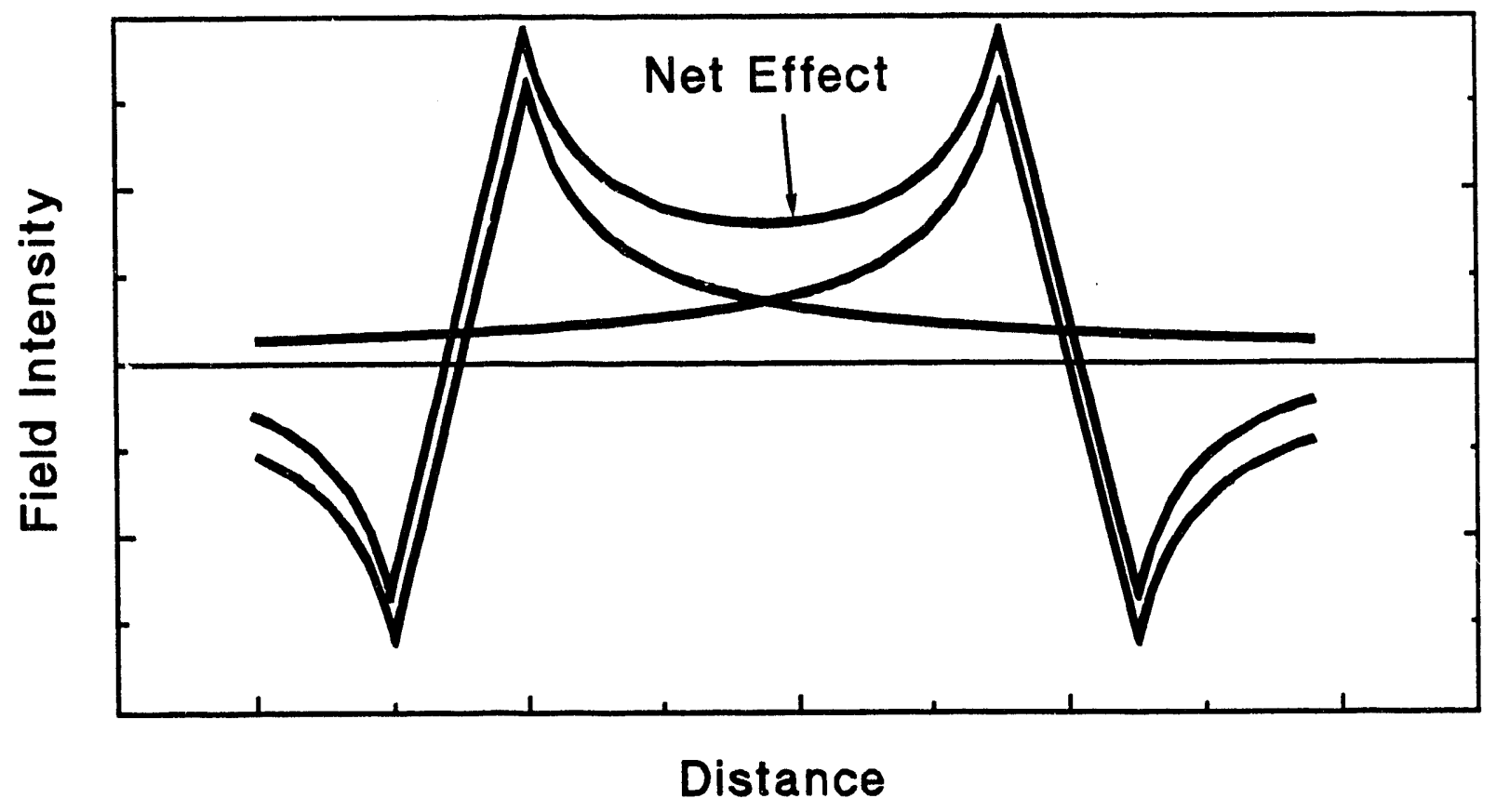

b) Magnetic Field Intensity Profile

Figure 3.2. Conductors with Anti-Parallel Currents 
Cancellation between the individual self-field components outside the two wires causes the net magnetic field intensity to be reduced, falling off rapidly toward zero with distance from the conductors.

The latter example of parallel conductors with opposing current flow approximates the macroscopic self-field behavior within the windings of a transformer. A typical winding configuration of a single-phase transformer with its associated (idealized) magnetic field profile is shown in Figure 3.3. Current flowing through the primary of the transformer induces a proportionate current flow in the secondary. The secondary current is induced in a direction that creates magnetic flux opposing that created by the primary winding. (This behavior was discussed more fully in Section 3.1, which described fundamentals of transformer operation.) The parallel currents within the primary and secondary windings flow in opposite directions as shown in Figure 3.3a. As a result, the field intensity exhibits a profile very similar to that of the two wires having opposite current flow discussed in the previous example. Figure $3.3 \mathrm{~b}$ reveals that the magnitude of the net self-induced magnetic field builds rapidly from the center of each winding, reaching its peak value at the periphery of the windings between the primary and secondary and falls off through the inter-winding insulation space. The self-field reaches somewhat smaller local maxima at the outer edges of the windings, where cancellation and distance from the windings causes it to decline rapidly.

The average self-field magnitude through the windings of the transformer is shown in Figure $3.3 \mathrm{~b}$. For the base case analysis, a value $70 \%$ of peak self-field intensity is a reasonable estimate for the average self-field intensity. When applied to Equation (3.13), this represents the constant scaling factor in Equation (3.11).

An additional modifying factor discussed below was added to the self-field model to account for the interaction of the individual self-field components of the strands within the conductors. The self-field induced by a perfectly packed, stranded conductor would behave as that of a uniform conductor of the same dimensions (neglecting strand insulation thickness). Obviously, stranded conductors are not perfectly packed: they contain free space because of the strand shapes, resulting from the twisting and braiding of the strands and from the insulation with which the strands are normally coated to reduce eddy-current losses. Thus, the geometry of the stranded conductor plays a significant role in the average self-field to which each strand is exposed. Filaments within superconducting wires were assumed to be densely packed and tightly twisted, such that their self-field interaction could reasonably be neglected.

The interaction between self-fields of any two strands within one of the conductors is shown in Figure 3.1. Self-fields are reduced between the centers of the strands and enhanced outside. Field interaction in multistranded conductors is somewhat more complex, but follows the same general principle. Self-field cancellation is nearly complete in strands near the center of the conductor, and is somewhat reduced toward the periphery. The net result is a reduction in the self-field of the stranded conductor over that of a similar uniform (nonstranded) conductor, which depends on the degree to which the conductor is divided. Thus, stranding the conductor tends to result in an average self-field reduction that varies as the ratio of the strand radius to the conductor radius. That is, $\mathrm{H}_{\mathrm{a}}$ is

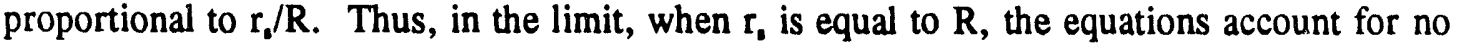
strand/conductor self-field canceling because the strand size equals the conductor size (i.e., there is only one strand per conductor). The inclusion of $r_{s} / R$ as a factor in our model [Equation (3.11)] completes the self-field equation used in our design study. Strand thickness within a conductor may 


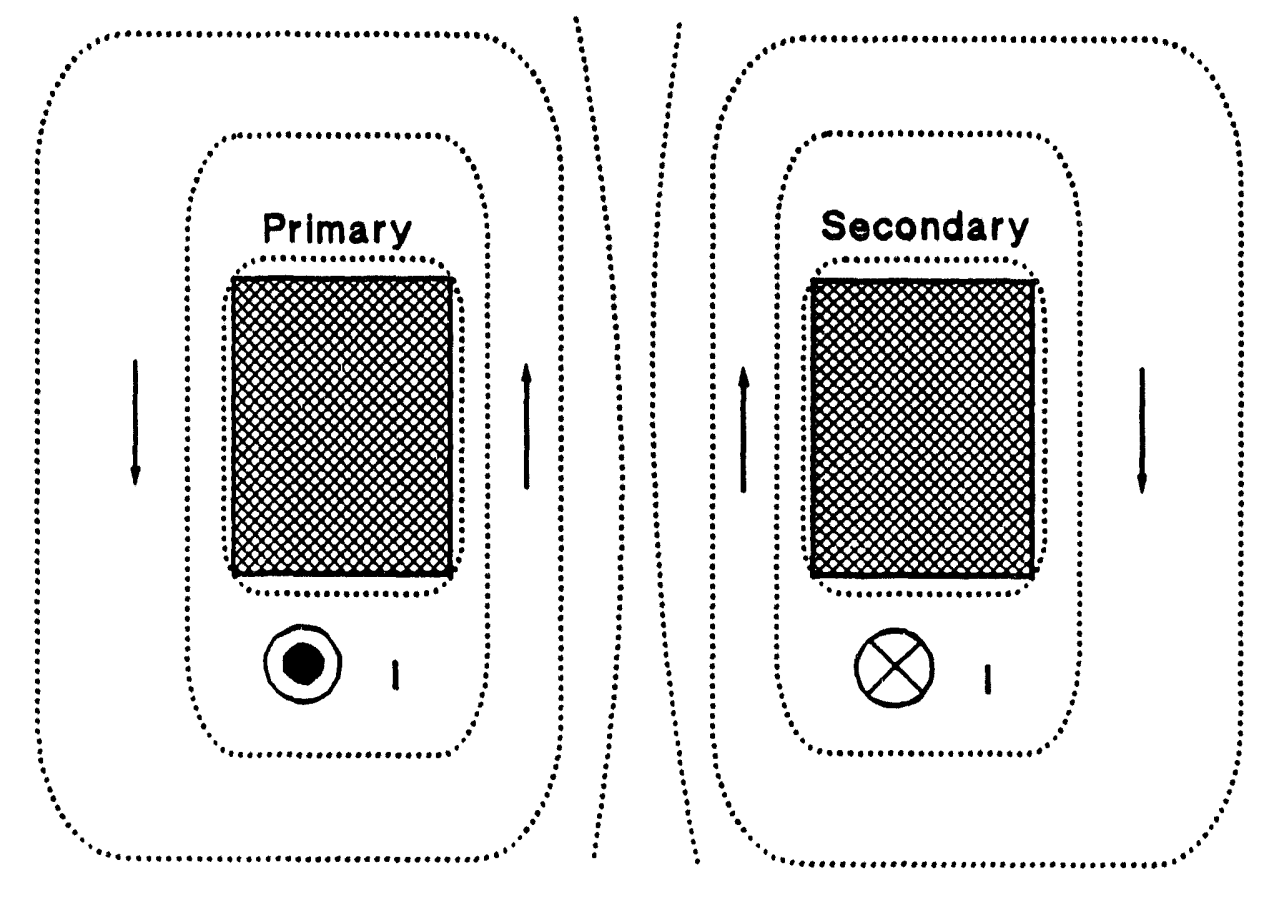

a) Magnetic Field

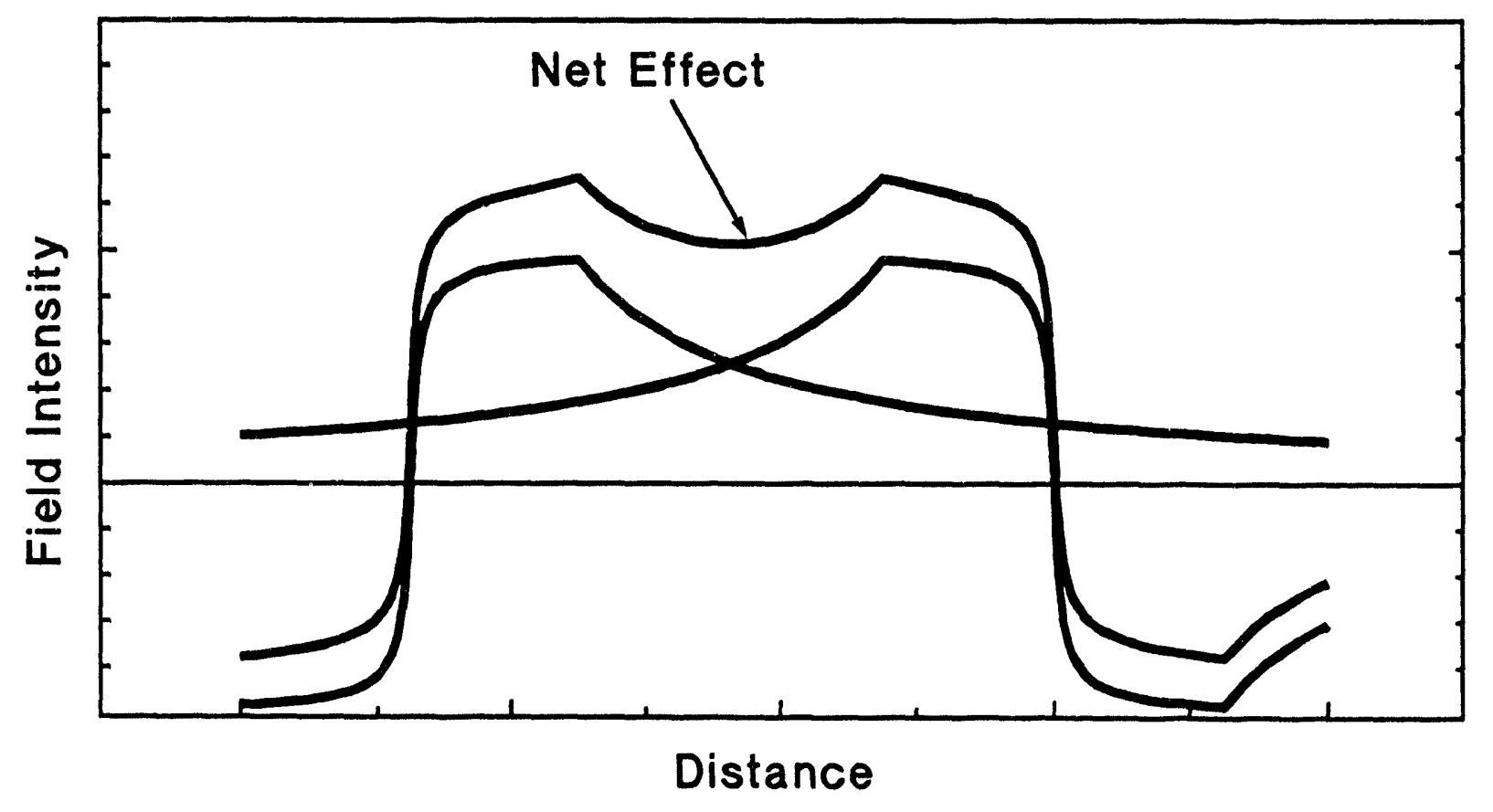

b) Idealized Magnetic Field Intensity Profile

Figure 3.3. Single-Phase Transformer 
thus be reduced to minimize losses resulting from the self-induced magnetic fields within the windings of the transformer. This allows the superconducting transformer analyst or designer an additional control variable in the design optimization process.

\subsubsection{Superconductor Cryostability Design Methodology}

The conductor models used in the fully cryostabilized superconducting transformer designs are simulated as having sufficient shunt copper to continuously carry full-rated current without heating beyond the critical temperature of the superconductor. Aluminum is also a potential stabilizer material. However, copper was chosen on the basis of its equivalent commercial availability (Collings 1986). The amount of stabilizer required to provide thermal stability for a specific application must be computed to ensure adequate system reliability.

The cryostability requirements are determined based on the Stekly cryostability criterion (Collings 1986). To achieve a cryostable conductor, the heat generated, due to I $R$ losses in the composite conductor while the superconductor is in the normal state (during a sustained global quench), must be less than or equal to the heat removed by the cooling medium (liquid nitrogen in the case of high-temperature superconductors). Thus, for a unit length of conductor, cryostability is ensured if the following relationship is savisfied:

$$
I^{2} \rho / A_{C u} \leq \dot{q} \pi D
$$

where $\quad I=$ the rated current of the conductor

$\rho=$ the electrical resistivity of the stability metal

$A_{C u}=$ the cross-sectional area of the copper stabilizer

$\dot{q}=$ the heat removal per unit surface area of the conductor

$D=$ the diameter of the composite superconductor cross section.

For a transformer of a given rating, the current density within the superconductor is fixed, so that the cross-sectional area of the superconducting component of the composite conductor is known. The total area of the composite conductor is simply the summation of the superconductor and stabilizer metal areas. The minimum diameter of the composite conductor required to ensure global cryostability may then be calculated. The relationship which must be solved is then:

$$
D^{3}-4 A_{S C} D / \pi-\frac{4_{\rho} I^{2}}{\dot{q} \pi^{2}}=0
$$

where $\mathrm{A}_{\mathrm{SC}}=$ the cross-sectional area of the superconductor component of the composite conductor.

The cross-sectional area of the superconductor is simply the full-load phase current divided by the critical current density (RMS) and by the operating margin (the ratio of the rated current density to critical current density). The cubic equation is solved using Newion's method to yield the diameter of the composite conductor at the point of marginal cryostability. Only the right-most positive real root on the $x-y$ plane provides a valid solution for the conductor diameter. The total conductor 
cross-sectional area, the copper cross-sectional area of the copper stability metal, and the fractions of superconductor and copper in the total conductor may then be determined.

The heat transfer rate, $q$, calculated to be $20 \mathrm{~W} / \mathrm{cm}^{2}$, was based upon the heat flux from the bottom surface of a horizontal polished platinum plate immersed in liquid nitrogen, and represents the minimum that might be expected (Lyon 1968).

The above methodology does not include thermal conductivity of the wire, or the ability to transfer heat from inside the conductor to the cooling medium (at the surface of the conductor). Not included in the model because of the difficulty associated with different conductor design configurations, the operating margin and conservative estimate of the surface heat transfer rate (and such factors as thermal inertia) compensate for the internal thermal conductivity.

An operating margin was also included that would allow the transformer to operate continuously as much as $40 \%$ above its rating without loss of superconductivity. This assumption is in accordance with the generally conservative design requirements for electrical components used in electric power transmission and distribution systems, designed to have a high degree of reliability.

\subsubsection{Superconductor Hysteresis Losses}

A number of models exist that predict hysteresis losses within superconducting filaments. Each of these models provides a reasonable estimate of the ac loss within specific types of superconducting material and for limited ranges of magnetic field intensities, frequencies, and conductor geometries. An equation that accurately models hysteresis loss for one superconductor material or application will not necessarily be valid for another. At the time of this analysis, we could find no published validated model for accurately predicting hysteresis losses within high-temperature ceramic superconductors. We have therefore chosen to use a model developed for low-temperature niobium-based superconductors by W. J. Carr of Westinghouse Research Laboratories (Carr 1977). The hysteresis loss in the superconductive material is found by the following equation:

$$
\text { Hysteresis loss }=\frac{V o l}{\ln \lambda B_{L} d f J_{c}}-\text { watts }
$$

where Vol $=$ total conductor volume $\left(\mathrm{m}^{3}\right)$

$\lambda=$ fraction of superconductor in conductor

$B_{L}=$ average winding magnetic flux density (both leakage and self-magnetic fields) (T)

$\mathrm{d}=$ superconductor filament diameter (m)

$f=$ frequency $(\mathbf{H z})$

$\mathrm{J}_{\mathrm{c}}=$ superconductor critical current density $\left(A / \mathrm{m}^{2}\right)$. 
We assume that this model provides a reasonable estimate of ac losses in future ceramic superconductor materials, once fabrication difficulties have been solved, to minimize losses caused by the granular structure of the ceramic materials, and to increase critical current densities to more useful levels.

\subsubsection{Eddy-Current Losses}

Winding eddy-current losses in a composite superconductor are greatly dependent on the conductor geometry. The relative position of the superconducting filaments within the composite strand and the degree with which these filaments are twisted, influence the degree to which external ac magnetic fields penetrate the composite strand to where significant eddy currents may be induced. The eddy-current loss in the superconductive windings as determined by Carr (1977) is:

$$
\text { Eddy current loss }=\text { CuVol }\left(f B_{L} L_{p}\right)^{2}\left[\frac{1}{2 p_{c u}}\right]\left[1+\left(\frac{\pi r_{p}}{L_{p}}\right)^{2}\right] \text { watts }
$$

where $\mathrm{CuVol}=$ total copper volume in the transformer windings $\left(\mathrm{m}^{3}\right)$

$\mathrm{f}=$ frequency $(\mathrm{Hz})$

$B_{L}=$ average winding magnetic flux density (both leakage and self-magnetic fields) (T)

$\mathrm{L}_{\mathrm{p}}=$ filament twist pitch $(\mathrm{m})$

$r_{1}=$ superconductor-copper matrix strand radius $(m)$

$\rho_{o u}=$ resistivity of copper (at the proper temperature) $(\Omega-m)$.

\subsubsection{Dielectric Loss}

The dielectric loss is caused by an oscillatory voltage gradient within the conductor insulating material. The time-varying electric field induces molecules within the dielectric insulation material to align themselves in response. The oscillatory force required to realign the dipoles at the operating frequency results in power dissipation. Thus, the dielectric loss is analogous to the iron hysteresis loss mechanism in iron due to time-varying magnetic fields. The dielectric loss term is relatively small (as opposed to the core hysteresis loss) for both conventional and superconductive designs, but may possibly be a relatively significant load on the cooling system in the cryogenic environment of the superconducting transformer. The equation used to compute dielectric losses was taken from a Westinghouse transformer design study (Westinghouse Electric Corporation 1982). The dielectric loss expression used is

$$
\text { Dielectric loss }=\epsilon_{r} \epsilon_{0} 2 \pi f E^{2} \tan (8) V o l_{\text {Ins }} \phi
$$

where $\quad \epsilon_{\mathrm{r}}=$ relative permittivity

$\epsilon_{0}=$ permittivity of free space

$\mathrm{f}=$ frequency $(\mathrm{Hz})$

$E=$ voltage stress on insulation $(\mathrm{V} / \mathrm{m})$

$\tan (\delta)=$ mean loss tangent of insulation 


$$
\begin{aligned}
\mathrm{Vol}_{\mathrm{Ine}} & =\text { insulation volume (calculated by the program) }\left(\mathrm{m}^{3}\right) \\
\phi & =\text { number of transformer phases (single- or three-phase). }
\end{aligned}
$$

\subsubsection{Heat Leak Calculations}

Because the superconducting transformer windings operate significantly below ambient temperature, thermal influx into the cryostat must be accounted for and removed by the refrigeration system. Although not directly a part of the transformer losses, the power consumed by the refrigeration system to remove the heat influx into the cryostat must be included in the total transformer losses.

The thermal heat leak is composed of two components: radiative heat transfer and conductive heat transfer. The equations used to estimate these are

$$
\text { Radiant heat leak }=\dot{q},(\text { Cryostat surface area })
$$

$$
\text { Conductive heat leak }=\frac{7.1}{50 \sqrt{\Phi}} M V A\left(T_{\text {amb }}-T_{c r y o}\right)\left(\frac{N B_{1}}{k V_{1}}+\frac{N B_{2}}{k V_{2}}\right)
$$

where

$$
\begin{aligned}
\dot{q}_{r} & =\text { radiant heat influx, } 18 \mathrm{~W} / \mathrm{m}^{2} \text { (vacuum insulation) } \\
\phi & =\text { number of transformer phases (single- or three-phase) } \\
\text { MVA } & =\text { transformer power rating (MVA) } \\
\mathrm{T}_{\text {amb }} & =\text { ambient temperature }(\mathrm{K}) \\
\mathrm{T}_{\text {ryyo }} & =\text { cryostat temperature }(\mathrm{K}) \\
\mathrm{NB}_{1} & =\text { number of primary bushings } \\
\mathrm{NB}_{2} & =\text { number of secondary bushings } \\
\mathbf{k V}_{1} & =\text { voltage of primary windings }(\mathrm{kV}, \mathrm{rms}) \\
\mathbf{k V}_{2} & =\text { voltage of secondary windings }(\mathrm{kV}, \mathrm{rms})
\end{aligned}
$$

The radiative heat leak is the heat that passes through the vacuum insulation space between the cryostat and the ambient-temperature transformer tank. As the core is assumed to be outside the cryostat, some heat generated by core losses will also be absorbed in the cryostat. The conductive heat leak is due primarily to heat passing through the transformer bushings; its magnitude is mainly a function of the bushing geometry (and the number of bushings) in a given transformer design. For the cases considered, the conductive heat leak was typically found to be much greater than the radiative heat leak term.

\subsubsection{Refrigeration System Coefficient of Performance}

The refrigeration system COP is a measure of the efficiency with which the cryo-cooling unit can remove heat from the cryostat. The COP is equal to the energy removed divided by the electrical refrigeration load. The COP is determined by four factols:

- the operating temperature of the cryostat

- the ambient temperature where waste heat is rejected 
- the amount of heat processed by the refrigerator

- the technology used in the refrigeration system.

For our study, the base case operating temperature of the cryostat is $77 \mathrm{~K}$, which is the boiling point of liquid nitrogen at standard atmospheric pressure. This temperature was chosen to enable the use of liquid nitrogen, which is among the cheapest cryogenic fluids commercially available. It boils at a temperature below the predicted operating temperature range of future high-temperature ceramic superconductors.

The ambient temperature assumed for the base case is $300 \mathrm{~K}\left(26.85^{\circ} \mathrm{C}, 80.33^{\circ} \mathrm{F}\right)$. The ambient temperature will undoubtedly change because of daily and seasonal climatic changes. However, $300 \mathrm{~K}$ is a fairly conservative representation of the expected average ambient temperature. At any given time, the power consumed by the refrigeration system will depend on the ambient temperature, with higher temperatures leading to more power consumption. Therefore, during a cold winter day, the power consumed by the refrigeration system will be much less than for a hot summer day. The refrigeration system must continue operation for all environmental conditions, so a $40 \%$ capacity margin was added to ensure that the equipment would be sized to handle the worst-case situation rather than the seasonal average power.

Refrigeration systems have economies of scale that enable more efficient operation with larger systems (Strobridge 1974). The thermal efficiency (COP) of the cryo-cooling unit tends to increase with size, i.e., with increasing cooling capacity. Between 10 and 20 watts of electricity are required to remove each thermal watt for a typical modern cryopump operating at liquid nitrogen temperature. The expression used to determine the COP in our model was based on a best fit to the Strobridge data, which relates the amount of cooling capacity required for a given application to the COP of the cryogenic refrigeration system.

$$
\begin{aligned}
& C O P=0.055515+0.008786 \text { in (Heat removed) } \\
& \text { Heat removed }=\text { heat generated in cryostat }(k W t h)
\end{aligned}
$$

The COP is dimensionless, but is equal to

\section{Energy removed from cryostat (watts) \\ Electrical refrigerator load (watts)}

The actual determination of the COP, in which the operating and ambient temperatures were assumed and a baseline process defined, depends on the cooling capacity of the cryo-cooler.

The thermal COP also depends on the type of cycle used, with actual process efficiency less than the ideal Carnot cycle. The technology used was not directly specified in this project, but provides a conservative estimate of current state-of-the-art performance. It is expected that a Stirling cycle refrigerator will eventually be used for superconductive transformers, with the technology meeting or exceeding the COP estimates used in this study. 


\subsection{Base Case Design Parameters}

Literally thousands of different transformer designs might warrant investigation. The task of assessing possible economic benefits can be performed more easily if a limited number of cases are selected that represent most of the transformer designs typically encountered in the utility power system. Thus, the base case transformer designs have a twofold requirement:

- They must be indicative of the types of transformers that are likely to be used in the future so that a reasonable comparison between conventional and superconducting designs can be made.

- They must correspond to the most common sizes and voltages found in the electric utility system so that the task of assessing market penetration and benefits to the nation has adequate input.

It is also necessary to define base case material parameters for high-temperature superconductors, to form a relative basis of comparison in the evaluation of superconductive designs.

Therefore, we have defined base case transformer designs, as well as base case materials specifications. The sensitivity analyses reveal relative impacts of changing these designs or specifications. This allows the economic and market penetration analyses to be performed with as small a set of data as possible, yet maintains a high degree of accuracy in assessing the relative contributions superconductivity may yield to power transformer design.

\subsubsection{Base Case Design Selections}

Because the possible benefits of superconductivity applied to transformers is likely to be greater for larger power transformers than for smaller distribution transformers, only large power transformers connected to the transmission system were considered in this study. Table 3.1 shows the transformer base case design selections. These selections represent all of the major classifications of large power transformers in the power system today. The voltage levels and power ratings are representative of the transformers currently used in each application and reflect trends that may be applicable for future applications.

Step-up transformers receive their input from individual generators and transform that energy to voltage levels for bulk energy transmission. Baseload and intermediate load generators are typically located some distance from load centers and, thus, it is most economic to transmit energy at high voltages (500 kV for our analyses). Peaking plants are typically smaller and located closer to the load centers; hence, the more economic choice is to transmit energy at $230 \mathrm{kV}$. The $500-\mathrm{kV}$ and $230-\mathrm{kV}$ values were selected as representative by examining a map of the New England Power Pool (NEPOOL) grid and by MVA sales of transformers recorded by the National Electric Manufacturers Association (NEMA 1986). We selected $115 \mathrm{kV}$ as the minimum transmission voltage of interest (this voltage is commonly used for subtransmission connections to $500-\mathrm{kV} / 230-\mathrm{kV}$ grids).

Step-up generator sizes to be included in the analysis were limited to what we feel is most representative of the market that will exist in the next century. The current trend is toward smaller, more quickly constructed and utilized plants. Thus, sizes near the midpoint (or smaller) for each application were used. 
Each design in Table 3.1 is analyzed with standard and amorphous cores, and with conventional technology or superconductive technology using either cryostable or ultrastable design philosophies. Thus, for each line of Table 3.1, there are 6 base case designs, for a total of 36 base case designs.

Table 3.1. Transformer Base Case Design Selections

\begin{tabular}{|c|c|}
\hline $\begin{array}{l}\text { Size, } \\
\text { MVA }^{(\omega)}\end{array}$ & $\begin{array}{c}\text { Input } \\
\text { Voltage. KV(o) }\end{array}$ \\
\hline
\end{tabular}

$\begin{array}{lrcc}\text { Step-up (baseload) } & 630 & 22 & 500 \\ \text { Step-up (intermediate load) } & 315 & 22 & 500 \\ \text { Step-up (peaking) } & 63 & 13.8 & 230 \\ \begin{array}{l}\text { Autotransformer } \\ \text { (high-voltage transmission) }\end{array} & 1000 & 500 & 230 \\ \begin{array}{l}\text { Autotransformer } \\ \text { (subtransmission voltage) }\end{array} & 200 & 230 & 115 \\ \begin{array}{l}\text { Step-down } \\ \text { (transmission/distribution } \\ \text { interface) }\end{array} & 30 & 115 & 34.5 \\ \end{array}$

(a) MVA sizes for step-up units are determined from generator unit sizes in MW (EPRI 1986). To account for the difference in the generator MW rating and the transformer MVA rating, MW is multiplied by 1.259 (NEMA 1986). Peaking platits have unit sizes ranging from 50 to $100 \mathrm{MW}$ (at central stations) and 5 to $50 \mathrm{MW}$ (when dispersed). A nominal value of $50 \mathrm{MW}$ was selected, so a transformer rating of 63 MVA was used. MVA sizes for autotransformers are based on average bank size to be installed between 1985 and 1989 (NEMA 1986).

(b) Input voltages for step-up transformers (generator output voltages) are from ANSI Standard C84.1 and documents from actual power plants. Input voltages for autotransformers were determined by their location in the grid. The input voltage for the step-down transformer was determined by the lowest voltage in the simplified model of the grid $(115 \mathrm{kV})$.

(c) Output voltages from step-up transformers are $500 \mathrm{kV}$ for baseload and intermediate load generators (located some distance from the load) and $230 \mathrm{kV}$ for peaking (located closer to the load). These voltages were selected based on MVA sales of autotransformers (NEMA 1986), NEPOOL maps, and a need to have a simplified model of the entire grid. Output voltages for autotransformers were determined by their location in the grid. The output voltage for the step-down transformers used in this study is $34.5 \mathrm{kV}$ because this is the trend in distribution system voltages. 


\subsubsection{Base Case Superconductive Material Specifications}

The material specifications were selected primarily from typical parameters of low-temperature superconductors. There is currently little information abcut the configuration and performance of the high-temperature ceramic superconductor. However, information on low-temperature superconductive technology is available; this information is assumed to be representative of high-temperature superconductors. Of course, these parameters are varied in the sensitivity analysis to determine the exact $e^{\prime}$.ect of these assumptions on the overall design and viability of the transformer. The base case sulections merely offer a basis of comparison for the sensitivity analysis.

The superconductor configuration assumed for the transformer windings follows from previous designs postulated for low-temperature superconductor ac applications. It consists of multifilamentary superconductive filaments in a matrix of copper wire. Although ceramic high-temperature superconductive conductors may not follow this exact design philosophy (thin-film tape conductors may be used to form the transformer windings), many of the same loss mechanisms might carry over from experience with low-temperature superconductors. This provides a basis of comparison to possible conductor designs that may be in future transformer designs.

The materials specifications that apply to superconductive transformer design are the critical current density, $J_{c}$; the filament diameter, $d$; the strand radius, $r_{c}$; and the twist pitch, $L_{p}$. (The twist pitch is the length of conductor in which the spirally wound filaments are completely transposed).

The $\mathrm{J}_{\mathrm{c}}$ is a very important parameter because it directly determines the amount of superconductive material required; the base case assumption for $J_{c}$ is $10^{5} \mathrm{~A} / \mathrm{cm}^{2}$. Critical current density, as defined in this study, assumes that the conductor can carry the specified current density without quenching, while operating at $77 \mathrm{~K}$ in a field comparable to the magnetic environment of transformer windings. This current density has not yet been achieved in bulk samples of ceramic superconductors, but is expected to be achieved if material fabrication research and development continues. This $\mathrm{J}_{\mathrm{c}}$ can be easily obtained in low-temperature superconductive transformer designs.

The filament diameter, strand radius, and the twist pitch have no comparative basis because ceramic superconductor development is in its early stages (with no suitable conductors fabricated). Therefore, these parameters have been taken directly from low-temperature conductor designs used in ac applications. The materials specifications are given in Table 3.2.

\subsection{Base Case Results}

As discussed in Section 3.2 the major transformer losses are modeled in the transformer design and performance code. Table 3.3 shows the types of losses that the code considers in the design and performance calculations.

The cooling system of the superconducting transformer requires input of electrical energy to remove heat generated by losses within the cryostat and heat entering the cryostat from the environment. Heat that leaks into the cryostat through the vacuum vessel walls and through the 
Table 3.2. Base Case Material Specifications

\begin{tabular}{lcc}
\multicolumn{1}{c}{ HTS Material Parameter } & Base Case Value \\
\cline { 1 - 1 } Critical current density $\left(J_{0}\right)$ & & $10^{5} \mathrm{~A} / \mathrm{cm}^{2(a)}$ \\
Strand radius $\left(r_{a}\right)$ & $500 \mu \mathrm{m}$ \\
Filament diameter $(D)$ & $50 \mu \mathrm{m}$ \\
Twist pitch $\left(\mathrm{L}_{\mathrm{p}}\right)$ & $2 \mathrm{~cm}$
\end{tabular}

(a) At $77 \mathrm{~K}$ and maximum magnetic field intensity in transformer windings.

Table 3.3. Conventional and Superconducting Transformer Losses

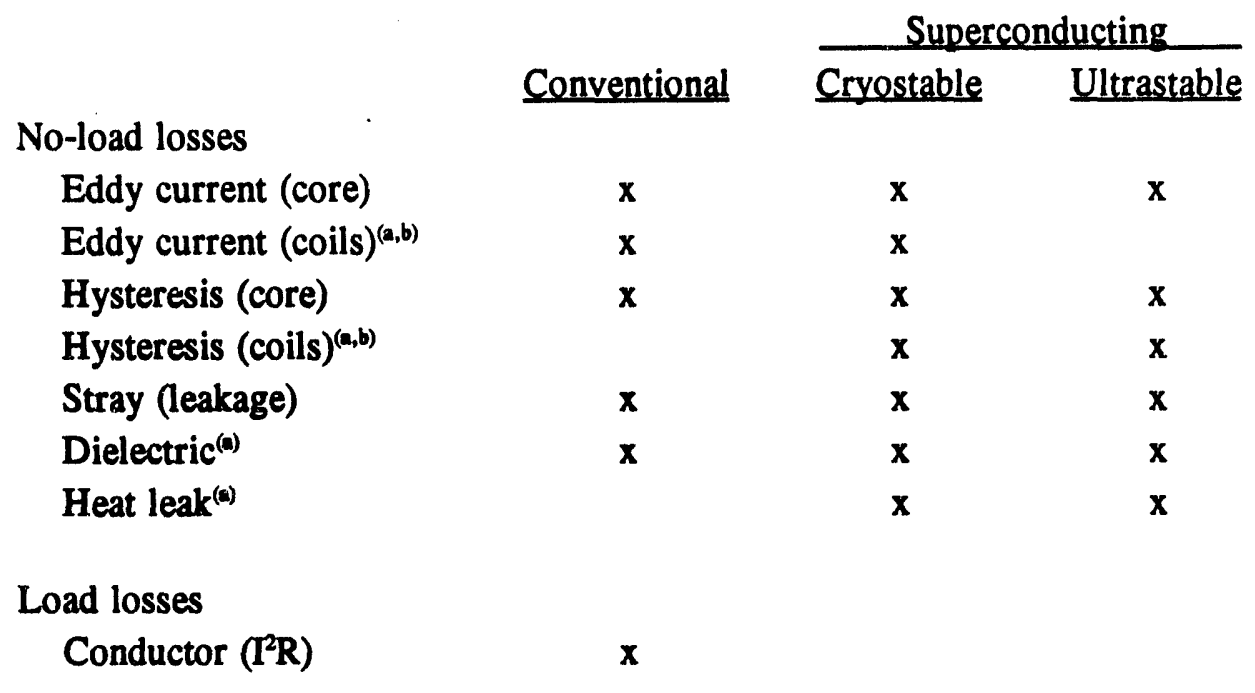

(a) Removed by cryocooler in superconducting designs.

(b) Self-field influence calculated at design current.

conductor terminal bushings is not an electrical loss but does represent an additional load on the cooling system. Thus, the cryocooler COP effectively becomes an additional loss term for the superconducting transformer.

Of the losses listed in Table 3.3, those that must be removed by the cryocooling system have a disproportionate effect or. the transformer efficiency. These losses are hysteresis losses in the superconductor, eddy-current losses in the cryostability metal, dielectric losses in the insulation material, and heat leak into the cryostat. All are scaled by the COP of performance of the cooling system. 


\subsubsection{Base Case Conventional Transformer Design and Performance Results}

Tables 3.4 through 3.18 present the full-load performance and design information on the minimum life-cycle cost designs for each base case transformer. Included in each table are the fullload efficiency; all the modeled losses; the percentage of the total thermal loss that each of the losses represents (shown in parentheses below the loss); the percentage of the total electrical loss represented by each loss [shown in square brackets]; and the COP of the cryocooler. Following each table is a discussion of the pertinent results.

Table 3.4 shows conventional grid transformer performance for conventional core transformers. As expected, and as seen in practice, the full-load efficiency of the conventional transformer increases with capacity. The dominant loss for conventional autotransformers is the $I^{2} R$ conductor loss (load loss), which represents approximately $70 \%$ of the total losses. The losses shown are full-load losses; in actual practice, the transformer operates at less than full load much of the time. Thus, the breakdown of losses on an annual basis will be considerably different. For the baseline assumptions used to evaluate autotransformers, the annual load losses are approximately $42 \%$ of the peak losses shown in these tables. Therefore, the annual load loss represents about $52 \%$ of the total annual losses. This is further explained in Appendix C.

The other major losses for conventional autotransformers are core and leakage losses (no-load losses). These losses result from eddy currents and hysteresis in the core and other noncoil metal caused by the changing magnetic field. Load losses, core losses, and leakage loss combined account for about $99.9 \%$ of the full-load and annual losses in a conventional transformer. For conventional core autotransformers, total losses are evenly split between annual load and no-load losses.

Transformers with amorphous cores offer the potential for greatly reduced core losses by increasing the resistivity of the core material. This increased resistivity reduces eddy currents in the core, and the amorphous structure greatly reduces hysteresis losses. Because of the reduced losses, higher efficiency amorphous core transformer show much promise for. the future. However, the increase in efficiency comes at the cost of lower possible flux densities and a lower stacking factor (percentage of the core cross-sectional area that is actually magnetic material) than conventional silicon steel cores. Both these factors tend to push the design toward larger, more expensive cores and coils.

Table 3.5 shows the full-load performance of the minimum life-cycle cost designs for conventional autotransformers with amorphous cores. As anticipated, the core losses have been greatly reduced; core losses for the amorphous core design are roughly one-quarter the conventional core losses for the designs shown in Table 3.4. Leakage losses have also been dramatically reduced in these designs.

As a result of the design optimization, the conductor loss of the smaller designs has increased slightly to partially offset the decreased core losses. The other transformer losses remain an insignificant part of the total losses. Overall, amorphous core increased the full-load efficiency of the autotransformers by 0.02 to $0.05 \%$. 
Table 3;4. Conventional Grid Transformer Performance; Conventional Core

\begin{tabular}{|c|c|c|c|}
\hline & & insformer $\mathrm{R}$ & \\
\hline & $30 \mathrm{MVA}$ & 200 MVA & $1000 \mathrm{MVA}$ \\
\hline Efficiency (\%) & 99.738 & 99.906 & 99.939 \\
\hline Core loss (W) & $\begin{array}{c}20,030 \\
(25.43)^{(0)} \\
{[25.43]^{(0)}}\end{array}$ & $\begin{array}{l}47,790 \\
(25.37) \\
{[25.37]}\end{array}$ & $\begin{array}{l}174,300 \\
(28.66) \\
{[28.66]}\end{array}$ \\
\hline Conductor loss $(\mathrm{W})$ & $\begin{array}{l}55,540 \\
(70.52) \\
{[70.52]}\end{array}$ & $\begin{array}{l}133,000 \\
(70.59) \\
{[70.59]}\end{array}$ & $\begin{array}{c}413,400 \\
(67.98) \\
{[67.98]}\end{array}$ \\
\hline Cryo heat leak (W) & $\begin{array}{c}0 \\
(0.00) \\
{[0.00]}\end{array}$ & $\begin{array}{c}0 \\
(0.00) \\
{[0.00]}\end{array}$ & $\begin{array}{c}0 \\
(0.00) \\
{[0.00]}\end{array}$ \\
\hline Hysteresis coil (W) & $\begin{array}{c}0 \\
(0.00) \\
{[0.00]}\end{array}$ & $\begin{array}{c}0 \\
(0.00) \\
{[0.00]}\end{array}$ & $\begin{array}{c}0 \\
(0.00) \\
{[0.00]}\end{array}$ \\
\hline Eddy-current coil (W) & $\begin{array}{l}70.23 \\
(0.09) \\
{[0.09]}\end{array}$ & $\begin{array}{l}179.6 \\
(0.10) \\
{[0.10]}\end{array}$ & $\begin{array}{l}555.0 \\
(0.09) \\
{[0.09]}\end{array}$ \\
\hline Leakage loss (W) & $\begin{array}{l}3,116 \\
(3.96) \\
{[3.96]}\end{array}$ & $\begin{array}{l}7,435 \\
(3.95) \\
{[3.95]}\end{array}$ & $\begin{array}{l}19,800 \\
(3.26) \\
{[3.26]}\end{array}$ \\
\hline Dielectric loss (W) & $\begin{array}{l}1.597 \\
(0.00) \\
{[0.00]}\end{array}$ & $\begin{array}{l}2.929 \\
(0.00) \\
{[0.00]}\end{array}$ & $\begin{array}{l}15.81 \\
(0.00) \\
{[0.00]}\end{array}$ \\
\hline Total thermal loss (W) & 78,760 & 188,400 & 608,100 \\
\hline Total electrical loss (W) & 78,760 & 188,400 & 608,100 \\
\hline Cryocooler COP & NA & NA & NA \\
\hline
\end{tabular}

(a) Percentage of total thermal losses shown in parentheses ( ).

(b) Percentage of total electric losses shown in square brackets [ ]. 
Table 3.5. Conventional Grid Transformer Performance; Amorphous Core

\begin{tabular}{|c|c|c|c|}
\hline & \multicolumn{3}{|c|}{ Transformer Rating } \\
\hline & $30 \mathrm{MVA}$ & $200 \mathrm{MVA}$ & $1000 \mathrm{MVA}$ \\
\hline Efficiency (\%) & 99.786 & 99.926 & 99.954 \\
\hline Core loss (W) & $\begin{array}{c}4,364 \\
(6.78)^{(0)} \\
{[6.78]^{(0)}}\end{array}$ & $\begin{array}{l}10,800 \\
(7.28) \\
{[7.28]}\end{array}$ & $\begin{array}{l}44,160 \\
(9.62) \\
{[9.62]}\end{array}$ \\
\hline Conductor loss (W) & $\begin{array}{l}59,390 \\
(92.25) \\
{[92.25]}\end{array}$ & $\begin{array}{l}135,900 \\
(91.64) \\
{[91.64]}\end{array}$ & $\begin{array}{c}408,700 \\
(89.02) \\
{[89.02]}\end{array}$ \\
\hline Cryo heat leak (W) & $\begin{array}{c}0 \\
(0.00) \\
{[0.00]}\end{array}$ & $\begin{array}{c}0 \\
(0.00) \\
{[0.00]}\end{array}$ & $\begin{array}{c}0 \\
(0.00) \\
{[0.00]}\end{array}$ \\
\hline Hysteresis coil (W) & $\begin{array}{c}0 \\
(0.00) \\
{[0.00]}\end{array}$ & $\begin{array}{c}0 \\
(0: 00) \\
{[0.00]}\end{array}$ & $\begin{array}{c}0 \\
(0.00) \\
{[0.00]}\end{array}$ \\
\hline Eddy-current coil (W) & $\begin{array}{l}72.93 \\
(0.11) \\
{[0.11]}\end{array}$ & $\begin{array}{l}176.9 \\
(0.12) \\
{[0.12]}\end{array}$ & $\begin{array}{l}532.3 \\
(0.12) \\
{[0.12]}\end{array}$ \\
\hline Leakage loss (W) & $\begin{array}{l}559.6 \\
(0.87) \\
{[0.87]}\end{array}$ & $\begin{array}{l}1,385 \\
(0.93) \\
{[0.93]}\end{array}$ & $\begin{array}{l}5,663 \\
(1.23) \\
{[1.23]}\end{array}$ \\
\hline Dielectric loss (W) & $\begin{array}{l}1.758 \\
(0.00) \\
{[0.00]}\end{array}$ & $\begin{array}{l}3.258 \\
(0.00) \\
{[0.00]}\end{array}$ & $\begin{array}{l}17.29 \\
(0.00) \\
{[0.00]}\end{array}$ \\
\hline Total thermal loss (W) & 64,380 & 148,300 & 459,100 \\
\hline Total electrical loss (W) & 64,380 & 148,300 & 459,100 \\
\hline Cryocooler COP & NA & NA & NA \\
\hline
\end{tabular}

(a) Percentage of total thermal losses shown in parentheses ( ).

(b) Percentage of total electric losses shown in square brackets [ ]. 
Conventional step-up transformer full-load performance for the minimum life-cycle cost designs is shown in Table 3.6 and Table 3.7 for the conventional and amorphous cores, respectively.

As expected, the efficiencies of the step-up transformers are somewhat lower than those of comparably sized autotransformers. Efficiency is less in step-up transformers because all of the energy must be transferred through the transformer core and windings. With autotransformers, a portion of the energy is passed through the interconnected primary and secondary windings. Because all the current passes through the windings in a step-up transformer, the conductor loss represents a much larger fraction of the total losses: greater than $65 \%$ of the total losses for conventional core transformers and better than $90 \%$ of the total losses for amorphous core transformers.

As with the grid transformers, core and leakage loss make up the balance of the major losses in step-up transformers. Similarly, amorphous core designs have core losses that are less than onequarter of those occurring with conventional cores. This decrease in core loss results in efficiency improvement of better than $0.02 \%$ for all the step-up designs. This improved efficiency translates to a reduction in losses of about $10 \%$ to $20 \%$.

Design information for the conventional transformers is shown in Table 3.8. The optimal peak core flux density for all the amorphous core designs is 1.4 tesla. This is somewhat lower than the peak core flux density of the conventional core designs and results in larger core radii and higher core weights. The larger core radii and lower peak core flux densities, in turn, contribute to increased winding weights because of a longer mean-length-of turn, although this effect is mitigated somewhat by the fewer number of turns required. Thus, the higher efficiency of the amorphous core designs comes at the cost of increased material requirements.

\subsubsection{Base Case Cryostable Transformer Design and Performance Results}

The reason for considering superconducting transformer designs is to eliminate the load losses (conductor losses) associated with transformer operation. As the data in Section 3.6.1 demonstrated, load losses are the major loss in conventional transformers. Because of the zero resistivity of superconducting windings, superconducting transformer designs do not have load losses. However, in the superconducting designs, other losses that were insignificant in the conventional designs become more critical and represent a larger faction of the total losses. Other losses associated only with superconducting designs (hysteresis loss in the superconductor and heat leak into the cryostat) become important as well.

The full-load performance of the base case cryostable designs with conventional cores is shown in Tables 3.9 and 3.10. The efficiency of the minimum life-cycle cost cryostable designs with conventional cores is dramatically increased over that of comparable conventional transformer designs. Efficiency increases range from $0.04 \%$ to $0.15 \%$, which translates to a reduction in losses that ranges from $53 \%$ to $70 \%$. The efficiency increase results from the elimination of the conductor PR losses and a steep decline in the absolute magnitude of the core losses (a 65\% to 80\% decrease) for all the base case transformers. Core and leakage losses now make up at least three-quarters of the total thermal losses. 
Table 3.6. Conventional Step-Up Transformer Performance; Conventional Core

\begin{tabular}{lcc}
\multicolumn{3}{c}{ Transformer Rating } \\
\hline $63 \mathrm{MVA}$ & $315 \mathrm{MVA}$ & $630 \mathrm{MVA}$
\end{tabular}

Efficiency (\%)

99.756

99.803

99.880

Core loss (W)

35,890

$(23.31)^{())}$

$[23.31]^{(0)}$

122,600

(19.69)

[19.69]

210,400

(27.82)

[27.82]

Conductor loss (W)

110,400

463,700

(74.45)

(71.69)

[74.45]

512,500

[71.69]

(67.76)

[67.76]

Cryo heat leak (W)

0

$(0.00)$

0

0

[0.00]

$(0.00)$

[0.00]

$(0.00)$

[0.00]

Hysteresis coil (W)

0
$(0.00)$

[0.00]

0

$(0.00)$

[0.00]

$(0.00)$

[0.00]

Eddy-current coil (W)

131.8

$(0.09)$

632.6

692.6

[0.09]

(0.10)

[0.10]

$(0.09)$

[0.09]

Leakage loss (W)

\section{7,648}

(4.97)

35,800

(5.75)

[5.75]

32,740

[4.97]

(4.33)

[4.33]

Dielectric loss (W)

$\begin{array}{ll}3.580 & 15.41 \\ (0.00) & (0.00) \\ {[0.00]} & {[0.00]}\end{array}$

28.51

$(0.00)$

[0.00]

Total thermal loss (W)

154,000

622,800

756,300

Total electrical loss (W)

154,000

622,800

756,300

Cryocooler COP

NA

NA

NA

(a) Percentage of total thermal losses shown in parentheses ( ).

(b) Percentage of total electric losses shown in square brackets [ ]. 
Table 3.7. Conventional Step-Up Transformer Performance; Amorphous Core

\begin{tabular}{|c|c|c|c|}
\hline & & ansformer $\mathbf{R}$ & \\
\hline & $63 \mathrm{MVA}$ & 315 MVA & 630 MVA \\
\hline Efficiency (\%) & 99.777 & 99.826 & 99.906 \\
\hline Core loss (W) & $\begin{array}{c}6,452 \\
(4.57)^{(\omega)} \\
{[4.57]^{(0)}}\end{array}$ & $\begin{array}{l}22,440 \\
(4.08) \\
{[4.08]}\end{array}$ & $\begin{array}{l}47,720 \\
(8.06) \\
{[8.06]}\end{array}$ \\
\hline Conductor loss (W) & $\begin{array}{l}133,700 \\
(94.76) \\
{[94.76]}\end{array}$ & $\begin{array}{l}524,400 \\
(95.28) \\
{[95.28]}\end{array}$ & $\begin{array}{c}537,500 \\
(90.78) \\
{[90.78]}\end{array}$ \\
\hline Cryo heat leak (W) & $\begin{array}{c}0 \\
(0.00) \\
{[0.00]}\end{array}$ & $\begin{array}{c}0 \\
(0.00) \\
{[0.00]}\end{array}$ & $\begin{array}{c}0 \\
(0.00) \\
{[0.00]}\end{array}$ \\
\hline Hysteresis coil (W) & $\begin{array}{c}0 \\
(0.00) \\
{[0.00]}\end{array}$ & $\begin{array}{c}0 \\
(0.00) \\
{[0.00]}\end{array}$ & $\begin{array}{c}0 \\
(0.00) \\
{[0.00]}\end{array}$ \\
\hline Eddy-current coil (W) & $\begin{array}{l}151.8 \\
(0.11) \\
{[0.11]}\end{array}$ & $\begin{array}{l}681.8 \\
(0.12) \\
{[0.12]}\end{array}$ & $\begin{array}{l}699.7 \\
(0.12) \\
{[0.12]}\end{array}$ \\
\hline Leakage loss (W) & $\begin{array}{l}827.3 \\
(0.59) \\
{[0.59]}\end{array}$ & $\begin{array}{l}2,877 \\
(0.52) \\
{[0.52]}\end{array}$ & $\begin{array}{l}6,120 \\
(1.03) \\
{[1.03]}\end{array}$ \\
\hline Dielectric loss (W) & $\begin{array}{l}3.937 \\
(0.00) \\
{[0.00]}\end{array}$ & $\begin{array}{l}17.10 \\
(0.00) \\
{[0.00]}\end{array}$ & $\begin{array}{l}30.39 \\
(0.01) \\
{[0.01]}\end{array}$ \\
\hline Total thermal loss (W) & 141,100 & 550,400 & 592,100 \\
\hline Total electrical loss (W) & 141,100 & 550,400 & 592,100 \\
\hline Cryocooler COP & NA & NA & NA \\
\hline
\end{tabular}

(a) Percentage of total thermal losses shown in parentheses ().

(b) Percentage of total electric losses shown in square brackets [ ]. 
Table 3.8. Conventional Transformer Design Results

Core Technology and

Capacity Rating. MVA
Core Flux

Density. T
Core

Conventional - 1000

Amorphous - 30

Amorphous - 200

Amorphous - 1000

Step-Up Transformers

Conventional -63

Conventional -315

Conventional -630

Amorphous - 63
Amorphous - 315
Amorphous - 630
0.31

0.41

0.65

1.50

1.50

1.45

1.40

1.40

0.35

0.47

1.40

0.75

1.55

1.60

0.36

0.5

1.50

0.66

1.40

1.40

1.40
Core

Weight. kg
Copper

Weight. kg Weight. $\mathrm{kg}$
HTS

$\begin{array}{lrrrrr}\text { Conventional - 30 } & 1.50 & 0.31 & 16,580 & 5,068 & 0 \\ \text { Conventional - 200 } & 1.50 & 0.41 & 39,560 & 12,730 & 0 \\ \text { Conventional - 1000 } & 1.45 & 0.65 & 158,600 & 54,610 & 0 \\ \text { Amorphous - 30 } & 1.40 & 0.35 & 19,260 & 5,596 & 0 \\ \text { Amorphous - 200 } & 1.40 & 0.47 & 47,680 & 14,280 & 0 \\ \text { Amorphous - 1000 } & 1.40 & 0.75 & 194,900 & 55,910 & 0\end{array}$

This is not to say that the other losses are not significant; in fact, they are quite important. All the other losses must be removed by the cooling system, so the effect of these losses on the transformer efficiency is very significant. In addition to the electrical energy that generated the thermal loss, energy is consumed by the cryocooler to remove these losses for the cryostat. The thermal energy removed from the cryostat requires electrical energy of an amount equal to the thermal loss divided by the COP. Thus, for the designs in Tables 3.9 and 3.10, losses that must be removed by the cooling system are more costly from an efficiency standpoint than core or leakage losses from the refrigerator COP.

Eddy-current loss in the stabilizer (including cryocooler energy required to remove the heat from the cryostat) dominates the electrical losses for the base case designs shown in Tables 3.9 and 3.10, typically representing more than $50 \%$ of the total electrical losses. Hysteresis losses in the superconducting material are also significant, accounting for $9 \%$ to $18 \%$ of the total electrical losses. Heat leak into the cryostat accounts for $4 \%$ to $9 \%$ of the total electrical losses.

Tables 3.11 and 3.12 show the full load performance of the base case cryostable designs with amorphous cores. As with the conventional core cryostable designs, the efficiency of the minimum life-cycle cost cryostable designs with amorphous cores is dramatically increased over that of the conventional transformer designs with amorphous cores. Efficiency increases range from $0.04 \%$ to $0.13 \%$, which translates to a $56 \%$ to $69 \%$ reduction in losses. Similar to the conventional core 
Table 3.9. Cryostable Grid Transformer Performance; Conventional Core

(a) Percentage of total thermal losses shown in parentheses ().

(b) Percentage of total electric losses shown in square brackets [ ]. 
Table 3.10. Cryostable Step-Up Transformer Performance; Conventional Core

\begin{tabular}{|c|c|c|c|}
\hline & & nformer Ra & \\
\hline & 63 MVA & $315 \mathrm{MVA}$ & 630 MYA \\
\hline Efficiency (\%) & 99.887 & 99.940 & 99.951 \\
\hline Core loss (W) & $\begin{array}{c}12,680 \\
(45.12)^{(\omega)} \\
{[17.72]^{())}}\end{array}$ & $\begin{array}{l}38,230 \\
(61.70) \\
{[20.10]}\end{array}$ & $\begin{array}{l}41,340 \\
(51.57) \\
{[13.42]}\end{array}$ \\
\hline Conductor loss (W) & $\begin{array}{c}0 \\
(0.00) \\
{[0.00]}\end{array}$ & $\begin{array}{c}0 \\
(0.00) \\
{[0.00]}\end{array}$ & $\begin{array}{c}0 \\
(0.00) \\
{[0.00]}\end{array}$ \\
\hline Cryo heat leak (W) & $\begin{array}{l}399.9 \\
(1.42) \\
{[8.66]}\end{array}$ & $\begin{array}{l}1,226 \\
(1.98) \\
{[8.54]}\end{array}$ & $\begin{array}{l}2,313 \\
(2.89) \\
{[9.25]}\end{array}$ \\
\hline Hysteresis coil (W) & $\begin{array}{l}387.5 \\
(1.38) \\
{[8.93]}\end{array}$ & $\begin{array}{l}1,198 \\
(1.93) \\
{[8.98]}\end{array}$ & $\begin{array}{l}2,136 \\
(2.66) \\
{[9.24]}\end{array}$ \\
\hline Eddy current coil (W) & $\begin{array}{c}2,017 \\
(7.18) \\
{[46.48]}\end{array}$ & $\begin{array}{c}7,249 \\
(11.70) \\
{[54.32]}\end{array}$ & $\begin{array}{l}14,040 \\
(17.51) \\
{[60.71]}\end{array}$ \\
\hline Leakage loss (W) & $\begin{array}{l}13,020 \\
(46.33) \\
{[18.20]}\end{array}$ & $\begin{array}{c}15,280 \\
(24.66) \\
{[8.03]}\end{array}$ & $\begin{array}{c}22,640 \\
(28.24) \\
{[7.35]}\end{array}$ \\
\hline Dielectric loss (W) & $\begin{array}{l}0.4134 \\
(0.00) \\
{[0.01]}\end{array}$ & $\begin{array}{l}2.797 \\
(0.00) \\
{[0.02]}\end{array}$ & $\begin{array}{l}4.820 \\
(0.01) \\
{[0.02]}\end{array}$ \\
\hline Total thermal loss (W) & 28,100 & 61,960 & 80,160 \\
\hline Total electrical loss (W) & 71,540 & 190,200 & 308,100 \\
\hline Cryocooler COP & 0.06458 & 0.07546 & 0.08115 \\
\hline
\end{tabular}

(a) Percentage of total thermal losses shown in parentheses ( ).

(b) Percentage of total electric losses shown in square brackets []. 
Table 3.11. Cryostable Grid Transformer Performance; Amorphous Core

\begin{tabular}{ccc}
\multicolumn{3}{c}{ Transformer Rating } \\
\hline $30 \mathrm{MVA}$ & $200 \mathrm{MVA}$ & $1000 \mathrm{MVA}$
\end{tabular}

\begin{tabular}{|c|c|c|c|}
\hline Efficiency (\%) & 99.905 & 99.970 & 99.982 \\
\hline Core loss (W) & $\begin{array}{c}1,264 \\
(44.70)^{(4)} \\
{[4.41]^{(0)}}\end{array}$ & $\begin{array}{c}3,032 \\
(44.35) \\
{[5.05]}\end{array}$ & $\begin{array}{c}11,620 \\
(47.49) \\
{[6.62]}\end{array}$ \\
\hline Conductor loss (W) & $\begin{array}{c}0 \\
(0.00) \\
{[0.00]}\end{array}$ & $\begin{array}{c}0 \\
(0.00) \\
{[0.00]}\end{array}$ & $\begin{array}{c}0 \\
(0.00) \\
{[0.00]}\end{array}$ \\
\hline Cryo heat leak (W) & $\begin{array}{l}126.8 \\
(4.48) \\
{[7.48]}\end{array}$ & $\begin{array}{l}313.8 \\
(4.59) \\
{[7.46]}\end{array}$ & $\begin{array}{l}787.9 \\
(3.22) \\
{[5.58]}\end{array}$ \\
\hline Hysteresis coil (W) & $\begin{array}{c}366.2 \\
(12.95) \\
{[22.87]}\end{array}$ & $\begin{array}{c}764.6 \\
(11.18) \\
{[19.44]}\end{array}$ & $\begin{array}{c}2,253 \\
(9.21) \\
{[17.25]}\end{array}$ \\
\hline Eddy current coil (W) & $\begin{array}{c}1,035 \\
(36.60) \\
{[64.64]}\end{array}$ & $\begin{array}{c}2,651 \\
(38.78) \\
{[67.40]}\end{array}$ & $\begin{array}{c}9,101 \\
(37.19) \\
{[69.68]}\end{array}$ \\
\hline Leakage loss (W) & $\begin{array}{l}162.1 \\
(5.73) \\
{[0.57]}\end{array}$ & $\begin{array}{l}388.8 \\
(5.69) \\
{[0.65]}\end{array}$ & $\begin{array}{l}1,490 \\
(6.09) \\
{[0.85]}\end{array}$ \\
\hline Dielectric loss (W) & $\begin{array}{l}0.1807 \\
(0.01) \\
{[0.01]}\end{array}$ & $\begin{array}{l}0.2609 \\
(0.00) \\
{[0.01]}\end{array}$ & $\begin{array}{l}1.769 \\
(0.01) \\
{[0.01]}\end{array}$ \\
\hline Total thermal loss (W) & 2,828 & 6,836 & 24,470 \\
\hline Total electrical loss (W) & 28,630 & 60,090 & 175,500 \\
\hline Cryocooler COP & 0.05924 & 0.07004 & 0.08041 \\
\hline
\end{tabular}

(a) Percentage of total thermal losses shown in parentheses ( ).

(b) Percentage of total electric losses shown in square brackets [ ]. 
Table 3.12. Cryostable Step-Up Transformer Performance; Amorphous Core

\begin{tabular}{|c|c|c|c|}
\hline & \multicolumn{3}{|c|}{ Transformer Rating } \\
\hline & $63 \mathrm{MVA}$ & $315 \mathrm{MVA}$ & 630MVA \\
\hline Efficiency (percent) & 99.909 & 99.945 & 99.963 \\
\hline Core loss (W) & $\begin{array}{c}1,603 \\
(32.68)^{(a)} \\
{[2.78]^{(0)}}\end{array}$ & $\begin{array}{c}5,425 \\
(31.95) \\
{[3.13]}\end{array}$ & $\begin{array}{c}11,810 \\
(42.87) \\
{[5.03]}\end{array}$ \\
\hline Conductor loss (W) & $\begin{array}{c}0 \\
(0.00) \\
{[0.00]}\end{array}$ & $\begin{array}{c}0 \\
(0.00) \\
{[0.00]}\end{array}$ & $\begin{array}{c}0 \\
(0.00) \\
{[0.00]}\end{array}$ \\
\hline Cryo heat leak (W) & $\begin{array}{c}410.1 \\
(8.36) \\
{[10.70]}\end{array}$ & $\begin{array}{l}1244 \\
(7.33) \\
{[9.27]}\end{array}$ & $\begin{array}{c}2380 \\
(8.64) \\
{[12.64]}\end{array}$ \\
\hline Hysteresis coil (W) & $\begin{array}{c}505.4 \\
(10.30) \\
{[14.06]}\end{array}$ & $\begin{array}{c}1549 \\
(9.12) \\
{[12.44]}\end{array}$ & $\begin{array}{c}1894 \\
(6.87) \\
{[10.87]}\end{array}$ \\
\hline Eddy current coil (W) & $\begin{array}{c}2,590 \\
\mathbf{( 5 2 . 8 0 )} \\
{[72.07]}\end{array}$ & $\begin{array}{c}9,308 \\
(54.82) \\
{[74.75]}\end{array}$ & $\begin{array}{l}12,330 \\
(44.75) \\
{[70.75]}\end{array}$ \\
\hline Leakage loss (W) & $\begin{array}{l}205.6 \\
(4.19) \\
{[0.36]}\end{array}$ & $\begin{array}{l}695.6 \\
(4.10) \\
{[0.40]}\end{array}$ & $\begin{array}{l}1,514 \\
(5.50) \\
{[0.65]}\end{array}$ \\
\hline Dielectric loss (W) & $\begin{array}{c}0.4910 \\
(0.01) \\
{[0.01]}\end{array}$ & $\begin{array}{l}3.286 \\
(0.02) \\
{[0.03]}\end{array}$ & $\begin{array}{l}4.485 \\
(0.02) \\
{[0.03]}\end{array}$ \\
\hline Total thermal loss (W) & 4,905 & 16,980 & 27,550 \\
\hline Total electrical loss (W) & 57,600 & 173,300 & 234,700 \\
\hline Cryocooler COP & 0.06654 & 0.07742 & 0.08021 \\
\hline
\end{tabular}

(a) Percentage of total thermal losses shown in parentheses ( ).

(b) Percentage of total electric losses shown in square brackets [ ]. 
designs, the increase in efficiency of the amorphous core designs is due to the elimination of the conductor $\mathrm{I}^{2} \mathrm{R}$ losses and a steep decline in the absolute magnitude of the core losses (a $72 \%$ to $76 \%$ decrease) for all transformer sizes. Core and leakage losses now constitute $36 \%$ to $54 \%$ of the total thermal losses, significantly less than the minimum $75 \%$ that these losses represent in conventional core cryostable designs.

The decrease in the core loss is caused by a dramatic decrease in the total core weight that was partially offset by a small increase in the peak core flux density. (Compare Tables 3.8 and 3.13.) Because the operating current density of the superconductor is much higher than that of the copper in conventional designs, the core window areas and winding volumes for the cryostable designs are much smaller. Additionally, this higher current density results in designs that typically have more than twice the number of turns of the comparable conventional design. The higher number of turns allows the core radii to be reduced relative to the conventional designs with only a slight increase in core flux density. Thus, regardless of core type, the cryostable designs will have much smaller cores and associated core losses than conventional transformer designs.

The load on the cryocooler from the amorphous core designs is nearly the same as from the conventional core designs (the efficiency improvement is primarily due to reduced losses outside the cryostat). Thus, the cryocooler COPs for the amorphous core and convention core designs are nearly identical. Therefore, for all the base case cryostable designs (Tables 3.9 through 3.12), the losses removed by the thermal management system are 12 to 17 times more costly from an efficienry standpoint than losses outside the cryostat.

The design information for the base case cryostable transformer designs is shown in Table 3.13. By comparing the information in Table 3.13 with the conventional transformer design results in Table 3.8, we can make many interesting observations. As with the conventional transformer designs, the optimal peak core flux density for all the amorphous core designs is 1.4 tesla. Conversely, for the conventional core cryostable designs, the peak core flux density is much higher than in the amorphous core cryostable designs or the comparable conventional designs with conventional cores.

The core radius and core volume of the cryostable designs are much smaller than the comparable conventional designs, regardless of core type. The optimal designs for conventional core cryostable transformers have small core radii and high peak core flux densities because this yields smaller winding volumes and hence, lower losses inside the cryostat. The smaller core volumes more than compensate for the increased loss per unit volume (due to the higher peak core flux densities) and hence, core losses are less in the cryostable designs than in the comparable conventional designs. Because leakage flux increases with higher peak core flux densities, the decreased losses from the smaller winding volumes is somewhat offset by increased winding loss per unit volume. (Eddy currents in the stabilizer and hysteresis losses in the superconductor increase because they are induced, in part, by the leakage flux.)

Additionally, the stabilizer requirements (64\% to $88 \%$ of the winding weights for the base case designs) increase to roughly the $4 / 3$ power of the current. Thus, transformers with higher ratings (and currents) will require a higher percentage of stabilizer and, therefore, eddy currents will typically represent a larger fraction of the total losses. 
Table 3.13. Cryostable Transformer Design Results

\begin{tabular}{|c|c|c|c|c|c|}
\hline $\begin{array}{l}\text { Core Technology and } \\
\text { Capacity Rating. MVA }\end{array}$ & $\begin{array}{l}\text { Core Flux } \\
\text { Density. T }\end{array}$ & $\begin{array}{c}\text { Core } \\
\text { Radius.m }\end{array}$ & $\begin{array}{c}\text { Core } \\
\text { Weight. kg }\end{array}$ & $\begin{array}{c}\text { Copper } \\
\text { Weight. kg }\end{array}$ & $\begin{array}{c}\text { HTS } \\
\text { Weight. kg }\end{array}$ \\
\hline \multicolumn{6}{|l|}{ Grid Transformers } \\
\hline Conventional - 30 & 1.60 & 0.22 & 4,091 & 30.37 & 16.85 \\
\hline Conventional - 200 & 1.55 & 0.30 & 10,250 & 104.20 & 41.13 \\
\hline Conventional - 1000 & 1.60 & 0.38 & 24,360 & 655.60 & 181.10 \\
\hline Amorphous - 30 & 1.40 & 0.26 & 5,578 & 33.62 & 18.67 \\
\hline Amorphous - 200 & 1.40 & 0.35 & 13,380 & 114.30 & 45.09 \\
\hline Amorphous - 1000 & 1.40 & 0.54 & 51,300 & 589.00 & 162.80 \\
\hline \multicolumn{6}{|l|}{ Step-Up Transformers } \\
\hline Conventional - 63 & 1.80 & 0.24 & 5,959 & 80.28 & 20.48 \\
\hline Conventional - 315 & 1.65 & 0.37 & 23,850 & 426.10 & 74.64 \\
\hline Conventional - 630 & 1.70 & 0.35 & 23,470 & $1,150.00$ & 157.40 \\
\hline Amorphous - 63 & 1.40 & 0.27 & 7,077 & 106.70 & 27.16 \\
\hline Amorphous - 315 & 1.40 & 0.39 & 23,940 & 556.30 & 97.30 \\
\hline Amorphous - 630 & 1.40 & 0.52 & 52,110 & $1,057.00$ & 142.50 \\
\hline
\end{tabular}

\subsubsection{Base Case Ultrastable Transformer Design and Performance Results}

As with the cryostable designs, the efficiencies of the ultrastable designs are substantially higher than those of the conventional designs. Efficiencies for the ultrastable designs are significantly higher than for the cryostable designs; however, material requirements are also substantially higher.

The full-load performance of the base case ultrastable designs with conventional cores is shown in Tables 3.14 and 3.15. The efficiency of the minimum life-cycle cost ultrastable designs with conventional cores is dramatically increased over that of the conventional and cryostable designs. Efficiency increases over the conventional designs range from $0.05 \%$ to $0.19 \%$ which translates to a $65 \%$ to $77 \%$ reduction in losses. The comparison of the base case ultrastable and cryostable designs with conventional cores is less dramatic; efficiency increases range from $0.01 \%$ to $0.03 \%$, which is equivalent to a substantial loss reduction of $24 \%$ to $34 \%$.

The efficiency increase over conventional designs with conventional cores occurs for the same reason as with the cryostable designs: conductor $I^{2} \mathrm{R}$ losses are eliminated and the absolute magnitude of the core losses decline sharply (a 56\% to 68\% decrease) for all base case designs. Core and leakage losses now make up at least three-quarters of the total thermal losses (38\% to $52 \%$ of the total electrical losses). As with the cryostable designs, the core and leakage losses are preferable to other 
Table 3.14. Ultrastable Grid Transformer Performance; Conventional Core

\begin{tabular}{lcc}
\multicolumn{3}{c}{ Transformer Rating } \\
\hline $30 \mathrm{MVA} \quad 200 \mathrm{MVA}$ & $1000 \mathrm{MVA}$
\end{tabular}

\begin{tabular}{|c|c|c|c|}
\hline Efficiency (\%) & 99.923 & 99.974 & 99.984 \\
\hline Core loss (W) & $\begin{array}{c}7,258 \\
(76.93)^{(0)} \\
{[31.38]^{(0)}}\end{array}$ & $\begin{array}{l}17,340 \\
(76.93) \\
{[33.70]}\end{array}$ & $\begin{array}{l}60,490 \\
(80.16) \\
{[37.57]}\end{array}$ \\
\hline Conductor loss (W) & $\begin{array}{c}0 \\
(0.00) \\
{[0.00]}\end{array}$ & $\begin{array}{c}0 \\
(0.00) \\
{[0.00]}\end{array}$ & $\begin{array}{c}0 \\
(0.00) \\
{[0.00]}\end{array}$ \\
\hline Cryo heat leak. (W) & $\begin{array}{c}140.2 \\
(1.49) \\
{[10.79]}\end{array}$ & $\begin{array}{c}336.3 \\
(1.49) \\
{[10.24]}\end{array}$ & $\begin{array}{l}836.0 \\
(1.11) \\
{[6.94]}\end{array}$ \\
\hline Hysteresis coil (W) & $\begin{array}{c}628.6 \\
(6.66) \\
{[51.11]}\end{array}$ & $\begin{array}{c}1,509 \\
(6.69) \\
{[46.86]}\end{array}$ & $\begin{array}{c}5,557 \\
(7.36) \\
{[49.61]}\end{array}$ \\
\hline Eddy-current coil (W) & $\begin{array}{c}0 \\
(0.00) \\
{[0.00]}\end{array}$ & $\begin{array}{c}0 \\
(0.00) \\
{[0.00]}\end{array}$ & $\begin{array}{c}0 \\
(0.00) \\
{[0.00]}\end{array}$ \\
\hline Leakage loss (W) & $\begin{array}{c}1,547 \\
(16.40) \\
{[6.69]}\end{array}$ & $\begin{array}{c}3,694 \\
(16.39) \\
{[7.18]}\end{array}$ & $\begin{array}{c}9,411 \\
(12.47) \\
{[5.85]}\end{array}$ \\
\hline Dielectric loss (W) & $\begin{array}{l}0.2900 \\
(0.00) \\
{[0.02]}\end{array}$ & $\begin{array}{c}0.3572 \\
(0.00) \\
{[0.01]}\end{array}$ & $\begin{array}{l}2.451 \\
(0.00) \\
{[0.02]}\end{array}$ \\
\hline Total thermal loss (W) & 9,434 & 22,540 & 75,460 \\
\hline Total electrical loss (W) & 23,130 & 51,450 & 161,000 \\
\hline Cryocooler COP & 0.05616 & 0.06386 & 0.07477 \\
\hline
\end{tabular}

(a) Percentage of total thermal losses shown in parentheses ( ).

(b) Percentage of total electric losses shown in square brackets [ ]. 
Table 3.15. Ultrastable Step-Up Transformer Performance; Conventional Core

\begin{tabular}{|c|c|c|c|}
\hline & \multicolumn{3}{|c|}{ Transformer Rating } \\
\hline & $63 \mathrm{MVA}$ & $315 \mathrm{MVA}$ & $630 \mathrm{MVA}$ \\
\hline Efficiency (\%) & 99.915 & 99.954 & 99.968 \\
\hline Core loss (W) & $\begin{array}{c}15,930 \\
(55.10)^{(1)} \\
{[29.68]^{())}}\end{array}$ & $\begin{array}{l}41,340 \\
(60.74) \\
{[28.71]}\end{array}$ & $\begin{array}{l}66,800 \\
(76.45) \\
{[32.63]}\end{array}$ \\
\hline Conductor loss (W) & $\begin{array}{c}0 \\
(0.00) \\
{[0.00]}\end{array}$ & $\begin{array}{c}0 \\
(0.00) \\
{[0.00]}\end{array}$ & $\begin{array}{c}0 \\
(0.00) \\
{[0.00]}\end{array}$ \\
\hline Cryo heat leak (W) & $\begin{array}{c}423.2 \\
(1.46) \\
{[13.40]}\end{array}$ & $\begin{array}{c}1,260 \\
(1.85) \\
{[12.46]}\end{array}$ & $\begin{array}{c}2,396 \\
(2.74) \\
{[15.70]}\end{array}$ \\
\hline Hysteresis coil (W) & $\begin{array}{c}1,033 \\
(3.57) \\
{[64.64]}\end{array}$ & $\begin{array}{c}4,072 \\
(5.98) \\
{[43.09]}\end{array}$ & $\begin{array}{c}6,343 \\
(7.26) \\
{[44.65]}\end{array}$ \\
\hline Eddy current coil (W) & $\begin{array}{c}0 \\
(0.00) \\
{[0.00]}\end{array}$ & $\begin{array}{c}0 \\
(0.00) \\
{[0.00]}\end{array}$ & $\begin{array}{c}0 \\
(0.00) \\
{[0.00]}\end{array}$ \\
\hline Leakage loss (W) & $\begin{array}{l}11,950 \\
(41.34) \\
{[22.26]}\end{array}$ & $\begin{array}{l}22,640 \\
(33.26) \\
{[15.72]}\end{array}$ & $\begin{array}{c}14,230 \\
(16.29) \\
{[6.95]}\end{array}$ \\
\hline Dielectric loss (W) & $\begin{array}{l}0.6456 \\
(0.00) \\
{[0.02]}\end{array}$ & $\begin{array}{l}4.158 \\
(0.01) \\
{[0.04]}\end{array}$ & $\begin{array}{l}5.057 \\
(0.01) \\
{[0.04]}\end{array}$ \\
\hline Total thermal loss (W) & 28,910 & 68,060 & 87,380 \\
\hline Total electrical loss (W) & 53,680 & 144,000 & 204,700 \\
\hline Cryocooler COP & 0.05882 & 0.07023 & 0.07457 \\
\hline
\end{tabular}

(a) Percentage of total thermal losses shown in parentheses ( ).

(b) Percentage of total electric losses shown in square brackets [ ]. 
losses because all other losses are removed by the cooling system. The balance of the losses is due predominantly to the heat leak into the cryostat and to hysteresis losses in the superconductor.

Hysteresis losses in the superconductor (including cryocooler energy required to remove heat from the cryostat) dominate the electrical losses for the base case designs shown in Tables 3.14 and 3.15. These losses represent between $35 \%$ and $51 \%$ of the total electrical losses. The percentage of the total electrical losses attributed to heat leak into the cryostat is $7 \%$ to $16 \%$.

The efficiencies for the ultrastable designs are higher than those for the cryostable designs because of one factor-elimination of the eddy-current loss in the stabilizer. All other losses removed by the cryostat are higher for the base case ultrastable designs with conventional cores than the comparable cryostable designs. However, these increases are minor compared with the reduced losses achieved by eliminating the stabilizer metal.

Tables 3.16 and 3.17 show the full-load performance of the base case ultrastable designs with amorphous cores. As with the conventional core ultrastable designs, the efficiency of the minimum life-cycle cost ultrastable designs with amorphous cores is dramatically increased over that of the conventional designs with amorphous cores. Efficiency increases range from $0.04 \%$ to $0.17 \%$, which translates to a $74 \%$ to $81 \%$ reduction in losses. The comparison between the base case ultrastable and cryostable designs with amorphous cores is less dramatic; efficiency increases ranged from $0.01 \%$ to $0.04 \%$, which is equivalent to a substantial loss reduction of $37 \%$ to $43 \%$.

As before, the increase in efficiency of the amorphous core designs is a result of elimination of the conductor $\mathrm{I}^{2} \mathrm{R}$ losses and a steep decline in the absolute magnitude of the core losses (a $63 \%$ to $76 \%$ decrease) for all transformer sizes. Core and leakage losses now constitute $53 \%$ to $73 \%$ of the total thermal losses, considerably less than the minimum 75\% that these losses represent in conventional core ultrastable designs.

The decrease in core loss is caused by a dramatic decrease in the total core weight with only a small increase in the peak core flux density (see Tables 3.8 and 3.18). As with the cryostable designs, the operating current density of the superconductor is much higher than that of copper in conventional designs. Thus, the core window areas and winding volumes for the ultrastable designs are much smaller. This higher current density results in designs that typically have more than twice the number of turns in comparable conventional design. The higher number of turns allows the core radii to be reduced relative to the conventional designs with only a slight increase in core flux density. Thus, regardless of core type, the ultrastable designs will have much smaller cores and associated core losses.

Again, the remaining losses are caused predominantly by to the heat leak into the cryostat and hysteresis losses in the material. Hysteresis loss in the material dominates the electrical losses (including cryocooler energy required to remove heat from the cryostat) for the base case designs shown in Tables 3.16 and 3.17. These losses represent between $67 \%$ and $77 \%$ of the total electrical losses. The total electrical losses attributed to heat leak into the cryostat for these designs are a substantial $10 \%$ to $22 \%$. 
Table 3.16. Ultrastable Grid Transformer Performance; Amorphous Core

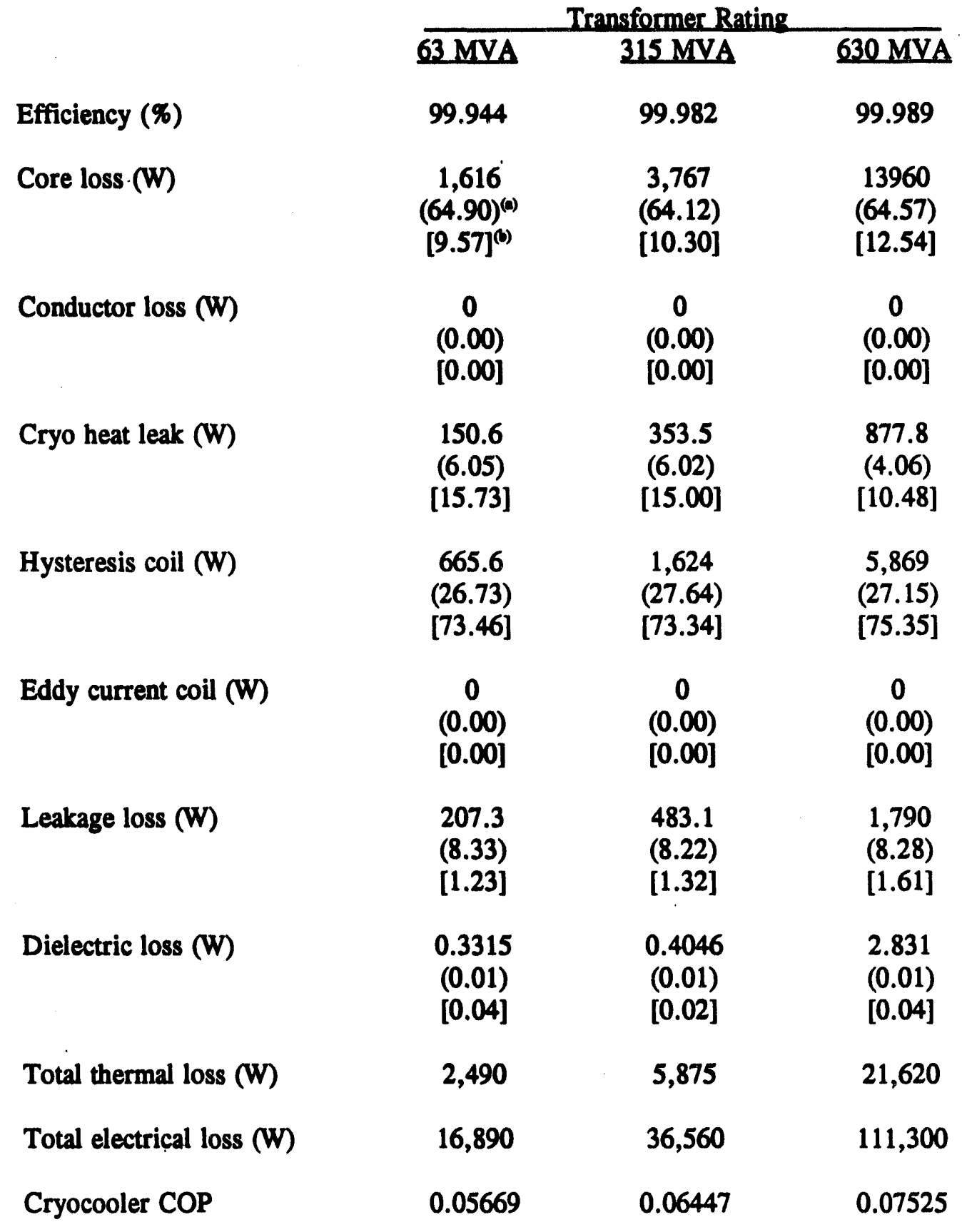

(a) Percentage of total thermal losses shown in parentheses ( ).

(b) Percentage of total electric losses shown in square brackets [ ]. 
Table 3.17. Ultrastable Step-Up Transformer Performance; Amorphous Core

\begin{tabular}{|c|c|c|c|}
\hline & & isformer Rat & \\
\hline & $63 \mathrm{MVA}$ & 315MVA & 630MVA \\
\hline Efficiency (\%) & 99.948 & 99.967 & 99.977 \\
\hline Core loss (W) & $\begin{array}{c}2,014 \\
(55.77)^{(a)} \\
{[6.12]^{(b)}}\end{array}$ & $\begin{array}{c}5,301 \\
(46.70) \\
{[5.12]}\end{array}$ & $\begin{array}{c}13,920 \\
(61.40) \\
{[9.42]}\end{array}$ \\
\hline Conductor loss (W) & $\begin{array}{c}0 \\
(0.00) \\
{[0.00]}\end{array}$ & $\begin{array}{c}0 \\
(0.00) \\
{[0.00]}\end{array}$ & $\begin{array}{c}0 \\
(0.00) \\
{[0.00]}\end{array}$ \\
\hline Cryo heat leak (W) & $\begin{array}{c}435.3 \\
(12.05) \\
{[21.85]}\end{array}$ & $\begin{array}{c}1,281 \\
(11.29) \\
{[17.15]}\end{array}$ & $\begin{array}{c}2,439 \\
(10.76) \\
{[21.96]}\end{array}$ \\
\hline Hysteresis coil (W) & $\begin{array}{c}1,337 \\
(37.03) \\
{[71.18]}\end{array}$ & $\begin{array}{c}5,363 \\
(47.25) \\
{[76.99]}\end{array}$ & $\begin{array}{c}6,961 \\
(30.71) \\
{[67.38]}\end{array}$ \\
\hline Eddy current coil (W) & $\begin{array}{c}0 \\
(0.00) \\
{[0.00]}\end{array}$ & $\begin{array}{c}0 \\
(0.00) \\
{[0.00]}\end{array}$ & $\begin{array}{c}0 \\
(0.00) \\
{[0.00]}\end{array}$ \\
\hline Leakage loss (W) & $\begin{array}{l}258.3 \\
(7.15) \\
{[0.79]}\end{array}$ & $\begin{array}{l}679.8 \\
(5.99) \\
{[0.66]}\end{array}$ & $\begin{array}{l}1,785 \\
{[7.87]} \\
{[1.21]}\end{array}$ \\
\hline Dielectric loss (W) & $\begin{array}{l}0.7433 \\
(0.02) \\
{[0.04]}\end{array}$ & $\begin{array}{l}4.483 \\
(0.04) \\
{[0.06]}\end{array}$ & $\begin{array}{l}5.358 \\
(0.02) \\
{[0.05]}\end{array}$ \\
\hline Total thermal loss (W) & 3,611 & 11,350 & 22,670 \\
\hline Total electrical loss (W) & 32,900 & 103,500 & 147,700 \\
\hline Cryocooler COP & 0.06055 & 0.07216 & 0.07521 \\
\hline
\end{tabular}

(a) Percentage of total thermal losses shown in parentheses ( ).

(b) Percentage of total electric losses shown in square brackets [ ]. 
The load on the cooling system from the amorphous core designs is nearly the same as from the conventional core designs (the efficiency improvement stems primarily from reduced losses outside the cryostat). Thus, the cryocooler COPs of the amorphous core and the conventional core designs are nearly identical. Therefore, for all the base case ultrastable designs (Tables 3.14 through 3.17), the losses removed by the cooling system are 13 to 18 times more costly from an efficiency standpoint than losses outside the cryostat. Because the base case ultrastable designs are somewhat more efficient than the cryostable designs, the load on the cryocooler is smaller and, hence, the COPs are lower. That is, the cost penalty for removing heat from the cryostat is higher for the ultrastable designs than for the comparable cryostable designs.

Table 3.18 contains the design information for the base case ultrastable transformer designs. Comparing the information in Table 3.18 with the conventional transformer design results in Table 3.8 and the cryostable transformer design information in Table 3.13 results in the following observations. As with the conventional and cryostable designs, the optimal peak core flux density for all the amorphous core designs is 1.4 tesla. The peak core flux density for the ultrastable designs with conventional cores is higher than that of either the ultrastable designs with amorphous cores or the comparable conventional designs with conventional cores. The peak core flux densities in ultrastable designs with conventional cores is typically lower than that of cryostable designs with conventional cores.

Table 3.18. Ultrastable Transformer Design Results

Core Technology and

Capacity Rating, MVA
Core Flux Density, T
Core Radius, m
Core

Weight. kg
Copper

Weight. kg
HTS

Weight, kg

Grid Transformers

Conventional - 30

1.55

0.23

5,468

172.6

Conventional - 200

1.55

0.31

13,060

414.6

Conventional - 1000

1.50

0.48

50,080

$1,553.0$

Amorphous - 30

1.40

0.27

7,135

185.7

Amorphous - 200

1.40

0.36

16,630

453.2

Amorphous - 1000

1.40

0.556

16,300

$1,637.0$

Step-Up Transformers

Conventional - 63

Conventional - 315

1.70

0.35

23,470

787.7

Conventional - 630

1.55

0.47

50,320

$1,250.0$

Amorphous - 63

1.40

0.28

8,890

266.6

Amorphous - 315

1.40

0.37

23,400

0.54

61,430

$1,069.0$

1.40

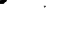


The core radius and core volume of the ultrastable designs are much smaller than the comparable conventional designs, regardless of core type; however, they are slightly larger than the comparable cryostable designs. As with the cryostable designs, the optimal designs for conventional core ultrastable transformers have smaller core radii, higher peak core flux densities, and smaller winding volumes than do conventional designs. Hence, losses inside the cryostat are smaller than they would be if cores sizes were similar to those used in conventional transformers. The smaller core volumes more than compensate for the increased losses per unit volume (because of the higher peak core flux densities), so core losses are lower in the ultrastable designs than in the comparable conventional designs. Because the leakage flux increases with core flux density, the decreased loss from the smaller winding volume is mitigated somewhat by increased winding loss per unit volume. (Hysteresis losses in the superconductor increase because they are induced, in part, by the leakage flux).

\subsection{Summary and Conclusions}

This chapter provides an insight into some important transformer design issues and discusses how these issues apply to HTS transformer design. The ideal transformer relationships were given, and the background and fundamentals of transformer operation were discussed. The approach used in the transformer design model was described, highlighting the technical design tradeoffs that can be made to minimize the life-cycle cost of both conventional and superconducting transformers. Major issues relating to superconducting transformer design were included, and equations used in the transformer design and performance analysis model were presented and discussed.

Transformer losses were categorized as being either load losses or no-load losses. The magnitudes of the load losses are dependent on the amount of load current flowing through the transformer, whereas the no-load loss components are independent of load current but may vary with terminal voltage, temperature, or other factors. Some of the loss mechanisms in conventional and superconducting transformers are identical (e.g., core loss), while other loss terms relate to one technology but not the other (e.g., I ${ }^{2} \mathrm{R}$ losses in conventional windings and hysteresis losses in superconducting windings).

The load loss term, caused by resistance to current flow in the windings of the transformer, typically comprises over $50 \%$ of the total transformer losses in the optimized conventional designs. Superconducting transformer designs are dominated by the no-load loss terms.

The only loss components in the superconducting transformer that might be classified as load loss terms are the hysteresis and eddy-current losses in the composite superconductor windings. These losses are dependent on the magnitude of the ac magnetic field to which the winding conductors are exposed. The self-induced magnetic field in the windings is dependent on the amount of current they carry and location in the transformer.

It was necessary to determine the magnetic self-field intensity at the conductors and its effect on the hysteresis and eddy-current losses in the superconducting transformer. A simplifying equation was developed that relates the average self-field intensity at the conductors to the current in the conductor and the ratio of the conductor strand radius to the total conductor radius. Using this 
equation, the self-induced magnetic field in the transformer windings becomes a major driving factor in the cost and performance of the superconducting transformer designs studied.

Losses for the superconducting grid transformers (autotransformers and step-down) are somewhat overstated. This is because hysteresis and eddy-current losses in the windings were modeled as constant (at full-load current), whereas, in actuality, these transformers spend much of the time operating at much less than full-load current.

The transformer design methodology develops conventional designs similar in configuration and performance to modern standard step-up, autotransformer, or step-down designs available from major manufacturers of power equipment. Superconducting transformers have been designed using the best available information on potential materials and performance with HTS superconductors. Also, reasonable engineering assumptions were made in an effort to design reliable, efficient, and cost-effective superconducting transformers.

Transformer losses, which are relatively unimportant in conventional transformers, become very important in superconducting transformer designs where the heat generated must be removed by the refrigeration system. Therefore, the design of superconductive transformers is very sensitive to the various loss mechanisms in the windings. Design tradeoffs that would not be prudent in conventional designs are cuscial to the viability of superconductive transformers.

To study the effect of the amount of stabilizer in the windings on the cost and performance of superconducting transformer designs, two design philosophies were evaluated. The two design strategies studied contained globally cryostabilized windings and highly redundant superconductive windings that omitted the stabilizer metal of cryostabilized designs.

Cryostable superconductive transformer designs evaluated in this study use shunt stabilizer to ensure recovery from a global quench of superconductivity. It was found that reliable cryostable superconductive transformer designs have the potential for smaller size, lower losses, higher efficiency, and lower life-cycle costs than conventional transformers.

A major power loss component in the cryostable transformer is due to eddy currents induced in the stabilizer. Superconducting transformer designs employing redundant superconductor materials rather than copper to ensure stable, reliable operation were also evaluated. The intent of this concept is to reduce total losses and possibly the materials cost of the superconducting transformer windings as well. These transformers have the additional benefit of not routinely switching to normal conductivity during brief surges and overloads, as does the cryostable transformer. For the purposes of this report, these transformers were designated "ultrastable" transformers.

The ultrastable transformer relies on the windings themselves to maintain thermal stability. Local disturbances in superconductivity are mitigated by the cooling fluid heat transfer. The possibility of a global quench is reduced by designing the conductors to operate at a small fraction of the critical current density. 
Ultrastable transformer designs have no eddy-current loss but do suffer increased hysteresis loss in the superconductor because of the increased volume of superconductor in the transformer windings. The base case ultrastable transformer designs studied were more efficient than comparable conventional and cryostable transformer designs at critical current densities of $10^{5} \mathrm{~A} / \mathrm{cm}^{2}$.

Both superconducting designs have the advantage of significantly reduced overall size and weight compared with conventional designs. As a result, superconducting transformers might first be used in military, aerospace, or other applications where size and weight constraints are important.

In most applications, it is likely that the stability requirements in a superconducting power transformer will best be met by a compromise between the ultrastable and cryostable designs. Any design will have to ensure that overall power system reliability is in no way sacrificed by the choice or implementation of the technology.

The reliability of power system components, including transformers, is at least as important to the electric utilities as performance and cost. The superconducting transformer system reliability could approach that of modern conventional transformers through the use of a nonmechanical cooling system. Such a system could employ a local cryogenic coolant reservoir that could be refilled periodically by a tanket truck. This is discussed in more detail in Chapter 4.

Key conclusions drawn from the results presented in this chapter follow.

- Amorphous cores dramatically decrease the core losses in conventional transformers. This occurs because the increased resistivity of the amorphous core material reduces the eddy currents in the core and the amorphous structure greatly reduces the hysteresis losses.

- For the same MVA rating in a conventional transformer, the optimal amorphous core design will have a larger core than the comparable conventional core design. Amorphous cores typically have lower flux densities and a lower stacking factor than laminated silicon steel cores. Thus, larger cores are to be expected.

- In superconducting designs with conventional cores, the peak core flux density will typically be greater than in the comparable conventional designs with conventional cores. A higher peak core flux density, combined with higher possible operating current densities in superconducting. winding, results in smaller core radii and much reduced winding volumes. Some of the losses inside the cryostat (eddy currents in the stabilizer and hysteresis losses in the superconductor) are induced, in part, by the leakage flux. The leakage flux then increases with core flux density. However, the decreased volume more than compensates for the increase in the per unit of mass losses.

- For the base-case assumptions, cryostable designs have much lower material requirements than comparable conventional designs and slightly lower material requirements than ultrastable designs. The operating current density of the ultrastable and cryostable (including superconducting and stability material) designs is much higher than that of the conventional designs. Because the operating current density of the superconductor is much higher than that of the copper in conventional designs, the core window areas and winding volumes for the superconducting designs are much smaller. This higher current density results in designs that 
typically have more than twice the number of turns of the comparable conventional design. The higher number of turns allows the core radii to be reduced relative to the conventional designs with only a slight increase in core flux density. Thus, regardless of core type, the superconducting designs will have much smaller cores and associated core losses than conventional transformer designs.

- The base-case ultrastable designs have much higher HTS material requirements than the cryostable designs because of the large operating safety factor used in base case ultrastable designs. Decreasing the required operating safety factor (possibly through the use of external current limiting devices) would result in significantly smaller, more efficient ultrastable designs.

- The cryostability design metal stabiliz requirements $(64 \%$ to $88 \%$ of the winding weights for the base case designs) increase at roug $y$ the $4 / 3$ power of the current. Thus, transformers with larger ratings (and currents) will requil a higher percentage of stabilizer, so eddy currents will typically represent a larger fraction of $t:$ total losses. 


\subsection{System Integration Considerations}

In addition to performance, it is important to consider the system integration issues that apply when a new technology component, such as an HTS transformer, is added to a system built from components of a previous technology. The interfaces between the conventional and new technology parts of the system may require special engineering, equipment, and operating procedures to ensure that the whole system functions properly.

A primary purpose for considering these issues in a scoping study of this type is to review possible benefits that the new technology may bring to the system, as well as to highlight any special concerns or serious problems that might otherwise be overlooked. This chapter reviews a number of system integration issues to gain a first-order perspective on their potential to reinforce or modify the estimates of HTS transformer performance, as well as cost and market potential, which are presented in other shapters of this report.

\subsection{Basic Assumptions}

Any HTS component applied to an electric utility system can be anticipated to require some level of integration technology to accommodate the inherent differences between superconducting and conventional equipment. To help bound the number of integration issues involved, the following assumptions are made. The principal assumption is that future HTS transformers will be developed to the status of a "black-box" equivalent to a conventional unit with the same electrical characteristics. Thus, the rest of the system would "see" an HTS transformer, with appropriate internal electrical design, as just another transformer. Using similar judgments as those currently applied in procuring equipment from competitive vendors, utilities would purchase HTS transformers as unit-for-unit alternatives to conventional units.

The above assumptions probably apply more to transformers and other discrete circuit components than to HTS generators and transmission lines. This judgment is based on the potential of the latter to provide significant and cost-effective changes in utility system design in addition to performance and cost advantages at the component level. As an example, future HTS generators may be developed to deliver power directly at transmission line voltages. This development would eliminate the step-up transformer interface and, therefore, provide a potentially significant additional operational and cost advantage. Such a revolutionary change in the configuration of the system would require consideration of system-wide impacts and integration issues. In contrast, the discrete function of transformers allows the consideration of integration issues to be localized at their points of connection to the system.

\subsection{Advantages and Benefits of HTS Transformers}

If the enabling materials technology is developed, analyses in other chapters of this report show that HTS power transformers could be made significantly more efficient, smaller, and lower in weight and life-cycle cost than conventional units of the same capacity. These attributes translate into a 
number of system benefits and operational advantages. While utilities could take direct advantage of the higher efficiencies and lower costs of HTS transformers as aspects of a least-cost service strategy, these transformers have physical characteristics that offer other system-level values.

Superconductive transformers are projected to be smaller and lighter than conventional units at a given capacity. Depending on capacity and design efficiency, size and weight reduction by factors ranging between 3 and 6 appear possible (see Section 3.6). This expectation has been reinforced by recent Japanese research on a 72-kVA low- $T_{0}$ superconductive transformer that weighed $24 \mathrm{~kg}$ without cooling system and tank (Funaki et al. 1988). The authors estimated that this represented a 7:1 weight reduction ratio when compared with an equivalent conventional unit.

The size and weight reduction potential of HTS transformers could result in lower transportation costs and easier logistics when new units are delivered or when failed units need replacement. These weight and size advantages are, of course, partially offset by the additional refrigeration required. However, large HTS transformers are capable of being shipped in a number of pieces, the largest of which could be much smaller than an equivalent conventional transformer. In this respect, an early application of HTS transformers might be as spare, truck-transportable units protecting banks of heavier conventional units. Replacement of such units would otherwise involve slow-moving transportation by railroad or heavy-duty highway equipment. Such a rapid-response capability could reduce the number of spares needed throughout the system ior a given level of protection.

The HTS transformers could embody higher capacities than conventional units of a given size and weight, an attribute that offers a means of expanding the capacity of facilities that might otherwise be limited. For example, a common utility need is to increase substation capacity as load growth occurs. In many locations, especially urban sites, substations reach limiting capacities and cannot be expanded with conventional equipment. The solution is often to construct a new substation elsewhere, sometimes even further away from the load. In these cases, the installation of HTS transformers and other superconductive equipment such as current limiters, fuses, switches and busbars, might increase the capacity of the substation site, thus offering a cost-effective and preferred alternative.

The foregoing potential advantages and benefits of HTS transformers are supported by the results of analyses presented in other sections of this report. These analyses, however, do not address issues that may represent system integration problems or barriers to utility acceptance of the technology. The most important of these issues are discussed below.

\subsection{System Integration Issues Considered}

This review of system integration issues is based on the expectation that an HTS transformer can be designed to be electrically equivalent to a conventional unit of the same capacity. This means that the electrical performance specifications, including capacity, primary and secondary voltages and currents, design impedance and insulation levels, would be the same for an HTS unit as for an equivalent conventional transformer. The transformer design code developed in this project operates on this principle. The system integration issues discussed in this section relate to the following topics that are not considered in the code: 
utility acceptance of the refrigeration subsystem

- protection of the cryogenic subsystem

- protection against system faults and overloads

- operation and maintenance

- environmental issues.

\subsubsection{Utility Acceptance of the Refrigeration Subsystem}

Looking as far into the future as may be reasonable, a primary expectation is that all superconductive systems will need refrigeration. This appears to be a defensible projection unless HTS materials technology makes a remarkable breakthrough in the direction of achieving so-called "room-temperature" superconductivity. For electrical power equipment, even "room-temperature" HTS materials would need to be superconductive between $100 \mathrm{~K}$ and $200 \mathrm{~K}$ above ambient temperature to allow heat transfer to the environment by conventional, nonrefrigerant fluid circulation. As the prospect for this is remote, refrigeration appears to be an inseparable element of HTS power equipment.

There are two basic options in the choice of a refrigeration subsystem for HTS transformers of the future. The first would create the refrigerant supply on-site, at the location of the transformer. The second option is to produce the refrigerant at a central bulk-production plant and deliver it by truck, or possibly by pipeline if the distance is short, to an insulated storage container (dewar) at the transformer site.

Many fluids and refrigeration approaches combine to provide refrigeration over a continuum of temperatures ranging from those of liquid-helium up to near-ambient temperatures (Weast 1983; Dodge 1944). It appears, therefore, that existing refrigeration techniques can accommodate any desired operating temperature needed by any likely superconductor technology of the future. While refrigeration is a mature technology today, new developments such as systems based on the Stirling cycle (Ross 1988) offer the potential for further improving COPs and reliability levels. Such improved systems will probably be available by the time HTS applications reach the field demonstration stage.

A not so straightforward issue is whether the utility industry will conclude that the added complexity, cost, and maintenance requirements of refrigeration equipment are adequately compensated for by the advantages of HTS transformers. A typical utility might question the reliability of refrigeration and the possibility that refrigeration equipment might require inspection and service more often than a conventional transformer. Utility management also may not have maintenance staff with appropriate skills or may not wish to hire and train new personnel specializing in refrigeration.

Reasons such as these could become critical barriers to utility acceptance of HTS technology, regardless of the maturity level of the technology itself. However, alternatives appear to be available that can mitigate these concerns. First, there would be strong incentives for manufacturers to 
engineer on-site refrigerant systems specifically for the application. Driven by market requirements, refrigeration for HTS equipment should eventually achieve a level of reliability commensurate with that of conventional utility equipment.

Alternatively, the potentially simpler system of transporting the refrigerant fr production plant would eliminate the need for a local, possibly complex, refrigeratin. HTS transformer site. It is conceivable that simple, once-through cooling provide $\because$

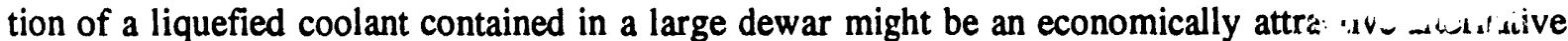
with low technological risk. With refrigerant supplied from a large dewar at the site, the increased COP achieved by large-scale production at a bulk liquefaction plant could compensate for the cosi of transporting the liquid to the site.

As a final possibility, the entire refrigeration subsystem could be operated and maintained by a company specializing in liquefied gas or refrigeration service. The vendor might, in addition, own the equipment and provide service contracted to the level of reliability required by the utility. Onsite, vendor-owned plants supplying liquid-gas cryogens are widely used by a number of different industries including manufacturers of chemicals, steel products, freeze-dried food, and electronic components. Such practice is well established in these industries, many of which have requirements for refrigeration today that are as stringent as any might be in the future case of an electric utility operating HTS equipment (Liquid Air Corporation 1987).

In consideration of the above, refrigeration, even as it exists today, would not be a technulogical barrier and could be installed on HTS power equipment wherever it might be located in the system. This applies to transformers located at manned, unmanned, and/or remote sites. Options and alternatives abound that could enable refrigeration to $r$ thus negating their concerns.

\subsubsection{Protection of the Cryogenic Subsystem}

In a conventional transformer during normal operations, internal temperature is the principal real-time variable that must be managed. Generally, as temperature changes in response to the electrical load on the unit, thermostatically-controlled cooling of the transformer keeps the unit within acceptable operating limits. Other changes in the status of the internal components of the unit (for example, evidence of insulation degradation or thermal decomposition of the oil) are typically monitored during periodic inspections.

In contrast, an HTS transformer would need a means of maintaining a dynamic equilibrium of internal conditions including such variables as coil voltage, current, impedance, temperature and mechanical stress, coolant flow rate, and temperatures inside and outside the cryostat. These parameters would need to be continuously monitored and, if necessary, controlled to maintain the coils in a safe, superconductive state.

This real-time monitoring and control system (MCS) would be required to provide operating margins that prevent the coils and cryostat from approaching critical conditions, such as $J_{c}, T_{c}$, or the limiting heat transfer capability of the coolant. The MCS would be needed to track and anticipate other variations such as aging effects, erosion or breakage of superconductive filaments, coolant 
contamination, and incipient blockage of coolant flow passages, all of which could lead to instabilities or eventual unit failure. Sensing and control of cryogen flow rates would be necessary for loadfollowing, cooldown, warm-up and transient management. MCS control of the refrigeration subsystem and communication with the utility's supervisory control and data acquisition (SCADA) system would also he essential in HTS transformers.

Therefore, an unavoidable extra-cost item (in comparison with conventional transformers) would be the need for HTS transformers to incorporate an active, on-board MCS connected to an array of internal sensors. The MCS in an HTS transformer should incorporate a microprocessor that integrates and responds to inputs received from sensors placed throughout the unit (both inside and outside the cryostat enclosures), as well as providing information on ambient conditions and, possibly, the status of the external transmission system. While a microprocessor-based control system could be expected to manage internal drift, aging, and responses to loads within the rated range, some elements of stability control would probably remain at the system connection interface, as discussed in Section 4.3.3.

The need for an MCS in each HTS transformer might be considered another acceptance issue similar to that of refrigeration, as discussed above. However, utilities are already comfortable with computers and complex electronic systems such as SCADA. The need for an MCS on HTS components should, therefore, not be a future "culture shock" or an acceptance issue with the utilities. One other mitigating effect may be that the differential cost contributed by the MCS could be less than currently foreseen if, as may happen in the future, utilities begin to equip conventional transformers and other components with real-time monitoring systems. Nevertheless, any MCS applied to a conventional unit would probably never employ the sophistication of such units needed for HTS transformers.

\subsubsection{Protection Against System Faults and Overloads}

The anticipation that HTS transformers are "black-box" equivalents of conventional units means that much of the standard protection equipment, such as fuses, relays, circuit breakers, and surge arresters, would be applicable to both types of unit. This is to be expected because in both an HTS transformer and a convention: 1 unit some protection is inherent in the chosen design impedance. It may also be possible to engineer large safety margins and thermal inertia into the cryosystems of HTS transformers to provide higher levels of automatic protection against fault currents and surges transmitted by the system. However, the sensitivity of an HTS transformer to system faults and overcurrents would be different and would require different protection strategies, depending on the philosophy of the cryostability (cryostable versus ultrastable) design.

In units designed to be cryostable, which incorporate a stabilizer, startup inrush currents, which might instantaneously reach up to 20 times rated load current (and fault currents of this magnitude) would instantaneously quench the coil system and be conducted primarily through the stabilizer. However, for typical overcurrents and surges that last only a few cycles at power frequency, the amount of heat energy generated by current flow in the stabilizer could be rapidly absorbed by the coolant and refrigeration system. The transformer would reestablish superconductive operation after the current transients subside. With appropriate design of the cooling system and sufficient margin 
between coil operating temperatures and the $T_{c}$ of the superconductor, the cryostable HTS transformer could conceivably rely on totally conventional protection equipment.

In the ultrastable concept, the use of redundant HTS windings reduces the current density to onetwentieth of $\mathrm{J}_{c}$. The chance that such a coil system would be quenched by current excursions during normal operations is negligible. The local failure of an HTS filament in a conductor matrix resulting from local mechanical stress or fatigue could be compensated by redistributing its current into parallel filaments. This level of redundancy could automatically handle the smaller current transients on the system. The ultrastable coil would, nevertheless, risk being quenched by the larger faults or overcurrents caused, for example, by some lighting strikes.

Because the ultrastable transformer has no stability metal, a large overcurrent that causes a quench could also damage the unit. Therefore, ultrastable units would need to be equipped with fast-acting (probably a fraction of a cycle) current limiters or circuit breakers that inhibit all current flow above a safe level. Special circuit breakers, ramp-up regimens, or other means of external current control would be needed to limit current to a safe level. The maximum safe current would probably need to be somewhat lower than the nominal overcurrent capacity of the coil to provide a margin that would allow for any aging or other deterioration during its lifetime.

Some very detailed analysis and hardware evaluation will be needed to develop the internal design of HTS transformers so that control stability issues and dynamic response under operational conditions can be predicted. Such information would provide necessary programming for the MCS and would guide the selection of protection and control equipment located at the system interface.

An important focus of such analysis should be the transient response of the coil/cryostat subsystem during the time between an event and the activation of the protection system. However, this issue may decrease in importance as smart solid-state switching devices are applied to electric transmission systems during the next 20 years. The Electric Power Research Institute predicts that high-power electronic devices based on the thyristor will replace present current limiters, circuit breakers, and other protection equipment (EPRI 1989). The operating speed of a thyristor-based circuit breaker is expected to be some 60 times faster than today's devices. Current could possibly be interrupted within $0.5 \mathrm{~ms}$ after the thyristor-based circuit breaker receives the signal, compared with the 30-ms response time of present mechanical circuit breakers. If high-power thyristor switches, circuit breakers, and current limiters become reality, the issue of control speed and transient management may become moot with respect to HTS transformers.

\subsubsection{Operation and Maintenance}

A conventional transformer can be energized, loaded, or deenergized instantaneously and essentially at will. Perhaps the bigger operational contrast between conventional and HTS units would be the latter's need to go through a long (probably several hours) and energy-intensive first cooldown before any useful operation is achieved. A similarly protracted warm-up period would be necessary to facilitate most maintenance that might be needed inside the cryostat, although other maintenance could conceivably be performed while the unit was cold. 
Utilities operating HTS transformers would most likely try to minimize the number of thermal cycles and would find it preferable to keep the unit in cold standby status when not electrically energized. The HTS transformer design could accommodate maintenance of the noncryogenic parts while the cryostat is cold. This would allow monitoring and sampling of oil, testing and maintenance of auxiliaries, and general inspection activities without bringing the cryostat to ambient temperature. However, the whole unit would need to be warmed up to ambient temperature to facilitate items such as maintenance and repair of coils, cryostat enclosure, leads, and sensors. Overall, an HTS unit should require less frequent on-site inspection because its general condition and need for maintenance and repair could be interrogated remotely through its SCADA-linked MCS. However, a similar capability would also be possible on a simpler scale with conventional transformers.

Maintenance of the refrigeration system for HTS transformers would follow conventional practice. In cases when the refrigeration unit could need extensive service, the transformer might still be kept cold and even in full-load operation with a truck-delivered supply of liquid cryogen.

The presence of the cryostat could complicate the functioning and maintenance of typical transformer accessories. For example, some difficulty might be encountered in engineering a load tap change mechanism because direct mechanical connection to the coil windings is required. Leads passing through the wall of the cryostat from each tap point might create a prohibitive heat leak, and the mechanical integrity of the connections might be difficult to maintain. HTS transformers could require the addition of external regulation equipment to be equivalent in capability to some conventional units.

The above represents a first-level sampling of possible differences in maintenance and operations between conventional and future HTS transformers. While it appears there are alternative approaches to these issues, it is also clear that utilities would have to adopt some new operational, inspection, and maintenance regimens for HTS transformers if they were to be put into system use.

\subsubsection{Environmental Issues}

At a first level of consideration, there appear to be no environmental issues of any great significance that would impede the use of HTS transformers. The use of refrigeration equipment is the principal difference between HTS transformers and conventional units. The risk of harm from the release of the cryogen is minimal. The widespread use of refrigeration and cryogenic facilities is based on well-established and accepted technology.

The coolant most likely to be used [liquid nitrogen $\left(\mathrm{LN}_{2}\right)$ ] is environmentally benign. Releases of $\mathrm{LN}_{2}$ could be a potential hazard in a confined space such as a transformer vault where the risk to personnel might be asphyxiation or cryogenic burns. Generally, these risks are encountered and managed in other applications by appropriate operating procedures, together with training and supervision of personnel. The use of other cryogens and coolants could raise safety, health, and environmental issues on a case-by-case basis. However, such speculation is unwarranted, at present, when the direction of HTS technology evolution is still so unclear.

The electric and magnetic field effects of electrical equipment are a growing concern that embraces components of electric utility systems as well as electric appliances in the home and 
workplace. While the external fields created by individual HTS transformers would be conditioned by their specific configuration, it is expected that superconducting transformers, in general, would generate external field intensity similar to conventional transformers with equivalent capacity. If field effects were to become a significant issue with regard to transformer design, HTS transformers could have an advantage over conventional units because of their tendency to be smaller. This would allow the design choice of lower intensity leakage fields at a given distance from the unit or, alternatively, a smaller, less costly structure to provide any shielding that might be necessary.

Some attributes of the HTS transformer might actually offer environmental advantages over conventional units. Because they are designed to handle no-load losses only, fans and pumps for the ambient-temperature core-cooling system handle a smaller load on HTS transformers than that of the equivalent conventional unit. This, coupled with HTS units being generally smaller, could result in a reduction in the audible noise being generated by each unit. Even though the HTS transformer requires ancillary equipment such as the refrigerator, the size and weight of the main tank can be reduced with an HTS design. Transformers that are physically smaller could have certain social values; for example, permitting more compact substation design or causing less obstruction to traffic during transportation. Ancillary equipment, including the bushings and cooling system components, would be shipped separately and assembled on site.

\subsection{Summary Comments}

This study concludes that, in common with other self-contained devices such as current limiters, switches, fuses and busbars, future HTS transformers could be installed on electric utility systems as direct substitutions for conventional equipment. As discussed above, the HTS transformer does not appear to present unsurmountable problems in installation and operation, although some clever design and engineering will be required in some areas. In addition, utilities would need to adopt somewhat different operations and maintenance procedures to accommodate the different functional nature of cryogenic equipment compared with conventional equipment.

Market acceptance of a new technology is not based solely on technical performance and economic issues. Utility acceptance of HTS transformers, even after they might be considered to be fully engineered, will probably be conditioned heavily by factors such as incompletely demonstrated reliability, lack of user experience, low confidence in a new technology and, in general, management conservatism. The utility industry has historically dealt with such issues with extreme caution and has required extensive field testing and evaluation of hardware to resolve these reservations. 


\subsection{Cost Characterization}

This chapter describes how transformer cost estimates were developed and provides summary results for transformer capital costs, operating costs, and life-cycle costs. Section 5.1 provides a general description of the transformer cost analysis objectives, principal problems associated with developing the cost estimates, and some discussion of uncertainties associated with the estimates. Section 5.2 describes the approach taken in developing the cost estimates. Data sources and results for transformer capital and operating and maintenance costs are contained in Section 5.3, along with results of the life-cycle cost analysis. Finally, Section 5.4 provides a short summary of some of the key conclusions from the cost characterization.

\subsection{Development of Cost Estimates}

The objective of the analyses described in this chapter is to develop comparable life-cycle cost estimates for HTS transformers and other types of transformers. The life-cycle cost represents one of the key factors that drives the market acceptance of a new technology. Life-cycle costs represent the present value of all expenses associated with owning an item over the item's life. These expenses include initial purchase and installation costs, annual operating and maintenance (O\&M) costs, and energy costs (a special type of O\&M expense). Assuming that two alternative technologies can perform the same service (i.e., they are designed such that each meets equivalent technical specifications for accomplishing a job), the technology that has the lowest life-cycle cost is the best choice from an economic standpoint.

Because the purpose of this activity is to develop an understanding of how the economic attractiveness of HTS transformers is likely to compare with other transformer designs, it is most important to understand how the life-cycle cost estimates compare in a relative sense. Therefore, the importance of the relative transformer comparisons was a major factor in developing the approach for the cost estimates. More emphasis was placed on developing cost estimates for items that are likely to vary among transformer technologies than was placed on items that tend to impact all types of transformers similarly.

Definitive estimates of HTS transformer costs cannot be produced at this time. The most difficult problem and source of the largest uncertainties is associated with the capital cost of components using HTS materials. Ongoing research to develop improved HTS materials continues, but the final properties and costs of the best materials for engineering application are still highly uncertain. The approach developed and used in this study for estimating costs has attempted to minimize the final uncertainty by estimating cost impacts on components that can be characterized (such as refrigeration) and making assumptions (guided by engineering judgment) only on equipment that is intrinsically unknown. Sensitivity analyses have been conducted to indicate the potential impact of significant changes in key assumptions.

The Study Advisory Group (SAG), described in Appendix A, contributed significantly to the analyses described in this chapter. Some of the key interactions with the SAG included obtaining historical data on conventional transformer purchase costs, insight on the projected future 
developments of amorphous core transformers, and information on past and current utility practices for evaluating and selecting transformers. Of particular importance was insight provided by transformer designers on the relative impact of various transformer components on the total capital cost of the transformer. This information was crucial in evaluating the impacts of HTS windings and amorphous cores on transformers that use those components. Information and data received from the SAG were evaluated for applicability to this study's purpose and, in some cases, were substantially revised prior to use.

The process of developing the life-cycle costs produces a number of results, including a breakdown of capital costs, estimated O\&M expenses, estimated values of the annual losses from transformer load and no-load losses, and present value calculations for each of these costs. In interpreting these results, the reader should keep in mind that they were developed so that technologies could be compared on a consistent basis. For example, the capital cost estimate of a specific conventional transformer does not represent any specific transformer purchased in the past. Nor does it represent the lowest expected capital cost for a transformer of that rating. What it does represent is the most economically effective design from a life-cycle cost perspective for a consistent design and cost methodology applied with a specific set of economic assumptions. The best and most appropriate use of the cost results is to allow comparisons among the different transformer designs analyzed in this study.

An interesting question in interpreting the results of the life-cycle cost analysis is the overall level of uncertainty in the results. While this is a general concern for the evaluation of any new technology, it is particularly true in the case of HTS equipment, where much of the fundamental design information for the HTS materials is currently speculative.

It is helpful to consider the uncertainty in terms of the absolute and the relative uncertainty. For example, installation costs for all of the transformers evaluated are estimated using the same cost estimating relation regardless of transformer type: uncertainties in the transformer installation cost will have an impact on whether or not the life-cycle cost of an individual transformer is correct in an absolute sense, but will have little effect on the relative comparison between two types of transformers. On the other hand, the estimated costs of the cryogenic refrigeration system do not impact all transformers types equally: uncertainties in the cost of the cryogenic refrigerator will create both absolute and relative uncertainties. Relative uncertainties may have a significant impact on the study results, and so are addressed in Chapter 6, Sensitivity Analyses. While the absolute uncertainty has been kept as low as was reasonable, this uncertainty does not affect comparison among transformer types and so was not explicitly addressed in any sensitivity analysis.

\subsection{Approach Used}

The approach used in developing the life-cycle costs for the transformers was driven by two needs: the need to be able to compare different transformers and the need to be able to evaluate transformer design tradeoffs. To ensure comparability, the methods, assumptions, and data were developed that would apply to all of the transformers analyzed. For instance, costs for conventional transformers varied widely depending on the specific application. It would not be possible to make a 
reasonable comparison of the costs for a HTS transformer and a conventional transformer unless both were designed for the identical application.

The variation in conventional transformer costs also highlighted the need to be able to evaluate transformer design tradeoffs. As discussed in Chapter 2, it is possible (within limits) for any transformer design to reduce energy losses by increasing the quantity of transformer materials. This creates a design tradeoff: costs for energy losses may be reduced at the expense of increasing the transformer capital cost. The ability to evaluate the cost of a wide range of design configurations and to identify the design with the lowest life-cycle cust was essential for comparing transformers.

Given the large number of designs to be analyzed, the life-cycle cost calculations were computerized. The computer code required its applicability to all types of transformers evaluated and enough flexibility to handle the design tradeoffs considered. To focus project resources on analysis rather than on code development, we assessed the feasibility of adapting existing generic codes to the needs of the transformer life-cycle cost evaluation.

Two existing models were subsequently adapted for the project, and a third model was developed that integrated output from the existing models and performed the actual life-cycle cost analysis. The models used in the evaluation were

- FAST-E: Originally developed with DOE support, FAST-E is a generic model that uses a parametric cost approach to estimate the costs of equipment. Because FAST-E is designed specifically to support the development of cost estimates for advanced technologies where design information is limited, it has a number of advantages for applications to HTS transformers. FAST-E was used to estimate capital costs for certain portions of different transformer designs. The results from the FAST-E analyses were then evaluated in regression analyses to develop specific cost algorithms that could be incorporated in the integration model, EAST (discussed below).

- TEAM: The Economic Analysis Model (TEAM) is a financial analysis model developed at PNL to support evaluations of the economic feasibility of thermal energy storage systems, but with a generic structure so that it could be applied to other types of projects. TEAM has the following capabilities:

- Generates projections of the cash flows associated with constructing and operating an energy system, and calculates a variety of economic "figures-of-merit" to help determine the economic feasibility of the system.

- Can perform detailed financial evaluations of projects for well-specified designs, but is flexible enough to also allow easy use for preliminary evaluations of projects with only conceptual cost and performance data.

- Can quickly perform sensitivity analyses on the key project uncertainties (either performance or cost) to address the impact of these parameters on project economics. 
- Was used to calculate the life-cycle cost impacts for capital, O\&M, and energy values based on the economic assumptions developed for this study. Life-cycle cost factors developed using TEAM were then provided as inputs to the EAST model.

The TEAM model is documented in detail elsewhere (Brown et al. 1987).

- EAST: The Economic Analysis of Superconducting Technologies (EAST) model integrates the information from the design and cost models. FAST contains transformer cost algorithms that were developed from the FAST-E model and from other approaches. Each cost algorithm provides an initial cost estimate for a transformer element (such as the core) based on physical design parameters (such as core weight) provided by the transformer design code. After calculating all transformer cost elements, EAST then calculates the life-cycle cost of the transformer using factors from the TEAM code.

EAST is designed to aid in selecting the transformer design that results in the lowest life-cycle cost. For a given transformer type, EAST reads in a number (typically several hundred) of alternative transformer designs from the output files of the design code. The life-cycle cost of each design is evaluated, and the designs are then sorted in order of increasing life-cycle cost.

One of the advantages of the EAST model is its ability to perform sensitivity analyses quickly to determine the true impact of assumptions on the life-cycle cost. For example, consider an HTS transformer design where $30 \%$ of the life-cycle cost is due to the cost of superconducting materials. If the cost of the HTS materials is assumed to double, a simplistic analysis would suggest that the life-cycle cost of the transformer would increase by an additional 30\%. Such an analysis would ignore the existence of alternative transformer designs that would be more economically effective if the cost of HTS materials changed substantially. EAST provides the ability to investigate design tradeoffs easily, as well as more realistic answers to "what if" questions.

Information on the approach embodied in the EAST mudel for cost calculations is described in the following subsection. A more detailed description of the EAST model and data is contained in Appendix C.

\subsubsection{Capital Cost Approach}

Cost models expressing capital cost as a function of the transformer design parameters (e.g., core weight, windings weight) were used in the EAST code to analyze the thousands of alternative transformer designs considered. (Note: It is important to keep in mind that information in this section focuses on how the cost models vary among technologies, and not on how the estimated cost results vary.) Although the cost model may be identical between two technologies, the estimated costs produced with the model may vary substantially because of design differences between the technologies. For example, although the shipping cost model was identical for the conventional and HTS ultrastable designs, the model generally estimated lower costs for the ultrastable designs because of their lower weight.

Transformer capital costs are defined as the cost to the utility of initially acquiring the transformer. These costs include the purchase price, shipping costs, and installation charges. All of the 
transformer capital cost estimates assume the same level of maturity for each of the technologies. This eliminates differences that would arise in the technology comparisons because of initial development costs, startup costs, risk premiums embedded in the transformer sales prices, initial learning, and other factors that may increase the prices of new technologies during the early years of the product production. The capital cost estimates therefore represent long-run estimates of how the capital costs would compare at similar production levels and similar levels of development. Sensitivity analyses address how the purchase price of the HTS transformers and amorphous core transformers are likely to vary during the early years of production; this information is used in the market evaluation to estimate sales potential by year for each of the transformer types.

To be able to distinguish among the alternative transformer types, it is necessary to estimate how changes in the transformer components (such as changing from conventional windings to HTS windings) would affect the transformer purchase cost. The cost breakdown used to estimate transformer capital costs is shown in Table 5.1; the transformer shell, core, windings, cryocooler, and shipping represent a breakdown of the transformer purchase cost. Table 5.1 also summarizes how the cost models vary among technologies for each of the components. The approach used to develop each of the capital cost models is discussed below.

The core, windings, and shell represent the principal components of the transformer. Cost models for each of these elements were developed using the FAST-E computer package. The general method for each of these components was to obtain cost and design data for conventional transformers and use this data with the FAST-E code to develop a calibrated model of the cost of the compcnent as a function of weight. In developing the FAST-E models for conventional transformers, insight on the general cost contributions of each of these components to the transformer sales price was obtained from members of the SAG and from a subcontract to Kaiser Engineers.

Table 5.1. Transfer Cost Elements

\begin{tabular}{|c|c|c|c|}
\hline Item & Conventional & HTS-Cryo & HTS-US \\
\hline Shell & c & $\mathrm{h}$ & $\mathrm{h}$ \\
\hline Core & $\mathbf{s}, \mathbf{a}$ & $\mathrm{s}, \mathrm{a}$ & $\mathbf{s}, \mathbf{a}$ \\
\hline Windings & c & $\mathbf{x}$ & $\mathrm{h}$ \\
\hline Cryocooler & NA & $\mathrm{h}$ & $\mathrm{h}$ \\
\hline Shipping & c & c & c \\
\hline Installation & c & c & c \\
\hline
\end{tabular}

Key:

$c=$ conventional cost model

$\mathrm{s}=\mathrm{Si}$-steel core cost model

$\mathrm{a}=$ amorphous core cost model

$\mathrm{x}=$ high-temperature superconductivity composite cost model

$\mathrm{h}=$ high-temperature superconductivity cost model

NA $=$ not applicable. 


\subsubsection{Shell Cost Model}

As used in this analysis, the transformer shell represents both the structural container for the core and windings and also a general "miscellaneous" category to capture aspects of the transformer sales price not included in the windings and core components. The shell costs for the two HTS technologies were judged to be somewhat higher than the conventional shell because of the need to include cryogenic insulation in the HTS shells. The cost model for the HTS shell was developed by increasing the technology complexity factor in the FAST-E model. The HTS shell cost model is based on the design assumption of a solid rather than a vacuum insulation. Costs for vacuum insulation would be expected to be higher.

\subsubsection{Core Cost Model}

As identified in Table 5.1, two types of cost models were developed for the transformer core: a Si-steel core model and an amorphous core model. These models were independent of the technology type, since either core type was judged applicable for the HTS and conventional technologies. The Si-steel core cost model was developed as part of the FAST-E analysis of the conventional transformer. The primary sources of information on amorphous core cost trends were members of the SAG and contacts with amorphous core developers, although some general information was also obtained from the literature (EPRI Journal 1987).

While costs for amorphous core materials are currently much higher than for Si-steel cores, there was uniform consensus from all sources contacted that amorphous core materials will decrease significantly in price in the future. This will happen as additional manufacturing capacity comes on line, and as remaining problems in working with the more brittle amorphous core materials are overcome. The general opinion of the sources contacted was that the additional manufacturing difficulties of amorphous cores would place them at a cost premium (on a dollars per pound basis) relative to Si-steel cores. Based on discussions with developers, an initial materials cost multiplier of 1.5 was used with the FAST-E model for Si-steel cores to develop costs models for transformers with amorphous cores.

\subsubsection{Windings Cost Model}

The cost models of the HTS windings represent one of the key distinctions between HTS and conventional technologies, and so are discussed in some detail below. The fundamental variable needed to adjust the cost models of conventional transformer windings is a relative differential in the materials costs. There is obviously a high level of uncertainty associated with the cost of HTS materials caused by a lack of fundamental design information. The lack of specificity for HTS material composition and superconductor design dimensions, along with the lack of manufacturing experience with superconductors based on HTS materials, also contribute to the uncertainty.

A literature review was conducted to identify the cost assumptions used by other researchers in their analyses of superconducting systems. Vendors were then contacted to obtain both information on the costs of low-temperature superconducting (LTS) materials and their perspective on expected costs of HTS materials relative to LTS materials. The information gathered from the various sources was used to establish a nominal cost estimate for the base case assumption and a range of probable costs for sensitivity analyses. 
Reports prepared by Westinghouse (1982) and Argonne National Laboratory (1988) contained limited information on the costs of superconducting materials. The Westinghouse study looked at the application of LTSs in power transformers. Westinghouse estimated that niobium-based wire would cost \$150/lb (1982 \$). The Argonne study investigated the application of HTSs in several technologies, including power transformers, generators, transmission lines, and superconducting magnetic energy storage (SMES). Although it was noted that the bulk materials for 1-2-3 ceramic oxides cost only about $\$ 10 / \mathrm{lb}$, the individual technology analyses presumed a cost of $\$ 100$ to $\$ 200 / \mathrm{lb}$, apparently assuming the cost of HTSs would be similar to that estimated by Westinghouse for LTSs.

Several vendors were contacted for their opinions on the cost of LTS materials and composites, the key cost drivers for LTSs, and the expected relationship between LTS and HTS costs. Key information obtained from telephone conversations with representatives of each company late in 1988, representing data and price levels current at that time, is summarized here. Companies are designated as Vendors $\mathrm{A}, \mathrm{B}$, and $\mathrm{C}$.

Vendor A produces multifilament superconductors, primarily composites of niobium-titanium and copper. Key cost drivers were indicated to be the ratio of copper to superconducting material and the final superconducting strand diameter. Raw stock niobium-titanium was reported to cost about $\$ 70$ to $\$ 80 / \mathrm{lb}$, while the copper was only $\$ 2 / \mathrm{lb}$. Copper to superconducting material ratios of 3:1 to $6: 1$ were most common in their work. Delivered composites were usually under $\$ 100 / \mathrm{lb}$; most of their applications were in magnetic resonance imaging (MRI) systems. Although the raw materials cost for HTS materials was thought to be lower than for LTS materials, the fabricating costs are a complete unknown.

Vendor B is another producer of niobium-titanium/copper superconducting composites. The company representative indicated that raw stock niobium-titanium costs about $\$ 50 / \mathrm{lb}$. A simple monofilament conductor with a 4:1 copper to niobium-titanium ratio was estimated to cost about $\$ 50 / \mathrm{lb}$. In contrast, a conductor being developed for the Supercollider was expected to cost from $\$ 100$ to $\$ 150 / \mathrm{lb}$ because it employs very fine filaments $(5 \mu \mathrm{m})$ in a closely spaced arrangement. The representative expected the cost of ceramic oxide materials to be about the same as niobium-based stock. Processing costs for ceramic oxides were thought likely to be higher because of the general difficulties of fabricating ceramics compared with metals.

A representative of Vendor $C$ indicated that the cost of niobium-based superconductors varied by an order of magnitude depending on the design of the superconducting composite. Important design variables included the conductor diameter, copper/superconductor material ratio, number of filaments, filament pattern, and filament diameter. No generalizations of the typical cost per pound for LTSs or the expected costs for HTSs were offered. Representatives from two other suppliers (a wire drawing company and a supplier of niobium-titanium raw stock and a niobium/tin composite) echoed this vendor's opinion that costs depend too heavily on design specifications to quote a typical cost per pound.

In general, the niobium-titanium raw stock cost, copper/superconducting material ratio, and superconducting composite cost data reported by Vendor A and Vendor B are consistent with each other. The specific data reported by Vendor B implies a cost of about $\$ 170$ to $\$ 250 / \mathrm{lb}$ for fabricated niobium-titanium, if the cost of fabricated copper is assumed to range from $\$ 0$ to $\$ 20 / \mathrm{lb}$. A baseline 
value of $\$ 200 / \mathrm{lb}$ (1989 \$) of fabricated niobium-titanium was selected based on this range and the estimate of $\$ 150 / \mathrm{lb}$ prepared by Westinghouse.

HTSs were assumed to cost the same as LTSs as the result of mixed opinion as to their expected relative cost; all sources indicated it was a highly speculative question. Given the high level of uncertainty in the cost of fabricated HTS materials, a cost range was established around the nominal figure of $\$ 200 / \mathrm{lb}$ for sensitivity analyses. A cost of $\$ 50 / \mathrm{lb}$ was selected for the low end of the range based on the approximate cost of raw stock niobium-titanium. A cost of $\$ 1000 / \mathrm{lb}$ was selected for the high end of the range based on the implied cost of fabricated niobium-titanium in the composite conductor being developed by Vendor B for the Supercollider.

After the baseline assumption of the HTS materials cost had been developed, a FAST-E model of the HTS windings costs was developed by using a materials cost adjustment to the cost model developed for conventional windings. It should be noted that the cost model developed for the HTS windings was not a constant $\$ 200 / \mathrm{lb}$, which represents an input assumption to the FAST-E model. The HTS winding cost model varies the unit cost depending on the total amount of HTS windings required, with smaller transformers having greater winding costs per pound. For example, with small transformers the cost models developed with FAST-E predicted HTS winding costs of over $\$ 500 / \mathrm{lb}$, while for the largest transformer sizes the costs range near $\$ 250 / \mathrm{lb}$. The economies of scale represent unit cost savings in the manufacturing process rather than savings in the purchase price of materials.

For the HTS cryostable cases, the transformer windings are a composite of copper and niobiumtitanium superconducting materials. The total cost of windings predicted by the HTS cryostable model is determined by the total quantity of both types of materials in the composite design for a specific transformer. For the HTS ultrastable cases, the cost model is based on the use of a pure superconducting material.

\subsubsection{Cryogenic System Cost Model}

Costs for the cryocooler make up part of the transformer initial purchase cost for HTS transformers, but obviously do not apply to conventional transformers. A literature review of the purchase costs for cryogenic refrigerators identified little information aside from the often referenced paper of Strobridge (Strobridge 1974). Several vendors of liquid nitrogen cryogenic systems provided current purchase costs of systems at several capacity ratings. When adjusted to current price levels using a GNP deflator, the data from Strobridge were found to be in fairly good agreement with the current vendor data. Using both the Strobridge and vendor data, a regression analysis was used to develop cost of the cryogenic system as a function of capacity. Costs for integrating the cryogenic system with the HTS transformer were estimated as $40 \%$ of the cryogenic system cost, based on a review of typical installation costs for similar equipment (Guthrie 1974; Peters and Timmerhaus 1980).

\subsubsection{Costs and Price Changes}

Information on shipping and installation costs and price changes over time are explained in the following subsections. 


\subsubsection{Shipping Costs}

The cost models for transformer shipping were assumed to depend only on the transformer dimensions and weight and not on the transformer technology. Three scenarios were developed for estimating shipping costs. For each scenario a shipping distance of 1000 miles was assumed.

The first scenario applies to small transformers that could be shipped by truck (assumed to be shipments under $60,000 \mathrm{lb}$ ). Contacts with trucking companies indicated that $\$ 2 / \mathrm{mile}$ is a representative cost for these types of shipments.

The second shipping scenario applies to transformers weighing between 30 and 75 tons. These are assumed to be transported via rail to a location near the installation and then moved via multiwheel trailer to the installation site. Rail shipping costs based on railroad contacts were expected to be in the range of $\$ 5$ thousand to $\$ 14$ thousand. The major expense for this scenario is for the multiwheel trailer, which is a specialized trailer designed for the transport of oversize and overweight loads. The Bonneville Power Administration (BPA) recently estimated $\$ 600$ thousand to move a 200-ton spare transformer from one substation to another located 45 miles away (BPA 1988). Transformer shipment via multiwheel trailer was judged to be much less expensive than this estimate, because the BPA costs included removal of the spare from one location and installation at a new location. An assumption of $\$ 100$ thousand for the shipping cost was used for the second scenario, which is felt to be a conservative estimate (i.e., likely to be in the high range).

The third shipping scenario applies to transformers over 75 tons. The largest transformers in this range (400 tons) are assumed to be shipped by a special rail car (known as a Schnabel car) designed specifically for the transport of large transformers. Contacts with railroads indicated that a reasonable assumption for shipments by Schnabel car was $\$ 100 / \mathrm{mile}$, or $\$ 100$ thousand for the 1000 -mile shipping distance assumed. Adding $\$ 100$ thousand for the transport to the final site via multiwheel trailer brought the total shipping cost assumed for this scenario to $\$ 200$ thousand.

\subsubsection{Installation Costs}

Transformer installation costs are not expected to vary among technologies for similar designs. Literature reviewed provided little information on the installation costs for large transformers, other than that the installation costs would not be expected to vary significantly as a function of size (small or nonexistent economies of scale). The installation cost model developed was based on BPA information on total transformer cost relative to the purchase price (BPA 1988) and discussions with BPA field engineers.

\subsubsection{Adjustments to HTS Transformer Cost over Time}

Estimating market penetration during the early years of a new technology (see Chapter 7) requires an understanding of how the price of the product is likely to change over time. Because the life-cycle cost estimates of the HTS transformers were developed based upon long-term projections, it was necessary to modify these costs to estimate the annual sales potential as a function of time.

A number of factors influence how the price of a new technology varies over time. Typically the manufacturing costs of an item tend to decrease over time because of learning and experience in 


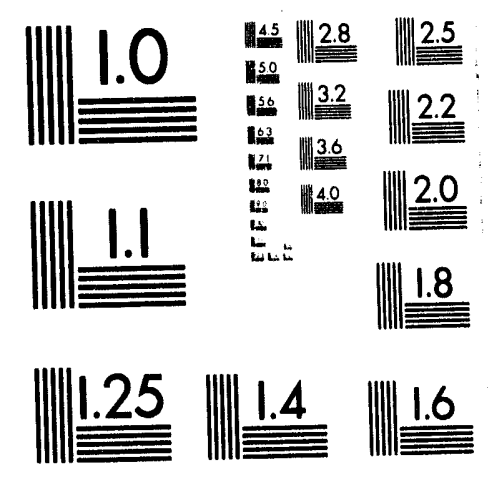




$$
\begin{aligned}
& \mathbf{N} \\
& \stackrel{O}{\omega}
\end{aligned}
$$


the manufacturing process, reductions in engineering/design costs, improvements in available materials, improvements in economies of scale as production level increases, and many other related factors. Along with these manufacturing cost trends are a number of strategic factors related to product pricing. Manufacturers must decide between pricing products at a high enough level tc recover initial development costs rapidly and pricing products at a low enough level to build a market position rapidly.

Developing comprehensive estimates of how HTS iransformer costs would vary over time is beyond the scope of the present study, and the uncertainties regarding ultimate materials properties would make such an effort subject to broad uncertainties at best. Accepting these limitations, our objective was to then postulate a simple, reasonable scenario for how costs would vary over time and determine if this had significant impacts on the overall results of the market analysis. In evaluatitig cost changes over time, we have assumed that conventional transformers have achieved their long-run price (by virtue of a number of years production) and have therefore focused only on how HTS transformer costs change over time. The rationale and assumptions made in developing the scenario for price reductions of HTS transformers over time are explained below.

Our basic approach in postulating early prices for HTS transformers was to work backward from the long-run cost, adjusting prices for components of the transformer that seemed most likely to vary because of the unique nature of the HTS design. Component adjustment factors were developed for the HTS materials, the transformer shell, the cryocooler, and an overall premium for the transformer pricing. Development of each of these adjustment factors is discussed below.

The cost for manufacturing transformers with HTS windings would be expected to vary significantly in early production years because of changes in materials prices (price would be expected to decline as production level increased), improvements in the winding design, and experience in working with HTS windings. We assumed that changes in the price for HTS materials would be driven by the overall demand for those materials, of which transformers would be a small part of the market. Making this assumption eliminates concerns regarding correlation of actual transformer sales to the annual price reduction for HTS materials; even if HTS transformer sales were zero in a given year, reductions in the price of HTS materials would still occur because of sales in other applications. For the purchase price of HTS materials, we assumed that the long-run cost would be obtained 10 years after transformer introduction, and that the cost would drop by $30 \%$ annually the first 5 years and $10 \%$ annually for the second 5 years. We assumed that the long-run engineering costs for the materials would be obtained in 5 years and would decrease by an average of $30 \%$ annually. Finally, for manufacturing learning effects, we assumed that the long-run productivity would be achieved in 5 years and that these effects would decrease the cost of manufactured windings by $20 \%$ annually. The sum of these materials adjustment factors is shown in Table 5.2. All materials adjustments were assumed to be minor after the tenth year of production.

HTS transformer shells are projected to cost more per unit mass than conventional transformer shells because of the cryostat's additional requirement for effective insulation. The cost of HTS transformer shells is expected to decrease over time because of reduced engineering/design costs and increased general manuıdcturing experience. We assumed that engineering costs would reach longnin levels in 2 years, with decreases of $30 \%$ annually during that period. For the manufacturing experience effects, we assumed the long-run values would be reached in 5 years with annual decreases of $20 \%$. 
Table 5.2. Adjustment Factors for HTS Materials

\begin{tabular}{cc} 
Year & Adiustment Factor \\
\hline-10 & 14.68 \\
-9 & 9.92 \\
-8 & 6.54 \\
-7 & 4.13 \\
-6 & 2.42 \\
-5 & 1.69 \\
-4 & 1.52 \\
-3 & 1.37 \\
-2 & 1.23 \\
-1 & 1.11 \\
0 & 1.00
\end{tabular}

Although cryogenic coolers at liquid nitrogen temperatures are a commercially available technology, costs for this component would also be expected to be higher than long-run values during the first years of transformer production. The primary reason for this is the need for initial engineering and design to integrate the cryogenic cooler into the overall transformer design. We assumed that these problems would be solved within a few years and that the long-run costs for the cryogenic cooler would be reached in the third year of production. We assumed cost decreases of $30 \%$ annually during early production.

The final adjustment factor used for the transformer was an overall "risk premium." The risk premium accounts for 1) the risks of providing warranties to custorners based on little production experience and 2) the requirements for increased amortization of production equipment in early years to reduce investment risk. The risk premium adjustment was applied to the total capital cost and was assumed to occur for the first 5 years of transformer production. We assumed an annual reduction of $15 \%$ for the risk premium.

The adjustment factors for the transformer shell, cryogenic cooler, and risk premium are summarized in Table 5.3. These factors and those for HTS materials were then applied to the long-term capital cost estimates of four HTS transformers to develop an estimate of how the transformer capital cost would decline over time. The transformers evaluated were an HTS-US autotransformer at $200 \mathrm{MVA}$, an HTS-CS autotransformer at 200 and $1000 \mathrm{MVA}$, and a step-up HTS-CS transformer at 630 MVA. The resulting scenario of HTS transformer cost reduction over time is shown in Figure 5.1. All of the cryostable designs had similar cost multipliers; the multipliers were roughly 3.5 times the long-term capital cost in the first year of production and declined to only $5 \%$ to $10 \%$ of the long-term cost after the fifth year of production. The ultrastable design has much higher initial cost multipliers than the cryostable designs because of the larger quantity of HTS materials used in the ultrastable design. In this scenario the ultrastable transformer would cost nearly a factor of 8 times more than the long-run cost in the first year of production. 
Table 5.3. Adjustment Factors for Transformer Shell, Cryocooler, and Risk Premium

\begin{tabular}{cccc} 
Year & Shell Total & & \multicolumn{2}{c}{ Cryocooler Engineering } & \\
& & & \\
-10 & 3.48 & 2.04 & 1.92 \\
-9 & 2.38 & 1.43 & 1.63 \\
-8 & 1.56 & 1.00 & 1.38 \\
-7 & 1.25 & 1.00 & 1.18 \\
-6 & 1.00 & 1.00 & 1.00 \\
-5 & 1.00 & 1.00 & 1.00 \\
-4 & 1.00 & 1.00 & 1.00 \\
-3 & 1.00 & 1.00 & 1.00 \\
-2 & 1.00 & 1.00 & 1.00 \\
-1 & 1.00 & 1.00 & 1.00 \\
0 & 1.00 & 1.00 & 1.00
\end{tabular}

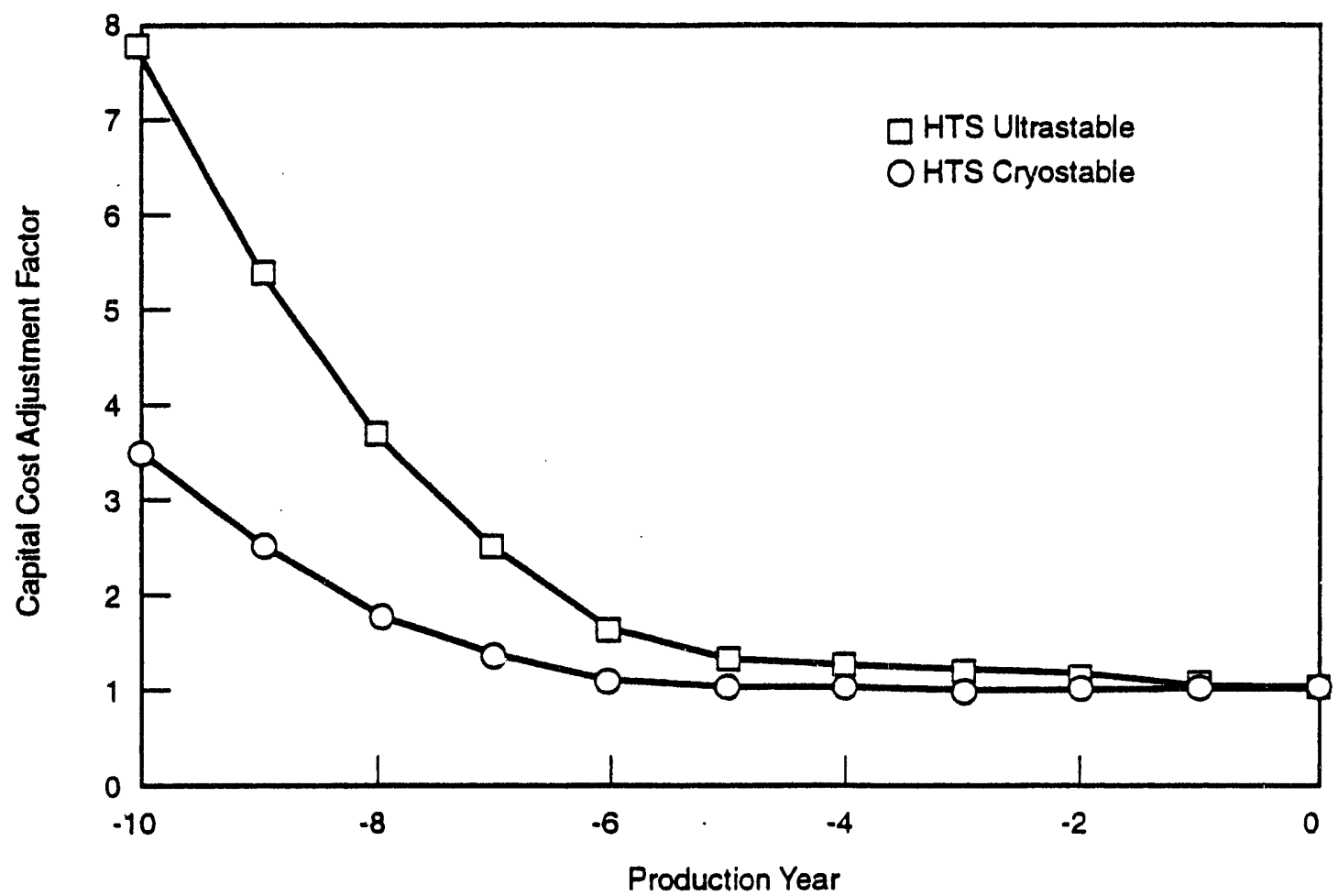

Figure 5.1. Capital Cost Adjustment Factors for HTS Transformers in Early Production Years 


\subsubsection{Cost Estimates for Operation and Maintenance}

With the exception of the cryogenic refrigeration system, the O\&M requirements for the HTS transformers were assumed to be similar to those for conventional transformers. This led to the development of two O\&M models: one for the cryogenic refrigerator of the HTS transformers and one for all other O\&M requirements. Because of a lack of specific data on transformers and cryogenic systems, maintenance costs for these items were estimated from rules-of-thumb relating annual maintenance cost to the initial installed capital cost.

Peters and Timmerhaus (1980) advise using $2 \%$ to $6 \%$ of initial capital cost for "simple chemical processes," while $2 \%$ to $20 \%$ of initial capital cost is presented as the range for all capital items. Happel and Jordan (1975) state that $2 \%$ to $3 \%$ of capital is common for ambient or near-ambient temperature processes with few moving parts. In the Electric Power Research Institute's (EPRI) Technical Assessment Guide (1986), the recommended annual maintenance rate for general facilities, steam, and electrical equipment is $1 \%$ to $3 \%$ of capital per year. Based on the above data, the annual maintenance costs for conventional transformers were set at $2 \%$ of initial capital cost. Maintenance costs for superconducting transformers were judged to be the same as conventional transformers because maintenance duties (physical inspections, oil filtering) are expected to be similar.

The cryogenic system, with its moving parts operating in a high-pressure and low-temperature environment, seems typical of average chemical process equipment, for which Peters and Timmerhaus (1980) and Happel and Jordan (1975) suggest 6\% of initial capital per year. A range of 3\% to more than $6 \%$ is given in EPRI (1986) for severe conditions characterized by solids handling, high pressure, or high temperature. Two other estimates were found for technologies similar to the cryogenic system. Happel and Jordan (1975) estimated 5\% of capital for compressors and Brown and Spanner (1988) estimated $4 \%$ to $6 \%$ of capital for residential air conditioning systems. Based on the above data, the annual maintenance costs for cryogenic systems were set at $5 \%$ of initial capital cost.

\subsubsection{Value of Energy Losses}

The value of energy losses from the transformer is an important variable that affects both the optimal design of the transformer and the total life-cycle cost. The appropriate value of energy to use in the transformer analysis is the present value of the energy losses expected to occur over the life of the transformer. Cost of energy naturally varies tremendously among utilities, depending on the type of generating resources they operate; energy cost varies also for a given utility, depending on the part of the system in which energy losses occur. In the actual practice of specifying and evaluating transformers, a utility may specify different values of energy losses depending on where the transformer is located and how it will be used. For this study, two types of applications were considered in developing assumptions for the value of energy losses: generating step-up transformers and autotransformers used in the transmission system (grid transformers).

A conventional step-up transformer uses energy only from the generating plant and is operated in the same duty cycle as the power plant in which it is installed. For HTS step-up transformers, we assumed that the transformer would stay at operating temperature even during plant downtime to 
eliminate startup time and reduce thermal cycling problems. This operating approach creates two types of energy use by HTS transformers: operating losses and downtime losses.

The appropriate value of operating energy losses for a step-up transformer is based on the present value of energy produced at the plant. For this study, step-up transformers are assumed to be installed at a baseload coal-fired power plant (630-MVA case), an intermediate load natural-gascombined cycle power plant (310-MVA case), and a natural-gas-fired peaking power plant (60-MVA case). The value of operating energy losses varied for each of these applications. Downtime losses (which apply only to HTS transformers) were assumed to be taken from baseload power provided by the utility grid.

Establishing baseline assumptions of energy values for transformers in the transmission and distribution (T\&D) system is much more complex. The energy for a grid transformer is supplied not from a single power plant, but from a mix of plants. The value of energy losses in this situation changes hour by hour throughout the year as the utility uses different power plants to meet the current load on the grid. An additional problem for grid transformers is that their loading changes substantially throughout the year. As the transformer load changes, the energy loss also varies. Energy loss values for transformers are generally characterized as either no-load losses or load losses, as discussed in Chapter 3. No-load losses (or core losses) are constant throughout the year and do not vary with the load, while load losses (or winding losses) vary according to the square of the transformer load. The production cost of energy for no-load losses was developed based on the assumption that this energy would be provided by a baseload coal-fired power plant. The production cost of energy for load losses was developed based on the assumption that this energy would be provided by a natural-gas-fired peaking power plant.

In addition to the energy production cost, energy loss values for grid transformers should include a consideration of costs related to power transmission from the power plant to the transformer and to reserve margins. Transmission increases the value of energy because of the energy losses in the transmission process, as well as the capital cost required for the transmission equipment. The need for utility reserve margins tends to have a multiplicative impact on the capital cost component of e'ergy production values. For example, using the $20 \%$ reserve margin assumed for this study, inc, sasing the load by $1 \mathrm{~kW}$ in a T\&D transformer requires $1.2 \mathrm{~kW}$ of additional capacity to maintain an identical reserve margin.

For this study, a value of $3 \%$ was assumed for energy losses in the transmission system. This value appeared reasonable in light of estimates for total T\&D losses (including distribution) of 7.5\% for the BPA grid, and 3.0\% to $15.5 \%$ for various utilities in California (Northwest Power Planning Council 1988; California Energy Commission 1985).

The estimated costs of energy production from the baseload, intermediate, and peaking plants were developed from the capital, and O\&M cost characteristics were obtained from the literature (EPRI 1986). Current and future fuel costs (cost to the utility) were established by reviewing projections published by EPRI (1986), the Energy Information Administration (1989), Data Resources, Inc. (1987), Wharton Econometric Forecasting Associates (1987), and the Gas Research Institute (1987). The life-cycle value of energy produced at the plants was calculated with the TEAM model using the same economic assumptions described in Section 5.2.5. 
The baseline energy values developed and used in the transformer evaluation are shown in Table 5.4 in dollars per kilowatt-hour $(\$ 1 / \mathrm{kWh})$ and dollars per kilowatt $(\$ / \mathrm{kW})$. The $\$ / \mathrm{kWh}$ unit shows the levelized energy cost (LEC) for the energy value. The LEC is defined as the annual energy cost which, over the lifetime of the application, would produce a present value equivalent to all the expenses associated with generating the energy. The LEC shown in Table 5.4 is expressed in real dollar terms, which factors out the effects of general inflation. The second unit shown is $\$ / \mathrm{kW}$, which is a typical utility figure used to express the values of losses for T\&D applications. The energy value in $\$ / \mathrm{kW}$ is based on the cost of producing the energy for a 30 -year transformer application and is also dependent on the transformer load factor assumed (which is why the term varies for the downtime losses of grid transformers).

Table 5.4. Values of Energy Losses (levelized energy cost, constant 1989 dollars)

$\begin{array}{lcc} & \$ / \mathrm{kWh} & \$ / \mathrm{kW} \\ \text { Step-Up Transformers } & & \\ \text { Peak plant operating losses } & 0.094 & 840 \\ \text { Peak plant downtime losses } & 0.066 & 5,330 \\ \text { Intermediate plant operating losses } & 0.059 & 1,590 \\ \text { Intermediate plant downtime losses } & 0.066 & 4,150 \\ \text { Baseload plant operating losses } & 0.055 & 3,180 \\ \text { Baseload plant downtime losses } & 0.066 & 2,070 \\ & & \\ \text { Autotransformers } & & \\ \text { No-load losses } & 0.066 & 5,920 \\ \text { Load losses } & 0.112 & 4,190\end{array}$

\subsubsection{Calculations of Life-Cycle Costs}

Life-cycle cost calculations are used for making choices between alternative investments when the initial costs, operating costs, and benefits of the investments vary. The life-cycle cost calculations provide a means to evaluate changes in the transformer efficiency (through economic analysis of the annual energy loss) and trade these off against the other transformer costs.

In this way, the life-cycle cost predicted for HTS applications represents a "bottom line" figure that indicates whether the HTS application will be economically competitive against other technologies. 
Utilities and industry use many alternative approaches and methods to determine the life-cycle cost of alternative investments. A fundamental principle underlying all approaches that can be considered economically correct (i.e., a principle that would maximize wealth over time when used as a decision basis) is that of the time value of money. The time value of money recognizes that the promise of money to be received in the future is less valuable than money already held; this leads to the concept of discounting future cash flows to an equivalent "present value." Beyond the fundamental principles encompassed in life-cycle cost calculations, methods vary tremendously in terms of the level of detail used (i.e., which types of simplifying assumptions are made), and the types of economic assumptions (such as discount and inflation rates) used. The level of detail and economic assumptions will generally have a significant impact on the life-cycle cost values calculated.

The life-cycle cost calculations in this study are all based on an after-tax cost of ownership. The calculations are developed with an economic evaluation model previously developed at PNL, which is described in more detail elsewhere (Brown et al. 1987). General economic assumptions used in the life-cycle cost calculations are based on an investor-owned utility for the HTS application, and are summarized in Table 5.5. The basic approach to life-cycle costs used in this study is to calculate the present values of all costs associated with owning the transformer. This approach is sometimes called calculation of a "total owning cost" (TOC). The figure of merit TOC can be calculated as

$$
\text { TOC }=\text { PV }(\text { Capital })+P V(O \& M)+P V(\text { Energy Loss })
$$

where

$$
\begin{aligned}
\text { PV(Capital) } & =\text { present value of transformer purchase cost } \\
\text { PV(O\&M) } & =\text { present value of transformer O\&M expense over transformer life } \\
\text { PV(Energy Loss }) & =\text { present value of transformer energy losses over transformer life. }
\end{aligned}
$$

To choose between different transformers for a given application, the TOC for each transformer is calculated and compared. The transformer with the lowest TOC represents the best choice from an economic standpoint.

Table 5.5. General Economic Assumptions

$\begin{array}{ll}\text { Economic life } & 30 \text { years } \\ \text { Depreciable life } & 20 \text { years } \\ \text { Property tax rate } & 2 \% / \text { year } \\ \text { Income tax rate } & 39.1 / \% \\ \text { Investment tax credit } & 0.0 \% \\ \text { Nominal discount rate } & 10.5 \% \\ \text { General inflation rate } & 6.0 \% / \text { year } \\ \text { Price year } & 1989 \\ \text { Construction period } & 1 \text { year }\end{array}$




\subsection{Results}

This section provides information on the key life-cycle cost results produced in the study. All results are shown in 1989 price levels, and all results in this section are based on the base case materials assumptions described earlier.

As mentioned previously, the life-cycle costs are determined for a wide range of alternative transformer designs for each technology. The design with the lowest life-cycle cost is then selected as the optimal design and provides the basis for comparison to other transformer technologies. This process is illustrated in Figure 5.2, which plots the life-cycle cost results of approximately 50 transformer designs. After the optimal design is identified, additional designs near the optimal are investigated as desired to fine tune the design selection. Also shown in Figure 5.2 is the contribution of annual energy losses to the total life-cycle cost. The results shown confirm that selecting transformers on the basis of efficiency will not provide a good choice from an economics perspective. The highly efficient transformers on the left side of the curve represent less economically attractive designs than the optimal point.

After the life-cycle cost model was completed for conventional transformers, a "reality check" was performed to determine if the purchase cost estimated for the transformers appeared to be within reasonable range of the purchase cost of transformers currently in use. The results of this comparison are shown in Figure 5.3. The solid boxes in Figure 5.3 represent the best purchase cost data obtained during the study to evaluate cost trends and develop the cost models; these data have been updated to 1989 price levels to be consistent with estimates from the EAST code. The three open boxes in Figure 5.3 show the purchase cost (including shipping) estimated by the EAST code for conventional transformers at 100,300, and 1000 MVA using "typical" transformer weight data provided by a member of the SAG. The fit of the EAST results to the data was judged to be very good for the purposes of this study.

The estimated life-cycle costs of grid transformers using laminated Si-steel cores are shown in Figure 5.4. Both of the HTS transformers show a significant cost advantage over the conventional transformer design at all power levels. The HTS cryostable (HTS-CS) transformer is slightly less expensive than the HTS ultrastable (HTS-US) transformer for all of the design points evaluated, although the difference is not likely significant within the overall design uncertainties present.

The differences in the transformer life-cycle costs can be best understood by breaking the overall life-cycle cost into three components: capital, energy losses, and O\&M. This cost breakdown is shown in Figures 5.5 through 5.7 for the 30-, 200-, and 1000-MVA grid transformers. For the conventional transformers, the value of energy losses represents the largest component of the life-cycle cost, followed closely by the transformer capital cost. In the case of the HTS-CS technology, the capital and energy loss contributions tend to be roughly equivalent. On the other hand, the HTS-US technology always has lower contributions from the energy loss component, although this is achieved at the expense of capital costs that are higher than for the HTS-CS case. The value of energy losses tends to make up a smaller fraction of the life-cycle cost at the smallest transformer size than for the larger sizes. 


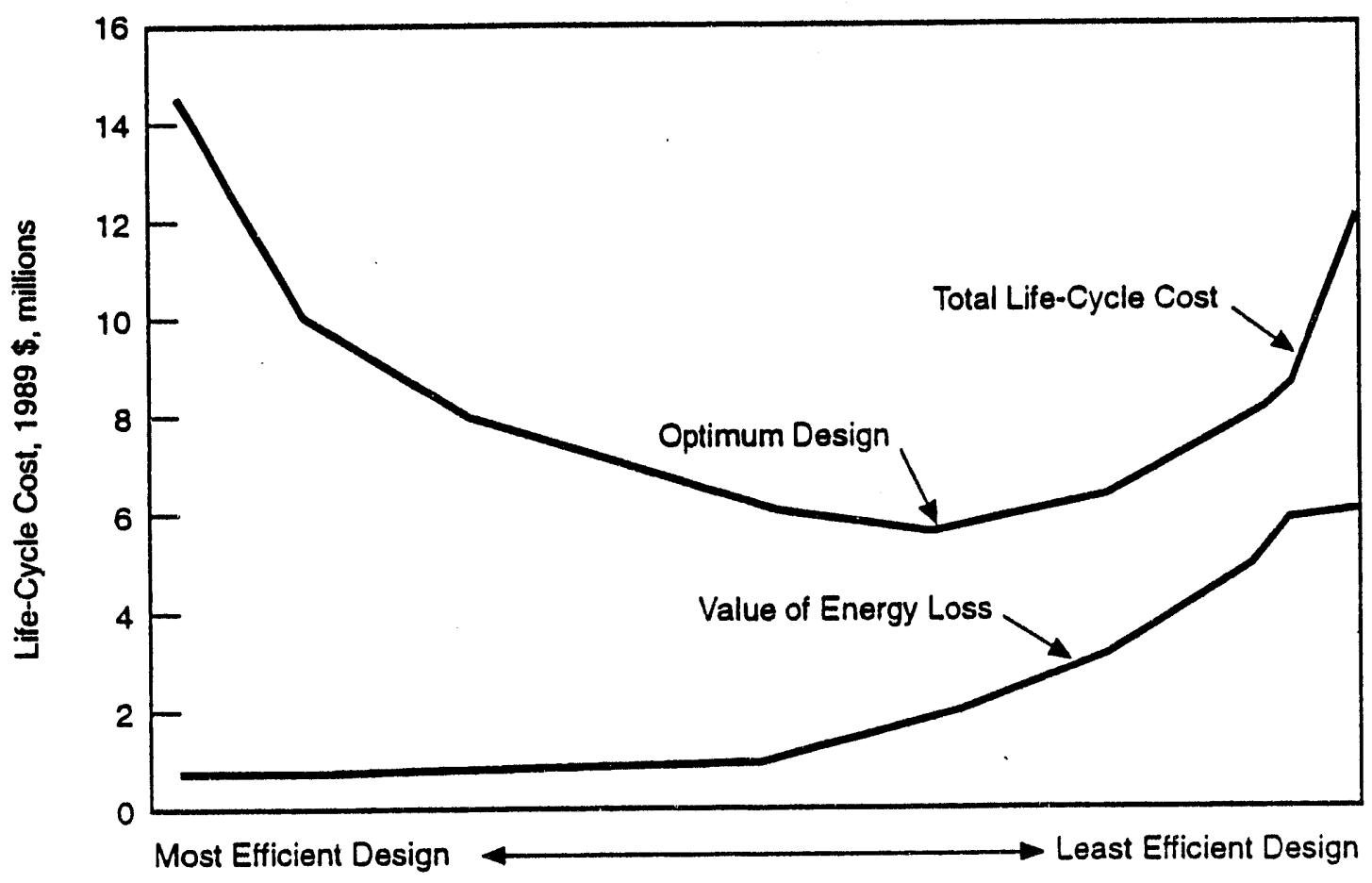

Figure 5.2. Example of Transformer Design Evaluation

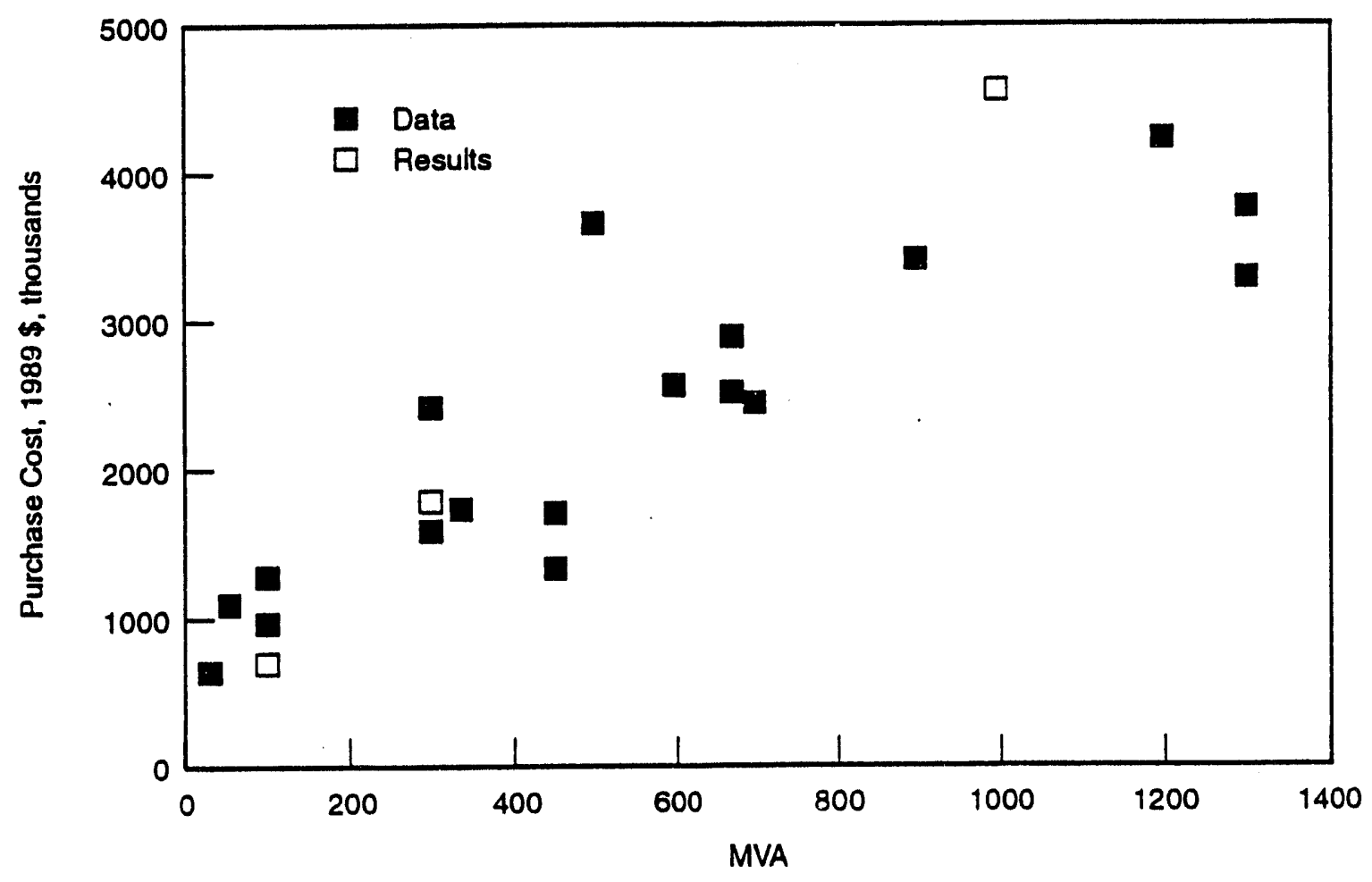

Figure 5.3. Comparison of EAST Model Test Results to Purchase Cost Data 


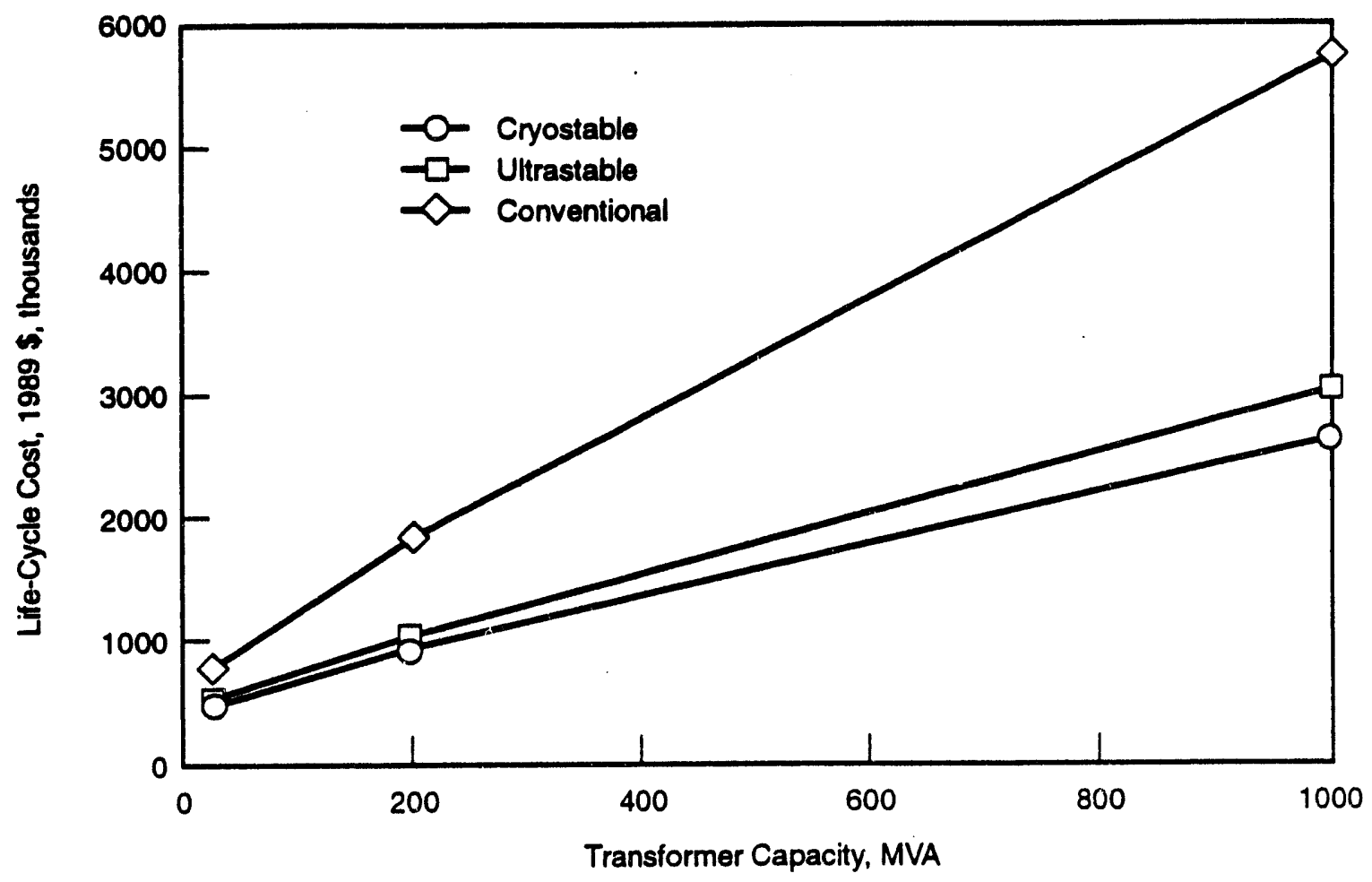

Figure 5.4. Life-Cycle Cost Comparison for Grid Transformers with Si-Steel Core

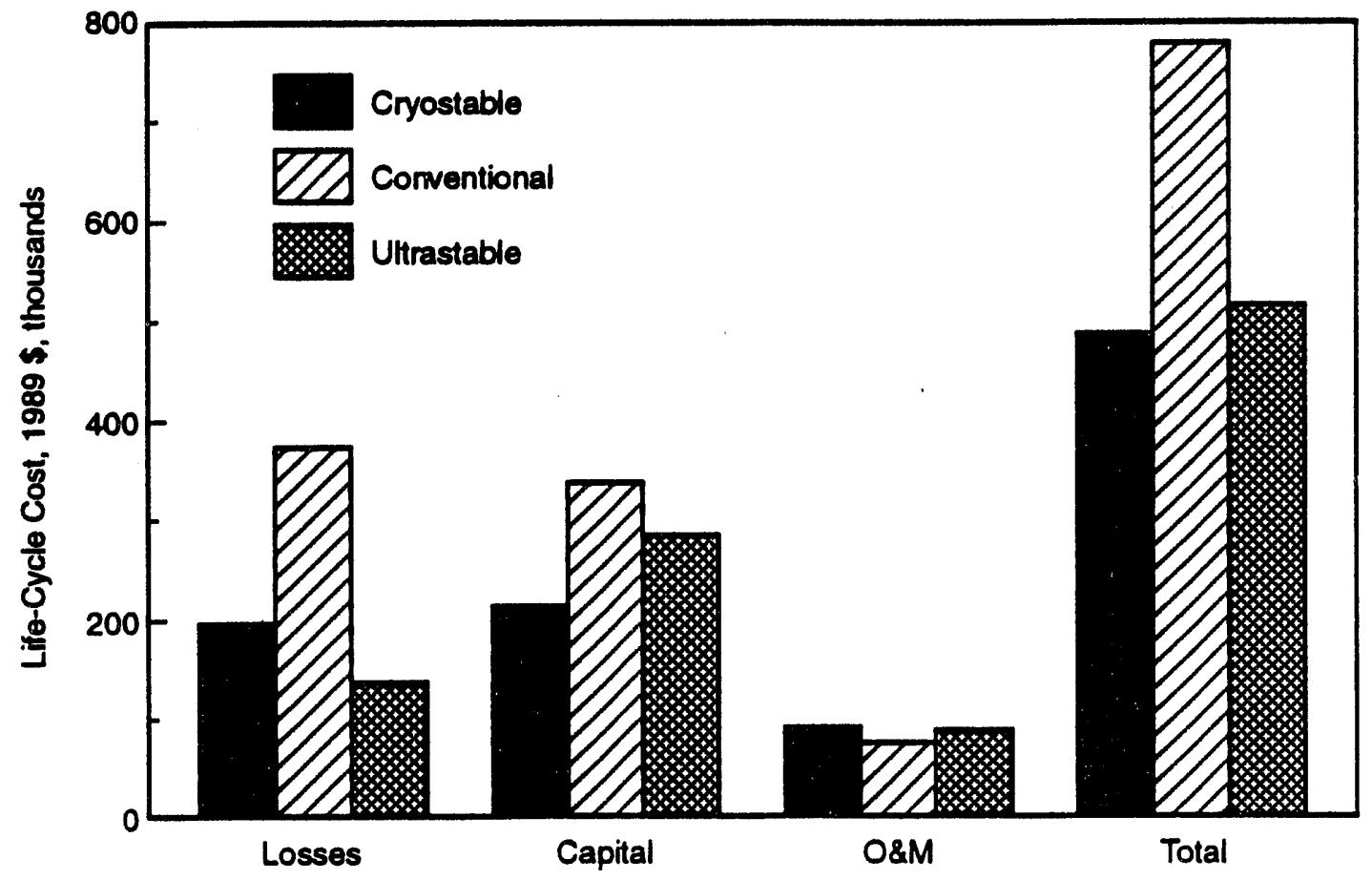

Figure 5.5. Life-Cycle Cost Breakdown for 30-MVA Grid Transformers with Si-Steel Core 


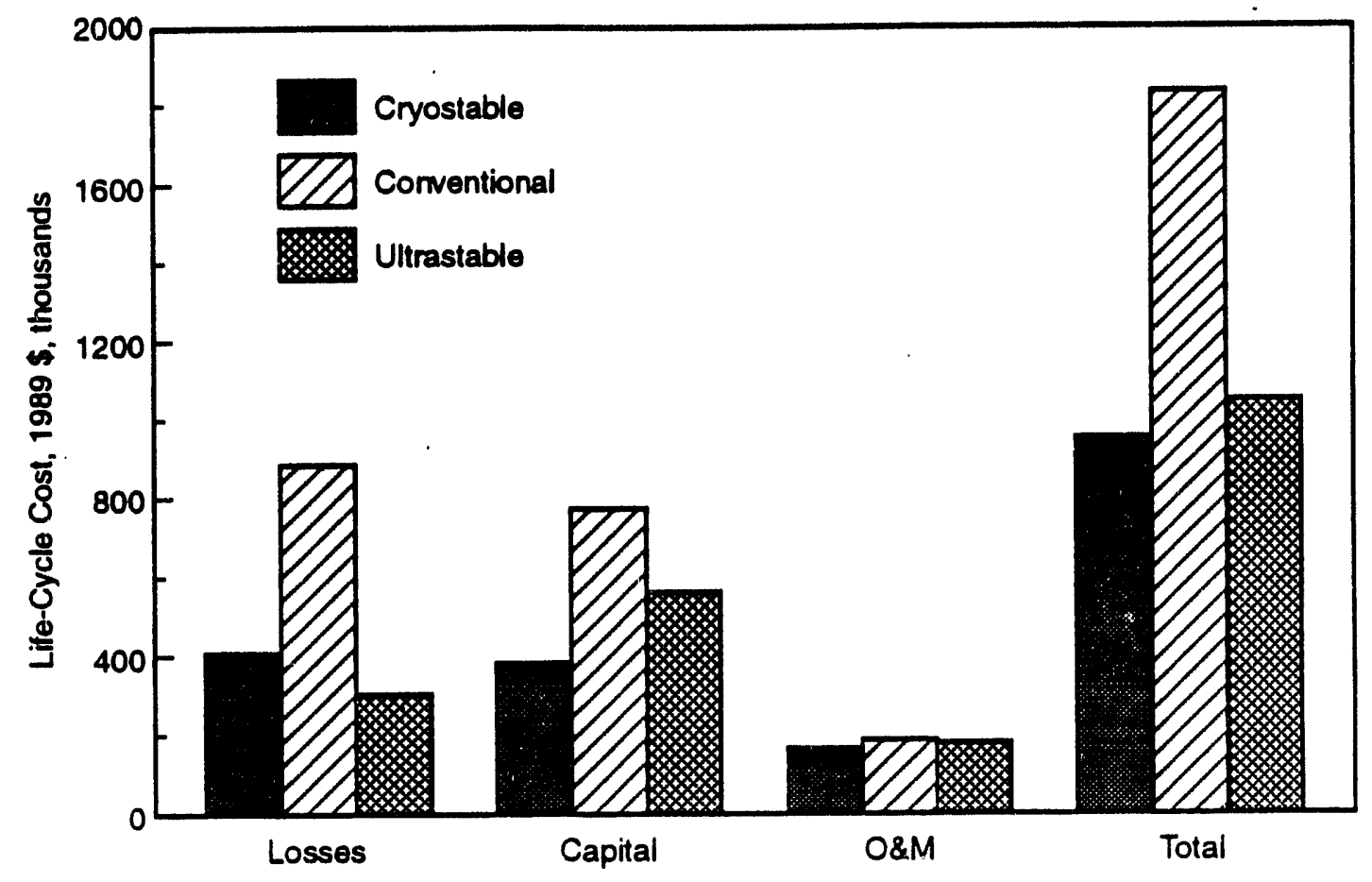

Figure 5.6. Life-Cycle Cost Breakdown for 200-MVA Grid Transformers with Si-Steel Core

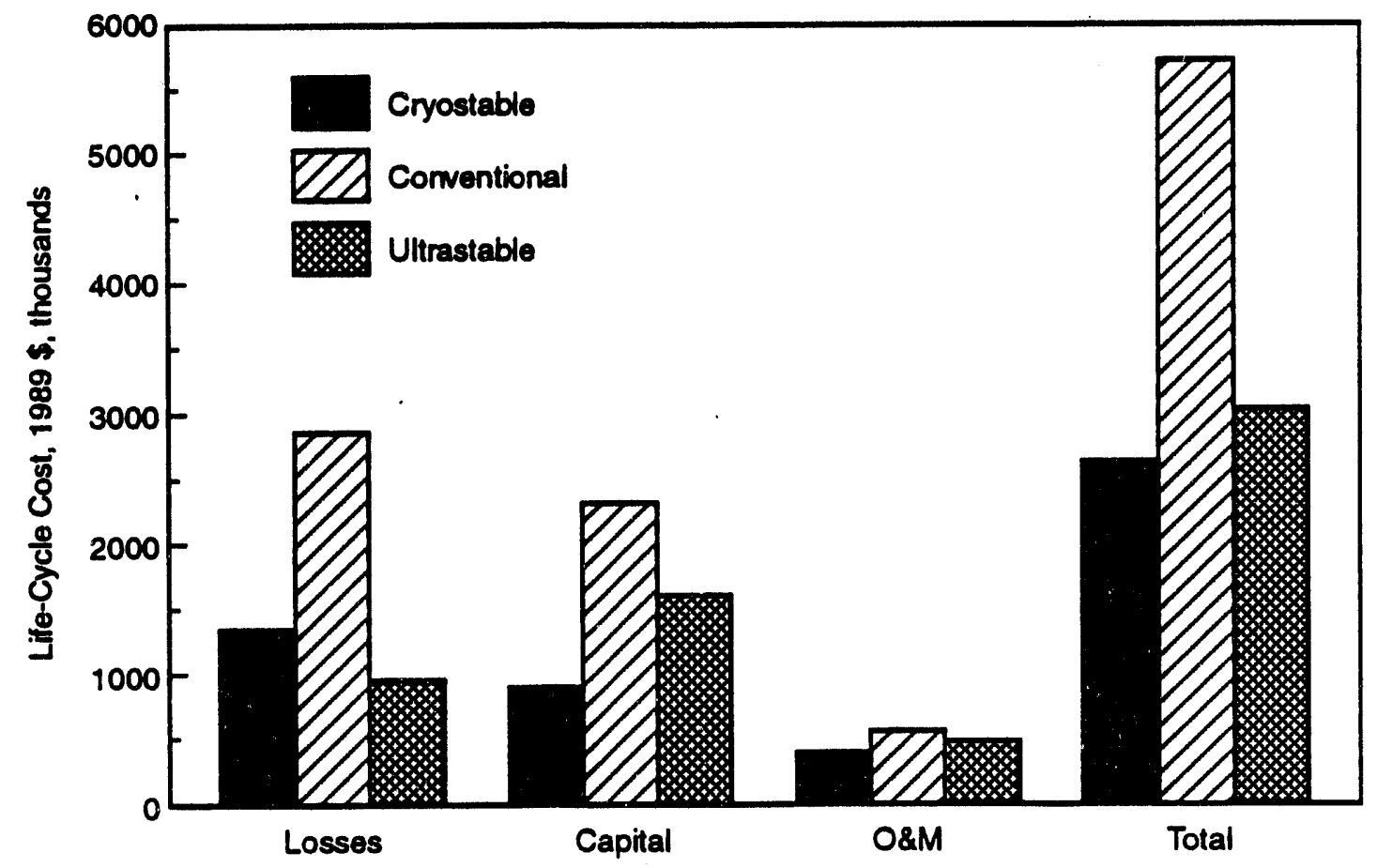

Figure 5.7. Life-Cycle Cost Breakdown for 1000-MVA Grid Transformers. with Si-Steel Core 
Several insights can be drawn from examining the life-cycle cost breakdowns for the grid transformers. First, the HTS transformers achieve a life-cycle cost advantage relative to the conventional technology both by being more efficient (lower losses) and by having a lower initial cost. Second, although the HTS-US technology design has much lower energy losses than the HTS-CS design, the added capital costs make it less desirable for the base case materials parameters. Finally, the O\&M costs for all the cases tend to make up a fairly small portion of the life-cycle cost and are roughly equivalent for each of the three technologies. Because annual O\&M expenses are estimated as a percentage of initial capital costs, the life-cycle cost contribution of O\&M tends to be a constant fraction of the capital cost contribution.

The estimated life-cycle costs for the step-up transformers using laminated Si-steel cores are show in in Figure 5.8. As with the grid transformers, the HTS designs show a large life-cycle cost advantage over the conventional transformer design at all power levels.

Because of how the transformers are operated, the breakdowns of the life-cycle costs for the step-up transformers, shown in Figures 5.9 through 5.11, are different from those for the grid transformers. While the grid transformers are assumed to be energized at all times and to operate at varying loads, the step-up transformers are assumed to operate only at full load and, thus, to have no energy losses when the power plant is off-line. The only exception to this is for the HTS

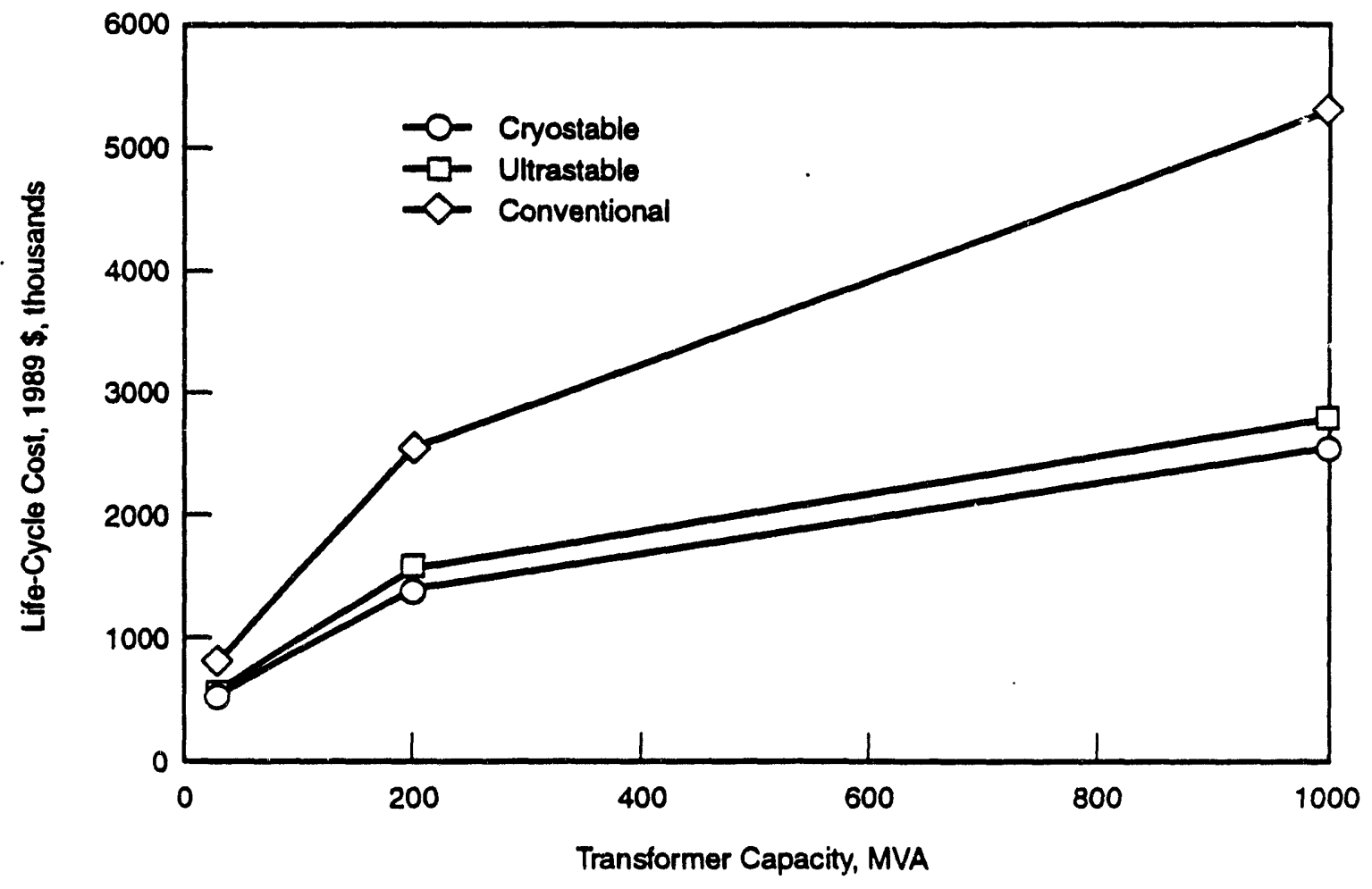

Figure 5.8. Life-Cycle Cost Comparison for Step-Up Transformers with Si-Steel Core 


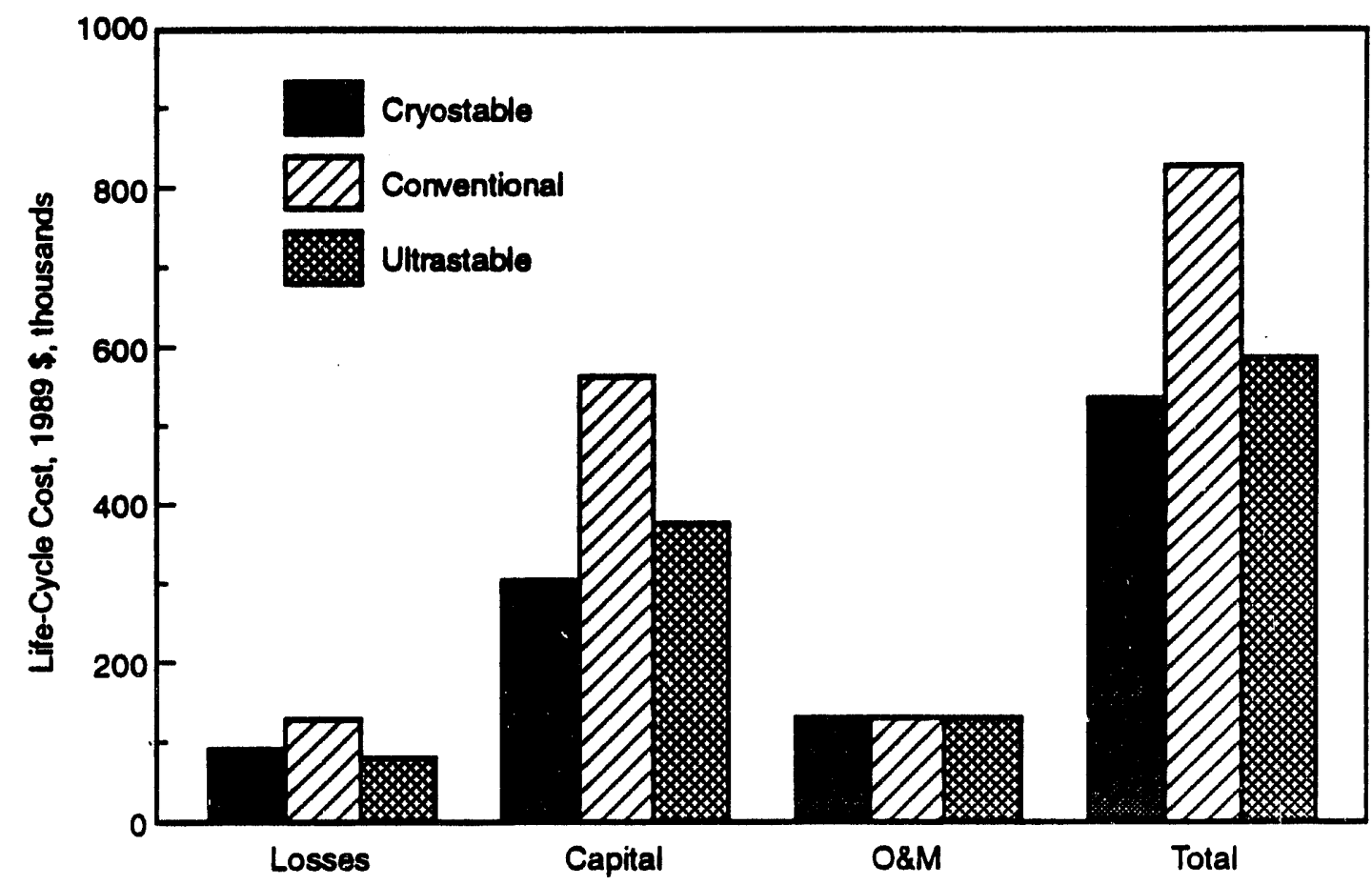

Figure 5.9. Life-Cycle Cost Breakdown for 60-MVA Step-Up Transformers with Si-Steel Core

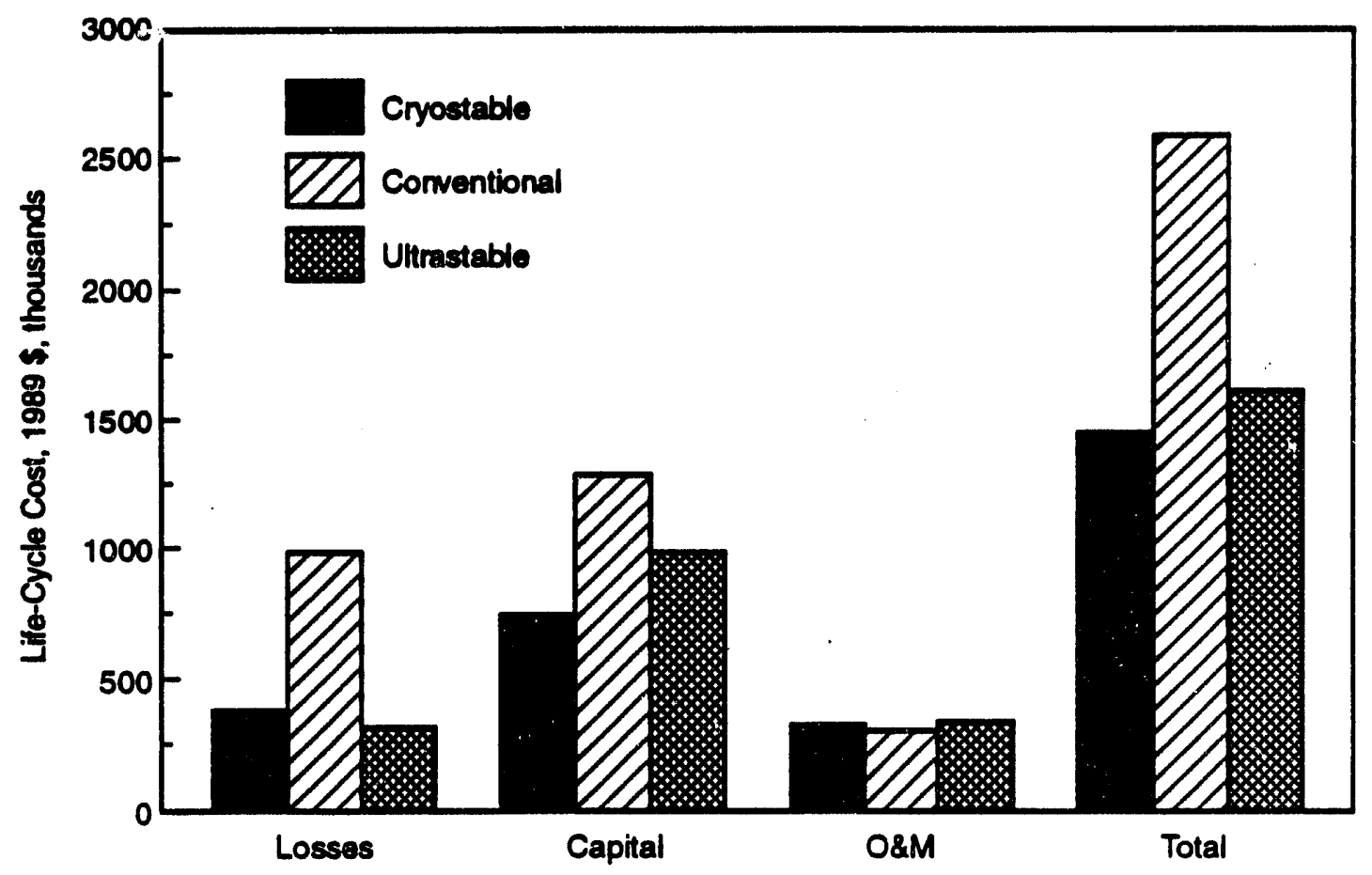

Figure 5.10. Life-Cycle Cost Breakdown for 310-MVA Step-Up Transformers with Si-Steel Core 


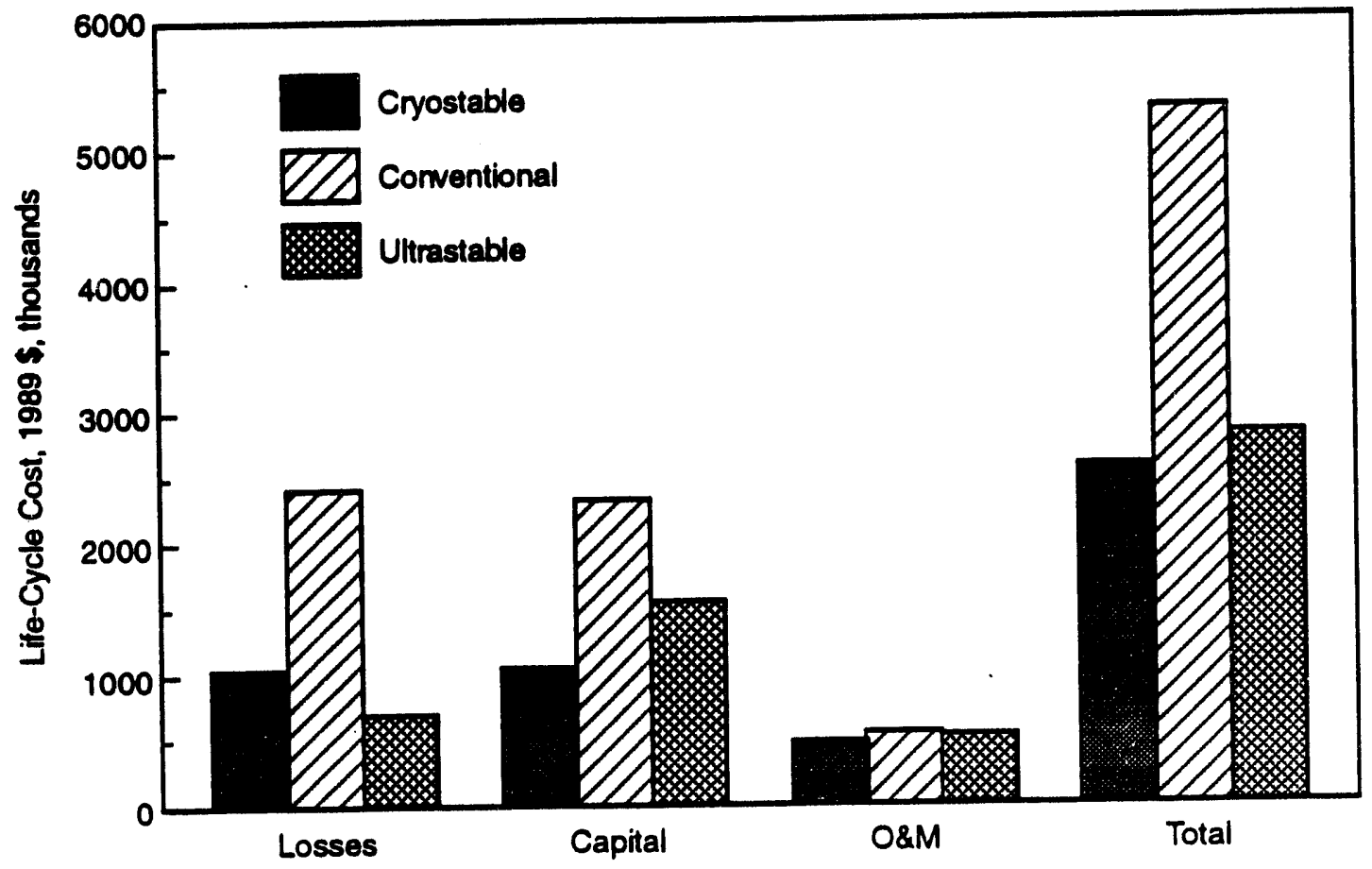

Figure 5.11. Life-Cycle Cost Breakdown for 630-MVA Step-Up Transformers with Si-Steel Core

transformers; here, it is assumed that the transformer would need to remain at operating temperature (to reduce start-up time and the effects of thermal cycling), so the cryocooler was assumed to operate throughout the year. The lower operating times for the step-up transformers and the absence of no-load losses combine to make the value of energy losses a smaller fraction of the life-cycle cost than they are for the grid transformers.

For the 60-MVA transformer case (Figure 5.9), the application is assumed to be a peaking plant with a capacity factor of $10 \%$. For this application, the value of energy losses makes up the smallest component of the life-cycle cost for all of the technologies. The HTS technologies achieve the bulk of their economic advantage relative to the conventional technology because of lower capital costs. In this application, the total life-cycle cost of the conventional transformer is roughly $50 \%$ greater than that of the HTS transformers.

The 310-MVA transformer application is for an intermediate load power plant with an annual capacity factor of $30 \%$. In this case, shown in Figure 5.10, the energy losses represent a significant fraction of the life-cycle cost for the conventional transformer. Energy losses are roughly on a par with O\&M costs for the HTS transformers, with capital costs dominating. In this application, with longer operating times and hence higher potential for energy savings than the peaking case, the conventional transformer has an overall life-cycle cost $70 \%$ to $80 \%$ more than that of the HTS transformers. 
A baseload power plant with an assumed annual capacity factor of $65 \%$ is the application for the 630-MVA transformer. The higher capacity factor provides the potential for the largest energy savings of any of the step-up transformers evaluated, and, in this case, the life-cycle cost of the conventional transformer is roughly double that of the HTS designs. A breakdown of the life-cycle costs is shown in Figure 5.11.

A complete breakdown of the transformer capital cost for the Si-steel core grid transformers is shown in Table 5.6, and for Si-steel core step-up transformers in Table 5.7. These capital costs are for the designs identified as optimal from a life-cycle cost standpoint. The capital cost estimates are shown to provide a general feeling of the relative importance of each capital cost element but should not be used to draw conclusions regarding the economic attractiveness of the technologies.

A summary of the annual O\&M cost estimates for the grid and step-up transformers is shown in Tables 5.8 and 5.9, respectively. As described earlier, the annual O\&M cost estimates were developed based on a percentage of the installed capital cost for the transformer and were broken into two components: the cryocooler and the transformer (i.e., all other maintenance activities).

Comparing the O\&M results among the three technologies shows them to be surprisingly similar. Although the cryocooler represents a significant activity that increases the cost of O\&M for the HTS transformers, the reduced capital cost of the HTS transformers decreases O\&M costs relative to the conventional transformer. Physical aspects of the HTS transformers that could reduce O\&M expenses relative to conventional transformers are the much smaller transformer size, significantly reduced volume of oil in the transformer core, and potential for advanced diagnostics/control systems, which may reduce maintenance.

The impact of amorphous transformer cores on the overall life-cycle cost of the transformers was found to be modest for all the technologies. These results are shown for the conventional and HTS-CS grid transformers in Figure 5.12. Although the amorphous core transformer had reduced load losses, it also had a higher initial cost that more than offset the reduction in energy losses. Because the amorphous cores tended to affect each of the technologies similarly, they did not change the relative comparisons among the technologies. The purpose of the analysis was to determine whether the amorphous core would affect the technology comparison, not to make definitive conclusions regarding the advantages of amorphous core transformers relative to Si-steel cores. More detailed evaluations would be required to draw specific conclusions about the economic benefits of amorphous cores in the transformer applications investigated. 
Table 5.6. Capital Cost Breakdown for Grid Transformers with Si-Steel Core

\begin{tabular}{|c|c|c|c|c|c|c|c|}
\hline & \multicolumn{7}{|c|}{ Cost. 1989 \$. Thousands } \\
\hline & Shell & Core & Windings & Shipping & $\begin{array}{c}\text { Cryogenic } \\
\text { Cooler } \\
\end{array}$ & Installation & Total \\
\hline \multicolumn{8}{|l|}{ Conventional } \\
\hline 30 MVA & 13 & 169 & 161 & 2 & 0 & 18 & 363 \\
\hline 200 MVA & 23 & 331 & 351 & 100 & 0 & 24 & 829 \\
\hline 1000 MVA & 70 & 970 & 1208 & 200 & 0 & 36 & 2485 \\
\hline \multicolumn{8}{|l|}{ HTS-US } \\
\hline 30 MVA & 5 & 72 & 131 & 2 & 88 & 12 & 311 \\
\hline 200 MVA & 10 & 141 & 277 & 2 & 153 & 16 & 598 \\
\hline 1000 MVA & 28 & 398 & 846 & 100 & 339 & 24 & 1734 \\
\hline \multicolumn{8}{|l|}{ HTS-CS } \\
\hline 30 MVA & 4 & 57 & 20 & 2 & 132 & 11 & 227 \\
\hline 200 MVA & 8 & 116 & 45 & 2 & 227 & 15 & 412 \\
\hline 1000 MVA & 17 & 228 & 165 & 2 & 551 & 19 & 982 \\
\hline
\end{tabular}

Table 5.7. Capital Cost Breakdown for Step-Up Transformers with Si-Steel Core

\begin{tabular}{|c|c|c|c|c|c|c|c|}
\hline & \multicolumn{7}{|c|}{ Cost. 1989 \$. Thousands } \\
\hline & Shell & Cole & Windings & Shipping & $\begin{array}{c}\text { Cryogenic } \\
\text { Cooler } \\
\end{array}$ & Installation & Total \\
\hline \multicolumn{8}{|l|}{ Conventional } \\
\hline 6 MVA & 19 & 247 & 223 & 100 & 0 & 21 & 609 \\
\hline 310 MVA & 46 & 594 & 518 & 200 & 0 & 29 & 1386 \\
\hline 630 MVA & 79 & 1043 & 1160 & 200 & 0 & 37 & 2518 \\
\hline \multicolumn{8}{|l|}{ HTS-US } \\
\hline 6 MVA & 8 & 98 & 148 & 2 & 136 & 14 & 406 \\
\hline 310 MVA & 20 & 221 & 480 & 2 & 310 & 19 & 1052 \\
\hline $630 \mathrm{MVA}$ & 32 & 399 & 711 & 100 & 427 & 24 & 1693 \\
\hline \multicolumn{8}{|l|}{ HTS-CS } \\
\hline 6 MVA & 6 & 77 & 26 & 2 & 206 & 13 & 329 \\
\hline 310 MVA & 19 & 224 & 84 & 2 & 456 & 19 & 804 \\
\hline 630 MVA & 19 & 221 & 168 & 2 & 696 & 19 & 1125 \\
\hline
\end{tabular}


Table 5.8. Annual O\&M Cost Results for Grid Transformers

Cost. 1989 \$. Thousands

\begin{tabular}{ccc} 
Transformer & Cryocooler & Total \\
\cline { 2 - 3 } 7 & 0 & 7 \\
17 & 0 & 17 \\
50 & 0 & 50
\end{tabular}

HTS-US

30 MVA

200 MVA

1000 MVA

4

49

$\begin{array}{lll}9 & 8 & 17\end{array}$

$\begin{array}{lll}28 & 17 & 45\end{array}$

HTS-CS

30 MVA

200 MVA

1000 MVA

2

$7 \quad 9$

$11 \quad 15$

$\begin{array}{lll}4 & 11 & 15 \\ 9 & 28 & 36\end{array}$

Note: numbers may not add because of rounding.

Table 5.9. Annual O\&M Cost Results for Step-Up Transformers

Cost. 1989 \$. Thousands

\begin{tabular}{cccc} 
& Transformer & Cryocooler & Total \\
\cline { 2 - 3 } Conventional & & & \\
60 MVA & 12 & 0 & 12 \\
310 MVA & 28 & 0 & 28 \\
630 MVA & 50 & 0 & 50
\end{tabular}

HTS-US

60 MVA

310 MVA

630 MVA

5

15

$7 \quad 12$

$16 \quad 30$

25

21

47

HTS-CS

60 MVA

310 MVA

630 MVA

$\begin{array}{lll}2 & 10 & 13 \\ 7 & 23 & 30 \\ 9 & 35 & 43\end{array}$

Note: numbers may not add because of rounding. 


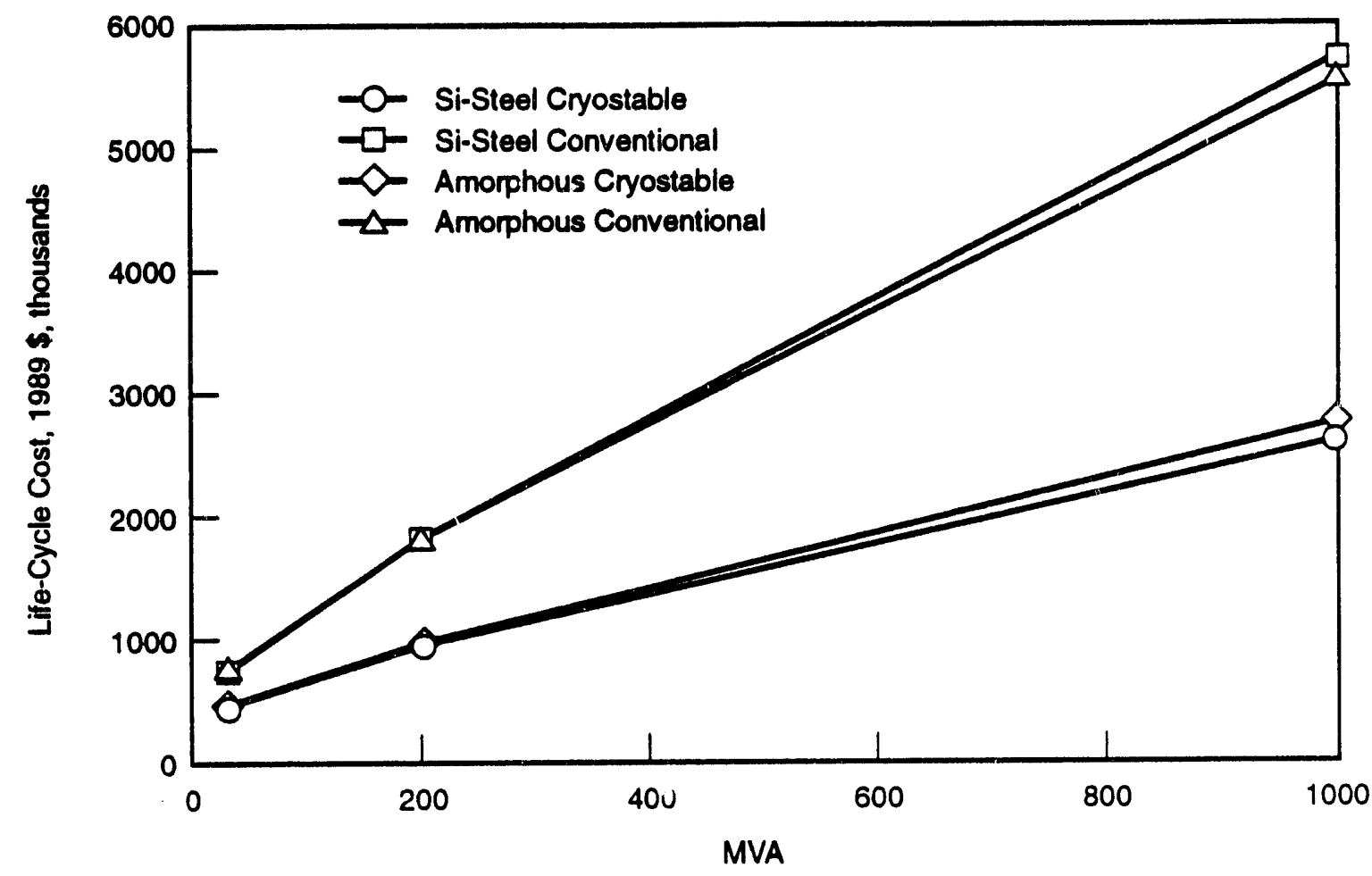

Figure 5.12. Comparison of Amorphous and Si-Steel Core Life-Cycle Costs for HTS-CS and Conventional Transformers

\subsection{Conclusions of Life-Cycle Cost Analysis}

A large amount of information was generated in the evaluation of the alternative transformer technologies. The key conclusions related to the life-cycle cost that appear to be justified for the base case materials parameters are as follows:

- Both the HTS-CS and the HTS-US technologies appear to show significant life-cycle cost advantages relative to conventional transformers. Compared with the conventional transformers, the HTS technologies appear to have the potential for both lower energy losses and lower capital costs.

- For the base case materials assumptions, the HTS-CS has a slight life-cycle cost advantage (4\% to $14 \%$ ) over the HTS-US technology. Within the overall uncertainty of this study, neither of these approaches to designing HTS transformers appears to have a clear economic superiority for the base case materials assumptions.

- The use of amorphous cores in transformer designs does not appear to impact the economic attractiveness of HTS transformers relative to conventional transformers. 


\subsection{Sensitivity Analyses}

This chapter describes the key sensitivity analyses performed in the study. We focused on two areas that are very important in the design of HTS transformers: the critical current density of the HTS materials and the effects of self-fields. Our results indicate that the effect of increasing the critical current from low values to higher values does not always represent an improvement in the transformer performance. In actuality, an optimal critical current (from a life-cycle cost standpoint) will exist for transformers because some losses, such as hysteresis and eddy currents, are a function of $J_{c}$, increasing as $J_{c}$ is increased. The examination of the self-field impacts indicates that this is a critical performance parameter that can dramatically impact the losses, efficiency, and costs of HTS transformers.

\subsection{Influence of Critical Current Density on Transformer Performance, Design, and Costs}

Critical current density $\left(\mathrm{J}_{\mathrm{c}}\right)$ has a major influence on many aspects of superconductive transformer design. The value of $\mathrm{J}_{\mathrm{c}}$ at a given temperature and magnetic field intensity affects most major power losses in the transformer. The number of turns in the transformer windings, the size and weight of the transformer core, the transformer operating efficiency, and the overall transformer capital and operating costs are among those factors directly affected by the value used for $J_{c}$.

In a transformer of a given terminal voltage and power rating, a large number of design and performance parameters interact in a relatively complex manner, producing myriad designs from which the optimum design can be selected (i.e., the design that minimizes the transformer life-cycle cost). To determine the impact of $J_{c}$ on the optimum transformer design, a sensitivity analysis was performed. The results are discussed in this section.

\subsubsection{Sensitivity Analysis with $J_{c}$ for Cryostable Transformer Performance and Design}

Superconductive materials suitable for HTS transformer windings are not available yet. However, as the technology advances, it may be possible to fabricate suitable conductors having $\mathrm{J}_{\mathrm{c}}$ in excess of $10^{6} \mathrm{~A} / \mathrm{cm}^{2}$ at $77 \mathrm{~K}$ and in the magnetic fields anticipated in HTS transformers. Anticipating significant advances in HTS materials technology, our sensitivity analysis encompasses the range of critical current density from $10^{3}$ to $10^{7} \mathrm{~A} / \mathrm{cm}^{2}$.

Figure 6.1 shows the effect on the major loss mechanisms of varying $\mathrm{J}_{\mathrm{c}}$ from $10^{3}$ to $10^{7} \mathrm{~A} / \mathrm{cm}^{2}$ in cryostable superconductive transformer optimum designs. All of the transformers have the same design specifications (1000-MVA, 500/230-kV, $3 \phi$ autotransformer), with corresponding variations in the superconductor critical current density. All designs presented represent the minimum life-cycle cost.

The extreme variability of the various transformer losses with changing values of $J_{c}$ is shown in Figure 6.1. The plot reveals that the dominant loss through $10^{4} \mathrm{~A} / \mathrm{cm}^{2}$ is transformer core iron loss. The eddy-current loss in the cryostable superconductor windings increases with $J_{c}$ and dominates the 


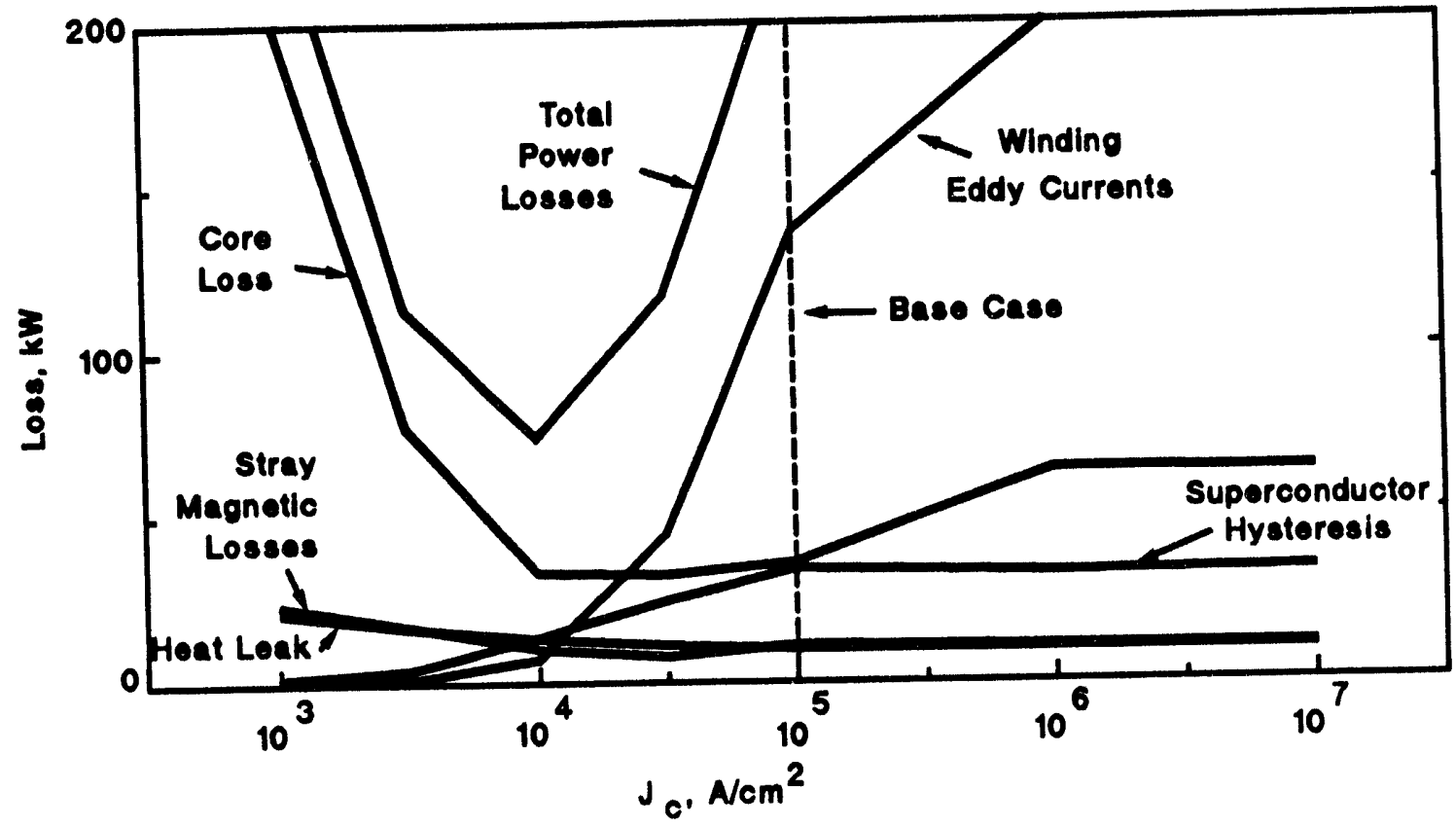

Figure 6.1. Cryostable Transformer Individual Power Losses Versus Critical Current Density $\left(\mathrm{J}_{\mathrm{c}}\right)$ (1000-MVA Autotransformers)

transformer losses at critical current densities beyond about $10^{4.5} \mathrm{~A} / \mathrm{cm}^{2}$. These losses and the other major loss components of the superconductive transformer will be discussed individually for the cryostable transformer designs as a function of $\mathrm{J}_{\mathrm{c}}$.

Many of the loss components in the superconducting transformer are strongly influenced by changes in various design aspects of the transformer as the critical current density is varied.

Table 6.1 contains a summary of some of the important design results affected by changes in $\mathrm{J}_{\mathrm{c}}$ for optimized 1000-MVA cryostable autotransformer designs. These will be discussed in the following sections. To better understand the processes involved, the critical current densities are divided into three $\mathrm{J}_{\mathrm{c}}$ ranges: low, intermediate, and high.

\subsubsection{Cryostable Transformer Performance and Design at Low $\mathrm{J}_{\mathrm{c}}$}

The transformer design optimization process minimizes the life-cycle cost associated with eaih design. For low critical current densities, the optimization process tends to offset the relatively large superconductive material requirements by increasing the core cross-sectional area, which, in turn, reduces the number of turns required.

For a constant core magnetic flux density, the core losses per kilogram of material are fixed. Because magnetic flux density within the core of the optimized transformer designs does not vary significantly as a function of $\mathrm{J}_{\mathrm{c}}$, the core losses correspond primarily to the amount of transformer core material. By increasing the amount of material in the transformer core, relatively fewer turns 
Table 6.1. Key Design Results for 1000-MVA Cryostable Autotransformers as a Function of $J_{c}$

\begin{tabular}{|c|c|c|c|c|c|c|c|}
\hline \multirow[b]{2}{*}{$\underline{\log , J_{c}}$} & \multicolumn{3}{|c|}{ Material Mass, kg } & \multicolumn{2}{|c|}{$\begin{array}{l}\text { Average Winding } \\
\text { Fields }\end{array}$} & \multicolumn{2}{|c|}{ Conductor Radii } \\
\hline & Core & $\mathrm{Cu}$ & $\mathrm{SC}$ & $\underline{B}_{L 1}, G$ & $\underline{B}_{L 2}, G$ & $\mathbf{R}_{\text {wirel }} \mathrm{mm}$ & $\underline{\mathbf{R}}_{\text {wire2 }} \mathrm{mm}$ \\
\hline 3 & 183,300 & 61.91 & 10,630 & 6.044 & 6.044 & 10.22 & 11.05 \\
\hline 3.5 & 58,080 & 164.8 & 5,061 & 18.27 & 18.25 & 5.780 & 6.251 \\
\hline 4 & 22,240 & 409.5 & 2,305 & $52-96$ & 52.68 & 3.347 & 3.628 \\
\hline 4.5 & 24,050 & 552.3 & 627.6 & 128.9 & 126.5 & 2.127 & 2.321 \\
\hline 5 & 24,360 & 655.6 & 181.1 & 220.0 & 212.1 & 1.627 & 1.791 \\
\hline 6 & 52,660 & 561.5 & 13.42 & 295.1 & 280.7 & 1.402 & 1.554 \\
\hline 7 & 52,560 & 570.3 & 1.342 & 304.8 & 289.5 & 1.380 & 1.530 \\
\hline
\end{tabular}

are required in the superconductive transformer windings. An increase in the core volume does result in increased core losses; however, the winding volume with its associated relatively high material cost is reduced.

\subsubsection{Cryostable Transformer Performance and Design at Intermediate $\mathbf{J}_{c}$}

In Figure 6.1, the core losses (and core volume) are shown to reach a minimum between $10^{4}$ and $10^{5} \mathrm{~A} / \mathrm{cm}^{2}$. As the critical current density is increased, the amount of superconductive material required for a given number of turns is correspondingly reduced. The transformer life-cycle cost components associated with the core material and core iron losses then begin to become relatively more significant. Thus, the optimum transformer design at the intermediate values of $\mathrm{J}_{\mathrm{c}}$ has a higher number of turns in the windings and a smaller core volume. Hence, the transformer core losses at intermediate values of $\mathrm{J}_{\mathrm{c}}$ are less than in a comparable, low $\mathrm{J}_{\mathrm{c}}$ design.

However, as $J_{c}$ is increased, the wire radii decrease, so the self-field component of the average winding field increases (see Section 3.4.2). This in turn, increases both the eddy-current losses (see Section 3.4.5), which vary with the square of the average winding field, and the hysteresis losses (see Section 3.4.4), which vary linearly with the average winding field. At a $J_{c}$ of $10^{4} \mathrm{~A} / \mathrm{cm}^{2}$ and above, a relatively large amount of copper is required to maintain cryostability within the conductor. The eddy-current loss becomes the dominant loss component for values of $J_{c}$ above $10^{4} \mathrm{~A} / \mathrm{cm}^{2}$.

The overall effect of these tradeoffs is that the maximum transformer efficiencies and minimum life-cycle costs are attained in the intermediate range of the critical current density (see Figures 6.2 and 6.3).

\subsubsection{Cryostatle "ransformer Performance and Design at High $\mathbf{J}_{\mathbf{c}}$}

At high values of $J_{c}\left(10^{5} \mathrm{~A} / \mathrm{cm}^{2}\right.$ and above) the designs are completely dominated by the eddycurrent losses in the stabilizer. Two factors influence eddy-current losses in the windings: 


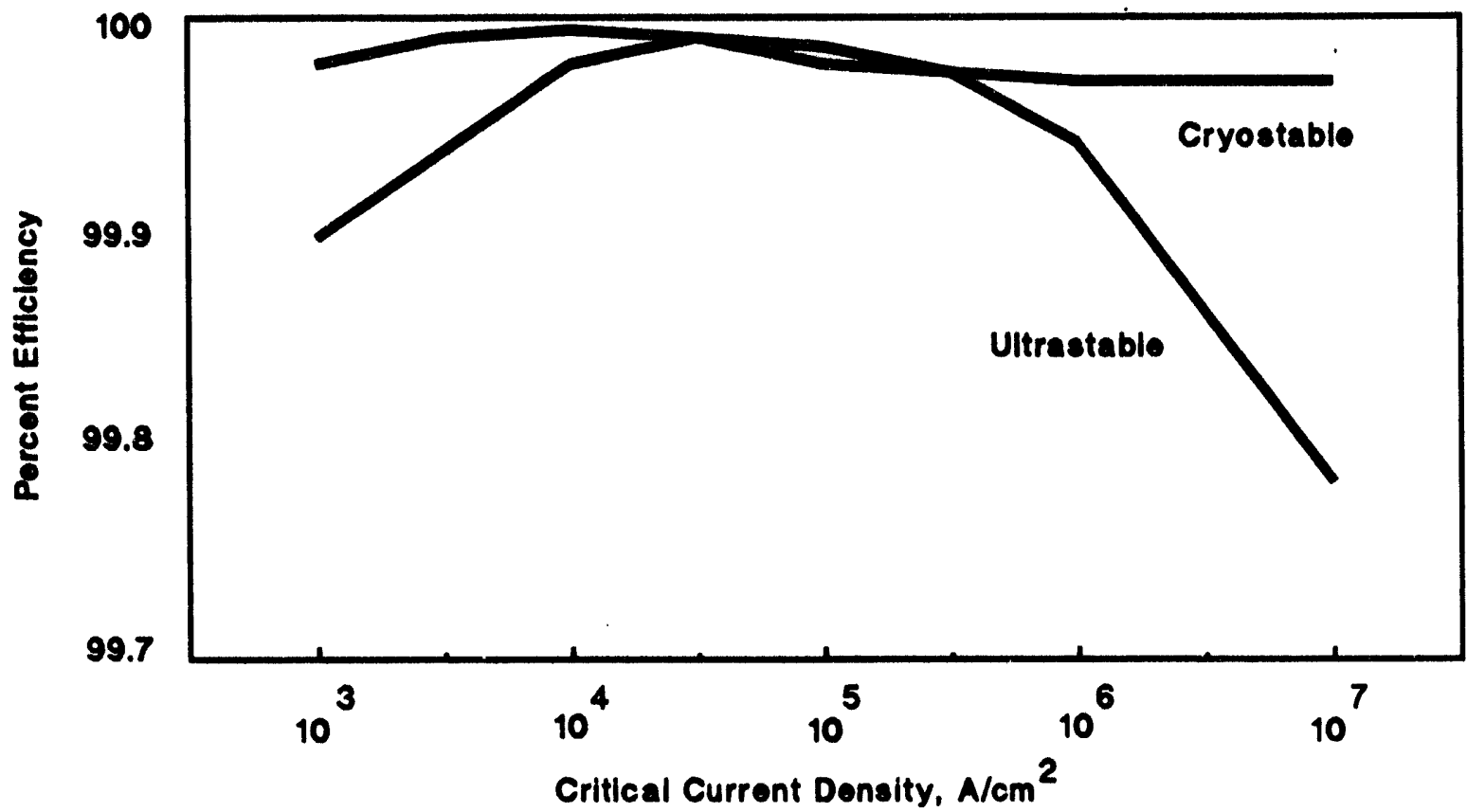

Figure 6.2. Transformer Efficiency as a Function of $\mathrm{J}_{\mathrm{c}}$ (1000-MVA Autotransformers)

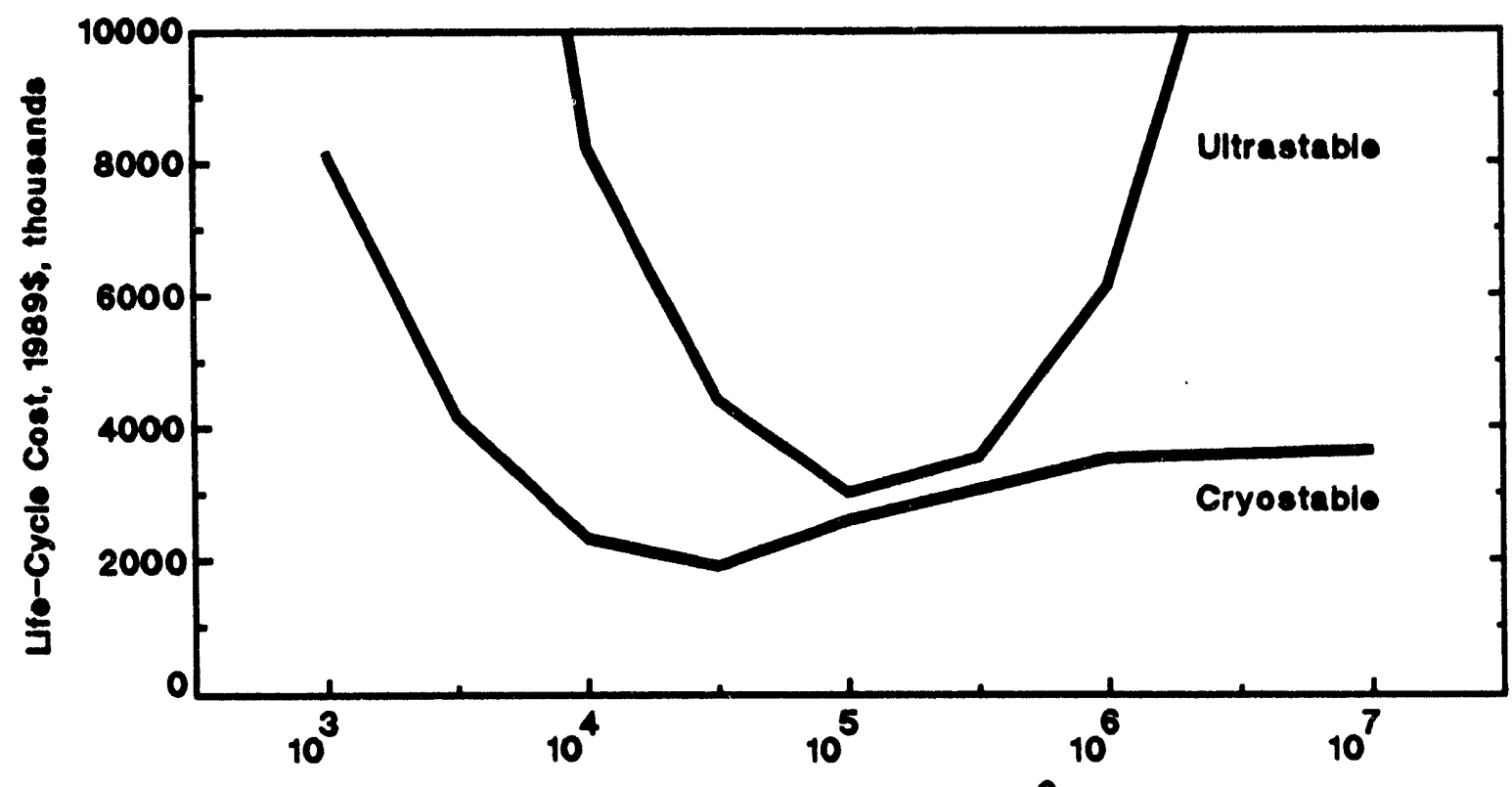

Critical Current Donsity, $\mathrm{Acm}^{2}$

Figure 6.3. Transformer Life-Cycle Cost as a Function of $J_{c}$ (1000-MVA Autotransformers) 
- copper volume - Increased amounts of copper increase the eddy-current losses.

- average winding field - The magnetic field intensity at the conductor is a key factor in eddycurrent losses.

All of the other terms in the eddy-current loss equation are constant as $\mathrm{J}_{c}$ is varied over the range of analysis.

The copper volume within the cryostable transformer varies as the current density increases. One of the principal design parameters requires that proportionately more copper is required in the conductor to maintain cryostability as $\mathrm{J}_{\mathrm{c}}$ is increased because of the surface area heat transfer requirement (see Section 3.4.3).

Another important design parameter, which determines the magnitude of the eddy-current loss, is the volume of the copper in the transformer windings. The copper volume depends on the length of the conductors, their cross-sectional areas, and the ratio of copper to superconductor in the winding. The conductor length is, in turn, determined by the number of turns in the transformer winding and cross-sectional area of the core. The cross-sectional areas of the winding conductors are determined by the rated current, the resistivity of the conductor, $J_{c}$, and the operating margin. These factors change as part of the optimization process. For example, as the cost associated with increased eddy-current losses increases, the copper volume is decreased by reducing the number of turns in the windings. Thus, in the optimum transformer design, the increases in winding losses for higher $J_{c}$ designs are compensated for by the addition of more core material, with corresponding increases in the core weight and losses.

The average winding magnetic field also varies with $\mathrm{J}_{\mathrm{c}}$ and other design parameters. The self-field magnitude varies with strand radius (which is dependent on $\mathrm{J}_{\mathfrak{c}}$, the fraction of superconductor in the conductor, and the current operating margin), and the load current. The leakage field (from the core) is dependent on the core flux density and, hence, is a function of the overall optimization process.

Thus, as the eddy-current losses start to dominate the transformer designs at the higher values of $J_{c}$, the transformer design optimization routine begins to reduce the number of turns in the transformer windings and to increase the volume of the transformer core.

\subsubsection{Life-Cycle Cost Impacts for the Cryostable Transformer Designs}

Changes in the value of $J_{c}$ affect the life-cycle cost by changing the transformer materials requirements and impacting the transformer energy losses. The first-order changes in materials requirements occur in the volume of the HTS materials and the cryostability material, which are directly impacted by the level of $\mathrm{J}_{\mathrm{c}}$. Other components of the transformer are also affected. As the size of the windings changes, the transformer core, shell, and cryocooler must be resized. The materials changes also affect the transformer O\&M cost, which is estimated to be proportional to the transformer capital cost. 
The impact on life-cycle cost was determined by running the design and cost models for various values of $J_{c}$ and allowing the codes to develop an optimal transformer design. The results of the analysis indicate the lowest life-cycle cost that could be achieved at each of the $J_{c}$ values. The impact of various $\mathrm{J}_{\mathrm{c}}$ levels on the transformer life-cycle cost (for optimized designs) is shown in Figure 6.4. The results indicate that there is an optimal $J_{c}$ for both types of transformers and that the impact of $J_{c}$ is very different for the two design approaches. For the ultrastable design, the impact of $J_{c}$ is profound, with a sharp optimal region. The results suggest that the minimum $J_{c}$ required for an ultrastable design to build transformers that are economically superior to conventional transformers is somewhere above $10^{4} \mathrm{~A} / \mathrm{cm}^{2}$. In comparison, the impact of $\mathrm{J}_{\mathrm{c}}$ on the life-cycle cost of the cryostable design is less pronounced; the results indicate that a cryostable transformer could be economically superior to a conventional transformer with a minimum $J_{c}$ above $10^{3} \mathrm{~A} / \mathrm{cm}^{2}$.

The life-cycle cost impacts of $J_{c}$ can be best understood by breaking out the impacts of $J_{c}$ on the capital cost and energy losses of the transformer (O\&M expenses, because they are proportional to capital cost, follow capital cost trends). These results are shown in Figure 6.5 for the cryostable design. At a $\mathrm{J}_{\mathrm{c}}$ of $10^{3}$, the capital costs are high because of the large materials requirements, and the transformer core losses result in a large value of energy losses. As $\mathrm{J}_{\mathrm{c}}$ is increased, the transformer size and energy losses both decrease rapidly until a value of $J_{c}$ around $10^{4}$. At higher values of $J_{c}$, the eddy-current losses begin to rise rapidly. The result is an increase in the energy losses and the transformer capital costs as the optimal transformer design changes in an attempt to minimize the impacts of increasing eddy-current losses.

The capital and energy costs are high at low values of $\mathrm{J}_{\mathrm{c}}$ and decline as $\mathrm{J}_{\mathrm{c}}$ approaches a point around $10^{4}$. At higher values of $\mathrm{J}_{\mathrm{c}}$, the stabilizer requirement increases so the eddy-current losses in the winding stability material increase. This drives up the value of lost energy and (through the optimization process) the transformer capital costs.

\subsubsection{Summary of $\mathrm{J}_{\mathrm{c}}$ Sensitivity Analysis for Cryostable Designs}

At low values of $\mathrm{J}_{\mathrm{c}}$ (up to $10^{4} \mathrm{~A} / \mathrm{cm}^{2}$ ), the winding conductors are relatively large. To minimize the high material cost in the superconducting windings, the core is made large to minimize the number of winding turns required to support the terminal voltage of the transformer. The larger core volume yields higher total core losses and somewhat higher core material cost.

As $\mathrm{J}_{\mathrm{c}}$ increases to the intermediate range $\left(10^{4}\right.$ to $\left.10^{5} \mathrm{~A} / \mathrm{cm}^{2}\right)$, the winding cross-sectional areas are reduced, which reduces the amount of material required for the windings. More turns are assigned to the optimal transformer winding to reduce the core material requirements. This reduction in the conductor radii is accompanied by an increase in the magnetic self-field intensity to which the windings are subjected. This increase in magnetic flux leads to higher eddy-current losses, higher superconductor hysteresis losses, and an increase in total power losses.

As the $J_{c}$ of the superconductor is increased still further to the high range $\left(10^{5} \mathrm{~A} / \mathrm{cm}^{2}\right.$ and above), the increase in the eddy-current losses (due to the increased copper requirements per unit length of conductor and the larger average winding fields) becomes the most significant factor in the life-cycle cost of the optimized transformer designs. These losses are again compensated for by reducing the number of winding turns, leading to a heavier core with higher losses. The result of 


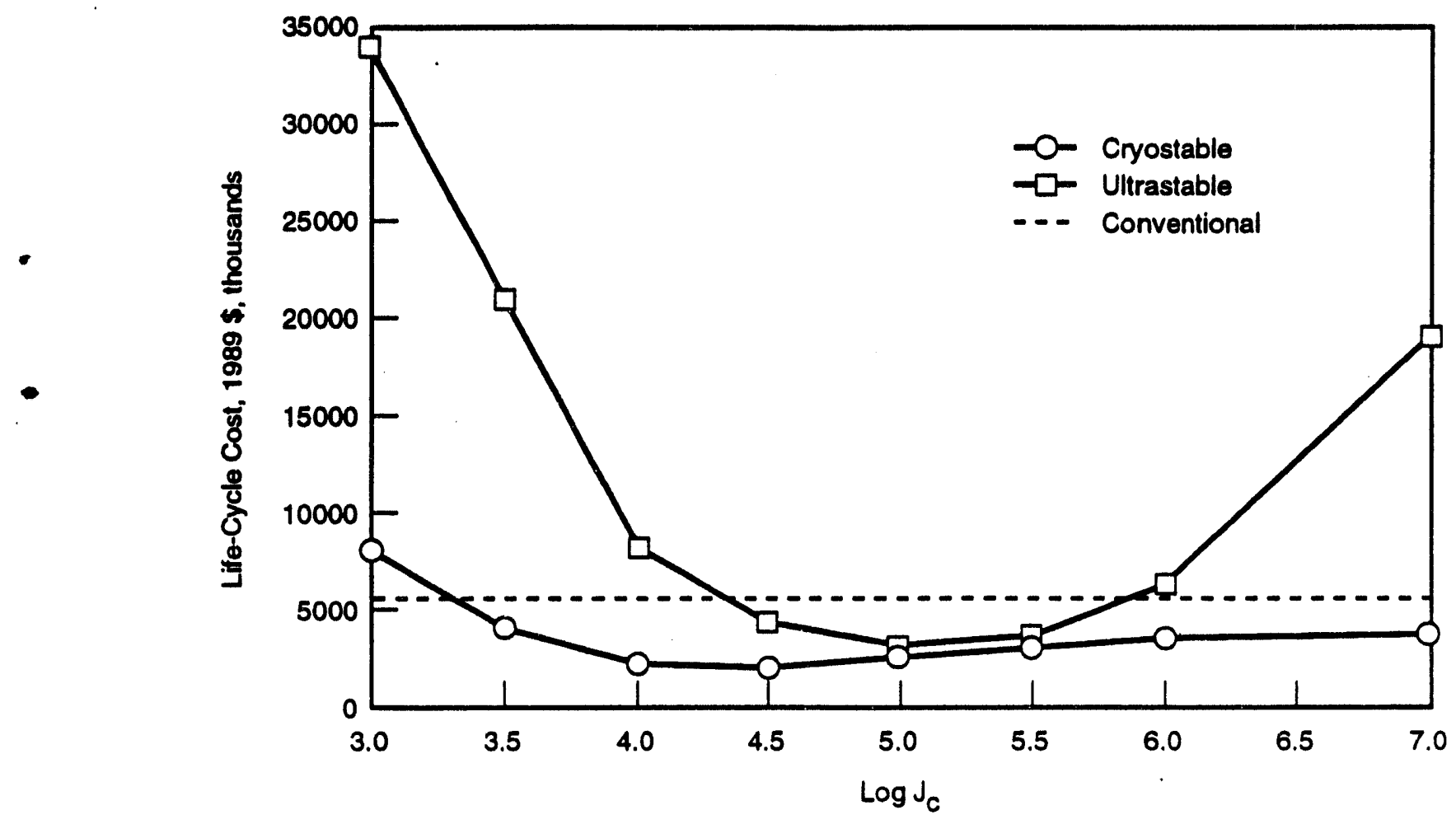

Figure 6.4. Impact of Various $\mathrm{J}_{\mathrm{c}}$ Assumptions on Transformer Life-Cycle Costs (1000-MVA Autotransformers)

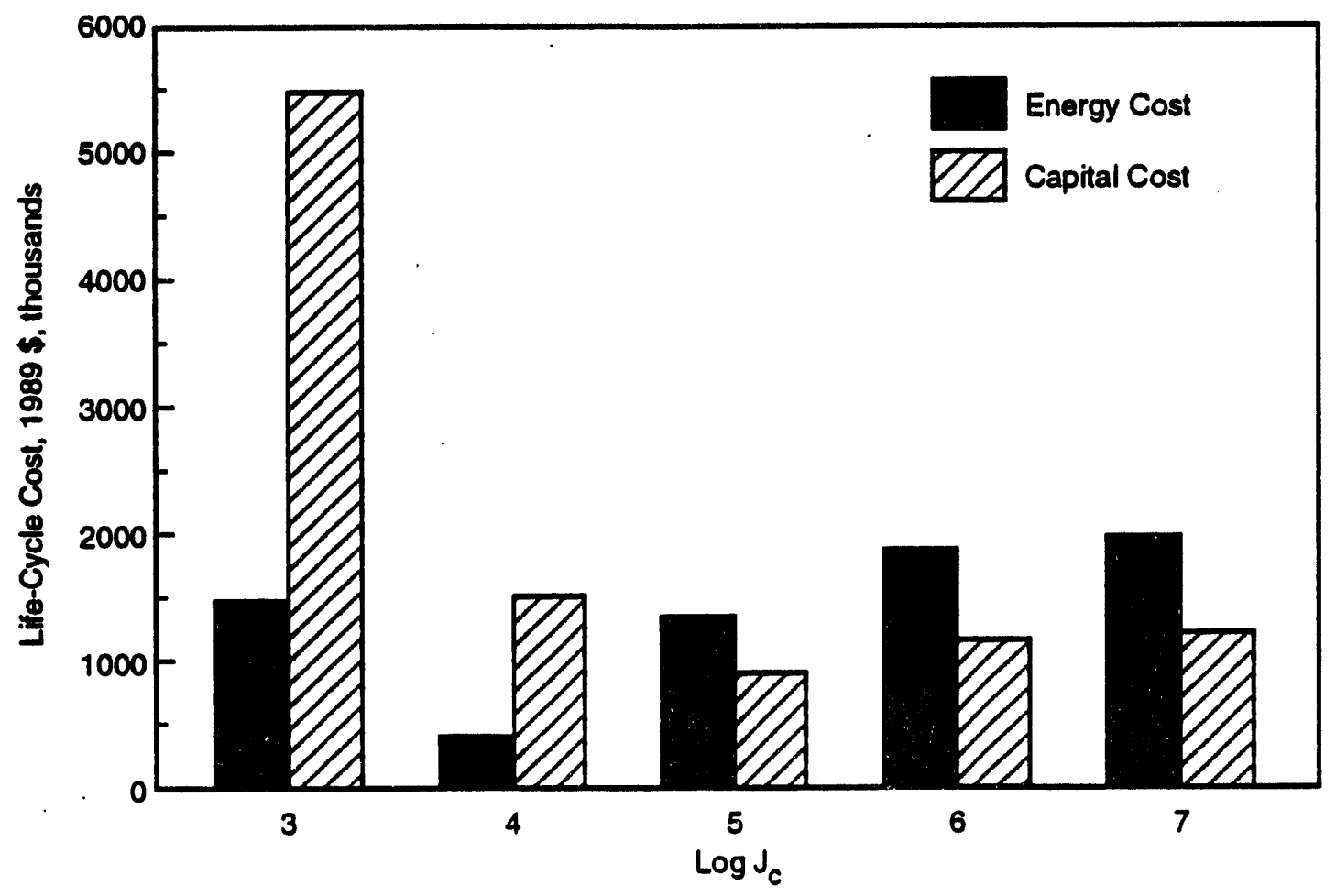

Figure 6.5. Impact of $J_{o}$ on HTS-Cryostable Life-Cycle Capital and Energy Cost (1000-MVA Autotransformers) 
these tradeoffs is that above a critical current density of about $10^{4.5} \mathrm{~A} / \mathrm{cm}^{2}$, the amount of copper is relatively constant, with the fraction of superconductor in the coil decreasing nearly proportionally with $\mathbf{J}_{\mathbf{c}}$.

The hysteresis losses in the cryostable superconductor are not a significant driver of the optimization process. The losses increase directly because of the increase in $J_{c}$, but remain relatively stable for critical currents of $10^{5} \mathrm{~A} / \mathrm{cm}^{2}$ and above, because the amount of superconductor material falls off sharply.

The heat leak losses (a function of the overall transformer size) and the stray magnetic losses (a function of the core losses and flux density) are relatively insensitive to $J_{c}$. These terms play a very minor role in the sensitivity analysis.

\subsubsection{Sensitivity Analysis with $\mathrm{J}_{\mathrm{c}}$ for Ultrastable Transformer Designs}

The critical current density has a very significant impact on the viability of the ultrastable design. In an effort to combat the high eddy-current losses found in the cryostable design, we investigated transformer designs that use redundant superconductive material rather than stabilizer metal to ensure stability. Eliminating the copper requirements in the conductor thereby eliminates eddy-current losses in the windings. However, the elimination of eddy-current loss in the windings is accompanied by increased hysteresis loss in the superconductor. Because the superconductor hysteresis loss per unit volume is dependent primarily on the magnetic field intensity to which the conductor is exposed, the redundant superconductor in the ultrastable transformer design has increased hysteresis losses in proportion to the amount of additional superconducting material.

Figure 6.6 shows the power losses for a family of ultrastable designs that have been optimized to minimize life-cycle costs at the respective critical current densities. For ease of comparison, the scales from Figure 6.1 have been used. At low values of $J_{c}$, the amount of superconducting material in the transformer windings is the primary driving factor in the optimization process. At higher critical current densities, the hysteresis loss becomes the main driver of the transformer design/ optimization process, with the loss becoming a very significant portion of the total losses for $\mathrm{J}_{\mathrm{c}}$ above $10^{4.5} \mathrm{~A} / \mathrm{cm}^{2}$.

Many of the loss components in the superconducting transformer are strongly influenced by changes in various design aspects of the transformer as the critical current density is varied.

Table 6.2 contains a summary of some of the important design results affected by changes in $J_{c}$ for optimized 1000-MVA ultrastable transformer designs. These will be discussed in the following sections. To better understand the processes involved, the critical current densities are again divided into three $\mathrm{J}_{\mathrm{c}}$ categories: low, intermediate, and high.

\subsubsection{Ultrastable Transformer Designs at Low $J_{c}$}

The winding conductor cross section is very large when $\mathrm{J}_{\mathrm{c}}$ is small. This is accented by the fact that there is a safety margin present to ensure stability, usually $20: 1$ as discussed in Section 3.3.1.2. For example: at $\mathrm{J}_{\mathrm{c}}=10^{3} \mathrm{~A} / \mathrm{cm}^{2}$, and a 1000-MVA, 230-kV autotransformer winding, the peak 


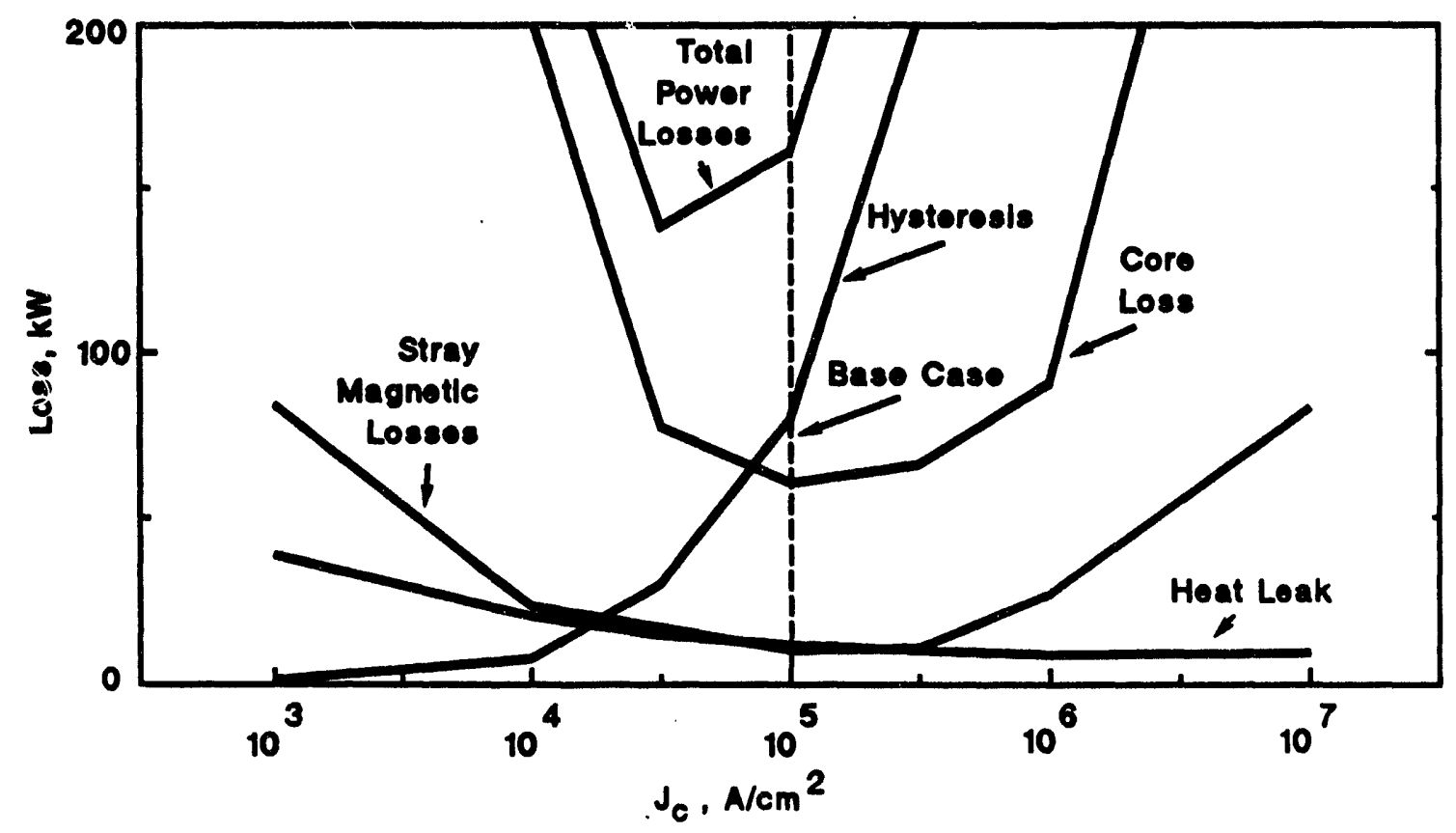

Figure 6.6. Ultrastable Transformer Individual Power Losses Versus Critical Currert Density $\left(\mathrm{J}_{\mathrm{c}}\right)$ (1000-MVA Autotransformers)

Table 6.2. Key Design Results for 1000-MVA Ultrastable Autotransformers as a Function of $J_{c}$

\begin{tabular}{|c|c|c|c|c|c|c|c|}
\hline \multirow[b]{2}{*}{ ملـ. } & \multicolumn{3}{|c|}{ Material Mass. ke } & \multicolumn{2}{|c|}{$\begin{array}{c}\text { Average Winding } \\
\text { Fields } \\
\end{array}$} & \multicolumn{2}{|c|}{ Conductor Radii } \\
\hline & Core & Cu & $S C$ & $\mathbf{B}_{1,1} \mathbf{G}$ & $B_{12, G}$ & $\mathbf{R}$ inol $\mathrm{mm}$ & $\mathbf{R}_{\text {imeen }} \mathrm{mm}$ \\
\hline 3 & 731,700 & 0 & 67,800 & 0.923 & 0.923 & 32.28 & 34.90 \\
\hline 4 & 184,400 & 0 & 10,590 & 6.058 & 6.058 & 10.21 & 11.04 \\
\hline 4.5 & 58,400 & 0 & 5,034 & 18.51 & 18.51 & 5.741 & 6.206 \\
\hline 5 & 50,080 & 0 & 1,533 & 56.23 & 56.23 & 3.228 & 3.490 \\
\hline 5.5 & 54,470 & 0 & 427.8 & 176.3 & 176.3 & 1.815 & 1.962 \\
\hline 6 & 62,390 & 0 & 114.8 & 556.7 & 556.7 & 1.021 & 1.104 \\
\hline 7 & 294,600 & 0 & 6.485 & 3587.0 & 3877.0 & 0.343 & 0.349 \\
\hline
\end{tabular}

operating current density in the winding is only $50 \mathrm{~A} / \mathrm{cm}^{2}$, which is much smaller than a conventional transformer, where current densities of approximately $300 \mathrm{~A} / \mathrm{cm}^{2}$ are common. The low current density in the conductors yields very bulky windings, which require large-core window areas. Therefore, the number of turns in the winding is severely curtailed by spatial requirements. The large amount of superconducting material required for low $\mathrm{J}_{\mathrm{o}}$ ultrastable transformer designs carries a very high capital cost. Because the number of turns in the optimum ultrastable transformer design is 
required to be kept small, Faraday's law indicates that the flux linked by the windings must be increased. Because the flux density cannot be increased beyond the capability of the core material, the necessary flux is established by increasing the cross-sectional area of the core. Therefore, the core must be relatively large for a low-J $\mathrm{J}_{\mathrm{c}}$ ultrastable-transformer design.

The combination of a relatively large transformer core and bulky winding yields designs with large dimensions. The necessarily large transformer volume for designs using low $\rrbracket_{c}$ superconductors causes penalties in other areas as well. An increased cryostat surface area allows higher thermal leakage into the cooled environment of the windings, and higher transformer volume yields increased leakage loss caused by stray magnetic flux. Overall, optimized low $\mathrm{J}_{\mathrm{o}}$ transformer designs are less efficient and more expensive than corresponding cryostable designs, as shown in Figures 6.2 and 6.3.

\subsubsection{Ultrastable Transformer Designs at Intermediate $\mathbf{J}_{\mathbf{c}}$}

As $\mathrm{J}_{\mathrm{c}}$ increases into the intermediate range (near $10^{5} \mathrm{~A} / \mathrm{cm}^{2}$ ), the winding conductors carry the load current more effectively. The peak operating current density (with a safety factor of 20) is now $5000 \mathrm{~A} / \mathrm{cm}^{2}$, which is at least an order of magnitude higher than that found in conventional transformer designs. This yields a more compact and lighter design than is possible with a comparable conventional transformer. However, this increase in $\mathrm{J}_{\mathrm{c}}$ results in higher hysteresis loss from an increased magnetic self-field. At intermediate critical current densities, the hysteresis loss becomes the dominant loss term (when the COP penalty is included to remove the heat generated from the cryogenic operating environment).

For intermediate $\mathrm{J}_{\mathrm{c}}$, the transformer size is relatively small (compared with conventional transformers), and the losses are at the minimum attainable range for minimum life-cycle cost ultrastable transformer designs. Thus, optimized ultrastable transformer designs tend to be more efficient than corresponding cryostable designs at intermediate values of $\mathrm{J}_{\mathrm{c}}$, as shown in Figure 6.2. Due to the moderate values of losses determined in the analysis and the relatively high capital expense of the superconducting material used in the windings, the ultrastable transformer designs are still somewhat more expensive than comparable cryostable designs that contain an order of magnitude less superconductive material.

\subsubsection{Ultrastable Transformer Designs at High $\mathbf{J}_{\mathbf{c}}$}

As $J_{c}$ is increased beyond $10^{5} \mathrm{~A} / \mathrm{cm}^{2}$, the hysteresis losses increase dramatically. These high hysteresis losses occur primarily because of the interaction of two factors:

- Hysteresis loss is dependent on $\mathrm{J}_{\mathrm{c}}$, with increasing $\mathrm{J}_{\mathrm{c}}$ leading to higher specific power loss for the superconductive material.

- The winding magnetic field intensity, largely driven by the self-field, increases substantially as the wire cross section is reduced. Therefore, an increase in $\mathrm{J}_{\mathrm{c}}$ leads to a more compact conductor, which increases the self-induced magnetic field, leading to higher hysteresis loss. The benefits of reduced self-field from stranding the conductor are eliminated at $\mathrm{J}_{\mathrm{c}}$ of $10^{7} \mathrm{~A} / \mathrm{cm}^{2}$ because the conductor radius becomes less than the strand radius of $0.5 \mathrm{~mm}$ assumed for all the other designs. 
Both of these factors are amplified because of the redundant superconductor present for satisfying the stability requirements. If the active current density of the conductor could be closer to the critical current density, the hysteresis loss would not be nearly as prominent.

Because the hysteresis loss increases so rapidly for these high $J_{c}$ values, the optimization process will limit the superconductor by curtailing the number of winding turns. Once again, to maintain the winding voltage, a reduction in the number of turns must be accompanied by an increase in the core magnetic flux. This can be achieved by increasing the flux density or by increasing the core crosssectional area. Because the flux density is near saturation, incremental increases in flux are obtained by increasing the core leg radius. This increase in core volume yields substantially increased core losses, which also increases the stray magnetic losses. The reason that the thermal leakage losses do not increase with the substantial increase in the core volume is that the cryostat surface area is not drastically affected by changes in core volume. The primary heat leak component is the conductive heat leak through the transformer bushings, which is modeled as being constant throughout the entire range of $J_{c}$ considered.

\subsubsection{Life-Cycle Cost Impacts for the Ultrastable Transformer Designs}

The sensitivity analyses on the life-cycle cost impact of $J_{c}$ for the ultrastable design were conducted using the same approach as applied in the cryostable case. Overall life-cycle costs as a function of $J_{c}$ were shown in Figure 6.4. A breakdown of the capital and energy loss portions of the life-cycle cost is shown in Figure 6.7. At a $J_{c}$ of $10^{3}$, the capital costs are quite high because of the large materials requirements, and the transformer core losses result in a large value of energy losses. As $J_{c}$ is increased, the transformer size and energy losses both decrease rapidly until a value of $J_{c}$ around $10^{4}$. At higher values of $\mathrm{J}_{\mathrm{c}}$, the hysteresis losses begin to rise rapidly. The result is an increase in the energy losses and the transformer capital costs as the optimal transformer design changes in an attempt to minimize the impacts of increasing hysteresis losses.

\subsubsection{Summary of $\mathrm{J}_{\mathrm{c}}$ Sensitivity Analysis for Ultrastable Designs}

The ultrastable transformer designs are very sensitive to changes in $J_{c}$. In fact, from the power loss standpoint, the optimum $J_{c}$ seems to lie near $10^{5} \mathrm{~A} / \mathrm{cm}^{2}$. We found that for lower values of $J_{c}$, the effective current density (when including the stability operating margin) was much less than a conventional transformer, thereby burdening the design with a very large core and associated core losses. For $\mathrm{J}_{\mathrm{c}}$ greater than $10^{5} \mathrm{~A} / \mathrm{cm}^{2}$, we found that the hysteresis loss became so great that the power losses would dictate that the amount of superconductor used in the windings be curtailed to keep the total within reasonable limits. This also led to large cores and respectively high losses.

The primary driver behind these hysteresis losses is $J_{c}$ itself. The effect of increasing $J_{c}$ was somewhat offset by the reduction in material required because of higher current densities, but the increased self-field from the smaller conductors contributed significantly to the losses. It is possible to design transformer with interleaved windings or using special conductors (such as Litz wire) to limit the magnitude of the self-field, but these were not included in the analysis. Also, the hysteresis loss equation, which is based on experience with low-temperature metal superconductors, may not be representative of fully developed high-temperature ceramic superconductors. If this were the case, 


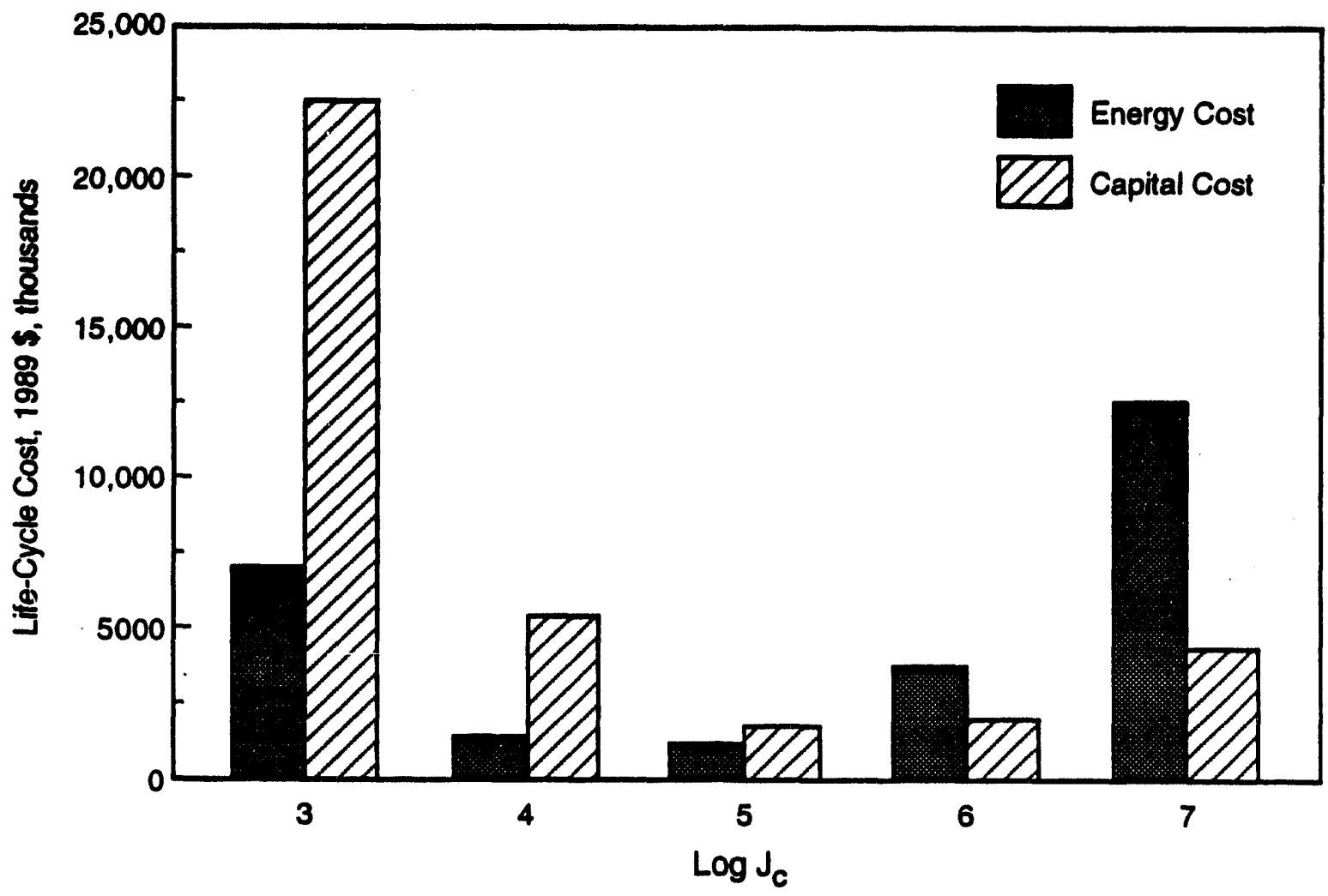

Figure 6.7. Impact of $J_{c}$ on HTS-Ultrastable Life-Cycle Capital and Energy Cost (1000-MVA Autotransformers)

new determinations of hysteresis loss would need to be made to fully understand the interactions found in a detailed transformer design process.

\subsubsection{Critical Current Density Sensitivity Analysis Conclusions}

Our investigations yield insight as to the predicted range of $J_{c}$ that could yield viable transformer designs, as well as the relative impact of advances of material performance on transformer performance. Comparisons of the efficiency and overall transformer life-cycle cost between cryostable and ultrastable designs as a function of $\mathrm{J}_{\mathrm{c}}$ may be made from Figures 6.2 and 6.3.

The impact and importance of high $\mathrm{J}_{\mathrm{c}}$ levels on the transformer life-cycle cost varies significantly for the two HTS transformer designs evaluated. These results are shown in Figure 6.4, Section 6.1.2. The results indicate that there is an optimal $J_{c}$ for both types of transformers and that the impact of $J_{c}$ is very different for the two design approaches. For the ultrastable design, the impact of $J_{c}$ is profound, with a sharp optimal region. The results suggest that the minimum $J_{c}$ required for an ultrastable design to build transformers that are economically superior to conventional transformers is somewhere above $10^{4} \mathrm{~A} / \mathrm{cm}^{2}$. In comparison, the impact of $J_{c}$ on the life-cycle cost 
design is less pronounced; the results indicate that a cryostable transformer could be economically superior to a conventional transformer with a minimum $\mathrm{J}_{\mathrm{c}}$ around $10^{3} \mathrm{~A} / \mathrm{cm}^{2}$.

For an ultrastable transformer to compete with a conventional transformer, the critical current density of the material should be at least $10^{4} \mathrm{~A} / \mathrm{cm}^{2}$. The viability appears to become better for slightly higher values of $J_{c}$, but beyond $J_{c}=10^{5} \mathrm{~A} / \mathrm{Cm}^{2}$, we actually found decreasing benefits from use of such material. This suggests that high-temperature superconductor research efforts should first target the development of materials with $J_{c}$ less than $10^{6} \mathrm{~A} / \mathrm{cm}^{2}$ for power transformer applications.

The exact location of the knee in the performance and life-cycle cost curves is dependent primarily on the fields to which the conductors are exposed (see Tables 6.1 and 6.2). Thus, if the self-fields determined by the model are not representative of actual designs that would be constructed in the future (which may, in fact, be designed with the intent of minimizing the negative effects of high self-fields), the location of the knee would be different. While it is likely that a knee will always exist at some $J_{c}$, good engineering design may permit shifting the knee to a higher $J_{c}$ than can possibly be attained with high-temperature superconductors. Therefore, by considering only attainable values of $\mathrm{J}_{c}$, it would appear that further increases in $\mathrm{J}_{c}$, combined with good engineering design, will always result in further increases in efficiency and decreases in life-cycle cost.

\subsection{Self-Field Sensitivity}

Because of the obvious importance of the self-field in determining the optimal design and ultimate performance of superconducting power transformers, a sensitivity analysis on self-fields was performed. This analysis demonstrates that relatively small changes in the self-field result in huge variations in the performance, materials requirements, and life-cycle costs.

The expression developed for determining the average self-field seen by the conductor is explained is Section 3.4.2. As mentioned earlier, the average field to which the conductors are exposed depends heavily on the transformer configuration, the winding geometry, the amount and placement of insulation, the arrangement of the conductors within the windings, the degree of stranding in the conductors, and other factors. Thus, field exposure of the windings on a microscopic level cannot be precisely calculated without a complex finite element analysis involving all of the above design factors for the myriad of potential transformer designs that must be evaluated.

Furthermore, the actual field exposure of the windings is a design problem and cannot be precisely known until a transformer is built and tested. Because the results of this study have shown that significant negative effects are caused by high self-fields, it is likely that any actual superconductive transformer design will consider these effects and attempt to minimize them. Possible methods of reducing the effects of high self-fields include designing transformers with interleaved windings or using special conductors (such as Litz wire) to limit the magnitude of the self-field.

Thus, we developed a simple model that reasonably approximates the average self-field to which the conductors in the transformer windings are exposed, and that accounts for both macroscopic and microscopic field effects. The expression for the self-field used in both our conventional and base 
case superconductive transformer design models is given by Equation (6.1), which is described in detail in Section 3.4.2.

Base case average self-field expression

$$
H_{s}=\frac{0.7 I}{2 \sqrt{2} \pi R}\left(\frac{r_{s}}{R}\right)
$$

The reasonable limits of the actual self-field include at one end the self-field for a single cylindrical conductor in free space (assumes no cancellation) and, at the other end of the possible self-field spectrum, the zero self-field (assumes complete cancellation). The average self-induced magnetic field is given by Equation (6.2):

No cancellation average self-field expression

$$
H_{s}=\frac{I}{2 \sqrt{2} \pi R}
$$

The average self-induced magnetic field assuming complete cancellation is given by the following expression:

$$
\begin{aligned}
& \text { Complete cancellation (no self-field) expression } \\
& \qquad H_{s}=0
\end{aligned}
$$

To evaluate how the design, performance, materials, requirements, and life-sycle costs are affected by the different self-field equations, optimal designs were determined for transformers having the same design specifications (1000-MVA, 500/230-kV, $3 \phi$ autotransformer, conventional core), with corresponding variations in the self-field expression. Table 6.3 shows the key design results for both the cryostable and ultrastable superconductive designs.

Notice that the average windings fields (a combination of self-field and stray field) show large variations in magnitude, from a minimum near zero (no self-field) to a maximum of over 1000 gauss ( 0.1 tesla) in the cryostable design with no cancellation. This variation in the average self-field to which the conductors are exposed completely dominates and determines the optimal designs. The result is that at zero self-field, the designs tend to minimize the core material requirements (and associated losses) at the expense of increased superconductor (and stabilizer) requirements. However, because the average field to which these windings are exposed is small and, hence, so are the losses, these designs are highly efficient. The major losses are confined to the core, leakage, and cryostat heat leak categories (see Tables 6.4 and 6.5).

When very high self-fields are considered (the no-cancellation case), the effect is the opposite. Every effort is made to minimize the amount of superconductor and stabilizer (and their associated losses). By increasing the amount of material in the transformer core, relatively fewer turns are required in the superconductive transformer windings. An increase in the core volume does result in increased core losses; however, the winding volume with its associated relatively high material cost and high losses is minimized. The resulting designs have a very large materials requirement 
Table 6.3. Key Design Results for Self-Field Sensitivity (1000-MVA Autotransformers)

\begin{tabular}{|c|c|c|c|c|c|c|c|c|}
\hline \multirow[b]{2}{*}{$\begin{array}{l}\text { Design } \\
\text { Basis } \\
\end{array}$} & \multirow[b]{2}{*}{$\begin{array}{l}\text { Core Flux } \\
\text { Density. T }\end{array}$} & \multicolumn{3}{|c|}{ Material Mass, kg } & \multicolumn{2}{|c|}{$\begin{array}{c}\text { Average Winding } \\
\text { Fields }\end{array}$} & \multicolumn{2}{|c|}{ Conductor Radii } \\
\hline & & Core & $\mathrm{Cu}$ & SC & $\underline{B}_{L 1} \cdot G$ & $\mathrm{~B}_{\mathrm{L} 2, \mathrm{G}}$ & $\begin{array}{c}R_{\text {wirol }}, \\
\mathrm{mm}\end{array}$ & $\begin{array}{c}\mathbf{R}_{\text {wire2 }} \\
\mathrm{mm}\end{array}$ \\
\hline \multicolumn{9}{|l|}{ Cryostable } \\
\hline No field & 1.50 & 9,283 & 1,209 & 333.7 & 0.690 & 0.690 & 1.672 & 1.791 \\
\hline Base case & 1.60 & 24,360 & 655.6 & 181.1 & 220.0 & 212.1 & 1.672 & 1.791 \\
\hline No cancel & 1.50 & 232,700 & 270.7 & 74.83 & 1006.9 & 1068.7 & 1.672 & 1.791 \\
\hline \multicolumn{9}{|l|}{ Ultrastable } \\
\hline No field & 1.60 & 22,770 & 0 & 2,234 & 1.295 & 1.295 & 3.228 & 3.490 \\
\hline Base case & 1.50 & 50,080 & 0 & 1,533 & 56.23 & 56.23 & 3.228 & 3.490 \\
\hline No cancel & 1.50 & 115,200 & 0 & 1,036 & 507.8 & 548.9 & 3.228 & 3.490 \\
\hline
\end{tabular}

(Table 6.3) and poor efficiencies. Even with the optimal designs attempting to limit losses in the windings (hysteresis losses in the superconductor and eddy-current losses in the stability material), the windings losses represent over $70 \%$ of the total losses.

In conclusion, for superconducting transformers to be more efficient and cost-effective (see Tables 6.4, 6.5 and 6.6) than conventional designs, great care must be exercised in designing transformers that will minimize the fields to which the conductors are exposed. Thus, leakage and self-field minimization is likely to be the single most important factor in the design and ultimate performance of superconducting transformers and other ac superconducting devices. This issue can be addressed through a detailed design and analysis, but is likely to be resolved only when an HTS transformer is constructed and tested.

\subsection{Filament Diameter Sensitivity}

The impact of the HTS filament diameter is another example of the type of investigations possible with the models developed in this study. A different filament diameter assumption changes the calculated transformer losses. This results in a new optimal transformer design with a different life-cycle cost. Investigations of this type allow insights into the interaction between materials characteristics, design parameters, cost, and market penetration.

As mentioned throughout this document, the superconducting material parameters tend to drive the design/performance of the transformers. For all applications using the base case material parameters, the cryostable designs had lower life-cycle costs than the ultrastable designs. However, this is largely the result of the choice of the base case material parameters (see Table 3.2). The base 
Table 6.4. Performance Results for Self-Field Sensitivity-Cryostable--1000-MVA Autotransformers

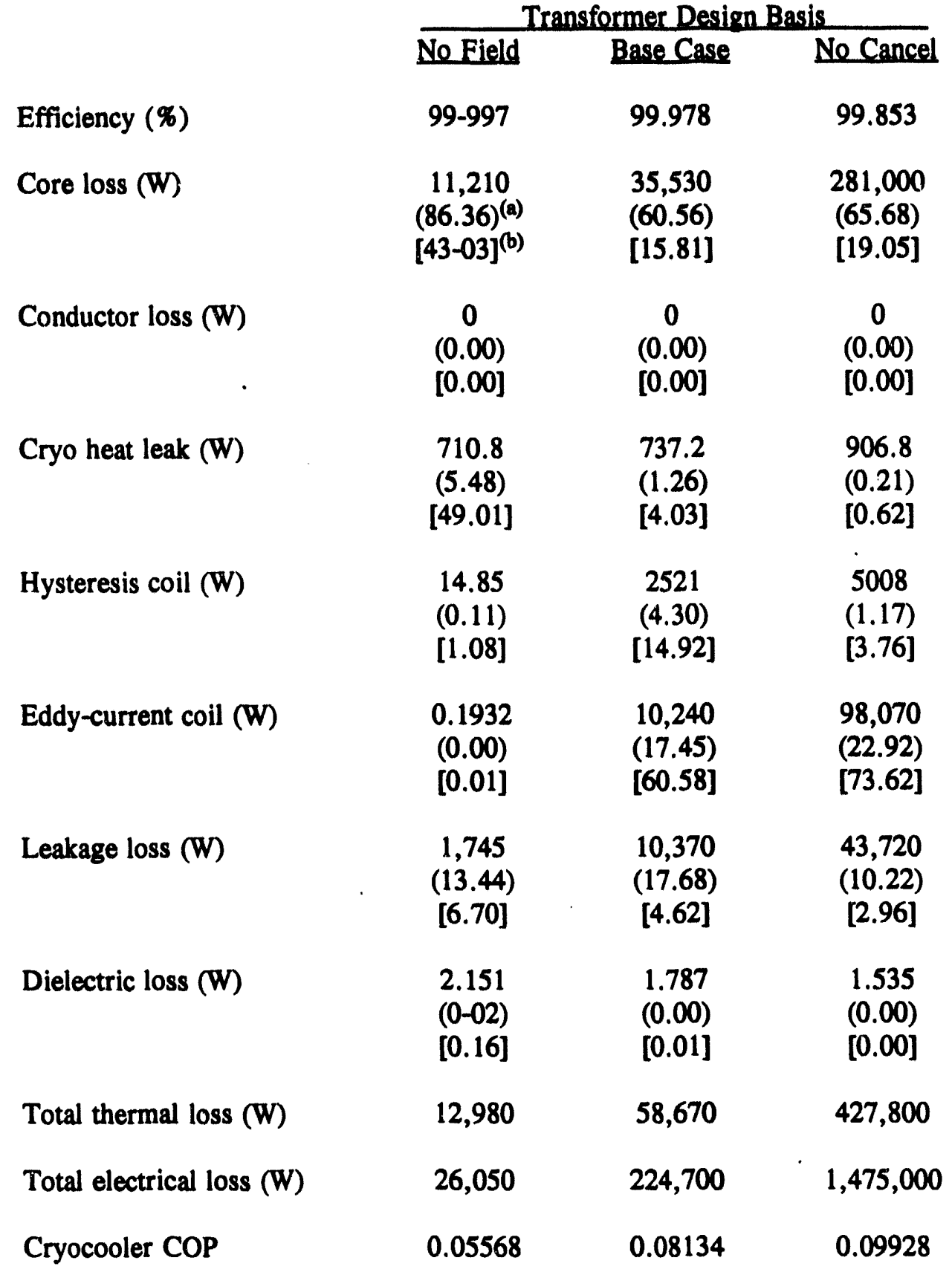

(a) Percentage of total thermal losses shown in parentheses.

(b) Percentage of total electric losses shown in square brackets. 
Table 6.5. Performance Results for Self-Field Sensitivity-Ultrastable--1000-MVA Autotransformers

\begin{tabular}{|c|c|c|c|}
\hline & \multicolumn{3}{|c|}{ Transformer Desien Basis } \\
\hline & Ne Field & Base Case & No Cancel \\
\hline Efficiency (\%) & 99.994 & 99.984 & 99.940 \\
\hline Core loss (W) & $\begin{array}{c}33,210 \\
(77.05)^{(a)} \\
{[55.31]^{(b)}}\end{array}$ & $\begin{array}{l}60,490 \\
(80.16) \\
{[37.57]}\end{array}$ & $\begin{array}{l}139,100 \\
(70.93) \\
{[23.25]}\end{array}$ \\
\hline Conductor loss (W) & $\begin{array}{c}0 \\
(0.00) \\
{[0.00]}\end{array}$ & $\begin{array}{c}0 \\
(0.00) \\
{[0.00]}\end{array}$ & $\begin{array}{c}0 \\
(0.00) \\
{[0.00]}\end{array}$ \\
\hline Cryo heat leak (W) & $\begin{array}{c}799.6 \\
(1.86) \\
{[22.82]}\end{array}$ & $\begin{array}{l}836.0 \\
(1.11) \\
{[6.94]}\end{array}$ & $\begin{array}{l}895.1 \\
(0.46) \\
{[1.66]}\end{array}$ \\
\hline Hysteresis coil (W) & $\begin{array}{l}186.5 \\
(0.43) \\
{[5.63]}\end{array}$ & $\begin{array}{c}5,557 \\
(7.36) \\
{[49.61]}\end{array}$ & $\begin{array}{l}35,300 \\
(18.00) \\
{[71.46]}\end{array}$ \\
\hline Eddy-current coil (W) & $\begin{array}{c}0 \\
(0.00) \\
{[0.00]}\end{array}$ & $\begin{array}{c}0 \\
(0.00) \\
{[0.00]}\end{array}$ & $\begin{array}{c}0 \\
(0.00) \\
{[0.00]}\end{array}$ \\
\hline Leakage loss (W) & $\begin{array}{c}9,694 \\
(22.49) \\
{[16.15]}\end{array}$ & $\begin{array}{c}9,411 \\
(12.47) \\
{[5.85]}\end{array}$ & $\begin{array}{c}21,640 \\
(11.04) \\
{[3.62]}\end{array}$ \\
\hline Dielectric loss (W) & $\begin{array}{l}2.583 \\
(0.01) \\
{[0.08]}\end{array}$ & $\begin{array}{l}2.451 \\
(0.00) \\
{[0.02]}\end{array}$ & $\begin{array}{l}2.408 \\
(0.00) \\
{[0.00]}\end{array}$ \\
\hline Total thermal loss (W) & 43,100 & 75,460 & 196,100 \\
\hline Total electrical loss (W) & 60,040 & 161,000 & 598,300 \\
\hline Cryocooler COP & 0.05837 & 0.07477 & 0.09000 \\
\hline
\end{tabular}

(a) Percentage of total thermal losses shown in parentheses.

(b) Percentage of total electric losses shown in square brackets. 
Table 6.6. Life-Cycle Cost Results for Self-Field Sensitivity Analysis (1000-MVA Autotransformers)

$\begin{array}{lc}\text { Desien Basis } & \begin{array}{c}\text { Life-Cycle C } \\ 1989 \\ \text { Sryostable }\end{array} \\ \text { No field } & 742 \\ \text { Base case } & 2,434 \\ \text { No cancel } & 13,698 \\ \text { Ultrastable } & \\ \text { No field } & \\ \text { Base case } & 2,104 \\ \text { No cancel } & 3,009 \\ \end{array}$

case assumption for the HTS filament diameter is $50 \mu \mathrm{m}$. In this sensitivity analysis, we explored the effect of a significant decrease in the filament diameter.

The filament diameter of the HTS material impacts the design of HTS transformers through the calculation of the hysteresis losses in the superconducting material. Hysteresis losses are linearly related to the filament diameter [see Equation (3.16)]. Because the design and performance of the crystable transformers are driven largely by the eddy-current losses in the stability material (which are not affected by the filament diameter), these designs show only a slight improvement in performance with a change in the filament diameter. However, the ultrastable design and performance are dominated by the hysteresis loss. Hence, a significant decrease in the filament diameter has a dramatic effect on the ultrastable designs. Table 6.7 shows how the filament diameter influences the full-load losses of 1000-MVA autotransformers with conventional cores.

Not only are the losses profoundly decreased for the ultrastable transformers, but the design is also significantly changed because the optimization process no longer tries to minimize the hysteresis losses to the same extent (e.g., more HTS material is used, and a significantly smaller core is possible). Figure 6.8 illustrates the effect on life-cycle costs of changing the superconductive filament diameter in 1000-MVA HTS autotransformers.

This sensitivity analysis is an example of the dramatic effect that a change in only one material parameter can have on the comparison of the two types of HTS transformers. Sensitivity analyses such as these can be used to help define the necessary HTS material requirements for superconducting transformers that are economically superior to conventional designs. These analyses also will provide input to the development of national goals for HTS material. 
Table 6.7. Full-Load Loss Results for Filament Diameter Sensitivity Analysis (1000-MVA Autotransformers)

Design Basis

Filament Diameter

Cryostable

Base case $50 \mu \mathrm{m}$

Sensitivity $5 \mu \mathrm{m}$

Sensitivity $0.5 \mu \mathrm{m}$

Ultrastable

Base case $50 \mu \mathrm{m}$

Sensitivity $5 \mu \mathrm{m}$

Sensitivity $0.5 \mu \mathrm{m}$

\section{Eull-Load Loss. $k W$}

224.6

197.3

194.5

161.0

69.6

58.4

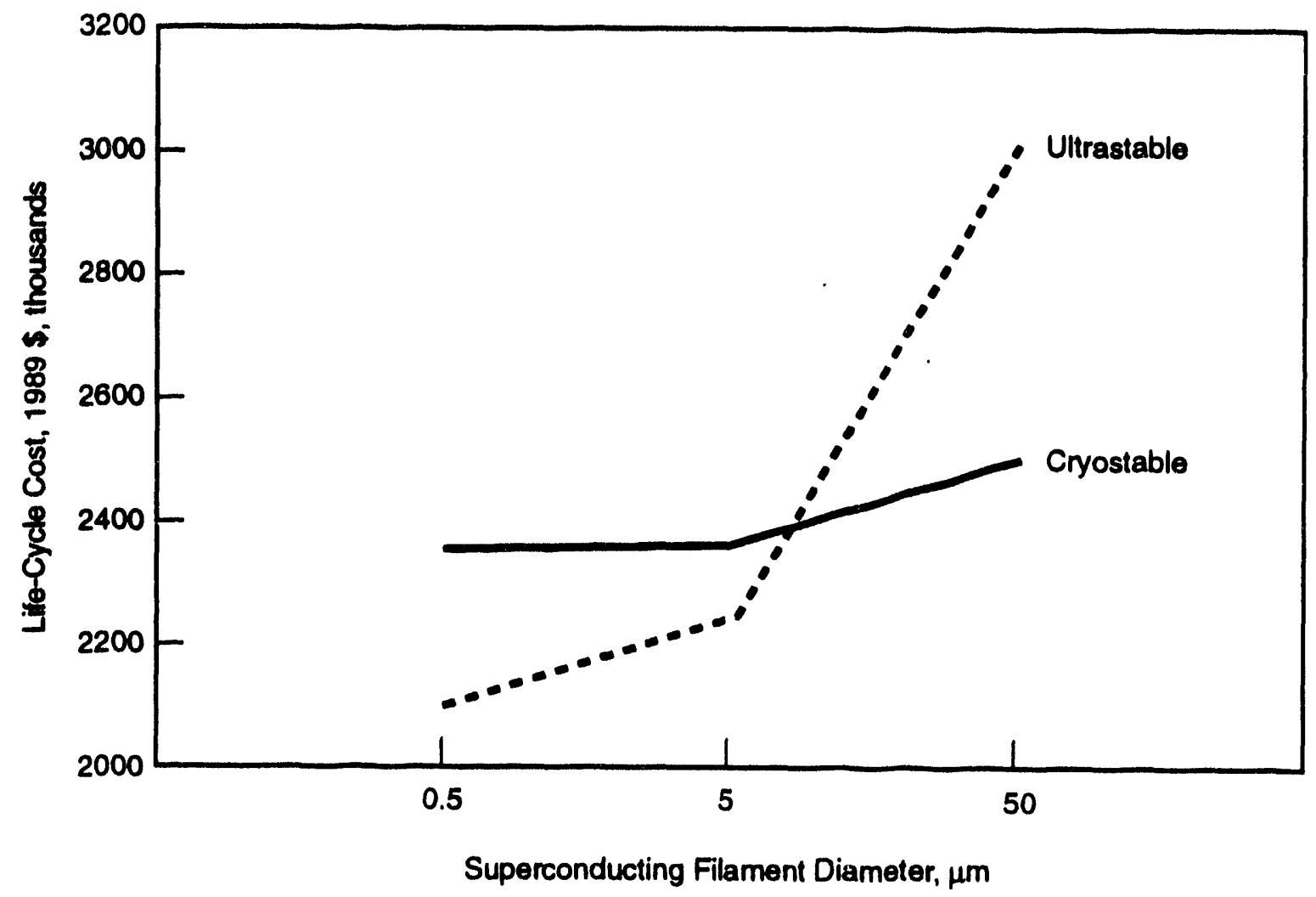

Figure 6.8. Effect of Superconductive Filament Diameter on Life-Cycle Costs (1000-MVA Autotransformers) 


\subsection{Sensitivity Analysis Conclusions}

The key conclusions we have developed to date from the sensitivity analysis are the following:

- For the base case materials parameters, the HTS-CS technology appears to be economically superior to the HTS-US technology at values of $J_{c}$ below about $10^{5} \mathrm{~A} / \mathrm{cm}^{2}$.

- The results of this study indicate that very high levels of $J_{c}$ should not be required for designing a cryostable transformer economically superior to a conventional transformer. For the economic conditions assumed in this study, it appears possible to design cost-effective cryostable transformers at values of $J_{c}$ less than $10^{4} \mathrm{~A} / \mathrm{cm}^{2}$.

- For ultrastable transformers to compete with conventional transformers, the critical current density of the material should be at least $10^{4} \mathrm{~A} / \mathrm{cm}^{2}$. The viability appears to become better for slightly higher values of $J_{c}$, but beyond $J_{c}=10^{5} \mathrm{~A} / \mathrm{cm}^{2}$ we actually found decreasing benefits from using such material. This suggests that high-temperature superconductor research efforts should first target the development of materials with $\mathrm{J}_{\mathrm{c}}$ less than $10^{6} \mathrm{~A} / \mathrm{cm}^{2}$ for power transformer applications.

- The existence of a knee in the performance and life-cycle cost curves, as a function of critical current density, depends primarily on the fields to which the conductors are exposed. Thus, if the self-fields determined ' $y$ the model are not representative of actual designs that would be constructed in the future (which may, in fact, be designed with the intent of minimizing the negative effects of high self-fields), the location of the knee would be different. While it is likely that a knee will always exist at some $J_{c}$, good engineering design may be able to shift the knee to a higher $\mathrm{J}_{\mathrm{c}}$ than can possibly be attained with high-temperature superconductors. Therefore, by considering only attainable values of $J_{c}$, it would appear that further increases in $J_{c}$ will always result in further increases in efficiency and decreases in life-cycle cost.

- For superconducting transformers to be more efficient and cost-effective than conventional designs, great care must be exercised in designing transformers that will minimize the fields to which the conductors are exposed. Minimization of the field (leakage or self-field) that the conductors are exposed to is likely to be the single most important factor in the design and ultimate performance of superconducting transformers and other ac superconducting devices. Final resolution of this issue requires detailed design and analysis, prototype testing, or both.

- The superconducting material parameters tend to drive the design and performance of the HTS transformers. By changing the assumed filament diameter of the HTS material from $50 \mu \mathrm{m}$ to $0.5 \mu \mathrm{m}$, we showed that the ultrastable design losses decreased by up to $64 \%$ and the life-cycle cost decreased by $307 \%$. The effect on the cryostable design was much less significant, with losses and life-cycle costs changing less than $15 \%$. Thus, it is impossible to say whether cryostable or ultrastable designs will have the ultimate cost and performance advantage until the material parameters are known. However, analyses such as these can point to areas where research will provide the most beneficial results. 


\subsection{Market Penetration and Benefits to the Nation}

In this chapter, we describe the methodology used to estimate potential sales of HTS transformers in the electric utility market and potential benefits to the nation from the use of these transformers.

The basic methodology begins by establishing the inventory of auto and step-up transformers as shown in Figure 7.1, box 1.0. [This inventory is based on electrical generating capacity and survey information collected by the National Electrical Manufacturers Association (NEMA).] Transformer replacement requirements are the result of failures, retirements (2.0), or load growth (3.0). Each of these requirements is estimated separately to generate an array of total (annual) transformer requirements (4.0). When a transformer need is identified, utilities must choose either a conventional or an HTS transformer. The selection process begins with the transformer market share calculation (5.0) that is based on a comparison of the relative life-cycle costs of the two transformer types. The market share calculation is then adjusted (6.0), based on the initial risk that the utility perceives is present in adopting a new technology, the utility's technical limitations, and its concern with higher HTS initial costs. After a transformer has been chosen (7.0), and conventional (8.0) and HTS (9.0) transformer sales are known, the benefits to the nation from adopting HTS transformers are estimated (10.0). The benefits include both the energy saved from adopting more efficient transformers and the monetary savings of adopting a transformer with a lower cost (in terms of life-cycle costs). Each of the steps in the methodology is described in detail in this chapter.

Four HTS transformer types were evaluated:

- ultrastable conventional core

- ultrastable amọrphous core

- cryostable conventional core

- cryostable amorphous core.

For each HTS transformer design, three autotransformers and three step-up transformers were compared to their conventional counterparts, to evaluate potential market performance. The results of this evaluation are presented at the end of this chapter.

\subsection{Development of Initial Transformer Inventory}

Because no data are available on the existing inventory of transformers, these data had to be generated based on other available information. One data source indicates that the United States has approximately 27,000 power transformers (not including pole-top distribution transformers) (Ivesom 1989; Feher et al. 1988). Because of the lack of data on the inventory of operating power 


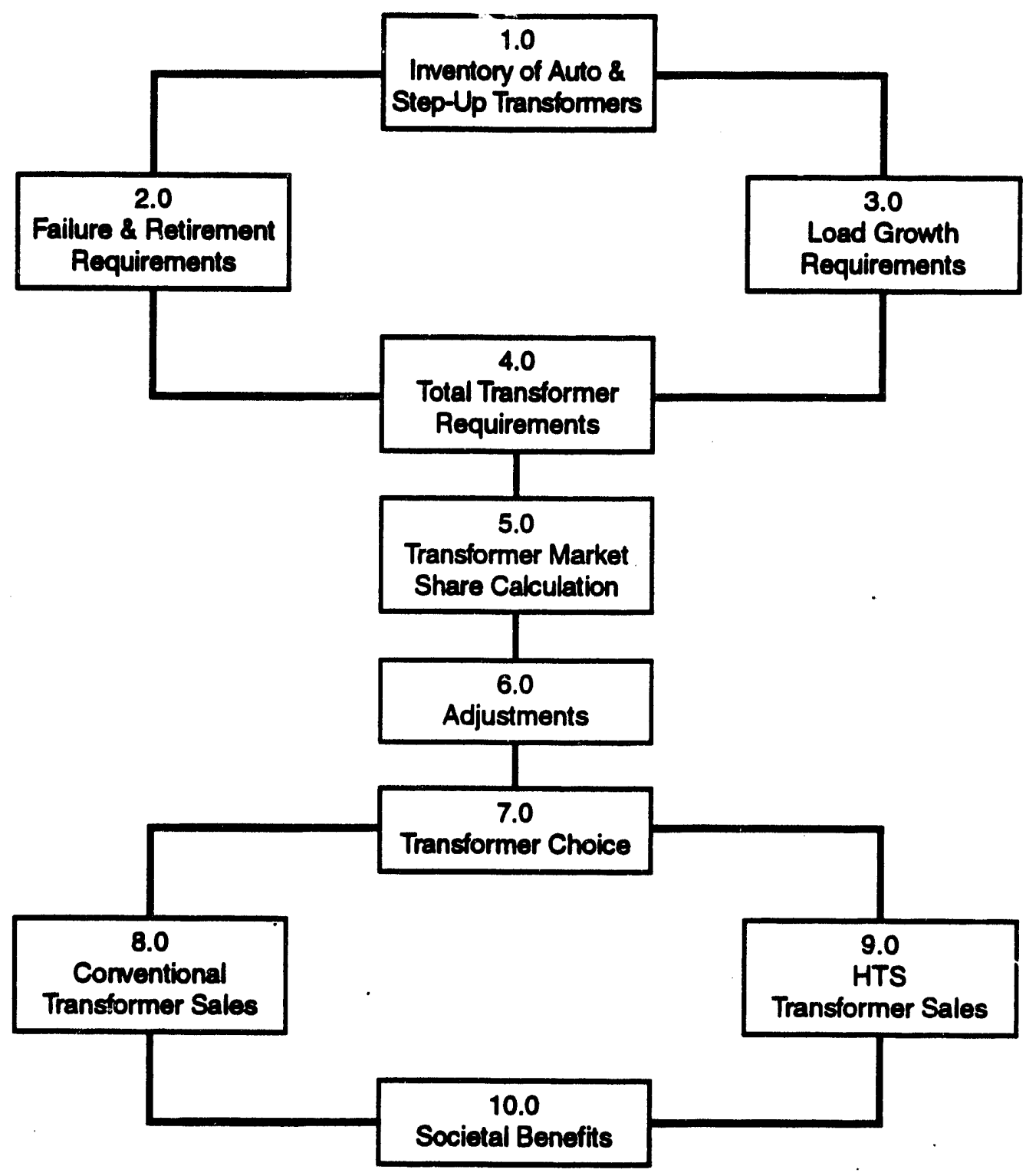

Figure 7.1. Methodology for Estimating Market Penetration and Benefits to the Nation

transformers, the initial inventory had to be generated from the history of generating capacity additions and the available NEMA data on transformer sales. To generate the inventory, we followed these steps:

1. The annual change in generating capacity was determined.

2. Annual generator installations (in megawatts) were estimated, including new additions and replacements of failed generators (and those replaced for economic reasons). 
3. From the estimated annual generator installations and the ratio of transformer sales (by type) to generator sales, the total megavolt-amperes (MVA) of transformers installed each year were determined by type.

4. From the MVA of each type of transformer model of the utility grid, the number of installed in each year was determined.

5. Using a Weibull distribution, the number of installed transformers still surviving by year was calculated. This is the initial transformer inventory.

\subsubsection{Annual Generating Capacity Changes}

Data on generating capacity were taken from the Annual Energy Review 1988 (EIA 1989, p. 209). The net summer capability [in gigawatts (GW) of capacity] of electric utilities, for the years 1949 through 1988 inclusive, was used to calculate the annual change in generating capacity. The annual change is simply the difference between the year-ending capacity in one year and the yearending capacity in the previous year; these values are presented in Table 7.1. Because the maximum transformer age is assumed to be $\mathbf{4 0}$ years, and because only $\mathbf{4 0}$ years of generating capacity information data are available (41 years of data are required for differencing), the annual change in generating capacity for 1948 to 1949 was assumed to be the same percentage change as that from 1949 to 1950.

\subsubsection{Annual Generator Installations}

No data are available on long-term projections of transformer sales by size and application. Thus, these projections were based on estimates of annual generator installations and relationships between generator installations and transformer sales. Generator installations are easier to track and project than transformer installations because EIA regularly gathers data on the nation's electrical generation capability (EIA 1989), and NEMA gathers data on the generating additions and retirements (NEMA 1986, 1974).

Annual generator installations are somewhat higher than the change in generation capability because not all generator installations represent increases in generation capability. Some generator installations are replacements for generators that are retired because of failures, economics, or other reasons. NEMA data on generator installations and retirements for the period 1957 through 1984 were used to determine an estimate of generator installations (NEMA 1986, 1974). According to the NEMA data, generator installations exceeded the net change in generation capability by $8.46 \%$ over this time period. Thus, actual gigawatts of generator installations, by year, were estimated to be $8.46 \%$ higher than the net change in summer generation capability, shown in the last column of Table 7.1.

\subsubsection{Total Transformer Installations Each Year}

From the estimated annual generator installations and the ratio of transformer sales to generator sales, the total MVA of transformers installed each year by type was determined. NEMA data on total generator installations (MW) and on total transformer installations (MVA) show major 
Table 7.1. Net Summer Generation Capability for Electric Utilities

\begin{tabular}{|c|c|c|}
\hline Year & $\begin{array}{l}\text { Net Summer Generation } \\
\text { Capability, GW }\end{array}$ & $\begin{array}{c}\text { Change in Net Summer } \\
\text { Generation Capability. GW }\end{array}$ \\
\hline 1949 & 63.4 & 5.3 \\
\hline 1950 & 69.2 & 5.8 \\
\hline 1951 & 75.5 & 6.3 \\
\hline 1952 & 83.2 & 7.7 \\
\hline 1953 & 93.3 & 10.1 \\
\hline 1954 & 100.0 & 6.7 \\
\hline 1955 & 114.2 & 14.2 \\
\hline 1956 & 119.7 & 5.5 \\
\hline 1957 & 131.1 & 11.4 \\
\hline 1958 & 143.3 & 12.2 \\
\hline 1959 & 155.9 & 12.6 \\
\hline 1960 & 167.1 & 11.2 \\
\hline 1961 & 179.0 & 11.9 \\
\hline 1962 & 192.1 & 13.1 \\
\hline 1963 & 209.7 & 17.6 \\
\hline 1964 & 223.7 & 14.0 \\
\hline 1965 & 234.8 & 11.1 \\
\hline 1966 & 247.5 & 12.7 \\
\hline 1967 & 266.7 & 19.2 \\
\hline 1968 & 284.0 & 17.3 \\
\hline 1969 & 309.8 & 25.8 \\
\hline 1970 & 336.4 & 26.6 \\
\hline 1971 & 366.4 & 30.0 \\
\hline 1972 & 396.0 & 29.6 \\
\hline 1973 & 439.8 & 43.8 \\
\hline 1974 & 468.5 & 28.7 \\
\hline 1975 & 491.3 & 22.8 \\
\hline 1976 & 517.2 & 25.9 \\
\hline 1977 & 535.9 & 18.7 \\
\hline 1979 & 552.1 & 16.2 \\
\hline 1979 & 565.5 & 13.4 \\
\hline 1980 & 578.6 & 13.1 \\
\hline 1981 & 598.1 & 19.5 \\
\hline 1982 & 613.7 & 15.6 \\
\hline 1983 & 621.1 & 7.4 \\
\hline 1984 & 635.1 & 14.0 \\
\hline 1985 & 655.2 & 20.1 \\
\hline 1986 & 664.8 & 9.6 \\
\hline 1987 & 674.1 & 9.3 \\
\hline 1988 & 678.4 & 4.3 \\
\hline
\end{tabular}

Source: Annual Energy Review 1988 (EIA 1989). 
inconsistencies between survey years. In addition, the NEMA-provided historical data are not sufficient to cover the necessary 40-year history. Thus, only data from the tenth biennial survey were used in determining the ratio of transformer installation to generator installations (NEMA 1986).

Ratios of transformer installations (MVA) to generator installations (MW) were determined from the NEMA data over the period 1976 through 1984. During this period, utilities installed 1.259 MVA of main generator transformers, 2.168 MVA of autotransformers, and 1.072 MVA of other transformers for every MW of generators installed. The "other transformers" are usually step-down units feeding into load buses from the transmission system (NEMA 1986). Thus, about 4.5 MVA of transformers were installed for every $1 \mathrm{MW}$ of generator capacity installed.

\subsubsection{Number of Transformers Installed Each Year by Type}

From the MVA of each type of transformer sold and the simplified model of the utility grid, the number of each type of transformer installed in each year is determined. The simplified utility grid, as shown in Figure 7.2, is a construct that was developed to reduce the number of transformers analyzed to a manageable number. As explained in the description of the base cases (Section 3.5.1), there are three different varieties (sizes and applications) of generation step-up transformers, two varieties of autotransformers, and one variety of other transformers (step-down units).

The current mix of generating capacity, as determined from data provided to EIA by utilities on Form EIA-860, is $61.7 \%$ baseload, $25.1 \%$ cycling (or intermediate load), and $13.2 \%$ peaking.

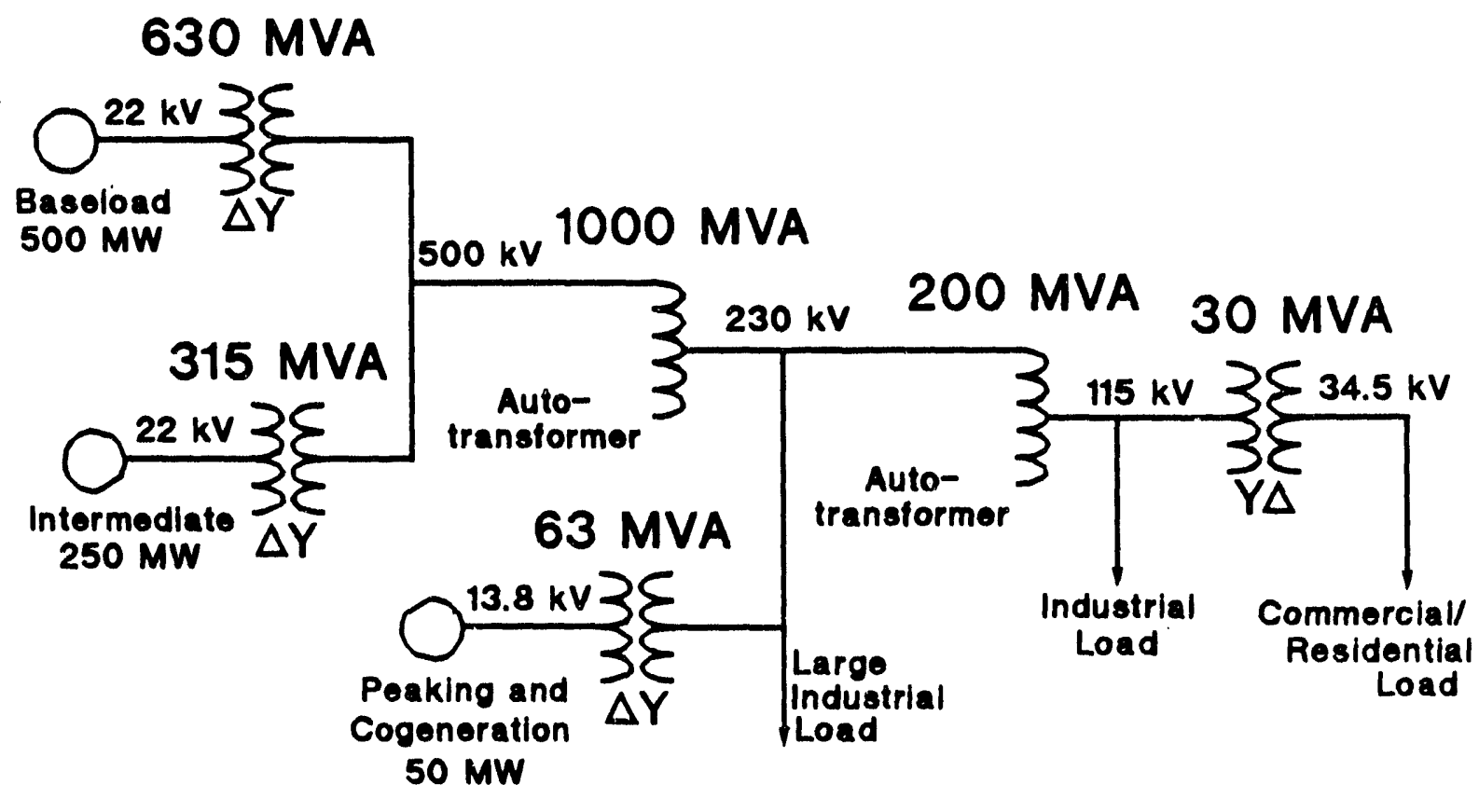

Figure 7.2. Simplified Utility Grid 
Minimal historical data are arailable on the actual percentages of autotransformers at each size and voltage. Thus, for this study the total autotransformer MVA was assumed to be evenly split between the two varieties of autotransformers shown in Figure 7.2. Finally, all "other transformers" were assumed to be step-down transformers.

Therefore, the actual number of transformers installed for any of the six varieties shown in Figure 7.2 can be calculated by multiplying the previously determined MVA for each type (generation step-up, autotransformer, or step-down) by the percentage of the total that the individual variety represents. The MVA of each variety of transformer installed per MW of generator capacity installed is shown in Table 7.2.

\subsubsection{Calculation of Surviving Transformers by Year}

The Weibull distribution is often used in reliability analysis to model natural failures of devices. The three parameters of the Weibull distribution are the location parameter delta $(\delta)$, the shape parameter beta $(\beta)$, and the scale parameter theta $(\theta)$.

The location parameter is simply the minimum possible life of the device. A typical value used in electrical system analysis is zero, and this value was assumed to be valid for transformers. The only effect $\delta$ has on the Weibull distribution is to shift the distribution along the x-axis.

The shape parameter $\beta$ determines the shape of the distribution. For large values of $\beta$, the shape of the distribution resembles that of the normal distribution. A value of 8 was selected for $\beta$; this results in a distribution with a relatively sharp peak. Thus, transformer failures are tightly grouped around the average lifetime ( 30 years was used in this study).

Table 7.2. Transformer Capacity Installed (MVA) per MW of Generator Installation

\begin{tabular}{|c|c|c|c|}
\hline Transformer Type & $\begin{array}{l}\text { Rating, } \\
\text { MVA }\end{array}$ & Application & $\begin{array}{l}\text { Transformer MVA/ } \\
\text { Generator MW }\end{array}$ \\
\hline Generation step-up & 630 & Baseload & 0.777 \\
\hline Generation step-up & 315 & Cycling & 0.316 \\
\hline Generation step-up & 63 & Peaking & 0.166 \\
\hline Autotransformer & 1000 & Transmission & 1.084 \\
\hline Autotransformer & 200 & Subtransmission & 1.084 \\
\hline Step-down & 30 & $\begin{array}{l}\text { Transmission/ } \\
\text { distribution interface }\end{array}$ & 1.072 \\
\hline
\end{tabular}


The scale parameter $\theta$ is linearly related to the average life of the equipment. In addition, $63.2 \%$ of all equipment failures will occur between the minimum life value $\delta$ and the scale parameter value $\theta$. This percentage is valid regardless of the parameter values. A value of 31.9 was selected for the scale parameter, which results in a mean life of 30.0 years and a median life of 30.4 years.

Figure 7.3 shows the annual replacement probability (attributable to failure and retirement) for the transformers as determined by the Weibull distribution. Replacement probabilities provided in Figure 7.3 are based on the initial transformer population (i.e., the number of transformers installed) in a given year. Thus, $2 \%$ of all the transformers installed 22 years ago will be replaced when they are 22 years old. Also, Figure 7.3 shows a vertical line at 30 years because it was assumed that step-up transformers (used at generating stations) will be taken out of service after 30 years when the generating station is replaced. [(EPRI (1986) recommends using 30 years as the lifetime of generating stations.]

Because most transformers were installed during periods of relatively low real energy prices, and because old transformers were less efficient than new transformers, energy losses were valued less in the past. Therefore, transformer lifetimes can be expected to become shorter than their 30- to 50 -year average as the value of losses increases.

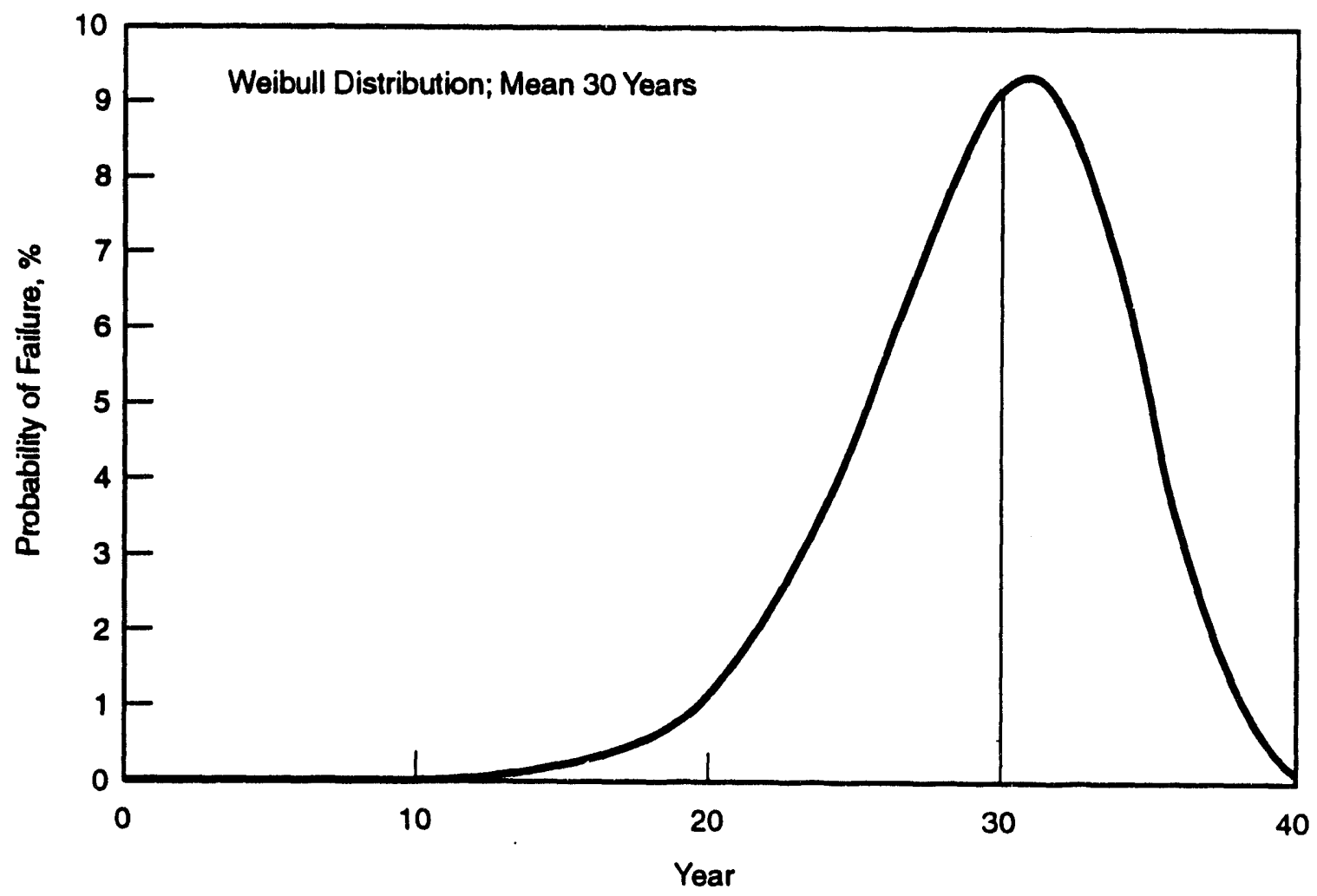

Figure 7.3. Transformer Annual Replacement Probability 
The information on existing generating capacity, transformer installations per GW of generating capacity, and transformer replacement provides the basis for developing an initial inventory of transformers, by size and age, as of 1989. To determine the number of transformers that need to be added to this inventory each year, transformers must be replaced according to the Weibull distribution (2.0 in Figure 7.1) and transformers must te added to account for load growth (3.0 in Figure 7.1).

Information from the North American Reliability Council (NERC 1988) indicates that electrical generating requirements will increase at an average annual rate of $1.9 \%$ through 1997 . For this analysis, this rate of increase in the need for electrical generating capacity was assumed to continue through 2030 . This information was then used along with the information on transformer requirements per GW of generating capacity installed, to determine the number of new transformers required. with time.

Throughout this analysis, it was assumed that the mixture of transformer sizes did not change

\subsection{Utility Market Penetration of HTS}

This section describes the market penetration methodology developed to estimate the size of the utility market for HTS transformers. This methodology assumes that utilities will not replace existing conventional transformers with HTS transformers for purely economic reasons. Instead, the methodology assumes that utilities will delay selecting a new transformer until either 1) an installed transformer has failed or 2) would be economically replaced with a new conventional transformer. When a transformer must be replaced, utilities choose between conventional and HTS transformers based on a comparison of life-cycle costs.

\subsubsection{Overview of the Transformer Selection Process}

The transformer selection methodology is presented schematically in Figure 7.4, which is an expansion of boxes 5.0 and 6.0 from Figure 7.1. The process begins with the need to install transformers each year (box 4.0) for either replacement or load growth purposes. Each year, the utilities will compare conventional and HTS transformer life-cycle costs (LCCs) (5.1). Information on LCCs is supplied by the technical tasks (5.2 and 5.3) and includes capital cost, operating and maintenance costs, and the quantity of energy losses.

For conventional transformers, LCCs are assumed to remain constant, in real terms, over time. On the other hand, HTS capital costs are allowed to vary with time and will most likely be greater than conventional transformer costs, at least in the first years of commercial introduction. HTS capital costs are assumed to decrease with time as production efficiency improves (see Section 5.2.2.3).

The next step is to find energy values for losses that equate the LCCs for HTS and conventional transformers (5.4). Utilities that value their energy at greater than these energy values are assumed to select HTS transformers over conventional transformers (5.5). Adjustments (6.0) are made to annual HTS sales estimates to reflect risk (6.1), regulatory climate (6.2), and utility technical constraints 


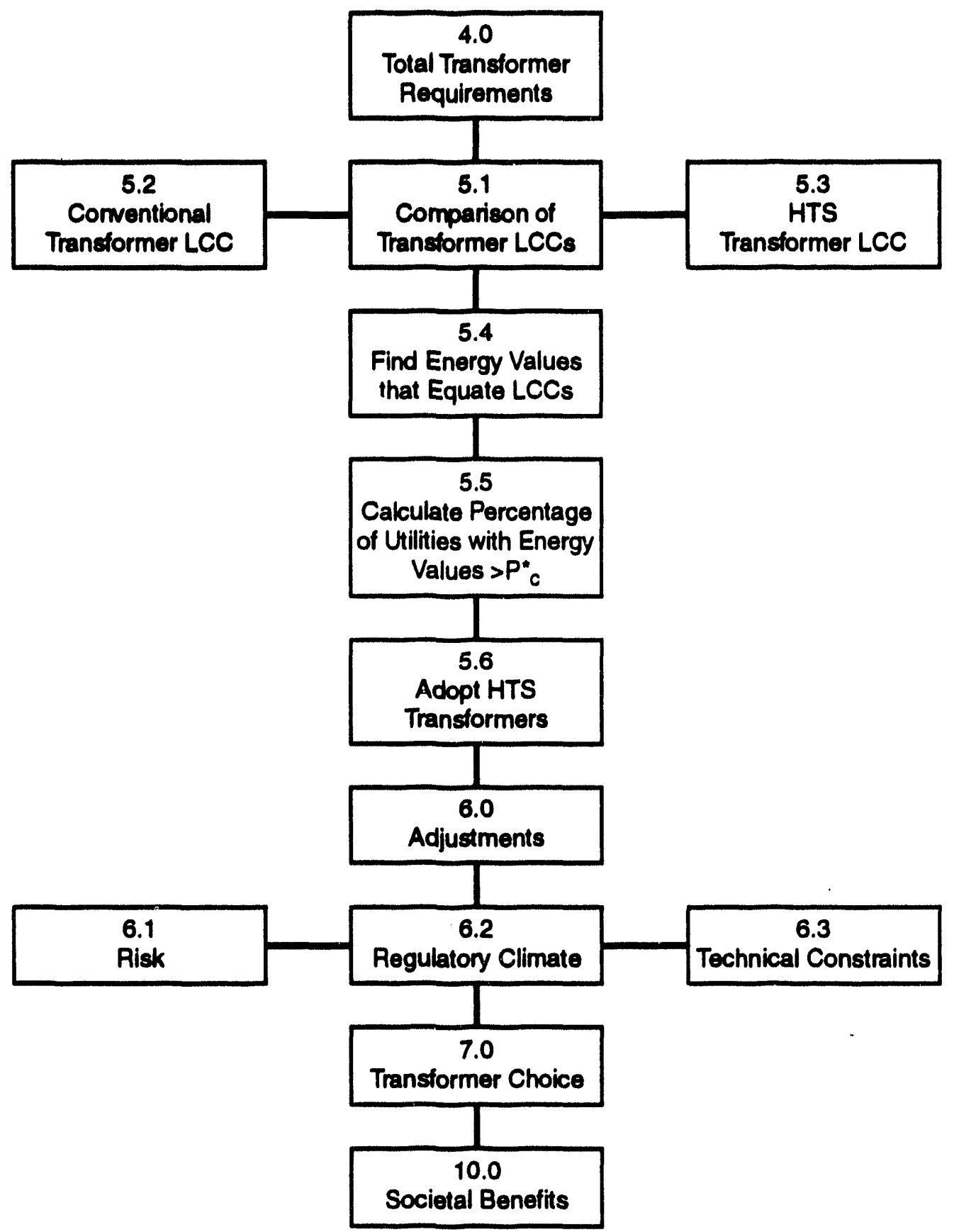

Figure 7.4. Transformer Selection Methodology 
(6.3). Once these adjustments are considered, the selection of transformers is finally made (7.0). Functionally, the selection of either HTS or conventional transformers is

$$
T_{i}=f(L C C, I R, T C, C C)
$$

where $\quad T_{1}=$ the type of transformer selected, either HTS or conventional

$\mathrm{LCC}=$ relative life-cycle costs

IR = initial risk associated with the purchase of a new technology

TC = technical constraint that represents the technical capabilities of a utility

$\mathrm{CC}=$ capital constraints that represents some utilities' concerns over the relatively higher capital costs of HTS transformers.

The following sections provide the methodological details of the transformer selection process used to estimate the potential utility market.

\subsubsection{Transformer Selection Process Details}

The transformer selection process is initiated once a transformer need is identified. The process that utilities would use is based on the following assumptions:

- Utilities require transformers either to meet load growth or to replace failed or retired transformers.

- When a utility needs a transformer, it will choose between conventional and HTS based on relative LCCs. Life-cycle costs, however, are not the only consideration. The utility will also consider its own technical capabilities, the risk associated with selecting a new technology, and the initial capital costs of HTS transformers.

- HTS capital costs will decrease over time.

- Conventional transformers are a mature technology, and their capital costs will remain constant over time.

- Energy prices will remain constant (in real terms) over time.

- Energy values, $\mathrm{P}^{*}$ and $\mathrm{P}^{*}$, will equate the two LCCs in a given year.

- Any utility with an energy value greater than $\mathrm{P}^{*}$. will select an HTS transformer, and those utilities with energy values of less than $P^{*}$ will select conventional transformers.

The transformer population matrix and the process of failure, retirement, and load growth were presented in Section 7.1.

Utilities will choose the type of transformer to be installed based on relative LCCs.

Conventional transformer LCCs are represented by 


$$
L C C_{c}=K_{c}+O \& M_{c}+P Z_{c}\left(P^{*}\right)
$$

where LCC $_{0}=$ life-cycle cost of conventional transformers

$\mathrm{K}_{\mathrm{c}}=$ present value of conventional transformer capital costs

$O \& M_{c}=$ present value of conventional transformer operation and maintenance costs

$\mathrm{PVL}_{\mathrm{c}}=$ present value of conventional transformer energy losses divided by the mean value of losses in $\$ / \mathrm{kWh}$

$\mathbf{P}_{0}^{*}=$ utility energy values, which are based on the proportion of load and no-load losses present in a conventional autotransformer and on the proportion of operating versus nonoperating losses for step-up transformers.

HTS transformer LCCs are represented by

$$
L C C_{s}=K_{s}+O \& M_{s}+P L_{s}\left(P^{*}\right)
$$

where LCC $=$ life-cycle cost of HTS transformers

$\mathrm{K}_{\mathbf{1}}=$ present value of HTS transformer costs

$\mathrm{O \& M}_{1}=$ present value of HTS transformer operation and maintenance costs

$\mathrm{PVL}_{\mathbf{1}}=$ present value of HTS transformer energy losses divided by the mean value of losses in $\$ / \mathrm{kWh}$

$\mathrm{P}^{*}:=$ utility energy values, which are based on the proportion of load and no-load losses present in HTS autotransformers and life-cycle costs of the HTS transformer are that less than those of the conventional transformer. That is, if

$$
L C C_{c}>L C C_{s}
$$

Or, combining Equations (7.2) and (7.3),

$$
K_{c}+O \& M_{c}+P Z_{c}\left(P^{*}\right)>K_{s}+O \& M_{s}+P V L_{s}\left(P{ }^{*}\right)
$$

Life-cycle costs are compared for each year of the analysis.

The energy prices are also related:

$$
P_{c}=f\left(P_{\jmath}\right)
$$

\subsubsection{Valuing Energy Losses}

The value that utilities place on transformer energy losses is a function of 1) their costs of generating and transmitting electricity, 2) the combination of transformer load and no-load losses (for autotransformers) or operating and nonoperating losses (for step-up transformers). Commercial or 
industrial energy prices charged by the utilities are not (by themselves) sufficient for valuing these energy losses (see Section 5.2.4). Consequently, we constructed new energy price distributions that would properly represent the value to the utility of these energy losses. The procedure used to develop these new energy value distributions is described in the following subsections.

\subsubsection{Autotransformers}

Loss characteristics vary according to transformer type (i.e., autotransformers versus step-up transformers), as well as between HTS and conventional transformers. Conventional autotransformers have both load and no-load energy losses; HTS autotransformers have only no-load losses.

Because no-load losses in an autotransformer are a baseload demand (i.e., constant demand whenever the transformer is energized), utilities will value transformer no-load losses as the cost of generating electricity at baseload plants plus the cost of transmission losses. Load losses vary with the square of the load and are most significant when the grid is operating near its peak capacity (peaking plants are operating); thus, load losses will be valued by utilities at the cost of generating electricity from peaking plants plus the cost of transmission losses.

Because conventional autotransformers have both load and no-load losses, we had to construct a distribution from available energy price information to properly value these losses. The distribution constructed for autotransformers was based on the distribution of industrial energy prices (IEP) (Energy User News 1989) for 160 major utilities in the United States. The mean and standard deviation of any new energy price distributions that were constructed for valuing load and no-load losses were assumed to have the same ratio of mean to standard deviation as the IEP; that is,

$$
\frac{\mu_{\text {lep }}}{\sigma_{\text {lep }}}=\frac{\mu_{\text {lood }}}{\sigma_{\text {load }}}=\frac{\mu_{\text {no-load }}}{\sigma_{\text {no-lood }}}
$$

After we constructed the individual distributions for load and no-load losses, we constructed combined distributions reflecting the proportion of load and no-load losses present in a specific type of transformer. The mean for the combined distribution $\left(\mu_{\mathrm{com}}\right)$ is a function of the proportion of load to no-load losses present in a given autotransformer; that is,

$$
\begin{gathered}
\mu_{\text {com }}=\left(\mu_{\text {load }}\right)[\text { load losses as percentage of total losses }]+\left(\mu_{\text {no-load }}\right) \\
{[1-\text { load losses as percentage of total losses }]}
\end{gathered}
$$

Because we assumed that the ratio of the mean to standard deviation remains the same for all electricity price distributions, we calculated the appropriate standard deviation for the combined distribution. A combined distribution was constructed for both conventional and HTS autotransformers. Because HTS transformers were assumed not to have load losses, the combined distribution for the HTS autotransformer is identical to the no-load loss distribution. 
Energy prices are assumed to remain constant, in real terms, over time. This assumption agrees with the forecasts of energy prices estimated by the EIA (1989). If energy prices were allowed to vary (in real terms) over time, the transformer type selected by utilities would vary only slightly.

\subsubsection{Step-Up Transformers}

As noted above, the loss characteristics of step-up transformers differ from those of autotransformers. HTS step-up transformers suffer from "nonoperating" losses. These losses are assumed to be present because it is believed that HTS step-up transformers will be kept at operating temperatures even when they are not energized. However, conventional step-up transformers do not require cooling when the transformer is not energized, so these transformers do not suffer from nonoperating losses.

The process for constructing price distributions for HTS step-up transformers is similar to that used to construct the price distributions for conventional and HTS autotransformers. First, we constructed a price distribution for nonoperating losses, assuming that the ratio of the mean to standard deviation is equal to that of the IEPS. Next, we determined the percentage of operating versus nonoperating losses present in each step-up transformer design. Finally, we combined the nonoperating and operating losses to develop a combined distribution.

\subsubsection{Selecting HTS Versus Conventional Transformers}

The process for determining which utilities will select HTS transformers rather than conventional transformers is to find appropriate energy loss values, $\mathrm{P}^{*}$, and $\mathrm{P}^{*}$, such that the following equation is satisfied:

$$
K_{c}+O \& M_{c}+P V L_{c}\left(P^{*}\right)=K_{s}+O \& M_{s}+P V L_{s}\left(P^{*}{ }_{s}\right)
$$

Because utilities will select the technology that offers the lower LCC [Equations (7.4) and (7.5)], it follows that any utility that values energy losses at greater than $\mathrm{P}^{*}{ }_{\mathrm{c}}$ and $\mathrm{P}^{*}{ }_{\mathrm{g}}$ will select HTS transformers over conventional transformers. Because utilities value energy losses relatively (to all other utilities) the same, the percentage of utilities that value energy losses greater than $P_{c}^{*}$ will be the same as those that value energy losses at greater than $\mathrm{P}^{*}$. This is comparable to equating the $z$-statistic for the 2 normalized $(0,1)$ distributions. That is,

$$
Z_{c}=Z_{s}
$$

or

$$
Z_{c}=\frac{P_{c}^{*}-\mu_{c}}{\sigma_{c}}
$$


and

$$
Z_{s}=\frac{P_{s}^{*}-\mu_{s}}{\sigma_{s}}
$$

If Equations (7.11) and (7.12) are set equal to each other, then

$$
\frac{P_{c}^{*}-\mu_{c}}{\sigma_{c}}=\frac{P^{*}-\mu_{c}}{\sigma_{s}}
$$

Solving for $\mathrm{P}^{*}$,

$$
P_{s}^{*}=\sigma_{s} \frac{\left(P_{c}-\mu_{c}\right)}{\sigma_{c}}+\mu_{s}
$$

Substituting Equation (7.14) into Equation (7.9) and solving for $\mathrm{P}^{*}$,

$$
P_{c}^{*}=\frac{\sigma c\left[K_{s}-K_{c}+O \& M_{s}-O \& M_{c}+P V L_{s} \mu_{s}\right]-P V L_{s} \sigma_{s} \mu_{c}}{\sigma_{c} P V L_{c}-P V L_{s} \sigma_{s}}
$$

where $\quad \sigma c=$ standard deviation of the combined load/no-load energy prices for conventional transformers

$K_{1}=$ present value of capital cost of HTS transformers

$\mathrm{K}_{\mathrm{o}}=$ present value of capital cost of conventional transformers

O\&M $=$ present value of operating and maintenance cost for HTS transformers

$O \& M_{0}=$ present value of operating and maintenance cost for conventional transformers

$\mathrm{PVL}_{\mathbf{s}}=$ present value of HTS transformer energy losses divided by the mean value of losses in $\$ / \mathbf{k W h}$

$\mu \mathrm{S}=$ mean energy price for the energy loss distribution for HTS transformers

$\sigma \mathrm{S}=$ standard deviation of the energy price distribution for HTS transformers

$\mu_{0}=$ mean energy price for the energy loss distribution for conventional transformers

$\mathrm{PVL}_{\mathrm{c}}=$ present value of conventional transformer energy losses divided by the mean value of losses in $\$ / \mathbf{k W h}$.

The process for determining the number of HTS transformers installed in a given year is to calculate $\mathrm{P}^{*}$ and assume that any utility with a combined energy value greater than this value will adopt HTS. The percentage of utilities with energy values higher than $\mathrm{P}^{*}$ is multiplied by the total number of transformers of each size needed in that year. Summing these values over time will yield the cumulative market penetration curve. 


\subsubsection{Adjustments}

Several factors will affect the actual number of HTS transformers that will be sold in a given year. For this analysis, these factors have been categorized as either initial risk, technical constraints, or capital constraints. These are explained in the following paragraphs.

\subsubsection{Initial Risk}

The adjustment for initial risk is the result of risk-averse behavior on the part of some utilities in adopting HTS transformers. Concerns regarding initial risk translate into delays in buying these transformers until a utility-sponsored testing program is completed. Information for this adjustment was compiled through a telephone survey of utility representatives. The function used to represent this adjustment is

$$
\text { Initial risk }=0.103145+0.366855(\ln (\text { year }))
$$

where year $=$ the number of years after the technology becomes commercially available (the first year it is available is year 1 )

\subsubsection{Technical Constraint}

The technical constraint adjustment represents the fact that some utilities will not adopt HTS transformers because such installations require a technical sophistication that they do not have. Utilities with these technical constraints are assumed to represent about $2 \%$ of the potential HTS sales each year. This adjustment is a constant each year and is measured as the percentage of utilities that have the necessary technical capabilities to install and maintain the more sophisticated HTS transformers. This assumption correlates well with the information gathered in our survey of utilities.

$$
\text { Technical constraint }=0.98
$$

\subsubsection{Capital Costs}

The adjustment for capital costs represents the fact that some utilities are concerned with initial capital costs (often due to regulatory concerns) as well as with life-cycle costs. Again, this information was gleaned from our survey of utility representatives. The adjustment is based on the relative capitai costs of HTS and conventional transformers and is represented by

$$
\text { Capital cost }=0.99526\left(K / K_{c}\right)^{-1.3666}
$$

where $K_{3}=$ the present value of HTS transformer costs

$\mathrm{K}_{\mathrm{c}}=$ the present value of conventional transformer costs. 


\subsubsection{Total Adjustment}

The total adjustment made to the HTS transformer annual sales is the product of the three adjustments; that is,

$$
\begin{aligned}
\text { Total adjustments }= & {[(0.103145+0.366855(\ln (\text { year }))][0.98]} \\
& {\left[0.99526\left(K_{J} / K_{C}\right)^{-1.366}\right] }
\end{aligned}
$$

The degree of precisi on of the above equations does not reflect the quality of the data, but rather the resolution of the curve fits to the survey data.

\subsection{Benefits to the Nation}

The methodology used to estimate the benefits to the nation from adopting a relatively less expensive and more efficient transformer is detailed in this section. The benefits to the nation from installing HTS transformers include 1) the actual energy savings from adopting a more energyefficient transformer and 2) the dollar savings from adopting a relatively lower cost and more energy-efficient transformer.

Calculating the actual energy savings from installing HTS transformers is fairly straightforward. The savings are merely the difference in the energy losses of conventional and HTS transformers multiplied by the number of surviving HTS transformers in a given year; that is,

$$
\text { (Energy savings)i }=\left(L_{c}-L_{s}\right) * \text { (Number of surviving HTS transformers in year i) }
$$

where $\quad L_{c}=$ annual energy losses from conventional transformers

$L_{s}=$ annual energy losses from HTS transformers.

Calculating the value of installing a relatively less expensive and more efficient transformer involves evaluating the difference in capital, O\&M, and energy losses over time. The capital costs of the HTS transformers are assumed to decrease over time. Consequently, the difference in the present value of the capital costs must be evaluated each year that HTS transformers are installed; that is,

$$
\left.\left(K_{c}-K_{s}\right)_{i} * \text { (Number of HTS transformers installed in year } i\right)
$$

The benefits/costs of installing a transformer that requires less/more maintenance are the reduced/increased dollar expenditures for O\&M on the HTS transformers that are operating in a given year; that is,

$$
\left.\left(O \& M_{c}-O \& M_{9}\right)_{i} * \text { (Number of surviving HTS transformers in year } i\right)
$$


In addition to the difference in capital and O\&M costs, reductions in energy losses from installing HTS transformers also reduce the need to generate electricity. Functionally, this is

$$
\text { Value of energy lasses }=\bar{P}_{c}\left(L_{c}\right)-\bar{P}_{2}\left(L_{2}\right)
$$

where $\quad \overline{\mathbf{P}}_{0}=$ the average of the energy values greater than $\mathrm{P}_{0}$

$\mathbf{P}_{i}$. = the average of the energy values greater than $\mathbf{P}^{\circ}$.

Estimating the value of reduced energy losses to the nation is not as straightforward as is estimating the capital and O\&M savings because energy loss values must be calculated that are the average of the energy values greater than $P_{c}^{*}$ and $P_{a}^{*}$. The values, $\bar{P}_{c}$ and $\bar{P}_{a}$, are calculated by finding the expected values of the tails of the two energy value distributions presented in Figure 7.5.

Calculating the expected values for these two distributions requires evaluating the following integral for both the $\mathrm{P}^{*}$ and the $\mathrm{P}^{*}$, distributions as follows:

$$
\bar{P}_{s, c}=\int_{p_{*}^{*}}^{\infty} \frac{1}{\sigma \sqrt{2} \pi} P^{*} e-\left[0.5 \frac{\left(P^{*}-\hat{P}^{2}\right.}{\sigma^{2}}\right] d p
$$

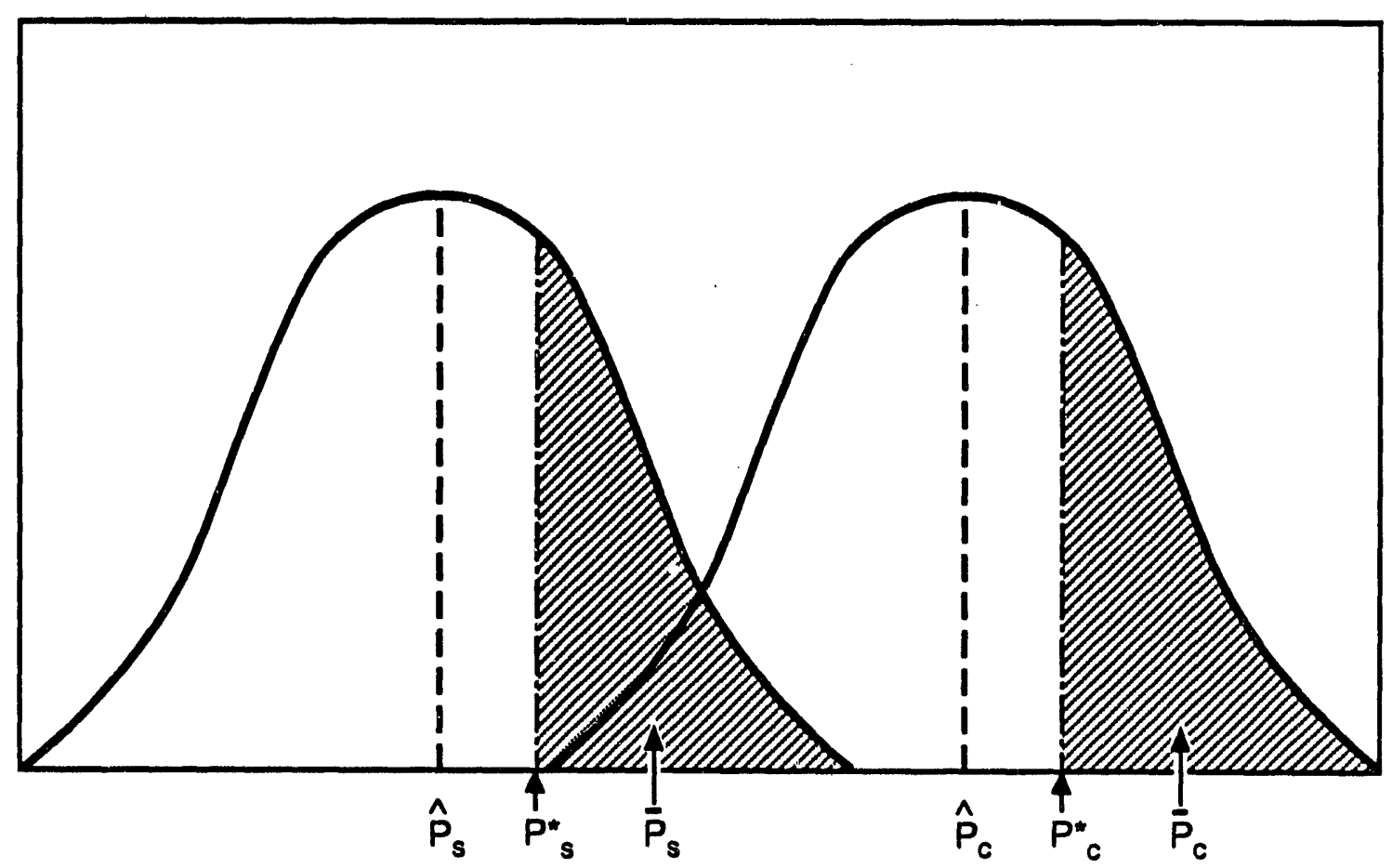

Figure 7.5. The Averages of Energy Values Greater than $P^{*}$, 
The values obtained from evaluating these integrals, $\overline{\mathrm{P}}_{\mathrm{f}}$ and $\overline{\mathrm{P}}_{\mathrm{c}}$, are multiplied by the corresponding annual energy losses, $\mathrm{L}_{\mathrm{c}}$ and $\mathrm{L}_{\mathrm{c}}$. These values are then discounted and are summed over time to estimate the value of the energy savings.

The benefits of installing HTS transformers will occur over time. Thus, it is necessary to discount the expected future cash flows to arrive at the present value. The appropriate discount rate to use for discounting depends on who is receiving the benefits of the new technology.

If utilities choose to install HTS transformers, they will be installing transformers with relatively lower capital costs (after learning-curve effects) and with relatively higher operating efficiencies. Because only capital costs are included in the utility's rate base, and the benefit of reduced operating costs are immediately passed on to consumers, the benefits from installing HTS transformers will be to the consumers in the form of lower electricity rates. In addition, these benefits will accrue to the consumers at minimal risk. Thus, the appropriate discount rate must reflect the fact that these benefits will be accruing both to consumers and at no risk.

For this analysis, the borrowing rate for the government was selected as the appropriate discount rate. Over the period 1926 through 1987 inclusive, the arithmetic mean real rate of return on long-term government bonds has been 1.7\% (Ibbotson 1988). This average was used as the discount rate for discounting HTS benefits.

The total value of the benefits to the nation of adopting HTS transformers is the sum of the discounted values from Equations (7.21), (7.22), and (7.23). All benefits are discounted back to the year 1989.

\subsection{Market Penetration Evaluation of HTS Transformer Designs}

The market penetration methodology was used to evaluate the utility market potential and benefits to the nation of four HTS transformer types and six transformer sizes. Benefits to the nation from adopting HTS transformers are presented in this section, as well as market share information.

Two market penetration scenarios were used to evaluate the market potential of HTS transformer designs: a base case and a sensitivity case. For the base case, the initial year of sales considered was assumed to be 2000 and the final year of market penetration was assumed to be 2020 . Because the benefits of adopting HTS transformers extend beyond the final year of penetration, we continued to measure benefits to the year 2060. For the sensitivity case, the initial year of market penetration remained 2000, but the final year of sales considered was extended to 2030 and the measurement of benef ${ }_{\imath}$ s continued until 2070.

\subsubsection{Dollar Savings from Adopting HTS Transformers}

Information on the dollar benefits of adopting HTS transformers is presented first in this section to illustrate the relative magnitude of savings from various sized transformers as well as the relative savings from the different transformer designs. 
The base case results indicate the following:

- The largest dollar benefit from adopting HTS transformers is achieved by the cryostable conventional core design, with the present value of benefits being in excess of $\$ 17$ billion.

- The smallest dollar benefit is from the ultrastable amorphous core transformers, with a total present value of benefits of $\$ 14.5$ billion.

The dollar benefits to the nation of adopting cryostable transformers exceed the benefits of adopting ultrastable transformers for almost all sizes (Table 7.3). This fact is primarily the result of the more rapid decrease in cryostable transformer capital costs as reflected in the capital cost-adjustment factors (see Section 5.2.2.3).

For almost all sizes, conventional core transformers yield greater dollar benefits than do their amorphous core counterparts. The primary reason for this result is that amorphous core transformers cost more than conventional core transformers.

Extending both the final year of sales considered and the final year for measuring dollar benefits by 10 years increases the dollar benefits of adopting HTS transformers. The dollar benefits of adopting the cryostable conventional transformers increase from $\$ 17$ billion to more than $\$ 28$ billion (Table 7.4). Similar increases in dollar benefits are demonstrated by all HTS transformers in the sensitivity case; however, relative dollar benefits do not change.

The contribution of capital, O\&M, and energy savings to the overall dollar benefits from adopting the 1000-MVA cryostable conventional transformer is illustrated in Figure 7.6. From the initial year of introduction through the final year of market penetration, both the capital and energy savings contribute equally to the overall savings. The significant contribution of energy savings to total savings results from accounting for energy savings in every year during which the HTS transformer is still in service. However, after 2020 (the final year of sales considered), the energy savings represents the major dollar benefit of adopting the cryostable transformer. The contribution of capital cost savings is relatively lower because these savings are accounted for only in the year of introduction. Operating and maintenance cost savings contribute little to the overall savings because O\&M costs for conventional transformers are relatively minor.

\subsubsection{Energy Savings from Adopting HTS Transformers}

The benefits of adopting HTS transformers include not only the dollar savings in capital, operating and maintenance, and energy costs, but also the reduced amount of energy that must be generated in the future (all other factors held constant). Table 7.5 presents a summary of the amount of energy saved (as measured in billions of kilowatt-hours) by adopting each of the four HTS transformer types. Only base case results are presented. 
Table 7.3. Dollar Benefits from Adopting HTS Transformers, Base Case (in 1989 \$)

\begin{tabular}{|c|c|c|c|c|}
\hline & \multicolumn{2}{|c|}{ Cryostable } & \multicolumn{2}{|c|}{ Ultrastable } \\
\hline & Amorphous & Conventional & Amorohous & Conventional \\
\hline \multicolumn{5}{|l|}{ Base case (years) } \\
\hline Initial market penetration & 2000 & 2000 & 2000 & 2000 \\
\hline $\begin{array}{l}\text { Final year of market } \\
\text { penetration }\end{array}$ & 2020 & 2020 & 2020 & 2020 \\
\hline Analysis end & 2060 & 2060 & 2060 & 2060 \\
\hline \multicolumn{5}{|l|}{ Dollar savings (billions) } \\
\hline 30-MVA step-down & 6.6 & 7.5 & 7.0 & 7.4 \\
\hline 200-MVA autotransformer & 2.8 & 3.7 & 2.7 & 3.3 \\
\hline 1000-MVA autotransformer & 2.1 & 2.6 & 1.9 & 2.3 \\
\hline 63-MVA step-up (peaking) & 0.4 & 0.3 & 0.3 & 0.3 \\
\hline 315-MVA step-up (cycling) & 0.8 & 0.8 & 0.7 & 0.6 \\
\hline 630-MVA step-up (baseload) & 2.3 & 2.4 & 2.0 & 2.1 \\
\hline $\operatorname{Total}^{(a)}$ & 15.0 & 17.2 & 14.5 & 16.0 \\
\hline
\end{tabular}

(a) Totals may not sum because of rounded numbers.

Table 7.4. Dollar Benefits from Adopting HTS Transformers, Sensitivity Case

\begin{tabular}{|c|c|c|c|c|}
\hline & \multicolumn{2}{|c|}{ Cryostable } & \multicolumn{2}{|c|}{ Ultrastable } \\
\hline & Amorphous & Conventional & Amorphous & Conventional \\
\hline \multicolumn{5}{|l|}{ Sensitivity case (years) } \\
\hline Initial market penetration & 2000 & 2000 & 2000 & 2000 \\
\hline $\begin{array}{l}\text { Final year of market } \\
\text { penetration }\end{array}$ & 2030 & 2030 & 2030 & 2030 \\
\hline Analysis end & 2070 & 2070 & 2070 & 2070 \\
\hline \multicolumn{5}{|l|}{ Dollar savings (billions) } \\
\hline 30-MVA step-down & 10.8 & 12.3 & 12.0 & 12.8 \\
\hline 200-MVA autotransformer & 4.6 & 6.0 & 4.6 & 5.7 \\
\hline 1000-MVA autotransformer & 3.4 & 4.2 & 3.2 & 3.9 \\
\hline 63-MVA step-up (peaking) & 0.7 & 0.6 & 0.6 & 0.5 \\
\hline 315-MVA step-up (cycling) & 1.4 & 1.3 & 1.2 & 1.2 \\
\hline 630-MVA step-up (baseload) & 3.9 & 4.1 & 3.7 & 3.9 \\
\hline Total $^{(a)}$ & 24.9 & 28.4 & 25.3 & 27.8 \\
\hline
\end{tabular}

(a) Totals may not sum because of rounded numbers. 


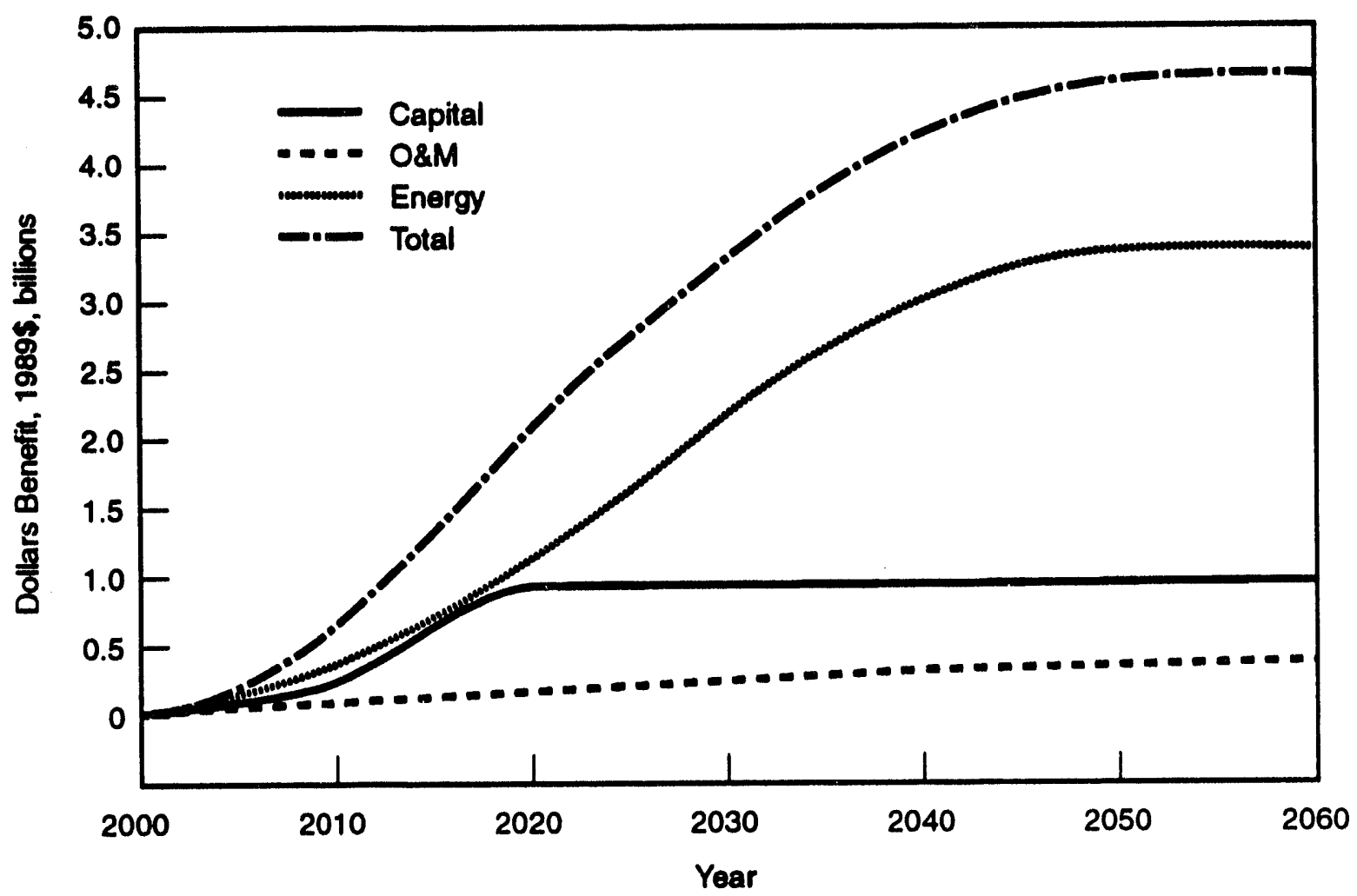

Figure 7.6. Dollar Benefits from Adopting HTS

Table 7.5. Energy Savings from HTS Transformers (billions $\mathrm{kWh}$ )

\begin{tabular}{|c|c|c|c|c|c|c|c|}
\hline & \multicolumn{3}{|c|}{ Autorranformen } & \multicolumn{4}{|c|}{ Step-Up Transformers } \\
\hline & 30MVA & $200 \mathrm{MVA}$ & $1000 \mathrm{MVA}$ & 63 MVA & 315 MVA & 630 MVA & Total \\
\hline $\begin{array}{l}\text { Cryostable } \\
\text { Amorphous Core }\end{array}$ & 7.7344 & 8.8886 & 9.0088 & 1.1944 & 16.514 & 45.1001 & 88.4409 \\
\hline $\begin{array}{l}\text { Cryoutable } \\
\text { Conventional Core }\end{array}$ & 86.2235 & 40.8761 & 28.0543 & 1.1405 & 19.1908 & 57.0675 & 232.5528 \\
\hline $\begin{array}{l}\text { Ultrastable } \\
\text { Amorphous Core }\end{array}$ & 75.5402 & 29.0165 & 19.7207 & 1.6839 & 17.7348 & 51.2966 & 194.9928 \\
\hline $\begin{array}{l}\text { Ultrastable } \\
\text { Conventional Core }\end{array}$ & 133.7467 & 53.1723 & 37.0594 & 1.3873 & 19.0956 & 64.1586 & 308.6204 \\
\hline
\end{tabular}

The amount of energy saved from adopting the ultrastable transformers is significantly larger than energy saved from adopting the cryostable designs. This result is because of the relatively higher operating efficiencies demonstrated by the ultrastable designs, a factor that could increase the 
market attractiveness of ultrastable transformers, particularly if energy costs increase (in real terms) over time [they were assumed to remain constant in real terms for this analysis (EIA 1989)].

In almost all cases, the energy savings from adopting amorphous core transformers is less than the energy savings from adopting conventional core transformers. This is because conventional transformers with amorphous cores typically have higher operating efficiencies. Thus, because the comparisons are made between the HTS transformer and its conventional counterpart, the energy savings from adopting the HTS amorphous core design is generally less than it is for other core designs.

The energy savings from adopting HTS transformers has been understated in this analysis because transformers of less than 30 MVA were not considered. If reliable HTS transformers of sizes less than 30 MVA were developed and adopted by the utility industry, the energy savings would be significantly larger. It will take time to design reliable transformers and to convince the utility industry of their reliability.

\subsubsection{Market Penetration Rate of HTS Transformers}

The rate at which the utility industry adopts HTS transformers is a function of several factors: relative life-cycle costs, initial capital costs, utility risk-averseness, and the utility's technical capabilities. With the exception of the technical constraint, the influence of these factors on the adoption of HTS transformers varies with time. Utilities will become more comfortable with adopting HTS transformers as initial capital cost decreases and as reliability is established.

This effect is illustrated by information contained in Table 7.6, which presents the value of the three time-related adjustment factors. These factors are measured as the portion of the potential transformer market captured by HTS transformers. The "potential transformer market" is the share of the market that would be captured by HTS transformers if choices were based exclusively on life-cycle cost comparisons.

The impact of both the high initial capital costs and the perceived risk associated with the new technology is apparent in the early years of market introduction. Of the portion of the market where 1000-MVA conventional core cryostable transformers are the most life-cycle cost effective, only about $4.7 \%$ is captured. This represents about $2.5 \%$ of the total market (conventional and superconducting).

HTS capital costs are expected to decrease over time as production techniques improve. The capital cost adjustment factor (measured as the ratio of conventional transformer capital costs over HTS transformer capital cost--with a limit of 1) increases from 0.4601 in the year 2000 to 1.0000 by the year 2002 for the 1000-MVA conventional core cryostable design. However, the utilities' riskaverseness to adopting a new technology continues to be a limiting factor in the actual amount of the transformer market captured by HTS until the year 2011. After this point, the limited technical capabilities of some utilities prevent HTS from capturing the total potential transformer market.

Figure 7.7 illustrates the cumulative market share captured by the cryostable conventional core 1000-MVA transformer and the ultrastable conventional core 30-MVA transformers (independently) 
Table 7.6. Value of Market Penetration Adjustment Factors for 1000-MVA Conventional Core Cryostable Transformers

$\begin{array}{ccccc}\text { Year } & \begin{array}{c}\text { Technical } \\ \text { Constraints }\end{array} & \begin{array}{c}\text { Capital } \\ \text { Costs }\end{array} & \begin{array}{c}\text { Initial } \\ \text { Risk }\end{array} & \begin{array}{c}\text { Total } \\ \text { Constraint }\end{array} \\ 2000 & 0.9800 & 0.4601 & 0.1031 & 0.0465 \\ 2001 & 0.9800 & 0.7421 & 0.3574 & 0.2599 \\ 2002 & 0.9800 & 1.0000 & 0.5062 & 0.4961 \\ 2003 & 0.9800 & 1.0000 & 0.6117 & 0.5990 \\ 2004 & 0.9800 & 1.0000 & 0.6936 & 0.6797 \\ 2005 & 0.9800 & 1.0000 & 0.7605 & 0.7453 \\ 2006 & 0.9800 & 1.0000 & 0.8170 & 0.8007 \\ 2007 & 0.9800 & 1.0000 & 0.8660 & 0.8487 \\ 2008 & 0.9800 & 1.0000 & 0.9092 & 0.8910 \\ 2009 & 0.9800 & 1.0000 & 0.9479 & 0.9289 \\ 2010 & 0.9800 & 1.0000 & 0.9828 & 0.9631 \\ 2011 & 0.9800 & 1.0000 & 1.0000 & 0.9800\end{array}$

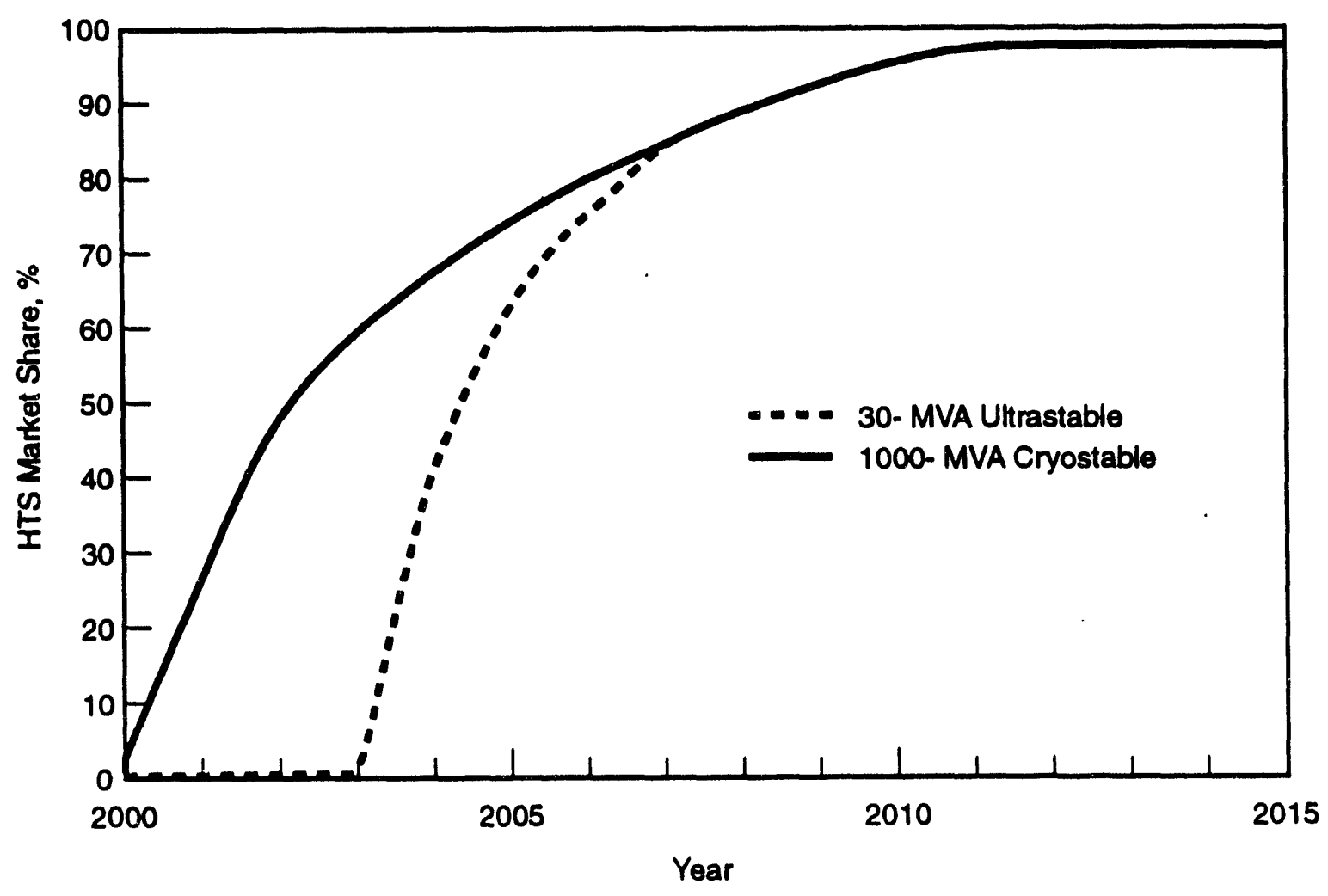

Figure 7.7. Market Share by Year 
between 2000 and 2015. The cryostable conventional core transformer is adopted by the utility market 3 years before the ultrastable design because the cryostable capital costs are relatively less than those of the ultrastable. Risk-averseness also influences the amount of the potential transformer market that HTS transformers represent and continues to affect the adoption of HTS until 2011. Technical constraints, faced by about $2 \%$ of utilities, prevent both HTS transformer designs from capturing the entire transformer market.

The results of the market penetration evaluation indicate that to accelerate the adoption of HTS transformers, efforts should be focused on reducing initial high capital costs of HTS transformers and on developing an industry-sponsored demonstration program. Reduction of these costs and risk concerns will help reduce the time before HTS transformers are introduced. 


\subsection{References}

Argonne National Laboratory. 1988. Advances in Applied Superconductivity: A Preliminary Evaluation of Goals and Impacts. ANL/CNSV-64, Argonne National Laboratory, Argonne, Illinois.

Bonneville Power Administration (BPA). 1988. Superconductivity Applications on an Electric Power System: A System Engineering Study. Bonneville Power Administration, Portland, Oregon.

Borcherds, P. H. 1966. "Physical Limitations on the Size of Superconducting Power Transformers." Proc. IEE 133(12):1953-1956.

Brown, D. R., and G. E. Spanner. 1988. Current Cost and Performance Requirements for Residential Cool Storage System. PNL-6647, Pacific Northwest Laboratory, Richland, Washington.

Brown, D. R., J. A. Dirks, M. K. Drost, G. E. Spanner, and T. A. Williams. 1987. An Assessment Methodology for Thermal Energy Storage Evaluations. PNL-6372, Pacific Northwest Laboratory, Richland, Washington.

California Energy Commission. 1985. The 1985 California Electricity Report-Affordable Electricity in an Uncertain World. California Energy Commission, Sacramento, California.

Carr, W. J., Jr. 1977. "Longitudinal and Transverse Field Losses in Multifilament Superconductors." Institute of Electrical and Electronics Engineers, Inc. (IEEE) IEEE Transactions on Magnetics MAG-13(l):192-197.

Collings, E. W. 1986. Applied Superconductivity, Metallurgy, and Physics of Titanium Alloys. Volume 2: Applications. Plenum Press, New York.

Data Resources, Inc. (DRI). 1987. Energy Review. Lexington, Massachusetts.

Dodge, B. F. 1944. Chemical Engineering Thermodynamics. Chemical Engineering Series, McGraw-Hill Book Company Inc., New York and London.

Electric Power Research Institute (EPRI). 1989. "The Future of Transmission - Switching to Silicon." EPRI Journal, pp. 5-13, Menlo Park, California.

Electric Power Research Institute (EPRI). 1986. Technical Assessment Guide. Palo Alto, California.

Electric Power Research Institute (EPRI). 1987. "Transformers with Lower Losses." EPRI Journal, pp. 23-27, Menlo Park, California.

Electric Power Research Institute (EPRI). 1986. Volume 1: Electrical Supply - 1986. EPRI P-4463-SR, Palo Alto, California. 
Energy Information Administration (EIA). 1989. Annual Energy Outlook: Long-Term Projections. DOE/EIA-0383(89), U.S. Department of Energy, Washington, D.C.

Energy Information Administration (EIA). 1989. Annual Energy Review 1988. DOE/EIA-0384(88), U.S. Department of Energy, Washington, D.C.

Energy User News. 1989. "Ranking of Electricity Prices: Industrial."

Fehur, J. S., K. A. Miller, A. M. Reza, 0. S. Yu, and R. W. Zeren. 1988. Electric Outlook: The Foundation for EPRI R\&D Planning. PED.100.2.88, Electric Power Research Institute, Palo Alto, California.

Funaki, K., Iwakuma, M. Takeo and K. Yamafugi. 1988. "Preliminary Test and Quench Analysis of a 72-kVA Superconducting Four-Winding Power Transformer." Proceedings of ICEC 12, Southampton, England.

Gas Research Institute (GRI). 1987. 1987 GRI Baseline Projection of U.S. Energy Supply and Demand to 2010. Chicago, Illinois.

Guthrie, K. M. 1974. Process Plant Estimating, Evaluation and Control. Craftsman Book Company of America, Solana Beach, California.

Happel, J. and D. G. Jordan. 1975. Chemical Process Economics. 2nd ed. Marcel Dekker, Inc. New York.

Harrowell, R. V. 1970. "Feasibility of a Power Transformer with Superconducting Windings." Proc. IEE 117(1):131-140.

Ibbotson Associates. 1988. Stocks, Bonds, Bills and Inflation: 1988 Yearbook. Ibbotson Associates, Chicago.

Liquid Air Corporation. 1987. "Liquid Air, Industrial Gases and the Ideas That Make Them Work for You." Industrial Gases Division, Oakland, California.

Lorch, H. O. 1969. "The Feasibility of Superconducting Power Transformers." Cryogenics 9:254-361.

Lyon, D. H. 1968. "Pool Boiling of Cryogenic Liquids." In Advances in Cryogenic Heat Transfer, ed. K. J. Bell, AIChE Symposium 87, 64:82-92.

Magnetics Division. 1987. Design Manual Featuring Tape Wound Cores: Catalog TWC-300T. Spang \& Company, Butler, Pennsylvania.

Magnetics Division. 1989. Nickel-Iron Supermendur and Metglas: Catalog MCC-1OOT. Spang \& Company, Butler, Pennsylvania. 
Malozemoff, A. P., W. J. Gallagher, and R. E. Schwall. 1987. "Applications of High-Temperature Superconductivity." In Chemistry of High Temperature Superconductors, pp. 280-306. American Chemical Society, New York.

McFee, R. 1961. "Superconducting Power Transformers - A Feasibility Study." Electrical Engineering 80:754-760.

National Electrical Manufacturers Association (NEMA). 1986. Tenth Biennial Survey of Power Equipment Requirements of the U.S. Electric Utility Industry 1985-1994. Data compiled by Price Waterhouse for the Power Equipment Division of the National Electrical Manufacturers Association, New York.

National Electrical Manufacturers Association (NEMA). 1974. Fourth Biennial Survey of Power Equipment Requirements of the U.S. Electric Utility Industry 1973-1982. Data compiled by the Conference Board for the Power Equipment Division of the National Electrical Manufacturers Association, New York.

North American Electric Reliability Council (NERC). 1988. "Electricity Supply and Demand for 1988-1997." In Annual Data Summary. Princeton, New Jersey.

Northwest Power Planning Council. 1988. 1988 Update of the 1986 Northwest Power Plan, Volume 1. Portland, Oregon.

Peters, M. S., and K. D. Temmerhaus. 1980. Chemical Engineering Series. 3rd Edition. McGraw-Hill Book Company, New York.

Ross, B. A. June 1988. "Stirling Machines: From Potential to Practicality." Mechanical Engineering 11(6):34-42.

Strobridge, T. R. 1974. Cryogenic Refrigerations - An Update Survey. NBS TN-655, National Bureau of Standards, Washington, D.C.

Superconductor Industry. 1991. Fall 1991 Issue, p. 7. Rodman Publishing Corporation.

Weast, R. C. 1983. Handbook of Chemistry and Physics. 64th ed. CRC Press, Inc., Boca Raton, Florida.

Westinghouse Electric Corporation. 1982. Application of Low Temperature Technology to Power Transformers. DOE/ET/29324-1, U.S. Department of Energy, Washington, D.C.

Wharton Econometric Forecasting Associates (WEFA). 1987. Energy Analysis Quarterly. Bala Cynwyd, Pennsylvania.

Wilkinson, K.J.R. 1966. "Prospects for the Employment of Superconductors in Alternating Magnetic Fields." IEEE Transactions 2:369-374. 
Appendix A

Study Advisory Group 


\section{Appendix A}

\section{Study Advisory Group}

PNL formed a Study Advisory Group (SAG) to guide and support the HTS transformer study reported here. A listing of member organizations, including equipment manufacturers, utilities, and academia, is shown in Table A.1. The group's purpose was threefold: 1) to provide expert guidance to our researchers and analysts conducting the study, 2) to serve as reviewers of study products, and 3) to provide a forum for disseminating results of the study and identifying issues needing further research.

Table A.1. Systems Study Advisory Group

- Bonneville Power Administration (W. E. Myers) ${ }^{(a)}$

- Combustion Engineering Inc. (D. N. Palmer) ${ }^{(a)}$

- Electric Power Research Institute (F. S. Young) ${ }^{(a)}$

- General Electric Company (T. A. Keim)

- Massachusetts Institute of Technology (J. L. Kirtley)

- Ontario Hydro Research (F. Chu) ${ }^{(a)}$

- Pacific Gas and Electric Company (T. P. Mauldin) ${ }^{(a)}$

- Pacific Power and Light Company (J. H. Lambert) ${ }^{(a)}$

- Puget Sound Power and Light Company (B. C. Thomas)

- Southern California Edison (K. Holte) ${ }^{(a)}$

- Virginia Electric Power Company (J. Loncoski) ${ }^{(a)}$

- Washington State University (C. C. Mosher)

- Western Area Power Administration (T. L. Weaver) ${ }^{(a)}$

- Westinghouse Electric Corporation (C. J. Heyne) ${ }^{(a)}$

- W. J. Schafer Associates (S. M. Schoenung)

(a) Other individuals in these organizations were involved according to specialty. 


\section{Appendix B}

\section{Transformer Design Model Formulation}




\section{Appendix B}

\section{Transformer Design Model Formulation}

To adequately address all the issues related to transformer design and performance discussed in Chapter 3, it was necessary to develo and implement a computerized algorithm. The goal of the algorithm was to design viable transformers, from a set of materials-characteristic data using either conventional or superconductive technology. The code trades off all important design criteria and yields detailed information on material requirements and performance for various transformer designs. Finally, it provides, as output, a range of viable but not optimized transformer designs that meet a minimum performance screen. Output in this format allows detailed analysis of the issues involved in the transformer designs and further screening for optimization.

A code meeting these specifications was developed at PNL, and is called XFMR. A transformer design and performance analysis tool, XFMR is used to compare large conventional power transformers with corresponding superconductive designs. To ensure accurate technological assessment, similar design specifications are used when making comparisons.

This appendix contains a discussion of conventional transformer design methodology used in the model, followed by the superconductive design methodology. A detailed account of the equations used was provided in Chapter 3 of the text.

\section{B.1 Conventional Transformer Design Methodology}

The XFMR code is configured in such a way that the core flux density and the core dimensions are varied over a range of values to determine the design having the lowest life-cycle cost.

The first step involves calculation of the winding voltages and currents. This calculation is dependent on the power and voltage ratings of the transformer, the phase configuration (delta or wye) of the windings (if three-phase), and whether the transformer is an autotransformer.

Mean voltage insulation thicknesses for the various areas of the transformer are calculated based upon the estimated voltage stresses and the dielectric strength of the insulation. To determine the effective window area available for the conductors, the amount of insulation required is subtracted from the core window area available for each design. The core window dimensions are varied by the code based on input parameters that control the steps taken in the incremental design process.

The peak magnetic flux density level in the core, $B_{m}$, is incrementally varied. The allowable ranges for $B_{m}$ are 0.6 to $1.8 \mathrm{~T}$ for $12-\mathrm{mil} \mathrm{M}-5$ grain oriented silicon steel and 0.8 to $1.4 \mathrm{~T}$ for 2605S-2 METGLAS amorphous core alloy. These flux density ranges represent the intervals of validity for the core loss and permeability equations; these equations are derived from manufacturers' data. Core loss, core permeability, and leakage fields were calculated using equations that 
represented the best fit curves from manufacturing data. The data used, as well as the specific equations, are documented in Chapter 3.

For a given peak magnetic flux density level, the core iron loss is calculated in watts per kilogram of material. The peak magnetizing force, $\mathrm{H}_{\mathrm{o}}$, in amperes per meter; the leakage flux density, $\mathrm{B}_{\mathrm{L}}$; and the leakage loss, in watts per kilogram, are also calculated under the assumption that $\mathrm{H}_{\mathrm{o}}$ is approximately uniform throughout the transformer.

Core cross-sectional areas are next varied incrementally at each flux density level. The number of turns on the transformer primary and secondary windings are determined from Faraday's law, using the core area, flux density, phase voltage, frequency, and core stacking factor.

The width and height dimensions of the core window are varied over a specified range. The portion of the core window area occupied by insulation is determined from the required insulation thicknesses previously determined. The remaining window area is available for windings, cooling channels, etc. In our model, we did not perform a detailed thermodynamic analysis to determine the specific cooling requirements and channels necessary to cool the transformer. Rather, we allow sufficient space in which to locate the cooling passages. This space allowance is provided as an input variable called the "window utilization factor."

In the optimal design, the portion of the window area occupied by the primary and secondary windings may not be identical. A final code loop is included to vary the portion of the available window area occupied by each of the windings. This allows the analysis to determine the optimum thicknesses and amounts of materials allocated to each of the two windings for each phase.

The cross-sectional areas of the windings are next calculated from the effective window area available, the number of winding turns, and the window utilization factor input. This cross-sectional winding area is important for determining the resistance of the conductor, which will significantly drive the load losses of the transformer.

The mean-length-of-turns (MLT) for each transformer winding is then calculated, and with the number of turns per winding, the total length of each coil is determined. From the lengths and cross-sectional areas, along with the resistivity specified for conductor material, the electrical resistance of the windings is computed.

The leakage reactance of each transformer design is calculated by determining the effective leakage flux path length from the configuration of each transformer and from the leakage flux density.

The full-load $I^{2} R$ losses of the transformer are computed as the sum of the $I^{2} R$ losses on each of the three phases for both the primary and secondary windings.

No-load losses are then computed for each transformer design. These losses include the core iron loss, the stray losses due to leakage flux, the coil eddy-current losses, and the dielectric losses in the winding voltage insulation. The equations for these losses are given in Chapter 3.

The sum of the individual transformer losses is used to determine the full-load efficiency of the transformer. The efficiency is simply the output power of the transformer divided by the input 
power. Transformer designs with efficiencies greater than a minimum threshold (established in the input file) are saved in an output data file for further screening by the postprocessing program.

\section{B.2 Superconductive Transformer Design Methodology}

The superconductive transformer design methodology shares many algorithms with the conventional design process, with a few important differences. The first major difference is that the conductor current density is fixed in the superconductive design. Thus, for a given number of winding turns, the effective window area of the transformer is established. Therefore, the superconductive transformer design algorithm determines the area of the core window occupied by the conductor, insulation, and coolant space, then varies the width to height ratio of the core window, keeping the window area constant.

The second area of departure from the conventional transformer design is the requirement for stabilizer metal in cryostable transformer designs. The required amount of stabilizer metal is determined in a cryostability subroutine. The cryostability subroutine makes use of the "Stekly Stability Criterion." This relationship is essentially a heat balance equation and ensures that the amount of heat removed from the conductor during a quench is at least as much as the heat generated by resistive losses. As a result, the conductor temperature will remain well below the critical temperature, so a return to superconductivity is imminent upon removal of the quench-initiating overload. The cryostability subroutine calculates the amount of stability metal that must be included in the conductor for a cryostable transformer design according to the Stekly model.

Some of the loss mechanisms in the superconductive transformer also differ from the conventional. As mentioned previously, there are zero load losses in the superconductive transformer, but there are additional no-load losses.

In the superconductive transformer design methodology, the losses due to hysteresis in the superconductor and thermal influx into the cryostat must be included.

The impact of losses inside the cryostat is much greater than that of losses outside the cryostat. This is because all of the losses within the cryostat (hysteresis, eddy currents, dielectric, and heat leak) must be removed by the cryocooler and, thus, the effect of these losses is magnified by the low efficiency of the cryocooler.

\section{B.3 Input Data Requirements}

A large number of variables are involved in designing a transformer, either superconducting or conventional. Some of these variables can have dramatic effects on the overall design configuration, shape, size, efficiency, and so forth. Some variables are free to be changed and, within certain limitations, the variable can be randomly assigned in an effort to achieve the optimal design. This aspect of transformer design has been incorporated into this program; a number of loops are present that generate several designs when these parameters vary over a selected range of values. Other parameters are more constrained; their value depends on the available technology and engineering and 
manufacturing limitations. To ensure that they are reasonable and achievable, these values are selected based on sound engineering judgment, comparison with other transformer designs, and calculations.

In the following subsections, a description is given for each variable name that appears in the input file. The parameter and the basic relationships between variations in the parameter to changes in the overall design are briefly described.

\section{B.3.1 Design Specifications}

These variables define the design goals of the transformer. In addition to the power rating of the device and the voltage ratings of the windings, XFMR can generate designs for any of the following two winding transformers:

- superconductive (cryostable or ultrastable) or conventional windings

- single-phase or three-phase transformer design (three-phase winding configuration wye or delta for each winding)

- step-up/down or autotransformer

- standard silicon-steel or amorphous metal core.

A compete listing of the design specification variables in the input file is given here:

Phi Number of Transformer Phases

Three-phase or single-phase transformer.

kVl Primary Voltage (kV RMS)

kV2 Secondary Voltage (kV RMS)

Together these determine the turns ratio. Also, allowance for more insulation clearance is included for higher voltages.

MVA Transformer Power Rating (MVA)

Used in conjunction with the winding voltage ratings to determine the amount of current that flows in the windings, which affects the amount of conductor required.

$\mathrm{Hz} \quad$ System Frequency $(\mathrm{Hz})$

Assumed to be $60 \mathrm{~Hz}$, the system frequency affects the number of turns required by each winding. Several losses also depend on frequency. 
EFF

EffMin

BIL

FSafe

Mi

Wind $1 \quad$ Primary Configuration

Wind2 Secondary Configuration

Together these constitute the configuration of the windings and may be either WYE or DELTA. This will determine the voltage and current on the actual windings based on the external ratings of the transformer.

Status Technology

Can be either CONVENTIONAL or SUPERCEE. This will determine whether the conventional or the superconductor equations and design methodologies are used.

SCSafety Superconductor Protection

Can be ULTRASTABLE or CRYOSTABLE. With the cryostable design, a stabilizer metal matrix is assumed, and a routine is used to determine the amount of copper necessary to ensure a stable design. Ultrastable designs assume no stabilizer metal and will rely on an adequate margin given in FSafe to ensure stability. 
Can be LAMINATED or AMORPHOUS. There are separate values for core loss, maximum flux density, leakage reactance, and permeability for the two types of core materials.

Type Application

Can be STANDARD or AUTO-XFMR. This determines the current and voltage for each winding based on the external unit ratings and whether the transformer is isolated or connected as an autotransformer.

\section{B.3.2 Materials Specifications}

The superconductor specifications are included here that influence the winding losses. The ac losses in a multifilamentary superconductor depend on the manner in which the conductor is fabricated. The parameters that influence these losses are based on materials specifications given in this section.

The ac loss expressions used in this model are derived from low-temperature superconductor behavior. High-temperature superconductors may exhibit similar losses. However, the fabrication of ceramic superconductors may be considerably different than fabrication of the metals that formed the basis for the low-temperature superconductor configuration. Therefore, in an actual transformer using high-temperature ceramic superconductors, these parameters may not be valid, but configurations that produce similar losses can be used.

$\mathbf{J}_{\mathrm{c}}$

Critical Current Density (Base Case $=10^{5} \mathrm{~A} / \mathrm{m}^{2}$ )

Current the superconductor can carry (at $77 \mathrm{~K}$ and in the internal magnetic field of the transformer) before reverting to its normal state. This will determine the total conductor cross-sectional area required for superconductive transformer designs, with the direct effect of determining the size of the unit. Also, the hysteresis loss is directly proportional to $\mathrm{J}_{\mathbf{c}}$.

Lp Filament Twist Pitch (Base Case $=2 \mathrm{~cm}$ )

Eddy-current losses depend on the twist pitch length, which is the distance in the conductor before the filaments are completely transposed spirally.

D Superconductive Filament Diameter (Base Case $=50 \mu \mathrm{m}$ )

Generally constrained by manufacturing limitations; a smaller diameter results in lower hysteresis losses. 
Constrained by manufacturing limitations; a smaller radius results in reduced eddycurrent losses in the stabilizer metal in a cryostable superconductive transformer.

s

Conventional Conductor Strand Diameter (Base Case $=5 \mathrm{~cm}$ )

Used to calculate the eddy-current losses in the conductors of conventional transformers.

\section{B.3.3 Physical Constants}

These variables are determined by the operating environment of the transformer and depict the actual expected conditions. A complete listing of the input variables that are physical constants are given below:

QDOT Superconductor Cooling Rate/Unit Area $\left(20 \mathrm{~W} / \mathrm{cm}^{2}\right)$

This is the ability of the cooling medium to remove heat from the superconductor stabilizer metal. This value is used in determining the amount of copper needed for a cryostable design.

RhoCu77 Resistivity of Copper at $77 \mathrm{~K}\left(1.3 \times 10^{-8} \mathrm{ohm}-\mathrm{m}\right)$

Used in the calculation of the cryostability material requirements and eddy-current losses in the cryostable transformer. This value may be changed to reflect different materials used in the windings.

RhoCu320 Resistivity of Copper at $320 \mathrm{~K}\left(2.0 \times 10^{-8} \mathrm{ohm}-\mathrm{m}\right)$

Used to determine the load losses and eddy-current losses in the conventional transformer. Once again, it may be altered to reflect different conductor materials.

DensFe Density of Core Iron $\left(7.8 \times 10^{3} \mathrm{~kg} / \mathrm{m}^{3}\right)$

DensCu Density of Copper $\left(9.0 \times 10^{3} \mathrm{~kg} / \mathrm{m}^{3}\right)$

DensSC Density of Superconductor $\left(3.95 \times 10^{3} \mathrm{~kg} / \mathrm{m}^{3}\right)$

DensOil Density of Dielectric Fluid $\left(9.0 \times 10^{2} \mathrm{~kg} / \mathrm{m}^{3}\right)$

InsDens Mean Insulation Density $\left(1.7 \times 10^{3} \mathrm{~kg} / \mathrm{m}^{3}\right)$

These densities determine the weight of materials based on the calculated respective volumes. The weights are used to calculate the costs of the various materials. 
Er

\section{CUSC}

CUCONV

SFLam

SFAmorph

COP

Tamb

Tcryo
The voltage gradient designed into the transformer. A lower value will increase reliability but will also result in larger insulation clearances and, thus, higher material requirements and capital costs.

\section{Effective Relative Permitivity of Insulating Material (3.75)}

This determines the extent of dielectric material shielding against electric fields. Used in computing dielectric losses.

Window Utilization Factor - Superconductor (0.75)

Window Utilization Factor - Conventional (0.95)

Fraction of the effective core window that is active conductor. Consideration must include spacing between turns, structural supports for the windings, and any cooling passages that are necessary to remove heat from the windings. The conventional utilization factor should be higher than the superconductor utilization factor resulting from the increased cooling requirements for a cryogenic operating system.

\section{Laminated Core Stacking Factor (0.96)}

\section{Amorphous Core Stacking Factor (0.82)}

The core stacking factor determines the difference in real volume (necessary for window dimensions and weight considerations), and effective magnetic volume (used when calculating core radius and flux density). The core is constructed of thin sheets of magnetic material with very thin insulation layers. Over the aggregate volume of the core volume, these small insulated spaces can account for as much as $10 \%$ to $20 \%$ of the total core volume.

\section{Refrigeration Coefficient of Performance}

For every thermal watt produced in the cryostat, a refrigeration system must remove it, with a penalty associated with the energy requirements to run the refrigeration system. This becomes important if there are high losses that must be removed by the refrigeration system. The COP is a function of the operating and ambient temperatures, as well as the quantity of heat removed. If a value of 0 is entered, the COP is calculated based on the equation in Chapter 3, which is based on operating the cryostat at $77 \mathrm{~K}$.

Maximum Temperature External to Cryostat (300 K)

Cryostat Internal Temperature (77 K) 
These parameters are used to calculate the heat leak into the cryostat. Other, separate factors, such as resistivity and COP, depend on temperature.

Tc

Superconductor Critical Temperature (K); no specific value, but greater than Tcryo.

This is the temperature at which the superconductor reverts to its normal state. A check is made to ensure that the specified Tc is greater than Tcryo.

Stress

Dielectric Stress $\left(1.65 \times 10^{6} \mathrm{~V} / \mathrm{m}\right)$

This is the allowable voltage gradient for the insulating material; it is used to calculate the dielectric losses.

LossTang Mean Loss Tangent of Insulation Material $\left(2 \times 10^{-4}\right)$

Used for calculating dielectric losses in the insulating medium.

\section{B.3.4 Control Variables}

The final set of variables contained in the input file is control variables that control the operation of the program. Because the program steps through a range of designs, the initial values, final values, and resolution of the steps are specified. These variables do not directly influence the design except in determining the fundamental range of designs investigated. By retaining a high degree of freedom in the control variables, the program can readily converge on optimum designs quickly and accurately.

BmMin $\quad$ Minimum Core Peak Magnetic Flux Density (T)

BmMax Maximum Core Peak Magnetic Flux Density (T)

BmStep Core Peak Magnetic Flux Density Increment (T)

The magnetic flux density is one of the parameters for which variation is necessary to achieve the optimal design. The user defines the range and sensitivity of the variation, and the program will give a design for each of these values.

rCoreMin Minimum Core Radius (m)

rCoreMax Maximum Core Radius (m)

CoreStep Core Radius Increment (m)

The core radius is a variable that also must be analyzed over a range of possible values to determine the optimum design. Once again, the range and refinement desired are given with designs computed for each value. 


\section{HWindMin Minimum Core Window Height (m) \\ HWindMax Maximum Core Window Height (m) \\ HghtStep Core Window Height Increment (m)}

These variables are for variation of the core window height, which is also an independent variable that must be fully analyzed over a range to determine the optimum.

BWindMin Minimum Core Window Base Width (m)

BWindMax Maximum Core Window Base Width (m)

BaseStep Core Window Base Width Increment (m)

These variables are similar to the core window height. In a conventional design, they are used to determine the window area. After insulation and utilization factors are allowed for, the window area is used to determine the conductor area, which, in turn, determines the current density and the losses. In a superconductor design, the operating current density is fixed by the material, so the conductor area and the total window area are fixed. Therefore, the window base is dependent on the height, so a window width-to-height ratio is used, which is varied over a range of selected values based on information given in the above six variables.

PDivMin Minimum Primary Window Area Fraction

PDivMax Maximum Primary Window Area Fraction

PStep Window Area Fraction Increment

Applicable only to conventional transformers, the optimal use of the window space depends on what fraction of the window area is occupied by the primary conductor.

LLIMIT Maximum Transformer Length (m)

WLIMIT Maximum Transformer Width (m)

HLIMIT Maximum Transformer Height (m)

These variables are the maximum dimensions of the transformer. The program runs by stepping through many parameters; thus, unreasonable designs are possible. These constraints are used to flag designs that exceed the maximum allowable dimensions and are set to reflect transportation limitations. 


\section{B.4 Output Parameters and Data Format}

The output of the code includes all designs that are specified by the input design parameters and that meet the minimum efficiency requirement. The information in the output includes the transformer full-load efficiency, the weights of the various components, dimensional information relating to the size of the unit, and a complete breakdown of all power losses. This information is then used to analyze the life-cycle costs of the various transformer designs.

The basic output file can be divided into two distinct groups: the header and the performance information. The header contains all of the important identifying information regarding the run. Most of the key design specification variables are reflected in this information. The second portion of the output file is the performance information for each specific design that meets the minimum efficiency threshold.

The header occupies three lines of code containing the following information:

- power rating of the transformer in MVA

- terminal voltage ratings of the transformer in $\mathrm{kV}$

- number of phases

- winding configuration (valid for three-phase units)

- core type (amorphous or laminated)

- transformer type (conventional, cryostable, or ultrastable)

- autotransformer or step-up/down transformer.

The performance information contains the following information for each potentially viable transformer design:

- full-load efficiency

- core peak magnetic flux density

- core leg radius

- core window height

- core window base length

- portion of core window for primary windings

- total core weight 
- total copper weight

- core loss

- full-load conductor loss

- cryostat heat leak

- hysteresis loss in the conductor

- eddy-current losses in the conductor

- leakage loss

- dielectric loss

- total thermal losses

- total electrical losses

- refrigeration COP

- transformer insulating oil weight

- transformer tank weight

- transformer total length

- transformer total width

- transformer total height

- oversize flag

- number of primary turns

- number of secondary turns

- length of the primary conductor

- length of the secondary conductor

- volume of the primary conductor

- volume of the secondary conductor

- cross-sectional area of the primary conductor 
- cross-sectional area of the secondary conductor

- insulation clearance for ground to primary winding

- insulation clearance for ground to secondary winding

- insulation clearance between primary and secondary winding.

Some of these variables are redundant, and some are not necessary to determine the life-cycle cost of the various designs. However, they are included to allow "reasonability checks" to ensure that the code is operating properly. These output parameters are stored in an output file, which a post-processing program uses.

\section{B.5 Post-Processor Program Description: Purpose, Approach, Operation}

A post-processor utility program was written to allow intelligent screening of transformer designs output by XFMR, to remove designs that are redundant or obviously less than economically efficient. The post-processor is designed to sort the output of the XFMR code by efficiency and material quantities. Of two transformer designs having the same efficiency, the one with more conductor and core material is dropped while the other design is retained for further consideration. If two transformer designs have the same efficiency and one has more conductor material but less core material, both designs are saved, because no prior decision can be made as to which one has the lower life-cycle cost. This process is carried out for all initial output designs to minimize the amount of data used in the final stages of optimization.

The screened data file is organized with a format that is compatible with the transformer design economic optimization code (discussed in Chapter 5 of this report). 
Appendix C

EAST Code Description 


\section{Appendix C}

\section{EAST Code Description}

The EAST model (Economic Analysis of Superconducting Technologies) was developed to provide the mechanism for integrating the information from the design and cost models. For a given transformer type, EAST reads in a number (typically on the order of a thousand) of alternative transformer designs from the output files of the design code. The model calculates the life-cycle cost of each design. The designs are then sorted in order of increasing life-cycle cost. A functional description of the model is provided below.

\section{C.1 Implementation}

EAST runs on an IBM personal computer (PC) and was developed using Lotus 1-2-3. Data input/output and calculations are controlled by a Macro file (developed using Superkey software) that contains the several hundred Lotus commands required to process the data.

While EAST could conceivably run on any PC that could operate Lotus, our experience with the code indicates that optimization runs for transformers are most efficient if the PC has a minimum of 2 megabytes of random access memory (RAM).

\section{C.2 Code Logic}

The logic in EAST is simple and straightforward. The code operates as follows:

- Data Input - Data are read from an ASCII file produced by the transformer design code. These data correspond to a number of different transformer designs for a given specific application. Data used by the code are described in the next section.

- Cost Estimation - For each of the transformer designs, EAST estimates the capital cost, O\&M cost, and costs of annual energy losses. The life-cycle cost impacts from each of these categories are then estimated and combined into a total transformer life-cycle cost for each design.

- Design Sorting - After the life-cycle costs have been estimated, the transformer designs are sorted in order of ascending life-cycle cost to identify the most cost-effective designs evaluated.

- Data Output - Code results are written to an ASCII and Lotus file for later evaluation. 


\section{C.3 Transformer Design Data}

The EAST code uses the following information provided by the transformer design code:

- Design Type - a single variable name that identifies the following:

- transformer type (HTS-cryostable, HTS-ultrastable, or conventional)

- the type of core material (amorphous or Si-steel)

- the MVA rating of the transformer

- ID Number - This provides a unique design identifier that matches output from the design code to output from the EAST code.

- Shell Weight - The shell weight in $\mathrm{kg}$ is used to estimate the capital cost of the transformer shell.

- Core Weight - The core weight in $\mathrm{kg}$ is used to estimate the capital cost of the transformer core.

- Copper Weight - The copper winding weight in $\mathrm{kg}$ is used to estimate the capital cost of copper transformer windings.

- HTS Weight - The weight in kg of HTS windings is used to estimate the capital cost of HTS transformer windings. For the composite windings used in cryostable designs, EAST uses both copper weight and HTS weight in developing a cost estimate for the composite winding cost.

- Cryostat Input Power - The input power in $\mathrm{kW}$ required for the cryogenic cooler is used by EAST to estimate the capital cost of the cryocooler.

- Oversize Shipping - This flag identifies any transformers that would require special shipping considerations because of mass or dimensions.

- Load Losses - Transformer load losses in kW for operating at rated capacity are used in estimating the annual cost of energy losses due to load losses.

- No-Load Losses - Transformer no-load losses in $\mathrm{kW}$ are used in estimating the annual cost of energy losses due to no-load losses.

- Downtime Losses - This variable gives average losses in $\mathrm{kW}$ for operating the cryogenic system of HTS step-up transformers when the power plant is not operating. This variable is used to estimate the annual cost of downtime energy losses. 


\section{C.4 Transformer Capital Cost Estimation}

EAST estimates the transformer capital cost by aggregating cost estimates for a number of individual cost elements. Each of the capital cost elements is discussed separately below. Data for the equations are shown in the next section.

- Shell Costs - As used in this analysis, the transformer shell represents not only the structural container for the core and windings, but also a general "miscellaneous" category to capture aspects of the transformer sales price not included in the windings and core components. Shell costs are estimated by

$$
\text { Shell Cost }=S C 1 *(\text { shell weight, } \mathrm{kg})^{\wedge} \mathrm{SC2}
$$

- Core Costs - Transformer core costs are estimated by

$$
\text { Core Cost }=C C 1 *(\text { core weight, } k g)^{\wedge} \mathrm{CC} 2
$$

- Windings Costs - Transformer windings may be all copper (conventional transformers), a mixture of copper and HTS material (HTS-cryostable), or pure HTS material (H'TS-ultrastable). The transformer windings cost is estimated by

$$
\begin{array}{rl}
\text { Windings Cost }= & W C 1 *(\text { copper weight, } k g)^{\wedge} W C 2+ \\
W C 3 & W(\text { HTS weight, } k g)^{\wedge} W C 4
\end{array}
$$

- Cryocooler Costs - Cost for the cryogenic refrigeration system are estimated by

$$
\text { Cryocooler Cost }=C R I *(\text { cryocooler input power, } k W)^{\wedge} C R 2
$$

- Shipping Costs - Shipping costs are based on the total transformer weight in kg. Shipping costs are summarized below.

$$
\begin{gathered}
\text { Shipping Weight, kg } \\
0-26,999 \\
26,999-67,999 \\
>67,999
\end{gathered}
$$

Shipping Cost, $\$ K$
2
100
200

- Installation - Costs for transformer installation are based upon the total transformer weight (combined weight of shell, core, and windings) according to

$$
\text { Installation Cost }=I C 1 *(\text { total transformer weight, } \mathrm{kg})^{\wedge} I C 2
$$




\section{C.5 Transformer O\&M Costs}

Transformer O\&M costs are separated into O\&M for the cryocooler and O\&M for all other parts of the transformer. Annual O\&M for the cryocooler is estimated at 5\% of the initial cryocooler capital costs. O\&M for all other parts of the transformer is estimated at $2 \%$ of the capital cost (including installation) for those components.

\section{C.6 Transformer Annual Energy Costs}

Costs for annual energy losses are handled differently for grid and step-up transformers. For grid transformers, energy losses are broken into load losses and no-load losses. No-load losses continue throughout the year and are estimated as

$$
\text { Annual No-Load Losses }=8760 *(\text { no-load loss, } k W)
$$

Annual energy costs for the no-load losses are estimated by multiplying the annual losses by the value of energy for no-load losses.

Load losses for transformers are estimated by an empirical relationship that accounts for the variability of transformer load throughout the year and the fact that load losses vary with the square of the transformer current. Annual load losses are estimated as

$$
\text { Annual Load Losses }=8760 * \text { loss factor * (rated load losses, } k W)
$$

The Loss Factor is calculated as

$$
\text { Loss Factor }=A *(\text { load factor })+B(\text { load factor })^{\wedge} 2
$$

The annual energy costs for the load losses are estimated by multiplying the annual losses by the value of energy for load losses.

For step-up transformers, energy losses are broken into operating losses and (for HTS transformers) into nonoperating losses. Annual operating losses are estimated as

$$
\text { Annual Operating Losses }=8760 * \text { (plant capacity factor }) *(\text { loss, } \mathrm{kW})
$$

The annual energy costs for the operating losses are estimated by multiplying the annual losses by the value of energy for operating losses. 
HTS transformers require energy during nonoperational times to maintain the temperature in the cryostat. Annual energy losses for downtime periods are estimated as

$$
\begin{aligned}
\text { Annual Downtime Losses }=8760 *(\text { avg. cryocooler power, } k W) * \\
(1-\text { plant capacity factor })
\end{aligned}
$$

The annual energy costs for the downtime losses are estimated by multiplying the annual losses by the value of energy for downtime losses.

\section{C.7 Life-Cycle Costs}

The transformer life-cycle cost is calculated as the net present value of owning and operating the transformer over its life. Present value factors were calculated using the PNL model TEAM (Brown et al. 1987) to convert the initial and annual costs into present value terms. The equations used are

$$
\begin{gathered}
\text { PV Capital Costs }=(\text { initial capital cost }) * P V C \\
P V \text { Annual O\&M Cost }=(\text { first year O\&M cost }) * P V O \\
\text { PV Annual Losses }=(\text { annual loss, } k W h) * P V L
\end{gathered}
$$

Different values are required for PVL depending upon the type of energy loss involved.

\section{C.8 Model Input Data}

The base-case cost data used for the HTS and conventional transformers are shown in Tables C.1 and C.2. Data for energy values and present value calculations in the base-case analyses are shown in Table C.3. 
Table C.1. Cost Model Input Data for HTS Transformers (all costs in 1989 \$, thousands)

\begin{tabular}{|c|c|c|c|c|}
\hline Shell Cost & Core Cost & Windings Cost & Cryocooler Cost & $\begin{array}{c}\text { Installation } \\
\text { Cost }\end{array}$ \\
\hline$S C I=0.0527$ & Amorphous Cores & $\mathrm{WC1}=0.1159$ & $\mathrm{CR} 1=12.8645$ & $\mathrm{ICl}=0.9165$ \\
\hline \multirow[t]{4}{*}{$S C 1=0.7766$} & $\mathrm{CCI}=0.1288$ & $W C 2=0.8482$ & $\mathrm{CR} 2=0.7351$ & $\mathrm{IC} 2=0.2977$ \\
\hline & Si-Steel Cores & WC3 $=1.628$ & & \\
\hline & $\mathrm{CCI}=0.0917$ & $\mathrm{WC4}=0.8525$ & & \\
\hline & $\mathrm{CC} 2=0.7739$ & & & \\
\hline
\end{tabular}

Table C.2. Cost Model Input Data for Conventional Transformers (all costs in 1989 \$, thousands)

\begin{tabular}{|c|c|c|c|c|}
\hline Shell Cost & Core Cost & Windings Cost & Crycooler Cost & $\begin{array}{c}\text { Installation } \\
\text { Cost }\end{array}$ \\
\hline$S C 1=0.0486$ & Amorphous Cores & $\mathrm{WC1}=0.1159$ & $\mathrm{CRI}=0.1$ & $\mathrm{IC} 1=0.9165$ \\
\hline \multirow[t]{5}{*}{$S C 2=0.7739$} & $\mathrm{CCl}=0.1288$ & $W C 2=0.8482$ & $\mathrm{CR} 2=1.0$ & $\mathrm{IC} 2=0.2977$ \\
\hline & $\mathrm{CC} 2=0.7730$ & $\mathrm{WC3}=0.0$ & & \\
\hline & Si-Steel Cores & $\mathrm{WC4}=1.0$ & & \\
\hline & $\mathrm{CCl}=0.0917$ & & & \\
\hline & $\mathrm{CC} 2=0.7739$ & & & \\
\hline
\end{tabular}


Table C.3. Energy and Present Value Factors

\section{Annual Energy Costs}

All values are levelized energy costs in constant 1989 dollars.

Grid Transformer Load Losses $=\$ 0.112 / \mathbf{k W h}$

Grid Transformer No-Load Losses $=\$ 0.066 / \mathrm{kWh}$

Peaking Step-Up Operating Losses $=\$ 0.094 / \mathrm{kWh}$

Intermediate Step-Up Operating Losses $=\$ 0.059 / \mathrm{kWh}$

Baseload Step-Up Operating Losses $=\$ 0.055 / \mathrm{kWh}$

Present Value Factors

$\mathrm{PVC}=0.92$

PVO $=10.22$

Present value factors for energy costs convert annual losses in $\mathrm{kWh}$ to present value of losses over the lifetime of the transformer. Values of PVL for various types of energy losses are shown below.

Grid Transformer Load Losses $=\$ 1.142 / \mathrm{kWh}$

Grid Transformer No-Load Losses $=\$ 0.676 / \mathrm{kWh}$

Peaking Step-Up Operating Losses $=\$ 0.962 / \mathrm{kWh}$

Intermediate Step-Up Operating Losses $=\$ 0.604 / \mathrm{kWh}$

Baseload Step-Up Operating Losses $=\$ 0.559 / \mathrm{kWh}$ 
Appendix D

HTS Market Assessment Program 


\section{Appendix D}

\section{HTS Market Assessment Program}

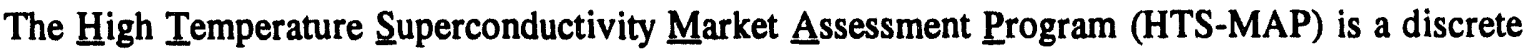
expected-value simulation code developed to estimate both the potential market penetration of superconducting transformers in the electric utility industry and the societal benefits of adopting superconducting transformers. Expected values and standard deviations are used from probability distributions rather than Monte Carlo sampling to determine the activities and states of the simulation as time is advanced in fixed-time increments.

The HTS-MAP structure is designed using modular architecture. It consists of a main program and 18 well-structured, modular subroutines. The subroutines are functionally specific to facilitate program modification and maintenance. All subroutines include indentation of code, liberal use of comments, judicious use of blank lines, and uppercase letters for variable names and lowercase letters for subscripts to improve readability. Each subroutine has a commentary prologue that describes the subroutine, its limitations, and other subroutines called. In addition, the prologue defines the author, coding date, compiler, compiler options, and modification history. Between subroutines data are transferred generally through 12 labeled storage blocks known as common blocks, which contain related variables and constants.

HTS-MAP was developed using Microsoft FORTRAN 77 version 4.0 on a Compaq 386 microcomputer with an 80387 coprocessor running under DOS version 3.1. The Microsoft compiler extension permits the use of variable names longer than six characters and was used throughout the code to give them mnemonic value and to facilitate understanding the meaning of the variable names. The program will run on any IBM-AT or compatible microcomputer having a coprocessor and at least 640 kilobytes of computer memory. The memory requirements are based on the current program dimensions, which allow a maximum lifetime of 40 years for transformers and up to 75 years for the length of the simulation.

\section{D.1 Running the HTS-MAP Program}

To execute HTS-MAP, the high-density diskette containing the program files and data files for HTS-MAP should be inserted in drive A of the computer and copied to a new directory of the hard disk (such as HTS). The command DEVICE=ANSI.SYS must be in the CONFIG.SYS file. Then from the appropriate drive/directory prompt, typing "HTS" < RETURN > will execute the DOS batch file HTSC.BAT. This file executes the FORTRAN executable program HTS and copies the input data files and simulation output to the DOS output file HTSC.PRT for later disposition to the printer.

The program uses throe iSCII input data files, labeled HTSC.DAT, TRANSPOP.DAT, and HCAPLERN.DAT. The file HTSC.DAT is the main input data file. It is organized into blocks of 
data that are specific to each module. The file TRANSPOP.DAT populates the initial inventory of conventional transformers at the start of the simulation by age and size. The file HCAPLERN.DAT defines the learning multipliers for multiplying the present value of long-term capital costs of superconducting transformers by size and years elapsed from initial market penetration.

The default values in any of the three files can be changed by consulting the Variable Dictionary and using a xt editor such as WordStar or WordPerfect to edit the files as ASCII DOS text files.

The output from the program is sent to a DOS file labeled HTSC.PRT. The three input data files are copied to the front of the output file to provide a record oi the input data used in the simulation. The output file is printed using a word processor such as WordPerfect, with left margin set to 12 and right margin set to 100 .

\section{D.2 Synopsis of HTS-MAP Logic}

Figure D.1 shows an overview of the principal activities performed by HTS-MAP in estimating the potential market penetration and societal benefits of HTS transformers in the electrical utility industry. The numbering scheme used for the activities in Figure D.1 was adopted to be consistent with the numbering scheme used in Chapter 7 to describe the methodology of the activities.

The tree diagram in Figure D.2 shows the modular architecture of HTSMAP. Each block in Figure D. 2 represents a specific subroutine. The number in each modular block identifies the principal activity performed by the subroutine from the list of activities shown in Figure D.1. These numbers map the subroutines to the principal activities performed by HTS-MAP.

The connecting lines represent calls to the subroutines. A subroutine calls another subroutine only if the latter is positioned lower in the block diagram and if there is a direct connection between the two subroutines. For example, the MAIN program calls subroutine SIGNON, which in turn calls subroutine CLRSCN. The MAIN program calls all of the subroutines shown in Figure D.2 in the order of the block numbers, with the exception of subroutines CLRSCN, CUM_NORMAL, SURVIVING_INVENT, PRT_MATRIX2D, and PRT_MATRIX3D. These subroutines are called by subroutines SIGNON, MKT_PENETR, BENEFITS, and OUTPUT, respectively.

The MAIN program calls the first row of subroutines once, starting with CLEAR and ending with HTSC_FAIL, to set up the starting conditions for the simulation. Next, the MAIN program calls the second row of subroutines starting with REPLACEMENTS and ending with UPDATE_INVENT sequentially in an iterative loop, beginning with the starting year of the simulation and proceeding each year thereafter to the final year of market penetration. These four subroutines are enclosed within a box to indicate that the MAIN program calls the same sequence of subroutines, REPLACEiviENTS, TOTAL_NEW_UNITS, MKT_PENETR, and UPDATE_INVENT, repeatedly during the simulation. This iterative loop forms the main body of the market penetration simulation. Finally, the MAIN program calls subroutines BENEFITS and OUTPUT each one time to complete the simulation. 


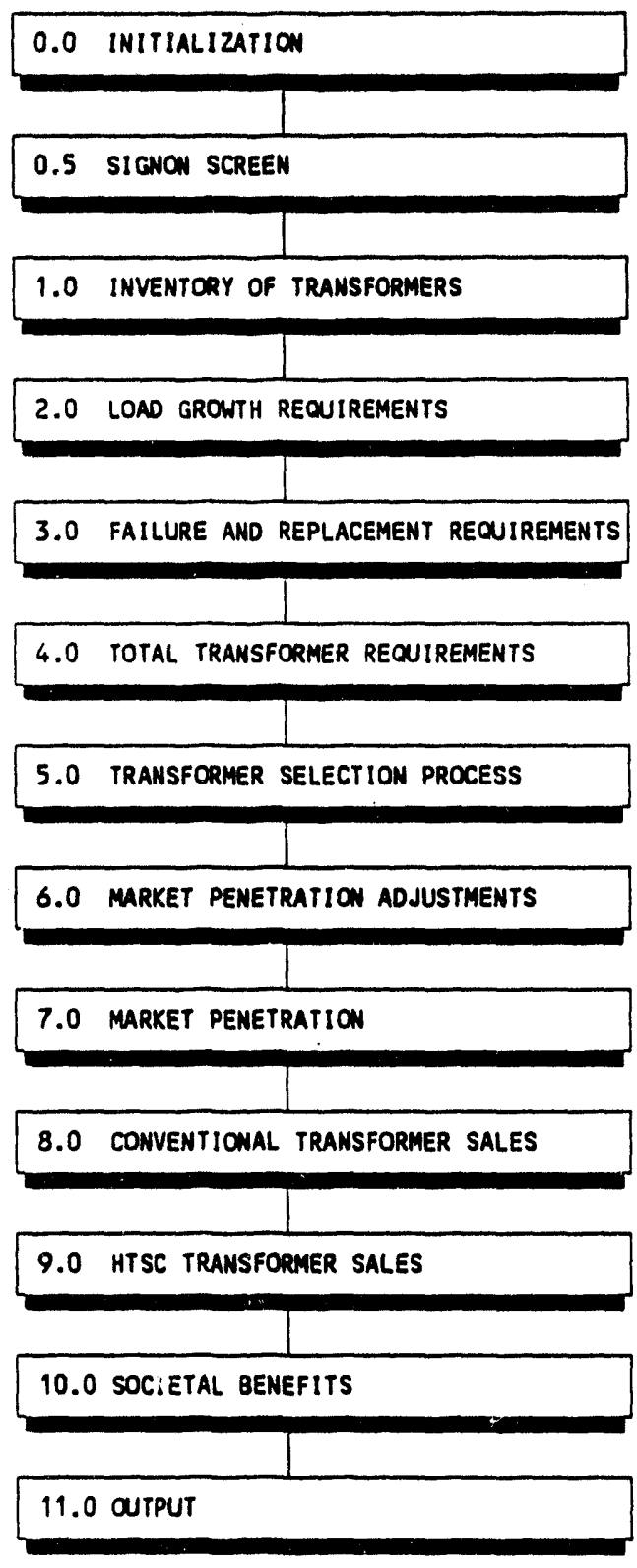

Figure D.1. Overview of Principal Activities in High-Temperature Superconductivity Market Assessment Program

\section{D.3}




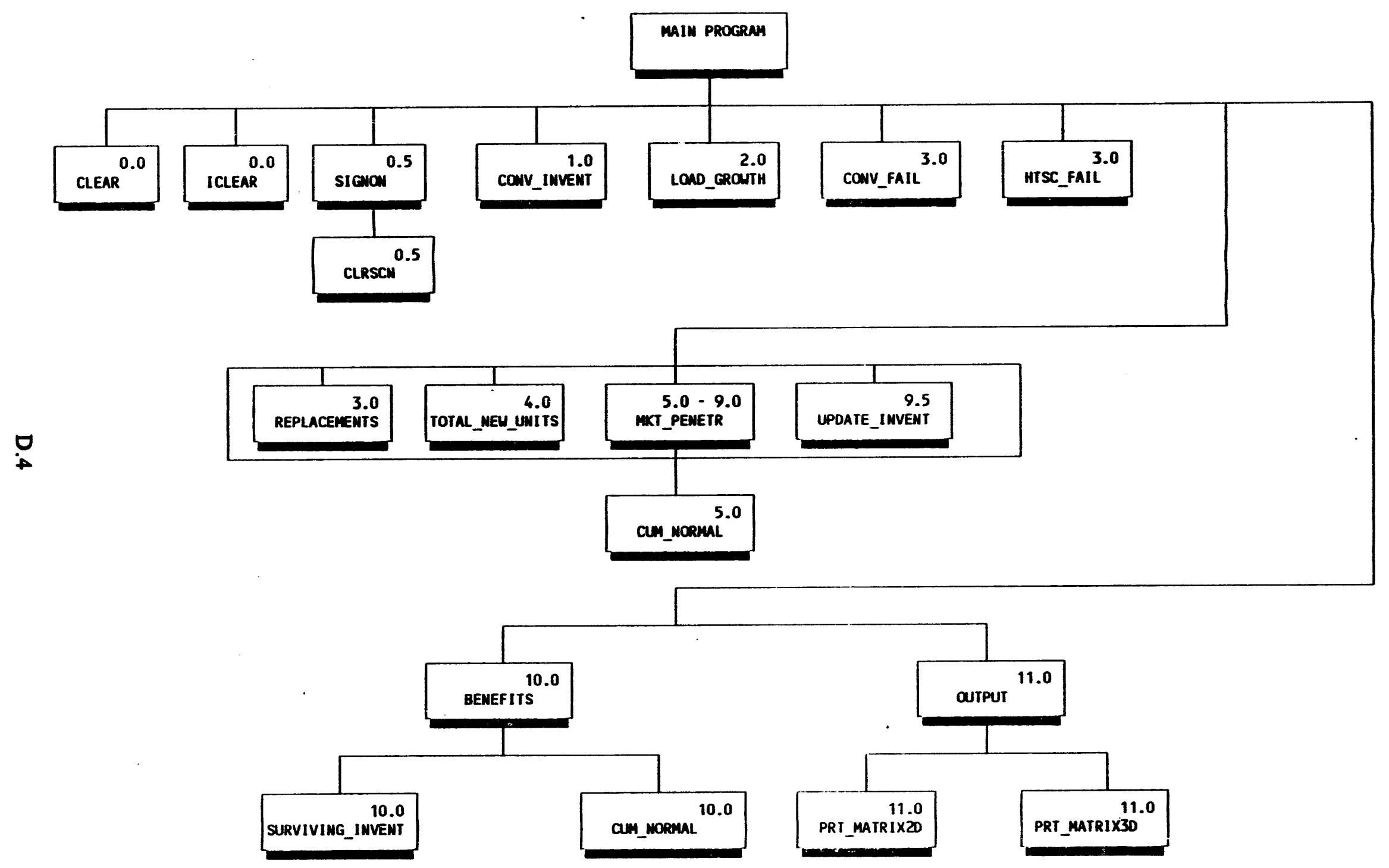

Figure D.2. Modular Architecture of High Temperature Superconductivity Market Assessment Program 
Each of the subroutines within the simulation code is briefly described below. The principal computational steps are summarized for each subroutine that implements the detailed methodology previously described in Chapter 3.

\section{D.2.1 Subroutine Descriptions}

The MAIN PROGRAM does the preliminary initialization, reads the three input data files, calls the subroutines shown in Figure D. 2 to process and control the simulation, and advances the simulation time. The Main Program simulates by advancing time in fixed-time increments of one year, beginning with the input starting time and by letting the events occur as dictated by the submodels (subroutines) in the simulation. The status variables in the simulation (e.g., the number of failures and retirements, the number of new transformers instailed, existing inventories) are updated at the end of every year.

- (0.0) CLEAR sets floating-point variables and arrays to zero in the 12 labeled common Llocks.

- (0.0) ICLEAR sets integer variables and arrays to zero in the labeled common blocks.

- (0.5) SIGNON displays the sign-on screen for the program by utilizing the ANSI.SYS device driver. This driver is an extended screen and keyboard driver that appears as a separate file on the DOS system disk. It allows the code to position the cursor on the screen while working in DOS, to set colors for various areas, and to display messages at specific points on the screen.

- (0.5) CLRSCN is called by SIGNON to clear the screen before displaying the sign-on screen. It also requires the ANSI.SYS device driver.

- (1.0) CONV_INVENT reads the TRANSPOP.DAT file to initialize the inventory of conventional auto and step-up transformers by size and age at the beginning of the simulation. These inventories are stored in the arrays CONV_AUTO_HIST_INVENT(isize, iage_cell) and CONV_STEPUP_HIST_INVENT(isize, iage-cell), respectively.

- (2.0) LOAD_GROWTH calculates the number of new auto and step-up transformers of each size required annually to meet load growth from the first year to the final year of the simulation. LOAD_GROWTH performs the following principal computational steps:

1. Computes the system peak load (MWe) at the beginning of the first year as a function of the summer peaking load (MWe) input for the first year and the input current reserve margin (fraction).

2. Computes the system peak load (MWe) at the end of the year input to reach the desired reserve margin as a function of the system peak load computed in Step 1 and the input projected load growth (fraction).

3. Calculates the desired system capacity (MWe) at the end of the year input to reach the desired reserve margin as a function of the system peak load calculated in Step 2 and the input desired reserve margin (fraction). 


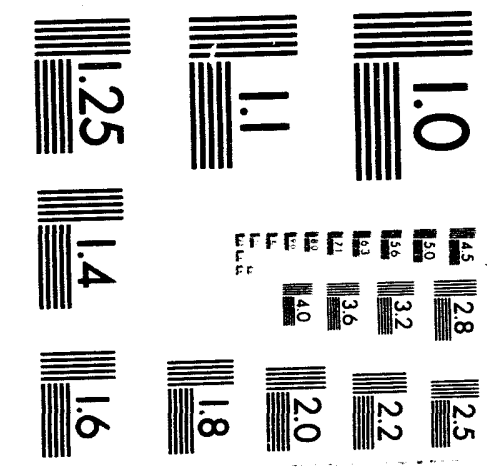




$$
\begin{aligned}
& \boldsymbol{\omega} \\
& \stackrel{\mathrm{o}}{\boldsymbol{\omega}}
\end{aligned}
$$


4. Calculates the projected capacity additions (MWe) required annually during the number of years input to reach the desired reserve margin by distributing the total additional system capacity calculated in Step 3 uniformly over the number of years to reach the desired reserve margin.

5. Lalculates the projected capacity additions (MWe) required annually from the end of the year following the year in which the desired reserve margin is reached to the final year of the simulation as a function of the projected load growth.

6. Determines the mix of sizes of auto and step-up transformers required annually to meet the load growth from the first year to the final year as a function of the annual projected capacity additions computed in Steps 4 and 5 and the input auto and step-up size distributions: AUTO_SIZE_DISTR(isize) and STEPUP_SIZE_DISTR(isize). These input distributions specify the number of auto and step-up transformers, respectively, required of each size to provide every $1000 \mathrm{MWe}$ of new capacity.

- (3.0) CONV_FAIL computes the fraction of conventional auto and step-up transformers that will be replaced in each age and size group over the course of a year. The replacements cccur as the result of both failures and retirements when the transformer reaches the maximur.t age input for the transformer type and size group. Separate Weibull distributions for each type of transformer (auto and step-up) and each size of transformer, defined by the input parameters for the location, scale, and shape of the Weibull distribution, are used to model the time to failure for conventional auto and step-up transformers in each size group. The basic equation used to compute the fraction of transformers that will need to be replaced over the course of a year is as follows:

$$
R E P F R=\begin{aligned}
& F(1.0), \text { for new transformers }(X=0) \\
& {[F(X+1)-F(X)] /[1 .-F(X)], \text { for } 1<X<M A X A G E-1} \\
& 1.0, \text { for transformers of maximum age }(X=M A X A G E)
\end{aligned}
$$

where $\quad$ REPFR $=$ the fraction of transformers of given type and size replaced

$F(X)=$ the cumulative distribution function for the Weibull distribution

$\mathrm{X}=$ the age (years) of the transformer

MAXAGE = the maximum age input for the transformer type and size group, after which it is retired.

The replacement probabilities are calculated at the beginning of the simulation and stored in the arrays CONV_AUTO_PFAIL(isize, iage_cell) and CONV_STEPUP_PFAIL(isize,iage_cell) for conventional auto and step-up transfọrmers, respectively.

- (3.0) HTSC_FAIL computes the fraction of superconducting auto and step-up transformers that will be replaced in each age and size group over the course of a year. The computational logic is analogous to that of subroutine CONV_FAIL but different Weibull distributions are used to model the time to failure for superconducting auto and step-up transformers. The replacement probavilities are calculated at the beginning of the simulation and stored in the arrays HTSC_AUTO_PFAIL(isize,iage_cell) and HTSC_STEPUP_PFAIL(isize.iage_cell) for superconducting auto and step-up transformers, respectively. 
- (3.0) REPLACEMENTS calculates the number of conventional and superconducting auto and step-up transformers in each size and age group to be replaced from the end of the previous year to the end of the current year. The replacements occur as the result of nth failures and retirements. REPLACEMENTS is called each time step by the MAIN p.ugram from the first year of the simulation to the final year of market penetration. The replacements are calculated by multiplying the inventory of transformers in each type, size, and age group existing at the end of the previous year by the fraction of replacements occurring annually in each type, size, and age group. The latter was computed by subroutines CONV_PFAIL and HTSC_PFAIL. Superconducting transformers are svailable for replacement only after the first year of market penetration. The number of conventional and superconducting auto and step-up transformers replaced is stored in the arrays: CONV_AUTO_REP (isize, iage_cell), CONV_STEPUP_REP (isize, iage_cell), HTST_AUTO_REP(isize, iage_cell), and HTSC_STEPUP_REP(isize, iage_cell).

- (4.0) TOTAL_NEW_UNITS computes the number of new auto and step-up transformers needed in each size group to meet load growth and to replace failures and retirements in each type, size, and age group. TOTAL_NEW_UNITS is called each time step by the MAIN program from the first year of the simulation to the final year of market penetration. The number of new auto and step-up transformers needed annually in eaci: size group are stored in the arrays AUTO_NEW(isize,iyr) and STEPUP_NEW(isize,iyr), respectively.

- (5.0-9.0) MKT_PENETR calculates the market penetration of superconducting auto and step-up transformers from the end of the previous year to the end of the current year. MKT_PENETR is called each time step by the MAIN program from the first year of the simulation to the final year of market penetration. Before the first year of market penetration, the total new transformers needed annually are selected from conventional transformers. In the first year of market penetration and subsequent years, the total new transformers needed annually are selected from a mix of conventional and superconducting transformers. MKT_PENETR performs the following principal computational steps in the market penetration algorithm for autotransformers:

1. Constructs a normal distribution for load energy costs by assuming that the ratio of standard deviation to mean is the same as that of the normal distribution for industrial energy prices from U.S. utilities taken from Energy User News.

2. Constructs a normal distribution for no-load energy costs using the same method as in Step 1.

3. Constructs a normal distribution for the combination of load and no-load energy costs for conventional transformers by calculating a weighted average of the means of the two normal distributions constructed in Steps 1 and 2 using the fraction of total losses that are input as load or no-load for conventional transformers. The standard deviation for the combined normal distribution is calculated by assuming that the ratio of standard deviation to mean is the same as that for industrial energy prices from utilities.

4. Constructs a normal distribution for the combination of load and no-load energy costs for superconducting transformers by combining the two normal distributions calculated in Steps 1 and 2 weighted by the fraction of total losses that are input as load or no-load for 
superconducting transformers. The standard deviation is calculated in an manner analogous to that in Step 3.

5. Solves for the "threshold" energy price, based on conventional transformers such that any utility with combined load and no-load energy costs greater than this value will select superconducting autotransformers when installing new autotransformers. This equation is described in Chapter 3. The threshold price is stored in the array CONV_AUTO_ENG_PR(isize,iyr).

6. Solves for the fraction of utilities that will select superconducting autotransformers as the area in the right-hand tail of the combined normal distribution constructed in Step 3 greater than the threshold energy price computed in Step 5. This area is calculated by calling subroutine CUM_NORMAL, after first converting to a normalized distribution with mean 0 and standard deviation 1 to facilitate computing the integral in the right-hand tail.

7. Adjusts the market penetration fraction calculated in Step 6 for superconducting autotransformers by multiplying the fraction of utilities that will select superconducting autotransformers by market penetration barriers that reflect "jitters," "tecnnical constraint," and "capital costs" described in Chapter 3.

8. Solves for the threshold energy price based on superconducting transformers such that any utility with combined load and no-load energy costs greater than this value will select superconducting autotransformers when installing new autotransformers. This equation is described in Chapter 3. The threshold price is stored in the array HTSC_AUTO_ENG_PR(isize,iyr).

9. Computes the number of new superconducting autotransformers that will be installed in the current year by multiplying the fraction of new superconducting autotransformers computed in Step 6 by the total number of new autotransformers computed in TOTAL NEW UNITS that will be installed in the current year. This result is stored in the array HTSC_AUTO_INSTALL(isize,iyr). The remainder of the new autotransformers installed in the current year, which are conventional, are stored in the array CONV_AUTO_INSTALL(isize,iyr).

10. Computes the cumulative number of superconducting and conventional autotransformers installed at the end of the current year and stores the results in the arrays HTSC_CUM_AUTO_INSTALL(isize,iyr) and CONV_CUM_AUTO_INSTALL(isize,iyr), respectively.

- (5.0-9.0) MKT_PENETR performs an analogous series of computational steps in the market penetration algorithm for step-up transformers:

1. Constructs three normal distributions for peaking, intermediate, and baseload energy costs using conventional step-up transformers based on the thre $\epsilon$ input parameters defining the mean values for peaking, intermediate, and baseload energy costs, respectively. For each 
distribution, the ratio of standard deviation to mean is assumed to be the same as that of the normal distribution for industrial energy prices fror U.S. utilities taken from Energy User News.

2. Constructs three normal distributions for peaking, intermediate, and baseload energy costs using superconducting step-up transformers based on the three input means in Step 1, the input mean for nonoperating energy costs, and the input fraction of total losses from nonoperating losses for superconducting step-up transformers. Using these input parameters, a weighted average of the means is computed for the three normal distributions. The ratio of standard deviation to mean is assumed to be the same as that of the normal distribution for industrial energy prices from U.S. utilities.

3. Solves for the threshold energy price for peaking, intermediate, and baseload operations based on conventional step-up transformers such that any utility with energy costs greater than this value will select superconducting step-up transformers for peaking, intermediate, and baseload operations, respectively. The equation is described in Chapter 3 . The threshold price is stored in the array CONV_STEPUP_ENG_PR(isize,iyr).

4. Solves for the threshold energy price for peaking, intermediate, and baseload operations based on superconducting step-up transformers such that any utility with combined nonoperating and operating energy costs greater than this value will select superconducting step-up transformers when installing new step-up transformers. This equation is described in Chapter 3. The threshold price is stored in the array HTSC_STEPUP_ENG_PR(isize,iyr).

5. Solves for the fraction of utilities that will select superconducting step-up transformers as the area in the right-hand tail of the normal distribution constructed in Step 1 greater than the threshold energy price computed in Step 3. This area is calculated by calling subroutine CUM_NORMAL.

6. Adjusts the market penetration fraction calculated in Step 4 for superconducting step-up transformers by multiplying the fraction of utilities that will select superconducting step-up transformers by the three market penetration barriers described in Chapter 3 .

7. Computes the number of new superconducting step-up transformers that will be installed in the current year in a similar manner to that of Step 9 for autotransformers. This result is stored in the array HTSC_STEPUP_INSTALL(isize,iyr). The remainder of the new step-up transformers installed in the current year, which are conventional, are stored in the array CONV_STEPUP_INSTALL(isize,iyr).

8. Computes the cumulative number of superconducting and conventional step-up transformers installed at the end of the current year and stores the results in the arrays HTSC CUM_STEPUP_INSTALL(isize,iyr) and CONV_CUM_STEPUT_INSTALL(isize, iyr), respectively.

- (5.0, 10.0) CUM_NORMAL calculates the cumulative standardized normal distribution function, i.e., the area under the standard normal curve (mean 0 and standard deviation 1 ) from 
minus infinity to $\mathbf{X}$. Because there is no closed-form solution, the integration is approximated using a fifth-order Taylor series expansion. The approximation was taken from Handbook of Mathematical Functions (Abramowitz and Stegun 1964, p. 932). CUM_NORMAL is called by both subroutines MKT_PENETR and BENEFITS to calculate the area in the right-hand tails of standardized normal distributions, which are evaluated as 1. - CUM_NORMAL(X).

- (9.5) UPDATE INVENT updates the inventory of conventional and superconducting autotransformers and the inventory of conventional and superconducting step-up transformers in each size and age group from the end of the previous year to the end of the current year. UPDATE INVENT is called each time step by the MAIN program from the first year of the simulation to the final year of market penetration. For conventional auto and step-up transformers, the inventory in the first age group ( 0 years, i.e., new units) is computed as the new conventional auto and step-up transformers installed at the end of the current year, respectively. For all other age groups (age $>1$ year), the inventory is computed by subtracting the failures and retirements occurring during the current year in the age group one year younger from the inventory of this age group at the end of the previous year. The updated auto and step-up inventories are stored in the three-dimensional arrays CONV_AUTO_INVENT (isize,iage_cell,iyr) and CONV_STEPUP_INVENT(isize,iage_cell, iyr), respectively.

Beginning with the first year of market penetration, UPDATE_INVENT analogously computes the inventory of superconducting auto and step-up transformers at the end of the current year using the existing inventory of superconducting transformers at the end of the previous year, the new superconducting transformers installed during the current year, and the failures and retirements of superconducting transformers occurring during the current year.

- (10.0) BENEFITS calculates the societal benefits of adopting superconducting transformers using two principal measures of performance. The first consists of 1) the annual energy savings (billions of kilowatt hours) in each size group from adopting superconducting auto and step-up transformers, 2) the total annual energy savings from both superconducting auto and step-up transformers summed over all size groups, and 3) the cumulative grand energy savings summed over all size groups and all years of the simulation, beginning with the first year of market penetration to the final year of the simulation.

The second measure of performance consists of the annual dollar savings (millions) from adopting superconducting auto and step-up transformers, including the capital savings, O\&M savings, energy loss savings converted to dollars, and the total of these three savings for each transformer type and size group, beginning with the first year of market penetration to the final year of the simulation. It also includes the total annual dollar savings from the surviving superconducting auto and step-up transformers in each size group discounted to dollars in the first year of the simulation and the cumulative discounted dollar savings from the surviving superconducting auto and step-up transformers in each size group summed over all years of the simulation.

- (10.0) BENEFITS is called once by the MAIN program after the iterative loop processing subroutines REPLACEMENTS, TOTAL_NEW_UNITS, MKT_PENETR, and UPDATE INVENT in Figure D.2 is completed from the first year of the simulation through the final year of market penetration. BENEFITS first calls subroutine SURVIVING_INVENT to update the inventory of surviving superconducting auto and step-up transformers in each size and 
age group from the end of the first year after the final year of market penetration to the final year of the simulation. During this timeframe, no new superconducting transformers are installed, but calculating the benefits to the nation past the final year of market penetration requires the inventory of surviving superconducting transformers in this timeframe. The results are stored in the arrays HTSC_AUTO_INVENT (isize,iage_cell,iyr) and HTSC_STEPUP INVENT (isize,iage_cell,iyr). BENEFITS then performs the following principal computational steps each year, beginning with the first year of market penetration to the final year of the simulation, to compute the annual energy savings:

1. Computes the number of superconducting auto and step-up transformers surviving in the current year in each size group, summed over all age groups. The results are stored in the arrays HTSC_AUTO_SURVIVE(isize,iyr) and HTSC_STEPUP_SURVIVE (isize,iyr), respectively.

2. Computes the annual energy savings (billions of kilowatt hours) in the current year in each size group from the surviving superconducting auto and step-up transformers.

3. Computes the annual energy savings in the current year summed over both surviving superconducting auto and step-up transformers and all size groups.

4. Computes the cumulative grand total of energy savings by summing the energy savings computed in Step 3 over all years.

- (10.0) BENEFITS next performs the following principal computational steps each year, beginning with the first year of market penetration to the final year of the simulation, to compute the annual dollar savings:

1. Calculates the average value of the utility energy prices greater than the threshold energy price based on conventional transformers for selecting superconducting transformers calculated in Step 5 for auto transformers and Step 3 for step-up transformers in subroutine MKT_PENETR. This average is computed in the current year for each size group as the sum of two integrals defined in Chapter 3. The first integral is approximated by calling subroutine CUM_NORMAL, after first converting to a standardized normal distribution with mean 0 and standard deviation 1 . The second integral has a closed-form solution. The results are stored in the arrays CONV_AUTO_ENG_PR_UPPER_AVE(isize,iyr) and CONV_STEPUP_ENG_PR_UPPER_ĀVE(sizē,ivr).

2. Calculates the average value of the utility energy prices greater than the threshold energy price based on superconducting transformers for selecting superconducting transformers calculated in Step 8 for auto transformers and Step 4 for step-up transformers in subroutine MKT_PENETR. The results are stored in the arrays HTSC AUTO_ENG_TR UPPER_AVE (isize,iyr) and HTSC_STEPUP_ENG_PR_UPPER_AVEE(isize,iyr), respectively. 
3. Calculates the change in capital costs from the superconducting auto and step-up transformers installed in the current year in each size group. The results are stored in the arrays AUTO_CAP_SA $\sqrt{\mathrm{k} I N G}$ (isize,iyr) and STEPUP_CAP_SAVING (isize,iyr), respectively.

4. Calculates the change in O\&M costs from the surviving superconducting auto and step-up transformers in the current year in each size group. The results are stored in the arrays AUTO_CAP_SAVING (isize,iyr) and STEPUP_OM_SAVING (isize,iyr), respectively.

5. Calculates the dollar value of the energy savings from the surviving superconducting auto and step-up transformers in the current year in each size group. The results are stored in the arrays AUTO_ENG_DOL_SAVING (isize,iyr) and STEPUP_ENG_DOL_SAVING (isize,iyr), respectively.

6. Calculates the dollar value of the total savings from the superconducting auto and step-up transformers in the current year in each size group by summing the savings calculated in Steps 3, 4, and 5. The results are stored in the arrays AUTO_TOT_SAVING (isize,iyr) and STEPUP_TOT_SAVING (isize,iyr), respectively.

7. Discounts the total savings calculated in Step 6 to dollars of the starting year of the simulation. The input discount rate used in the calculations is assumed to remain constant over the lifetime of the simulation. The results are stored in the arrays AUTO_TOT_DISC_SAVING (isize,iyr) and STEPUP_TOT_DISC_SAVING (isize,iyr), respectively.

8. Calculates the grand total savings from the surviving superconducting auto and step-up transformers in each size group by summing the savings calculated in Step 7 over all years. The results are expressed in dollars discounted to the starting year of the simulation and stored in the arrays AUTO_GRAND_TOT_DISC_SAVING (isize) and STEPUP_GRAND_TOT_DISC_SAVING (isize), respectively.

- (11.0) OUTPUT is called once at the end of the simulation by the MAIN program to write the results of the simulation to the DOS output file HTSC.PRN. This file is then copied by the DOS batch file HTSC.BAT together with the three input data files to the DOS output file HTSC.PRT for later disposition to the printer. OUTPUT calls subroutines PRT_MATRIX2D and PRT_MATRIX3D to print the many two- and three-dimensional matrices, respectively, involved in the simulation.

\section{D.3 Reference}

Abramowitz and Stegun. 1964. National Bureau of Standards Applied Mathematics Series 55. 


\section{Distribution}

No. of

Copies

Offsite

12 DOE/Office of Scientific and Technical Information

20

J. G. Daley CE-142

Office of Energy Management

U.S. Department of Energy

1000 Independence Avenue SW

Washington, DC 20585

R. Eaton CE-142

Office of Energy Management

U.S. Department of Energy

1000 Independence Avenue SW

Washington, DC 20585

M. E. Gunn, Jr.

EE-14

Office of Energy Management

U.S. Department of Energy

1000 Independence Avenue SW

Washington, DC 20585

A. DasGupta

U.S. Department of Energy

Chicago Operations Office

9800 South Cass Avenue

Argonne, IL 60439

R. Garabaldi

U.S. Department of Energy

Chicago Operations Office

9800 South Cass Avenue

Argonne, IL 60439

R. H. Aten

Office of Technology Policy and Studies

Room 4226

U.S. Department of Commerce

Washington, DC 20230
No. of

Copies

Offsite

B. C. Belanger

Advanced Technology Program

National Institute of Standards and Technology

Gaithersburg, MD 20899

G. Eyring

Office of Technology Assessment

600 Pennsylvania Avenue SE

Washington, DC 20510

J. Christian

Code RP

National Aeronautics and Space Administration

700 Independence Avenue SW

Washington, DC 20546

T. Bickel

Sandia National Laboratories

Division 6221

P.O. Box 5800

Albuquerque, NM 87185

S. L. Bogart

General Dynamics Space Division

P.O. Box 85990

Mail Zone 92-8260

San Diego, CA 91238

J. W. Bray

General Electric Research and Development

Building K1, Room IC25

P.O. Box 8

Schenectady, NY 12301 
No. of

Copies

Offsite

J. Carr

Westinghouse Electric Corporation

Science \& Technology Center

1310 Beulah Road

Pittsburgh, PA 15235

C. Carter

Electrical Systems Group

Tennessee Valley Authority

Missionary Ridge Place

2N46A Chattanooga Office Complex

Chattanooga, TN 37404

P. Chu

Texas Center for Superconductivity

University of Houston

Houston, TX 77204-5932

E. W. Collings

Battelle Columbus Laboratories

505 King Avenue

Columbus, OH 43201-2693

D. Connolly

Space Electronics Division, MS 54-1

NASA/Lewis Research Center

2100 Brookpark Road

Cleveland, OH 44135

J. Emerick

Superconductivity, Inc.

2114 Eagle Drive

Middleton, WI 53662

D. S. Ginley

National Renewable Energy

Laboratory

1617 Cole Boulevard

Golden, CO 80401
No. of

Copies

Offsite

R. Greene

Center for Superconductivity

College Park Campus

University of Maryland

College Park, MD 20742

D. U. Gubser

Materials Science \& Technology

Division

Naval Research Laboratory-Code 6300

4555 Overlook Avenue SW

Washington, DC 20375-5000

D. J. Hansen

Utah Power \& Light Company

168 N. 1950 W.

Salt Lake City, UT 84140

B. Hassenzahl

Lawrence Berkeley Laboratory

MS 2-400

Berkeley, CA 94720

R. Hawsey

Oak Ridge National Laboratory

P.O. Box 2008

Oak Ridge, TN 37831-6070

C. J. Heyne

Westinghouse Electric Corporation

Research \& Development Center

1310 Beulah Road

Pittsburgh, PA 15235

N. Hingorani

Electrical Systems Division

Electric Power Research Institute

P.O. Box 10412

Palo Alto, CA 94303 
No. of

Copies

Offsite
No. of

Copies

Offsite

\section{K. Holte}

Research and Development

Southern California Edison

P.O. Box 800

Rosemead, CA 91770

H. Kaufman

ESEERCO

28th Floor

1155 Avenue of the Americas

New York, NY 10036

J. C. King

Northwest Power Planning Council

851 S.W. 6th Avenue, Suite 1100

Portland, OR 97204

J. J. Iannucci

Distributed Utility Associates

627 Orion Way

Livermore, CA 94550

J. L. Kirtley, Jr.

Massachusetts Institute of Technology

Mailstop 10-093

77 Massachusetts Avenue

Cambridge, MA 02139

J. H. Lambert

Advanced Engineering

Pacific Power Company

920 SW 6th Avenue

Portland, OR 97204

D. C. Larbalestier

Engineering Research Building

University of Wisconsin

1500 Johnson Drive

Madison, WI 53706
D. H. Liebenberg

Office of Naval Research - Code 1112

800 North Quincy Street

Arlington, VA 22217-5000

J. Loncoski

Corporate Technical Assessment

Virginia Electric Power Company

5000 Dominion Boulevard

Glen Allen, VA 23060

R. W. Lighthipe

San Diego Gas and Electric $110 \mathrm{~W}$ "A" Street, Suite 4150

San Diego, CA 92112-4150

A. Malozemoff

American Superconductor

Corporation

149 Grove Street

Watertown, MA 02172

T. P. Mauldin

Pacific Gas and Electric Company 3400 Crow Canyon Road

San Ramon, CA 94583

B. W. McConnell

Oak Ridge National Laboratory

P.O. Box 2008, MS-6070

Oak Ridge, TN 37831-6070

R. McConnell

National Renewable Energy

Laboratory

1617 Cole Boulevard

Golden, CO 80401

G. H. Morgan

Brookhaven National Laboratory

Upton, NY 11973 
No. of

Copies

Offsite

C. C. Mosher

Department of Electrical and Computer Engineering

Washington State University

Pullman, WA 99164-2752

W. E. Myers

Bonneville Power Administration

P.O. Box 3621

Portland, OR 97208

D. Nordell

Northern States Power

414 Nicollet Mall

Minneapolis, MN 55401

K. D. Ott

CSAC

6th Floor

1050 Thomas Jefferson Street NW

Washington, DC 20007

A. D. Patton

Department of Electrical Engineering

Texas A\&M University

College Station, TX 77843

D. Peterson MS-K763

Superconducting Technology Program

Los Alamos National Laboratory

P.O. Box 1663

Los Alamos, NM 87545

R. Poeppel

Argonne National Laboratory

9700 South Cass Avenue

Argonne, IL 60439-4838

R. Schainker

Electric Power Research Institute

P.O. Box 10412

Palo Alto, CA 94304
No. of

Copies

Offsite

T. Schneider

Electric Power Research Institute

P.O. Box 10412

Palo Alto, CA 94304

S. M. Schoenung

W. J. Schafer Associates, Inc.

303 Lindberg Avenue

Livermore, CA 94550-9511

D. T. Shaw

New York State Institute on

Superconductivity

SUNY/Buffalo

330 Bonner

Amherst, NY 14260

D. M. Smyth

Materials Research Center

Building 32

Lehigh University

Bethlehem, PA 18015

J. Stekly

Field Effects, Inc.

6 Eastern Road

Acton, MA 10720

M. Superczynski

Code 2712

David Taylor Research Center

Annapolis, MD 21402-5067

R. S. Tamosaltis

E. I. du Pont de Nemours \& Co., Inc.

Experimental Station, Building 304

P.O. Box 80304

Wilmington, DE 19880-0304

\section{$y$}

2 
No. of

Copies

Offsite

B. C. Thomas

Puget Sound Power and Light

Company - OBC-09

P.O. Box 97034

Bellevue, WÀ 98009-9734

J. Vancoevering

Oak Ridge National Laboratory

P.O. Box 2008, MS-6070

Oak Ridge, TN 37831-6070

M. S. Walker

Intermagnetics General Corp.

P.O. Box 586

Charles Industrial Park

Guilderland, NY 12084

T. L. Weaver

Western Area Power Administration

P.O. Box 3402

Golden, CO 80401

B. Wenning

TU Electric

40 N. Olive, LB. 81

Dallas, TX 75201

T. A. Williams

National Renewable Energy

Laboratory

1617 Cole Boulevard

Golden, CO 80401

F. Young

Electrical Systems Division

Electric Power Research Institute

P.O. Box 10412

Palo Alto, CA 94303

G. J. Yurek

American Superconductor Corporation

149 Grove Street

Watertown, MA 02172-2828
No. of

Copies

Foreign

F. Chu

Research Division - KR151

Ontario Hydro

800 Kipling Avenue

Toronto, Ontario M8Z 5S4

CANADA

Onsite

DOE Richland Operations Office

D. D. Green

Westinghouse Hanford Company

S. B. Merrick

15

\section{Pacinc Northwest Laboratory}

J. W. Currie

J. E. Dagle

J. G. De Steese (2)

J. A. Dirks

H. D. Huber

R. W. Reilly

S. A. Smith

I. W. White

Publishing Coordination

Technical Report Files (5) 

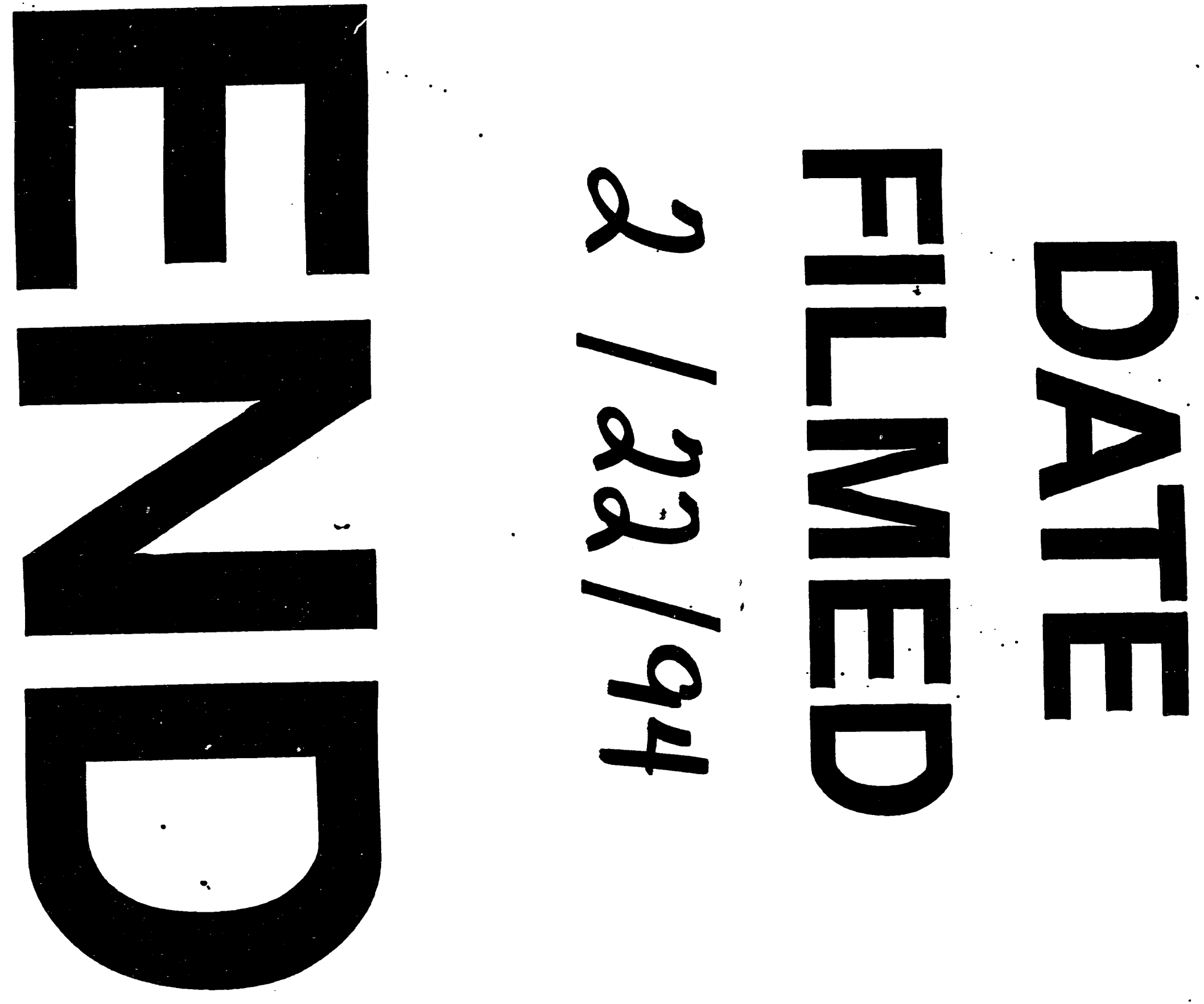
